Departamento de Ingeniería Energética

Escuela Técnica Superior de Ingenieros Industriales

Universidad Politécnica de Madrid
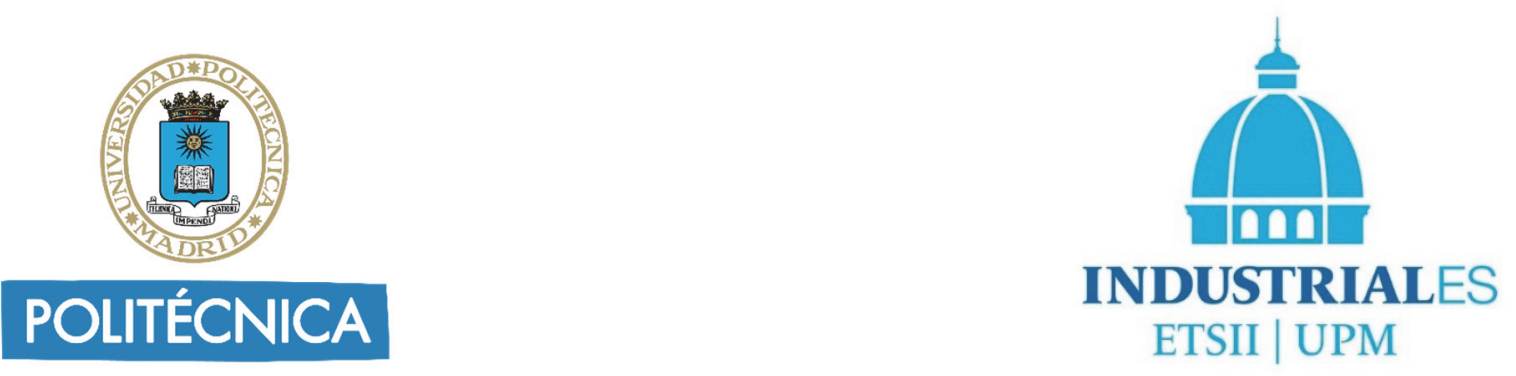

\title{
A New CONCEPT IN THERMAL ENGINEERING OPTIMIZATION: \\ the Pericritical Cycle With \\ Multi-Heating AND ITS APPlication to Concentrating Solar Power
}

Luis Francisco González Portillo

Ingeniero Industrial

Director:

Javier Muñoz Antón

Dr. Ingeniero Industrial 
Para Ignacio, sobrino, ahijado y futuro inventor 
"Learn what is to be taken seriously and laugh at the rest."

- Herman Hesse 


\begin{abstract}
This work performs a detailed analysis of the integration of pericritical cycles (cycles with the compressor working in the surroundings of the critical point) in Concentrating Solar Power plants. A coherent integration between these elements results in a plant composed of a novel thermodynamic cycle layout, called multi-heating cycle, and a new type of solar field. The specific features of multi-heating cycles fit perfectly with the characteristics of concentrating solar energy, which results in a system with high potential to reduce the costs of electricity production in plants of this type of energy.
\end{abstract}

The multi-heating cycle is presented as a solution to reduce the high irreversibilities of regeneration in pericritical cycles. While the heat in simple regenerative cycles is supplied by means of a single heat source, in multiple heating cycles part of that heat is replaced by others supplied at lower temperature by additional heat sources. The exergy efficiency of multiheating cycles is greater than in simple regenerative cycles due to the lower exergetic cost of additional heat sources.

Simple regenerative pericritical cycles and multi-heating cycles are studied by means of a comprehensive and systematic thermodynamic analysis. Reduced thermo-physical properties define these cycles with the purpose of generalizing its characterization setting aside the fluid. The analysis of the pericritical region shows the special relevance of the so-called discontinuity line, which separates the thermodynamic diagram into two regions: liquid and gaseous. The cycle performance is characterized according to the compressor position in the thermodynamic diagram with respect to the discontinuity line and to the cycle conditions.

The greater exergy efficiency of multi-heating cycles with respect to simple cycles comes at the expense of bigger intermediate heat exchangers. The volume of the heat exchangers is calculated using printed circuit heat exchangers. The use of $\mathrm{CO}_{2}$ as working fluid shows that wet-cooled cycles achieve higher exergy efficiencies with smaller heat exchanger volumes than dry-cooled cycles.

The integration of multi-heating cycles in Concentrating Solar Power plants requires the design of a new type of solar field to accommodate the solar radiation impinging on the receiver to the multi-heating requirements. This new solar field has a configuration similar to the configuration of a solar tower, but with the receiver and the heliostats divided into two sections. Each section meets different requirements in concentration ratio, fluid temperature, and absorbed heat flux. This new solar field achieves greater efficiencies than standard solar towers, but with lower thermal requirements. A techno-economic analysis shows that a system with multi-heating cycle and the new solar field not only achieves high performance, but also a great potential to reduce the costs of CSP plants. 


\section{RESUMEN}

Este trabajo lleva a cabo un análisis detallado del potencial que tiene la integración de ciclos pericríticos (ciclos con el compresor trabajando en las proximidades del punto crítico) en plantas de energía solar de concentración. Una coherente integración entre estos elementos da como resultado una planta compuesta por un novedoso ciclo termodinámico, bautizado como ciclo de calentamiento múltiple, y un nuevo tipo de campo solar. Las características específicas del ciclo de calentamiento múltiple encajan perfectamente con las características de la energía solar de concentración, lo que da como resultado un sistema con un alto potencial para reducir los costes de producción de electricidad en plantas de este tipo de energía.

El ciclo de calentamiento múltiple se presenta como solución para reducir las altas irreversibilidades de la regeneración en ciclos pericríticos. Mientras que el calor en ciclos regenerativos simples se suministra por medio de una única fuente de calor, en ciclos de calentamiento múltiple parte de ese calor se sustituye por otros suministrados a menor temperatura por fuentes de calor adicionales. La eficiencia exergética de los ciclos de calentamiento múltiple es mayor que la de los ciclos regenerativos simples debido al menor coste exergético de sus fuentes adicionales de calor.

Los ciclos pericríticos regenerativos simples y los ciclos de calentamiento múltiple se estudian mediante un detallado y sistemático análisis termodinámico. La definición de estos ciclos se realiza mediante propiedades termo-físicas reducidas con el propósito de generalizar la caracterización dejando de lado el fluido. El análisis de la región pericrítica muestra la especial relevancia de la llamada línea de discontinuidad, que separa el diagrama termodinámico en dos regiones: la líquida y la gaseosa. El rendimiento del ciclo es caracterizado en función de la posición en el diagrama termodinámico del compresor con respecto a la línea de discontinuidad y a las condiciones del ciclo.

La mayor eficiencia exergética de los ciclos de calentamiento múltiple con respecto a los ciclos simples se produce a costa de un mayor tamaño de los intercambiadores de calor necesarios durante la regeneración. El volumen de los intercambiadores de calor se calcula utilizando intercambiadores de calor de circuito impreso. El uso de $\mathrm{CO}_{2}$ como fluido de trabajo muestra que los ciclos de enfriamiento húmedo alcanzan mayor eficiencia exergética con intercambiadores de calor más pequeños que los ciclos de enfriamiento seco.

La integración de los ciclos de calentamiento múltiple en centrales de energía solar de concentración requiere el diseño de un nuevo tipo de campo solar para acomodar la radiación solar incidente en el receptor a los requisitos del calentamiento múltiple. Este nuevo campo solar tiene una configuración similar a la configuración de una torre solar, pero con el 
receptor y los heliostatos divididos en dos secciones, cada una con diferentes requisitos en relación de concentración, temperatura del fluido y flujo de calor absorbido. Este nuevo campo solar alcanza una mayor eficiencia que una torre solar estándar, pero con menores requisitos térmicos. Un análisis tecno-económico muestra que un sistema con ciclo de calentamiento múltiple y el nuevo campo solar no solo tiene un alto rendimiento, sino que también posee un gran potencial para reducir los costes de las plantas de energía solar de concentración. 


\section{AGRADECIMIENTOS (SPANISH)}

Siempre he dicho que dar las gracias es gratis, y qué mejor momento que éste para aplicarse el cuento y tirar de "gratistud" (broma hecha, check). Así pues, me gustaría daros las gracias a todos los que hayáis aportado algo en mi vida, así, en general, que como digo, es gratis. Pero en especial me gustaría agradecer el apoyo de todos los que me habéis acompañado durante esta última etapa de mi vida de tesinando.

Mis primeros agradecimientos van dirigidos a aquellos que me abrieron la puerta del mundo académico y que, desde entonces, han sido mis guías sapienciales. José $\mathrm{M}^{\mathrm{a}}$ (boss), Javier, gracias por darme la oportunidad de comenzar este camino y confiar en mí.

Una puerta no se abre si no se llama a ella, y nunca habría podido llamar sin el apoyo de los más cercanos. Elisa, familia, mil gracias por apoyar a un loco a que dejara un trabajo que llenaba sus bolsillos por un trabajo que le llena de felicidad.

Muchos más sois los que me habéis acompañado durante los últimos años, y mi cariño hacia vosotros es inversamente proporcional a mi memoria, así que perdonadme si no me acuerdo en este momento de alguno. Mis agradecimientos:

A los compis de la cátedra, que me acogisteis con los brazos abiertos (como para no, por otro lado) y no dejáis de enseñarme algo nuevo cada día.

A los jóvenes compis de la cátedra vecina, que son unos liantes (especialmente los días antes de mis ponencias en congreso).

A mis amigos del bar. Álvaros, Balbás, gracias, sin vosotros habría terminado la tesis mucho antes.

A los del cole, por ese amor incondicional que solo otorgan los años de amistad. Juan, Joely, desde los tres años juntos. Vamos ...! (Rellenad vosotros el hueco).

A los de la uni, por seguir pensando que estoy loco por volver a la ETSII, pero quererme pese a todo.

Pa' los de Collao, por querer ganar al Risk.

To the Delft crew, my time there was awesome. I guess that I even learned something.

A los que no ligo a ningún grupo, pero con los que sigo manteniendo una gran amistad: Adrián “el mágico", Marta "gemelitos”, Luis “el majo” y Elena “la maja?”.

A todos, gracias. 


\section{SCIENTIFIC OUTCOMES}

During the development of this work, several research outcomes have been published: two articles, one patent and many conference proceedings. The list below shows the most significant outcomes (articles under review are not included):

\section{Articles}

González-Portillo, L.F., Muñoz-Antón, J. and Martínez-Val, J.M. (2017) 'An analytical optimization of thermal energy storage for electricity cost reduction in solar thermal electric plants', Applied Energy, 185, pp. 531-546.

González-Portillo, L.F., Muñoz-Antón, J. and Martínez-Val, J.M. (2019) 'Thermodynamic mapping of power cycles working around the critical point', Energy Conversion and Management, 192, pp. 359-373.

\section{Patents}

Martínez-Val, J.M., Muñoz-Antón, J., González-Portillo, L.F., Amengual Matas, R. (2019). 'Proceso cíclico termodinámico sin condensación del fluido con prescripciones acotadas sobre sus puntos de mínima y máxima entalpía y dispositivo para su realización', ES2652522B2.

\section{Conferences}

González-Portillo, L.F., Muñoz-Antón, J. and Martínez-Val, J.M. (2017), ‘Supercritical CO2 in a concentrating solar power plant with thermal energy storage'. Industrial Research Meeting '17, Madrid, ISBN 978-84-16397-58-7

González-Portillo, L.F., Muñoz-Antón, J. and Martínez-Val, J.M. (2017), 'Comparing the cost of storage in photovoltaics and concentrating solar power: batteries vs thermal energy storages'. 10th National Congress Engineering of Thermodynamics, Lleida.

González-Portillo, L.F., Muñoz-Antón, J. and Martínez-Val, J.M. (2017), 'Optimizing the configuration of concentrating solar power plants to reduce thermal energy cost'. 10th National Congress Engineering of Thermodynamics, Lleida.

González-Portillo, L.F. (2018), 'Solar thermal new engine: Coherent integration of solar tower and supercritical fluids'. II Edition Symposium: your tesis in 4 minutes, Madrid.

González-Portillo, L.F., Muñoz-Antón, J. and Martínez-Val, J.M. (2018), ‘A Different Way to Approach Enthalpy Loss in Supercritical Regenerative Closed Brayton Cycles: the Solar Heater'. SolarPACES 2018, Casablanca. 
Otero R., G.J., González-Portillo, L.F. and Pecnik, R. (2019), 'Turbulence modelling for supercritical CO2 flows in a vertical tube'. 3rd European Conference on Supercritical CO2 Power Systems, Paris.

González-Portillo, L.F., Muñoz-Antón, J. and Martínez-Val, J.M. (2019), 'Exergetic analysis of multi-heating cycles'. European Conference on Renewable Energy Systems (ECRES), Madrid. 


\section{CONTENTS}

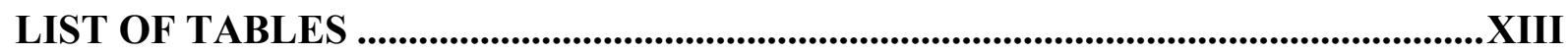

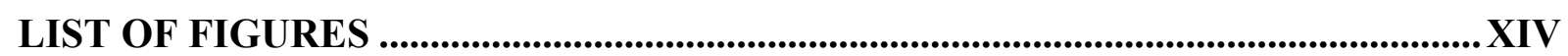

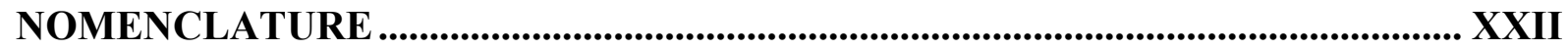

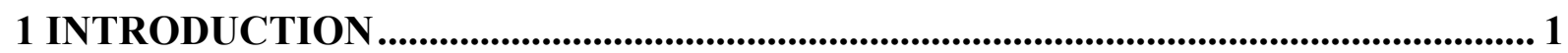

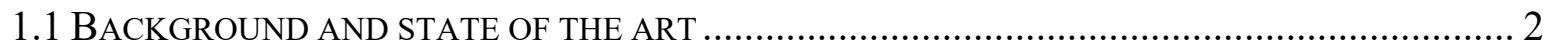

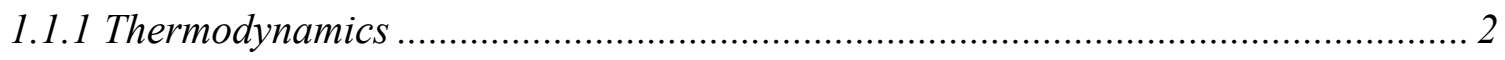

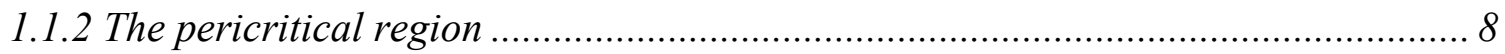

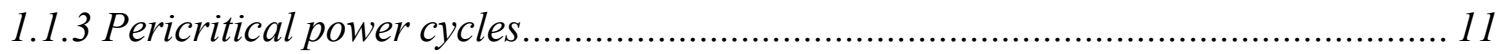

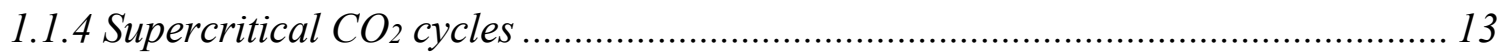

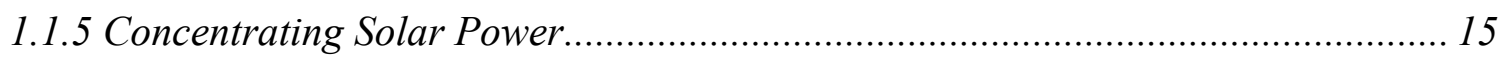

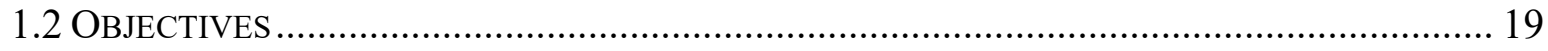

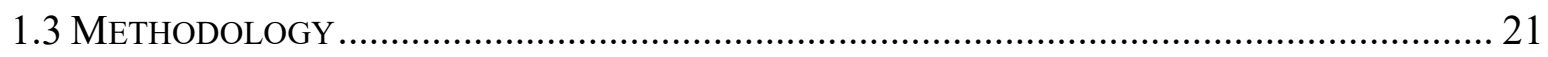

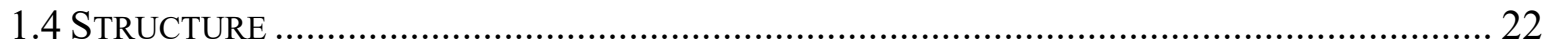

2 THERMODYNAMIC MAPPING OF PERICRITICAL CYCLES .............................. 24

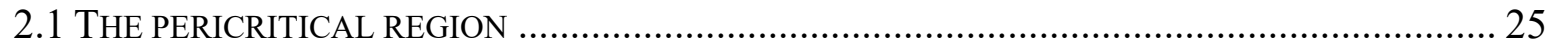

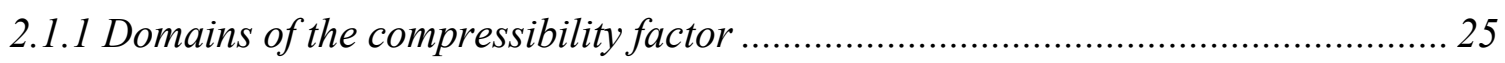

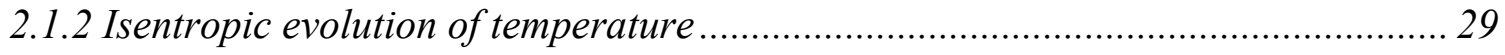

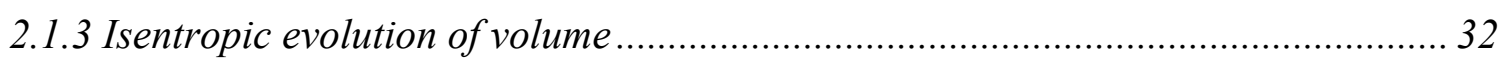

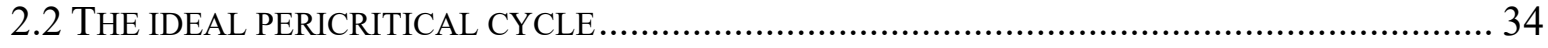

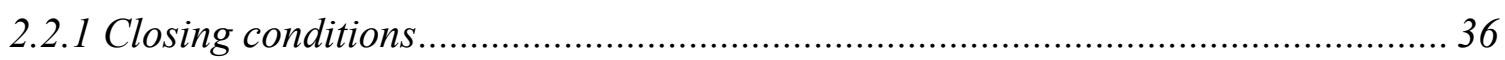

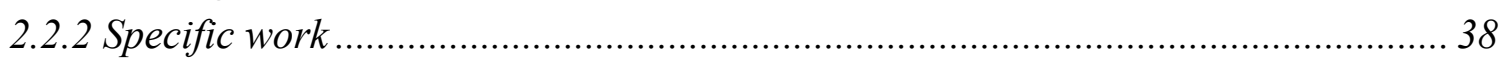

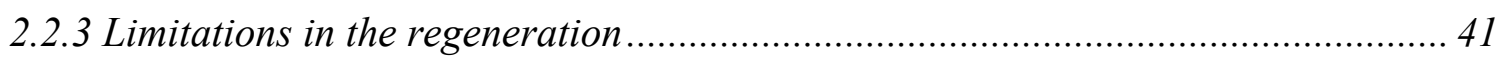

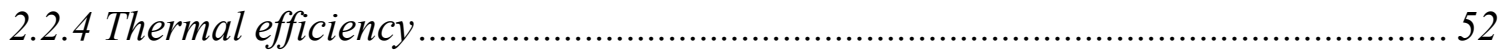

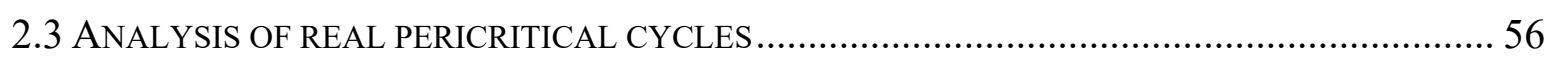

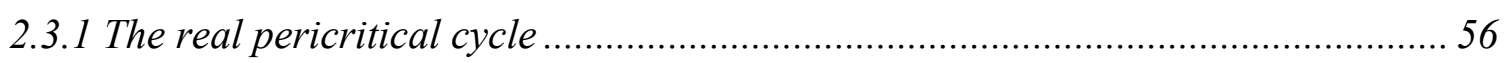

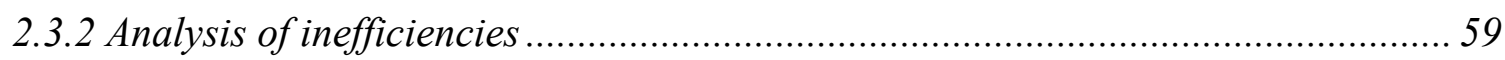

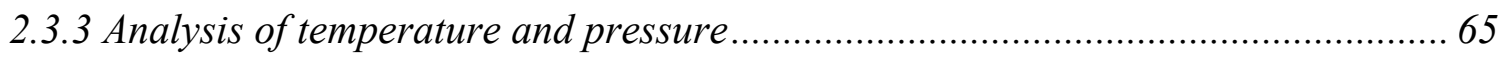

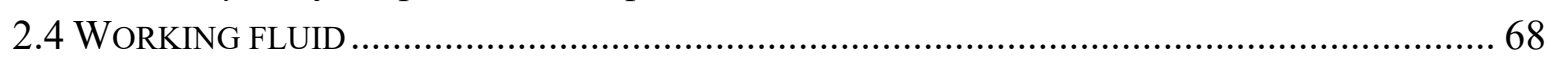

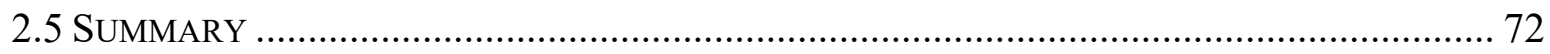

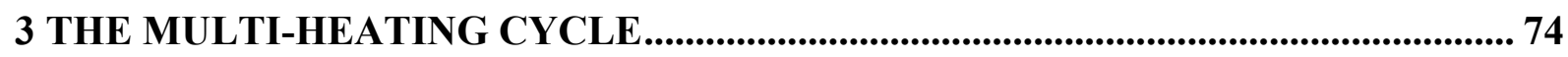

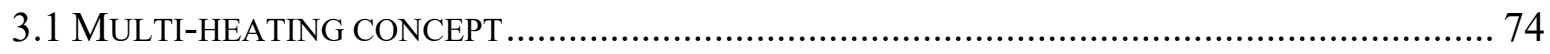

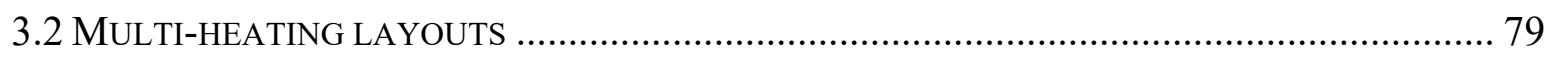

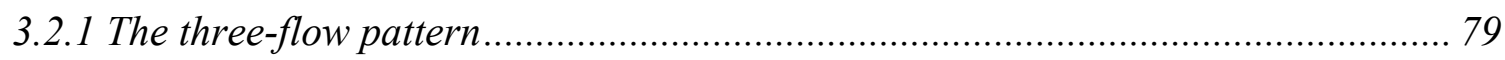

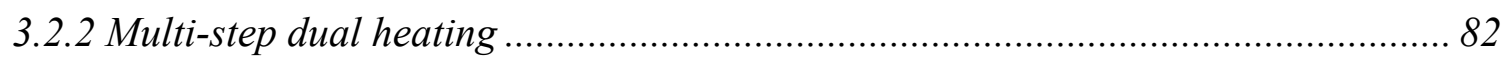

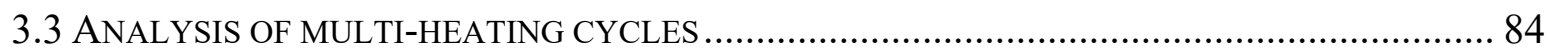

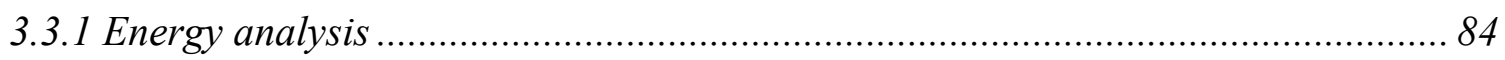

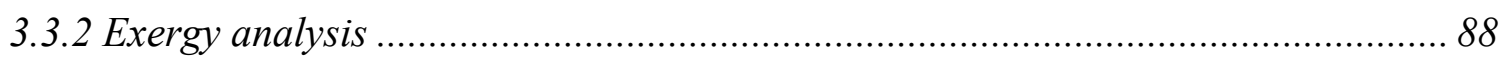

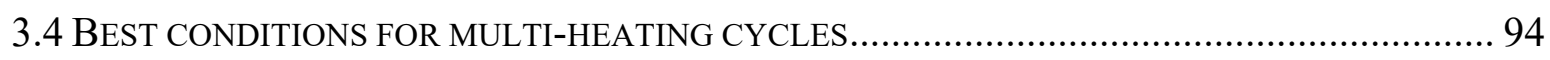

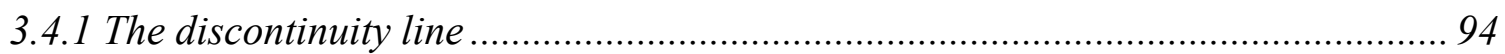




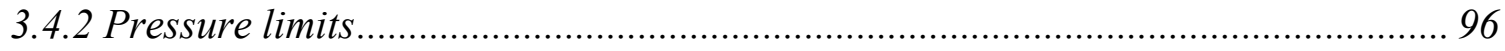

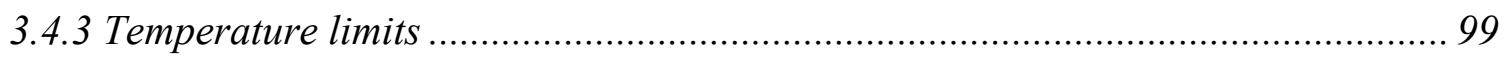

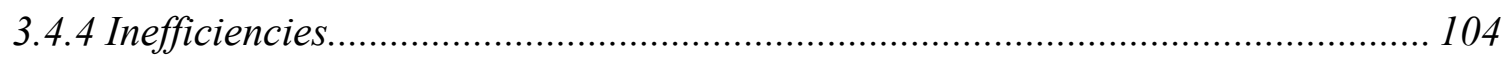

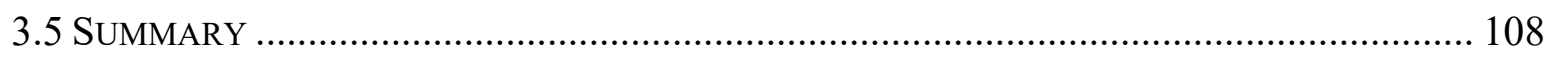

4 HEAT EXCHANGERS IN MULTI-HEATING CYCLES ........................................... 110

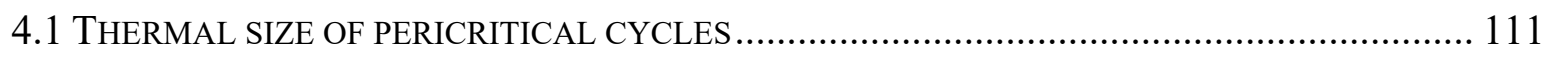

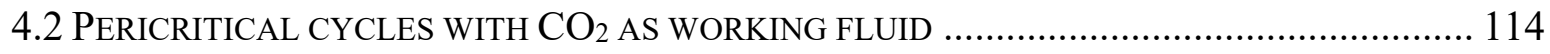

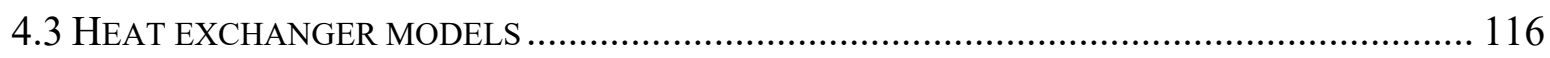

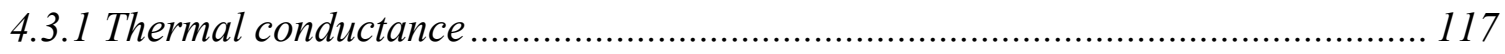

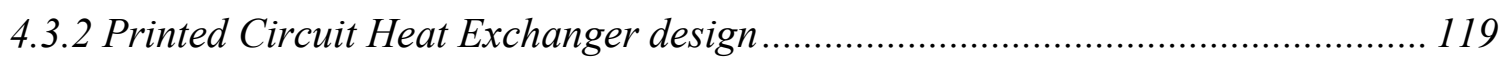

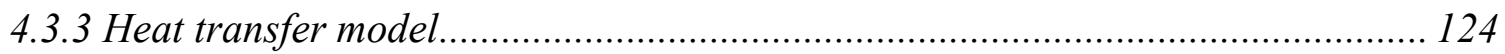

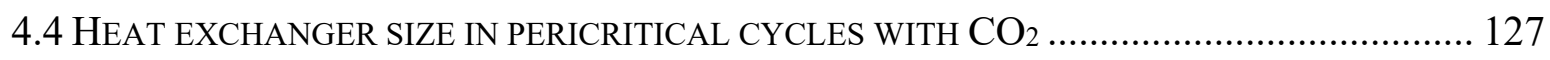

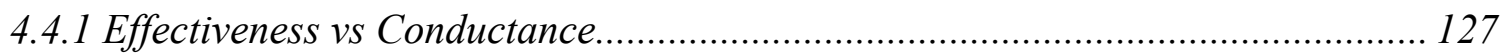

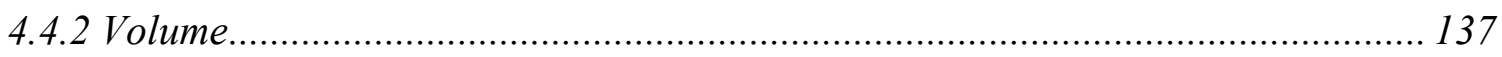

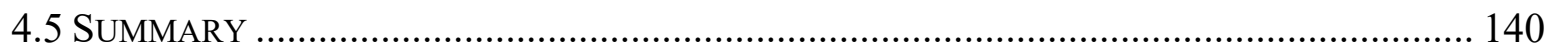

5 MULTI-HEATING CYCLES IN CONCENTRATING SOLAR POWER

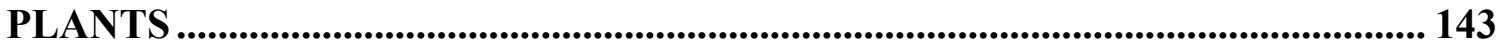

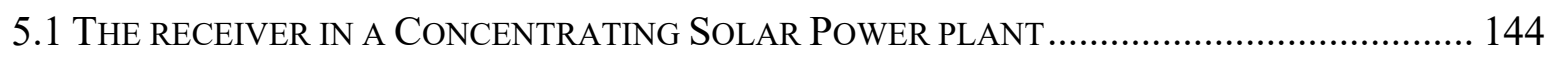

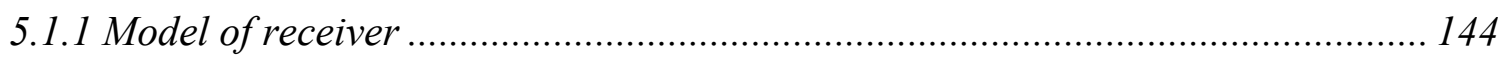

5.1.2 Benchmark against System Advisor Model ......................................................... 148

5.1.3 Power block needs....................................................................................... 150

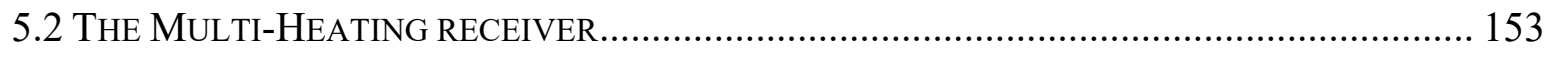

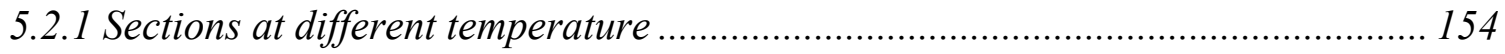

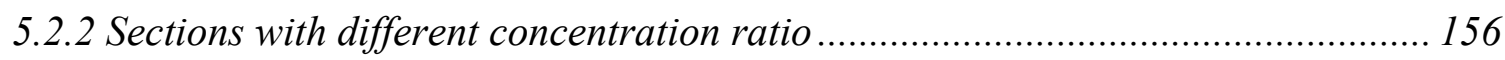

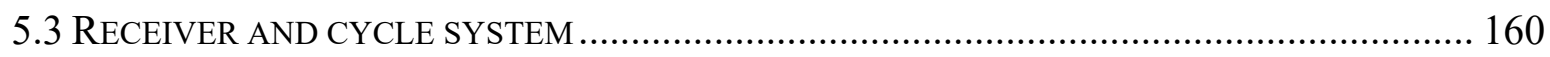

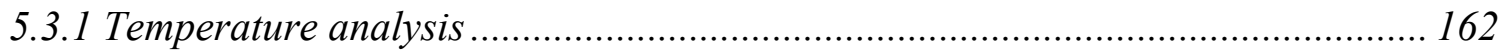

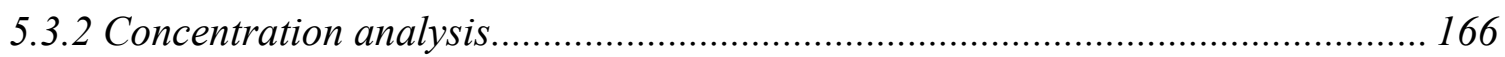

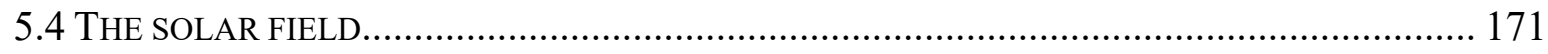

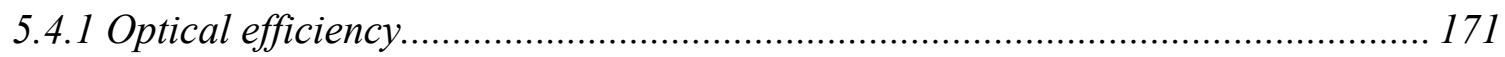

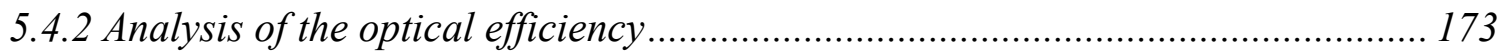

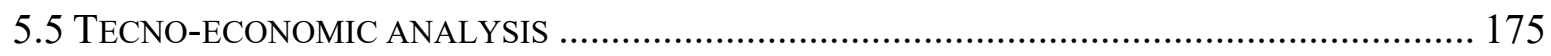

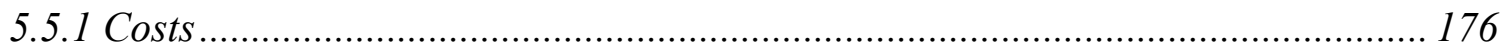

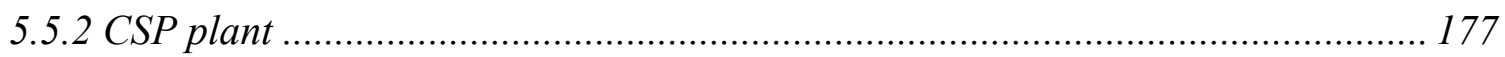

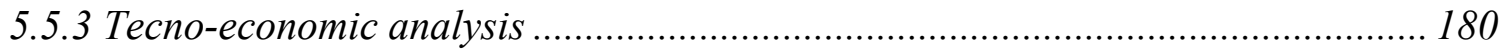

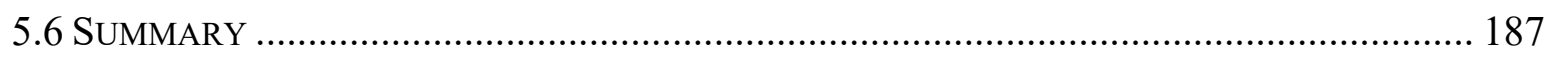

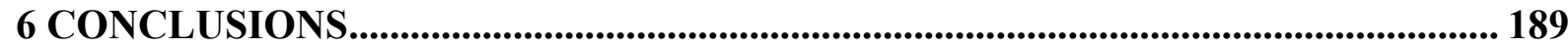

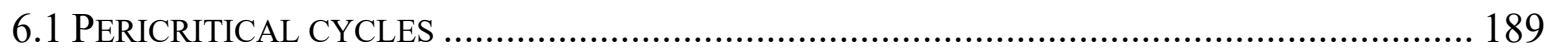

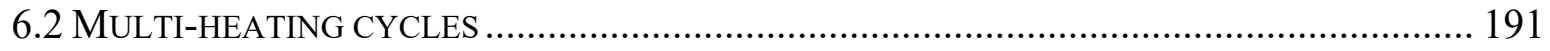

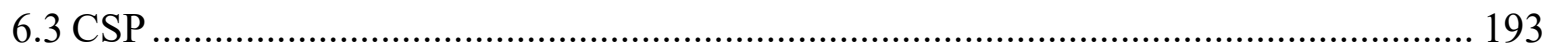

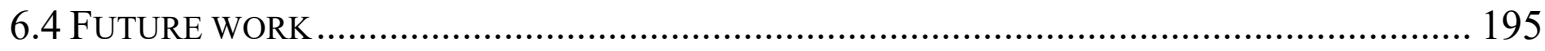

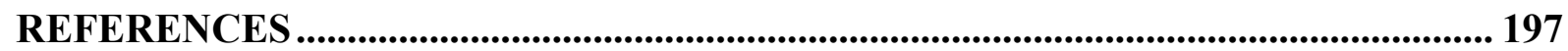


APPENDICES 204

APPENDIX A - FLUID CANDIDATES FOR PERICRITICAL CYCLES . 205

APPENDIX B - CORRELATIONS USED IN THE HEAT EXCHANGER DESIGN 206

APPENDIX C - SPECIFICATIONS SHEET OF PRINTED CIRCUIT

HEAT EXCHANGER . 209 


\section{LIST OF TABLES}

Table 3.1. Parameters used to analyze the regeneration in a pericritical cycle........................ 76

Table 3.2. Parameters used to analyze multi-heating pericritical cycles ................................ 87

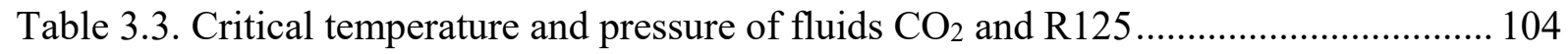

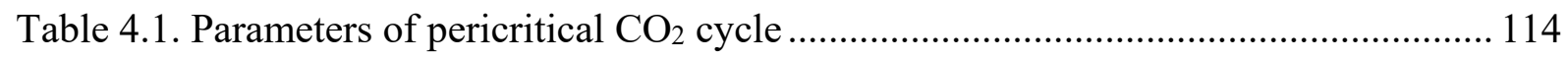

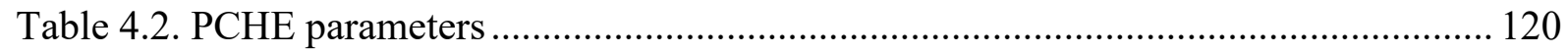

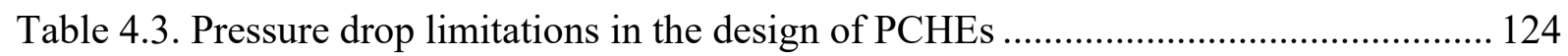

Table 4.4. Size of the components depending on the cycle type ......................................... 141

Table 5.1. Input parameters used for the analysis of a solar thermal receiver ....................... 146

Table 5.2. Parameters of $\mathrm{CO}_{2}$ cycle in a CSP plant ........................................................ 178

Table 5.3. Parameters of precooler, primary heat exchanger and additional haet

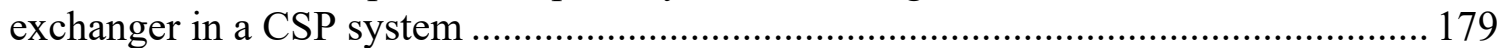

Table 5.4. Parameters of solar field in a CSP system ....................................................... 180

Table 5.5. System configuration of the CSP systems depending on its optimization............. 183

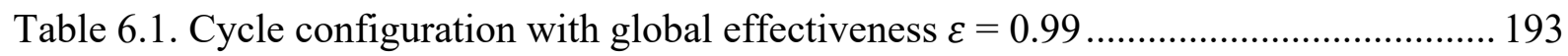




\section{LIST OF FIGURES}

Figure 1.1. Ideal closed cycle in diagrams P-h (left) and T-s (tight). Temperatures are equal at compressor outlet and turbine outlet.

Figure 1.2. Regenerative closed cycle in P-h diagram.

Figure 1.3. P-h diagrams of $\mathrm{CO}_{2}$ with isothermal and isentropic lines (top), and isolines of compressibility factor, $z$, and isobaric expansion coefficient, $\alpha$, in terms of $1 / T$ (bottom)

Figure 1.4. Compressibility factor, $z$, of $\mathrm{CO}_{2}$ versus density, 100/v, for different values of pressure and temperature (expressed as a function of the critical pressure, $P_{c r}$, and critical temperature, $T_{c r}$, respectively)

Figure 1.5. Pressure-temperature (P-T) diagram divided into four quadrants with the critical point as common point (Banuti et al., 2017)

Figure 1.6. Pressure-temperature (P-T) diagram divided into four quadrants with the critical point as common point and the Widom line (dashed), dividing the supercritical quadrant into a liquid-like (subscript LL) and a gas-like (subscript GL) region (Banuti et al., 2017).

Figure 1.7. Density, $\rho$, and isobaric specific heat capacity, $c_{p}$, of $\mathrm{CO}_{2}$ at subcritical, critical and supercritical pressures as a function of temperature, $T$.

Figure 1.8. Thermal efficiencies of power conversion systems and applications. (Ahn et al., 2015)

Figure 1.9. Comparison of turbine sizes for steam, helium and $\mathrm{CO}_{2}$ (Dostal, 2004)

Figure 1.10. Scheme of a CSP plant with solar tower (NREL, 2017).

Figure 1.11. LCOE (in $\mathrm{c} € / \mathrm{kwh}$ ) as a function of storage size (in hours) with PV+battery and CSP+TES (González-Portillo, Muñoz-Antón and MartínezVal, 2017a)

Figure 1.12. Receiver efficiency, $\eta_{r e c}$, Carnot efficiency, $\eta_{C}$, and system efficiency, $\eta_{\text {syst }}$, as a function of maximum system temperature, $T_{h o t}$, with the subscript indicating the concentration ratio $C=10, C=100$ and $C=1000$......

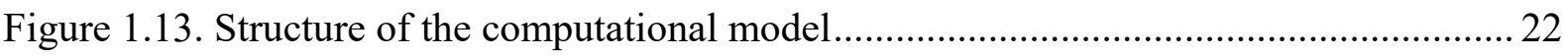

Figure 2.1. Fluid "states" in a pressure-temperature diagram.............................................. 24

Figure 2.2. P-h diagram of $\mathrm{CO}_{2}$ around the critical point showing isolines of compressibility factor, $z$, and logarithmic factor of isobaric expansion, $f_{P}$.

Figure 2.3. Fluid states separated by the discontinuity line in a pressuretemperature diagram.

Figure 2.4. P-h diagram of $\mathrm{CO}_{2}$ around the critical point showing isolines of compressibility factor, $z$, and logarithmic factor of isothermal expansion, $f_{T}$.

Figure 2.5. P-h diagram of $\mathrm{CO}_{2}$ around the critical point showing isolines of compressibility factor, $z$, and isentropic evolution of temperature coefficient, $g$.

Figure 2.6. Pericritical cycle working partially above the critical pressure in diagrams P-h (left) and T-s (right) ..... 
Figure 2.7. Pericritical cycle working above the critical pressure in diagrams P-h (left) and T-s (right)......

Figure 2.8. Pericritical cycles with compressor inlet reduced temperature $T_{c, i n, r}=$ 0.95 (left) and $T_{c, i n, r}=1.05$ (right)

Figure 2.9. Parameter $w_{c} / w_{t}$ and coefficient $m_{c}$ as a function of the pressure ratio, $r$, in a pericritical cycle with compressor inlet temperatures $T_{c, i n, r}=0.95$ and $T_{c, i n, r}=1.05$.

Figure 2.10. Specific heat, $c_{p}$, of $\mathrm{CO}_{2}$ as a function of the temperature, $T$, for different pressures below and above the critical pressure $\left(P_{c r}\right)$

Figure 2.11. Schematic diagram of a generic pericritical regenerative cycle limited by the points corresponding to the compressor inlet (c,in), compressor outlet (c,out), turbine inlet (t,in) and turbine outlet $(t, o u t)$. Some linearized isothermal lines $T_{x}$ are shown inside the cycle.

Figure 2.12. Values of the derivative $(d h / d P)_{T}$ and logarithmic factor of isobaric expansion in the low-pressure and high-pressure sides, $f_{P, L P}$ and $f_{P, H P}$, respectively, as a function of the temperature, $T$, in a cycle working between the critical pressure and a pressure $P_{r}=3$

Figure 2.13. Schematic diagram of a pericritical regenerative cycle with the isothermal line with minimum $(d h / d P)_{T}$ outside the cycle.

Figure 2.14. Enthalpy balance at differential scale in part of the cycle with two isothermal lines and two isobars.

Figure 2.15. Dimensionless specific heat, $c_{p} / R$, and temperatures, $T_{r}$, in the highand low-pressure sides of regeneration of a cycle with the minimum $(d h / d P)_{T}$

(a) inside the cycle and (b) outside the cycle

Figure 2.16. Schematic diagram of a pericritical regenerative cycle with the minimum $(d h / d P)_{T}$ inside, showing the additional enthalpy increments needed in primary heat and precooler, $\Delta h_{a, p h}$ and $\Delta h_{a, p c}$, due to the specific heat mismatch of the regeneration phase.

Figure 2.17. Schematic diagram of a pericritical regenerative cycle with the minimum $(d h / d P)_{T}$ outside the cycle, showing the additional enthalpy increment needed in primary heat, $\Delta h_{a, p h}$, due to the specific heat mismatch of the regeneration phase.

Figure 2.18. Maximum enthalpy variation of an isothermal line during regeneration, $\Delta h_{r g}$, as a function of the compressor inlet temperature, $T_{c, i n, r}$, for different pressure ratios....

Figure 2.19. Maximum enthalpy variation of an isothermal line during regeneration, $\Delta h_{r g}$, and specific turbine work, $w_{t}$, as a function of the pressure ratio, $r$, for cycles with low-side pressure $P_{L, r}=1$ and different compressor inlet temperatures $T_{c, i n, r}$

Figure 2.20. Dimensionless additional enthalpy, $\Delta h_{r g} / w_{t}$, as a function of the pressure ratio, $r$, in a pericritical cycle with low-side pressure $P_{L, r}=1$ and compressor inlet temperatures $T_{c, i n, r}=0.95$ and $T_{c, i n, r}=1.05$.

Figure 2.21. Dimensionless compression work, $w_{c} / w_{t}$, dimensionless additional enthalpy, $\Delta h_{r g} / w_{t}$, and thermal efficiency, $\eta_{t h}$, as a function of the pressure ratio, $r$, in a pericritical cycle with low-side pressure $P_{L, r}=1$ 
Figure 2.22. P-h diagram of pericritical cycles working at high-side pressure $P_{H, r}$ $=3$ and different low-side pressures and compressor inlet temperatures

Figure 2.23. Dimensionless compression work, $w_{c} / w_{t}$, dimensionless additional enthalpy, $\Delta h_{r g} / w_{t}$, and thermal efficiency, $\eta_{t h}$, as a function of the pressure ratio, $r$, in a pericritical cycle with high-pressure side $P_{H, r}=3$

Figure 2.24. Diagram of a simple regenerative pericritical cycle (inspired by Dyreby (2014))

Figure 2.25. Example of simple regenerative pericritical cycle in diagrams $\mathrm{P}-\mathrm{h}$ (left) and T-s (right). The numbers mark the beginning and end of each phase.

Figure 2.26. Parameters $\Delta h_{r g} / w_{t}$ and $w_{c} / w_{t}$, and thermal efficiency $\eta_{\text {th }}$ as a function of the pressure ratio, $r$, in pericritical cycles with high-pressure side $P_{H, r}=3$ and different compressor inlet temperatures and inefficiencies.

Figure 2.27. Thermal efficiency $\eta_{\text {th }}$ as a function of the pressure ratio, $r$, in pericritical cycles with high IHX effectiveness

Figure 2.28. Optimum pressure ratio, $r_{\text {opt }}$, and thermal efficiency, $\eta_{t h}$, as a function of the IHX effectiveness, $\varepsilon$, for different values of turbomachine efficiencies, $\eta_{c}$

Figure 2.29. Thermal efficiency, $\eta_{t h}$, as a function of the low-side pressure, $P_{L, r}$, for different high-side pressures, $P_{H, r}$, and compressor inlet temperatures, $T_{c, i n, r}$

Figure 2.30. Thermal efficiency, $\eta_{t h}$, and optimum low-side pressure, $P_{L, o p t, r}$, as a function of high-pressure side, $P_{H, r}$, for different compressor inlet temperatures, $T_{c, i n, r}$

Figure 2.31. Classification of fluids as a function of its critical temperature, $T_{c r}$, and critical pressure, $P_{c r}$

Figure 2.32. Discontinuity line of $\mathrm{CO}_{2}$ and $\mathrm{R} 125$ in P-h diagram

Figure 2.33. Thermal efficiency, $\eta_{t h}$, of pericritical cycles with $\mathrm{CO}_{2}$ and $\mathrm{R} 125$ as working fluid as a function of the high-side pressure, $P_{H, r}$, and compressor inlet temperature, $T_{c, i n,}$

Figure 3.1. Scheme of a pericritical cycle with infinite additional heats supplied during the regeneration in diagrams P-h (left) and T-s (right)....

Figure 3.2. Additional heats, $q_{a h, i}$, needed along the regeneration of a pericritical cycle in order to balance the intermediate heat exchanger.

Figure 3.3. Temperatures of the high-pressure side, $T_{H P}$, and low-pressure side, $T_{H P}$, along the regenerator of a pericritical cycle with infinite additional heats supplied during the regeneration (in red), and without additional heats (in black).

Figure 3.4. Additional heat, $q_{a h}$, supplied during the regeneration by means of infinite heats supplied up to a temperature, $T_{a h}$, in order to reduce the regeneration heat losses $h_{a}$ and $h_{r g}$, in a cycle that needs a total heat, $q_{\text {total }}$.

Figure 3.5. Diagram of a regenerative pericritical cycle with the three-flow pattern layout (diagram design inspired by Dyreby (2014)) 
Figure 3.6. Possible embodiments of the three-flow heat exchanger. The fluid supplying the additional heat, $a h$, is in the outer part and the low-pressure, $L P$, and high-pressure, $H P$, fluids in the inner part.

Figure 3.7. Scheme of a pericritical cycle with the three-flow pattern layout in diagrams P-h (left) and T-s (right)

Figure 3.8. Temperatures of the high-pressure side, $T_{H P}$, and low-pressure side, $T_{H P}$, along the regenerator of a pericritical cycle with three-flow pattern layout (in red), and without additional heats (in black).

Figure 3.9. Diagram of a regenerative pericritical cycle with the step dual heating layout (diagram design inspired by Dyreby (2014))

Figure 3.10. Scheme of a pericritical cycle with the step dual heating layout in diagrams P-h (left) and T-s (right)

Figure 3.11. Temperatures of the high-pressure side, $T_{H P}$, and low-pressure side, $T_{H P}$, along the regenerator of a pericritical cycle with the step dual heating layout (in red), and without additional heats (in black).

Figure 3.12. Heats, $q$, of a multi-heating cycle and its associated Carnot efficiencies, $\eta_{c}$, as a function of the additional-heat temperature, $T_{a h}$. Primary heat, $p h$, additional heat, $a h$, and total heat, total, are represented.

Figure 3.13. Exergy inputs of the primary heat, $e_{i n, p h}$, additional heat, $e_{i n, a h}$, and total heat, $e_{i n, t o t a l}$, and cycle exergetic efficiency, $\eta_{e x}$, as a function of the additional-heat temperature, $T_{a h}$.

Figure 3.14. Exergy losses, $i$, of the components of a multi-heating cycle as a function of the additional-heat temperature, $T_{a h}$. These components are: compressor $(c)$, turbine $(t)$, precooler $(p c)$, primary heat exchanger $(p h)$, additional heat exchanger $(a h)$, low temperature regenerator (LTR), high temperature regenerator $(H T R)$.

Figure 3.15. Exergy efficiency, $\eta_{e x}$, and temperature of the second additional heat, $T_{a h 2}$, of a multi-heating cycle with two additional heats as a function of the temperature of the first additional heat $T_{a h 1}$

Figure 3.16. Exergetic efficiency, $\eta_{e x}$, of a multi-heating cycle as a function of the number of additional heats

Figure 3.17. Exergetic efficiency, $\eta_{e x}$, of a multi-heating cycle with different numbers of additional heats, $N_{a h}$, as a function of the low-pressure side, $P_{L, r}$. The high pressure is set to $P_{H, r}=3$.

Figure 3.18. Parameters $w_{c} / w_{t}$ and $\Delta h_{r g} / w_{t}$ of a pericritical cycle as a function of the low-pressure side, $P_{L, r}$. The high pressure is set to $P_{H, r}=3$.

Figure 3.19. Optimum low-side pressure, $P_{L, r}$, as a function of the number of additional heats for different high-side pressures, $P_{H, r}$.

Figure 3.20. Exergy efficiency, $\eta_{e x}$, as a function of the number of additional heats for different high-side pressures, $P_{H, r}$

Figure 3.21. Exergy efficiency, $\eta_{e x}$, and optimum low-side pressure, $P_{L, r}$, of a multi-heating cycle with $N_{a h}$ additional heats as a function of the compressor inlet temperature, $T_{c, i n, r}$ 
Figure 3.22. Exergy efficiency, $\eta_{e x}$, as a function of high-side pressure, $P_{H, r}$, and compressor inlet temperature, $T_{c, i n, r}$, in a simple cycle (top) and a multiheating cycle (bottom)

Figure 3.23. Relative increase in exergy efficiency, $\Delta \eta_{e x}$, of supplying an additional heat to a simple cycle as a function of high-pressure side, $P_{H, r}$, and compressor inlet temperature, $T_{c, i n, r}$

Figure 3.24. Relative increase in exergy efficiency, $\Delta \eta_{e x}$, of supplying an additional heat to a simple cycle with R125 as a function of high-pressure side, $P_{H, r}$, and compressor inlet temperature, $T_{c, i n, r}$

Figure 3.25. Exergy efficiency, $\eta_{e x}$, as a function of the compressor inlet efficiency, $\eta_{c}$, and $\mathrm{HX}$ effectiveness, $\varepsilon$, in cycles with compressor inlet temperature $T_{c, i n, r}=0.95$ (left figures) and $T_{c, i n, r}=1.05$ (right figures), and no additional heats (top figures) and one additional heat (down figures)

Figure 3.26. Optimum pressure ratio, $r$, as a function of the compressor inlet efficiency, $\eta_{c}$, and HX effectiveness, $\varepsilon$, in cycles with compressor inlet temperature $T_{c, i n, r}=0.95$ (left figures) and $T_{c, i n, r}=1.05$ (right figures), and no additional heats (top figures) and one additional heat (down figures)

Figure 3.27. Relative increase of exergy efficiency, $\Delta \eta_{e x}$, of supplying one additional as a function of the compressor inlet efficiency, $\eta_{c}$, and $\mathrm{HX}$ effectiveness, $\varepsilon$, in cycles with compressor inlet temperature (a) $T_{c, i n, r}=0.95$ and (b) $T_{c, i n, r}=1.05$

Figure 4.1. Specific enthalpy variation, $\Delta h$, in each phase of a multi-heating cycle as a function of the reduced compressor inlet temperature, $T_{c, i n, r}$

Figure 4.2. Mass flow rate, $m$, and pressure ratio, $r$, of a cycle with nominal electric power of $50 \mathrm{MW}$ depending on the compressor inlet temperature, $T_{c, i n, r}$

Figure 4.3. Thermal power of the heat exchange parts of a simple $\left(N_{a h}=0\right)$ and multi-heating cycle $\left(N_{a h}=1\right)$ as a function of the compressor inlet temperature, $T_{c, i n, r}$

Figure 4.4. Heat exchanger discretization

Figure 4.5. (a) Reduced temperatures, $T_{r}$, and (b) conductances, $U A$, of $N_{H X}=40$ sub-heat exchangers distributed along the regeneration of a simple $\left(N_{a h}=0\right)$ and a multi-heating cycle with $N_{a h}=1$.

Figure 4.6. Scheme of a PCHE cross-section (Chen et al., 2013).

Figure 4.7. Volume and pressure drop of a PCHE with conductance $U A=4050$ $\mathrm{kW} / \mathrm{K}$ as a function of the total number of channels

Figure 4.8. Heat transfer coefficient, $h t c$, as a function of the bulk fluid temperature, $T_{\text {fluid }}$....

Figure 4.9. Exergy efficiency, $\eta_{e x}$, pressure ratio, $r$, and conductance, $U A$, as a function of the IHX effectiveness, $\varepsilon$, in wet- and dry-cooled cycles with one heat supply (simple) and two heat supplies (multi-heating)

Figure 4.10. Exergy efficiency, $\eta_{e x}$, of multi-heating wet-cooled cycle with different global conductance values, $U A$, as a function of the LTR fraction, $x_{L T R}$ 
Figure 4.11. Heat exchangers effectiveness, $\varepsilon$, of multi-heating wet-cooled cycles with different global conductance values, $U A$, as a function of the LTR fraction, $x_{L T R}$

Figure 4.12. Exergy efficiency, $\eta_{e x}$, and pressure ratio, $r$, as a function of the global conductance, $U A$. Wet-cooled cycles to the left and dry-cooled cycles to the right.

Figure 4.13. Heat exchanger effectiveness, $\varepsilon$, as a function of global conductance, $U A$, for simple and multi-heating cycles (wet-cooled to the left and drycooled to the right)

Figure 4.14. Exergy efficiency, $\eta_{e x}$, of multi-heating dry-cooled cycles as a function of the global conductance value, $U A$, obtained with three different methods: conductance, $U A$, effectiveness, $\varepsilon$, and a mix of both.

Figure 4.15. Volume of regenerator and precooler as a function of the global regenerator conductance, $U A$, in simple $(\mathrm{S})$ and multi-heating $(\mathrm{MH})$ cycles in the cases of wet-cooling and dry-cooling.....

Figure 4.16. Exergy efficiency, $\eta_{e x}$, of simple and multi-heating cycles as a function of the total heat exchangers volume. The dashed line separates wetcooled (to the left) and dry-cooled (to right) cycles.

Figure 5.1. Receiver efficiency, $\eta_{r e c}$, as a function of the optical concentration ratio, $C_{o p t}$, for different HTF temperatures, $T_{H T F}$.

Figure 5.2. Receiver efficiency, $\eta_{r e c}$, Carnot efficiency, $\eta_{C}$, and system efficiency, $\eta_{\text {syst }}$, as a function of the turbine inlet temperature, $T_{t, i n}$, for optical concentration ratios $C_{o p t}=50, C_{o p t}=200$ and $C_{o p t}=600$.

Figure 5.3. Receiver area, $A_{r e c}$, as a function of the optical concentration, $C_{o p t}$, for different temperatures

Figure 5.4. Temperatures of $\mathrm{CO}_{2}, \mathrm{HTF}$ and receiver surface as a function of the thermal power $Q C O 2$ required by a simple cycle

Figure 5.5. Thermal power in the receiver resulting from the energy balance in each step, Qstepi, as a function of the thermal power QCO2 required by a simple cycle.

Figure 5.6. Scheme of solar field with (a) Solar Tower and (b) Multi-Heating Solar Tower

Figure 5.7. Thermal power, $Q$, and receiver efficiency, $\eta_{r e c}$, of a Multi-Heating Solar Tower (MHST) and its two sections primary heat (ph) and additional heat ( $\mathrm{ah}$ ) as a function of the additional-heat temperature, $T_{a h}$

Figure 5.8. Receiver efficiency, $\eta_{r e c}$, of standard Solar Tower (ST) and MultiHeating Solar Tower (MHST) with two sections, primary heat (ph) and additional heat (ah), as a function of the turbine inlet temperature, $T_{t, i n}$

Figure 5.9. Receiver efficiency, $\eta_{r e c}$, of standard Solar Tower (ST) and MultiHeating Solar Tower (MHST) with two sections, primary heat (ph) and additional heat (ah), as a function of the optical concentration ratio, $C_{\text {opt }}$

Figure 5.10. Maximum fluid and surface temperatures, $T$, in the sections of a Multi-Heating Solar Tower as a function of the optical concentration ratio, Copt 
Figure 5.11. Receiver efficiency, $\eta_{r e c}$, of MHSTv2 and its two sections, primary heat (ph) and additional heat (ah), as a function of the concentration ratio for the additional-heat section, $C_{\text {opt }, \text { ah }}$, keeping constant the concentration ratio in the primary-heat section at $C_{o p t, p h}=600$

Figure 5.12. CSP plant with standard Solar Tower and simple regenerative cycle

Figure 5.13. CSP plant with Multi-Heating Solar Tower and multi-heating cycle.

Figure 5.14. Efficiencies of thermodynamic cycles $\eta_{\text {th }}$ and receivers $\eta_{\text {rec }}$ as a function of the turbine inlet temperature, $T_{t, i n}$. Cycles: simple (SC) and multiheating (MHC). Receivers: Solar Tower (ST), Multi-Heating Solar Towers version 1 and version 2 (MHSTv1 and MHSTv2).

Figure 5.15. Efficiencies of systems $\mathrm{SC}+\mathrm{ST}$ (simple cycle coupled to Solar Tower) and MHC+MHSTv1 and MHC+MHSTv2 (Multi-Heating cycle coupled to Multi-Heating Solar Tower versions 1 and 2)....

Figure 5.16. Optimum pressure ratio, $r_{\text {opt }}$, and system efficiency, $\eta_{s y s t}$, as a function of the turbine inlet temperature, $T_{t, i n}$, for systems $\mathrm{SC}+\mathrm{ST}$ (Simple cycle coupled to Solar Tower) and MHC+MHST (Multi-Heating cycle coupled to Multi-Heating Solar Tower) under wet- and dry-cooled conditions

Figure 5.17. Relative increase in system efficiency, $\Delta \eta_{\text {syst }}$, of multi-heating with respect to simple cycles as a function of the turbine inlet temperature, $T_{t, \text { in }}$

Figure 5.18. System efficiency, $\eta_{\text {syst }}$, as a function of the optical concentration ratio, $C_{o p t}$, for systems SC+ST (Simple cycle coupled to Solar Tower) and MHC+MHST (Multi-Heating cycle coupled to Multi-Heating Solar Tower) under wet- and dry-cooled conditions

Figure 5.19. Relative increase in system efficiency, $\Delta \eta_{\text {syst }}$, of multi-heating with respect to simple cycles as a function of the optical concentration ratio, $C_{\text {opt }}$.

Figure 5.20. System efficiency, $\eta_{s y s t}$, as a function of the additional-heat concentration ratio, $C_{o p t, a h}$, for systems $\mathrm{SC}+\mathrm{ST}$ (Simple cycle coupled to Solar Tower) and MHC+MHSTv2 (Multi-Heating cycle coupled to MultiHeating Solar Tower v2) under wet- and dry-cooled conditions....

Figure 5.21. Receiver surface, Arec, as a function of the additional-heat concentration ratio, $C_{o p t, a h}$.......

Figure 5.22. Optical efficiency of heliostats in a system with Solar Tower obtained with the software solarPILOT (Wagner and Wendelin, 2018)......

Figure 5.23. Efficiency of a CSP plant, $\eta_{C S P}$, as a function of the additional-heat concentration ratio, $C_{\text {opt,ah }}$. The subscript of the systems MHC+MHSTv2 stands for the optical efficiency of the concentration ratio in the additionalheat section.

Figure 5.24. Heliostats surface, Ahel, as a function of the additional-heat concentration ratio, $C_{\text {opt,ah }}$. The subscript of the systems MHC+MHSTv2 stands for the optical efficiency of the concentration ratio in the additionalheat section.

Figure 5.25. Cost of the power block in $\$ / \mathrm{kW}_{\mathrm{e}}$ depending on the type of cycle (simple or multi-heating) and the costs of the additional-heat section (determined by $\varphi$ ) 
Figure 5.26. Cost of the solar field in $\$ / \mathrm{kW}_{\mathrm{e}}$ depending on the type of receiver (solar tower, ST, and multi-heating solar tower, MHST) and the costs of the additional-heat section (determined by $\varphi$ ).

Figure 5.27. Cost of the CSP plant in $\$ / \mathrm{kW}_{\mathrm{e}}$ when the system efficiency is maximized.

Figure 5.28. Cost of the CSP plant in $\$ / \mathrm{kW}_{\mathrm{e}}$ when the system efficiency is maximized (eff) and when the system cost is minimized (cost)

Figure 5.29. Optimum pressure ratio, $r_{o p t}$, and additional-heat tempearture, $T_{a h}$, as a function of the parameter $\varphi$ accounting for the cost of the additional-heat section.

Figure 5.30. System cost in $\$ / \mathrm{kW}_{\mathrm{e}}$ as a function of the parameter $\varphi$ accounting for the costs of the additional-heat section

Figure 6.1. Cost comparison between a CSP plant with simple cycle and solar tower and a CSP plant with multi-heating cycle and multi-heating solar tower. 


\section{NOMENCLATURE}

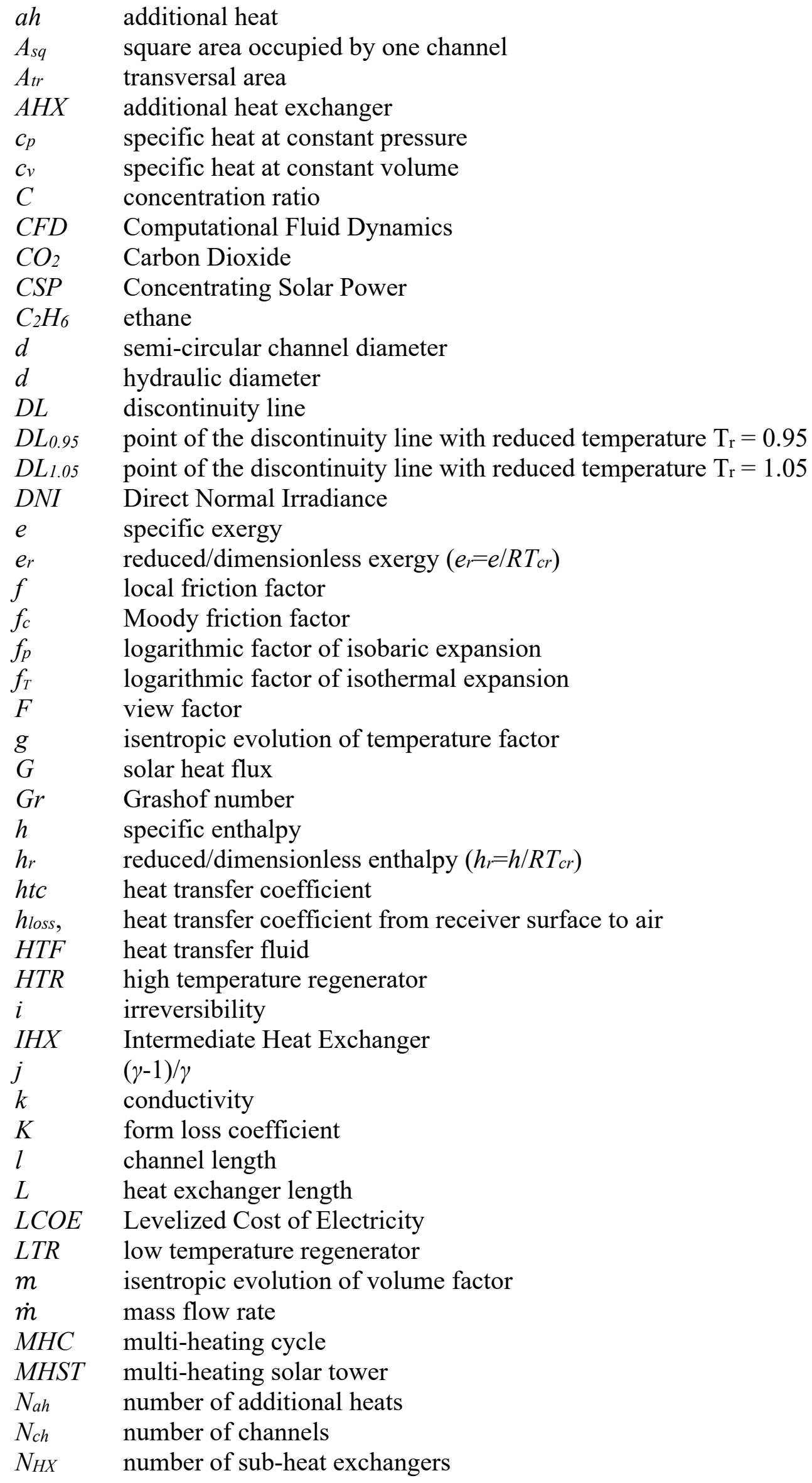




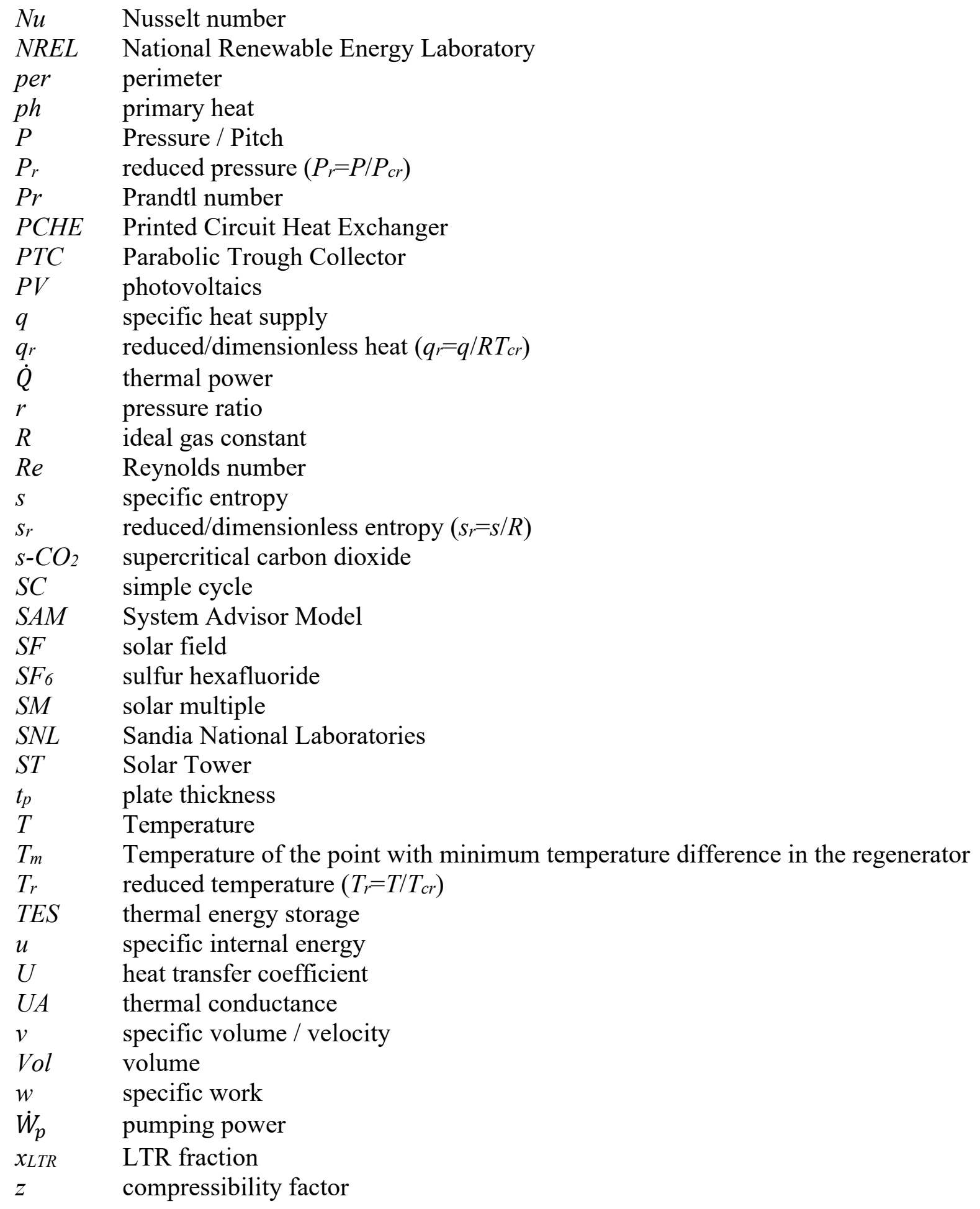

\section{Greek letters}

$\alpha$ dilatation coefficient at constant pressure / absorptivity

$\gamma \quad$ adiabatic coefficient or heat capacity ratio

$\varepsilon \quad$ effectiveness

$\varepsilon_{r} \quad$ emissivity

$\eta \quad$ efficiency

$\rho$ density

$\sigma \quad$ Stefan-Boltzmann constant

$\tau \quad$ Carnot factor $\left(\tau=\mathrm{T}_{\text {hot }} / \mathrm{T}_{\text {cold }}\right)$

$\varphi \quad$ ratio between additional-heat cost and primary-heat cost 
$\chi \quad$ pressure loss

$\Delta h_{a} \quad$ additional enthalpy increment or regeneration heat loss

$\Delta h_{r g} \quad$ enthalpy loss due to real gas conditions

$\Delta T_{l m} \quad$ logarithmic mean temperature difference

\section{Subscripts}

$\begin{array}{ll}a & \text { additional } \\ a h & \text { additional heat } \\ a m b & \text { ambient } \\ c & \text { compressor } \\ c r & \text { critical } \\ c v & \text { convection } \\ C & \text { Carnot } \\ \text { ex } & \text { exergy } \\ \text { geo } & \text { geometrical } \\ G L & \text { gas-like } \\ h e l & \text { heliostats } \\ H & \text { high (referred to pressure P) } \\ H P & \text { high pressure } \\ i g & \text { ideal gas } \\ \text { in } & \text { inlet/input } \\ \text { lm } & \text { logarithmic mean } \\ L & \text { low (referred to pressure P) } \\ L L & \text { liquid-like } \\ L P & \text { low pressure } \\ m a x & \text { maximum } \\ \text { opt } & \text { optimum / optical } \\ \text { out } & \text { outlet } \\ p c & \text { precooler } \\ \text { ph } & \text { primary heat } \\ P & \text { constant pressure } \\ r & \text { reduced } \\ r a d & \text { radiation } \\ r e c & \text { receiver } \\ r e f & \text { reference } \\ r e g & \text { regeneration } \\ r g & \text { real gas } \\ s & \text { constant entropy } \\ \text { syst } & \text { system } \\ t & \text { turbine } \\ t h & \text { thermal } \\ T & \text { Constant temperature } \\ v & \text { constant volume } \\ & \end{array}$




\section{INTRODUCTION}

Thermal power plants are power stations in which thermal energy is converted to electricity. The source of this thermal energy may be different, but all these plants need a power cycle to convert the thermal energy to mechanical energy, and an alternator to convert the mechanical energy to electricity. An example of thermal power plant is the case of Concentrating Solar Power (CSP) plants, which supply the thermal energy by means of solar radiation. The advantage of CSP with respect to other thermal sources such as fossil fuels or nuclear is that it uses a renewable energy source: the Sun.

In the field of renewable energy generation, CSP plants stand out due to its good storage capacity. While other type of renewable energies store energy by means of complex and expensive systems such as batteries, CSP plants may integrate an inherent, simple and cheap thermal storage. Nowadays, CSP plants are the only renewable energy (excluding hydropower) that can generate electricity during 24 hours in a row at a reasonable price. Nonetheless, the cost of CSP plants is still high, and the future of this technology will depend on the capability to become cheaper.

The solar field is the most expensive part of a CSP plant. Nevertheless, the power cycle performance is the one determining the amount of thermal power needed, which in CSP plants is translated to area of solar field. Therefore, solar field and power cycle have both the capacity of reducing the cost of CSP plants, and therefore they must be analyzed together.

There are many investigations about CSP systems with the purpose of reducing its cost. Most of them focus on how to increase the power cycle performance as much as possible and then integrate it with the solar field, or the opposite, how to increase the solar field performance as 
much as possible and then integrate it with the power cycle. This work presents an analysis where both parts are equally optimized by means of a coherent match between thermodynamics and heat management. The purpose of this match is to enhance the thermal coherence in order to find the way towards lower electricity costs in CSP plants.

The coherent design between thermodynamics and heat management will be found through a multidisciplinary pathway along the sections of this document. The foundations of this pathway are found in thermodynamics. A strong thermodynamic basis will help in the attainment of an efficient thermal power system. The thermodynamic analysis will focus in the surroundings of the critical point. The characteristic thermophysical properties of fluids in this region have already shown a promising future for several applications, in especial for CSP systems.

A simple thermodynamic analysis can obtain the highest cycle performance, but a thermodynamic analysis integrating the CSP basics will obtain the highest system performance. Heat management will be fundamental to integrate the CSP basics in the thermodynamic analysis, but also to complete the parts where thermodynamics cannot reach. The outcome of this analysis will be a CSP plant composed of an innovative thermodynamic cycle layout and a new type of solar field. The coherent integration of thermodynamics and heat management will result in a CSP plant with higher performance and potential lower costs.

\subsection{Background and state of the art}

This work presents a pathway that starts with thermodynamics focused on the region of the thermodynamic diagram surrounding the critical point and ends with an application to real Concentrating Solar Power plants. The first step of this pathway is to state the basis of thermodynamics and some specific features of the region around the critical point. After the introduction of these important phenomena the focus will shift to present the current status of power cycles working around the critical point and its application to CSP plants.

\subsubsection{Thermodynamics}

Thermodynamics is a branch of physics that succeeded even before being formulated. The best example is the Industrial Revolution, in which the steam engines powered factories and pushed trains before the laws of thermodynamics were properly defined. In fact, the 
development of thermodynamics began in 1824, when Carnot established the second law of thermodynamics due to the desire of increasing the efficiency of steam engines.

Some decades later, the steam turbine substituted the steam engine in the field of electricity generation, and nowadays it is responsible for producing most of the world's electricity in thermal power plants. While the steam turbine substituted the steam engine for large powers, the internal combustion engines did it for medium and small powers. Internal combustion engines were the key in the inventions of automobiles and airplanes, and both industries enjoyed many decades without important changes in the engines. However, since the first modern gas turbine was built in 1939, internal combustion engines started to disappear in aircrafts, remaining only for very small powers. The gas turbine became the dominant propulsion motor for aircrafts in a few years due to its flexibility, reaction speed following acceleration requirements and power density.

Gas turbines, also referred to as Brayton cycles, were not used in electricity generation at the beginning because steam turbines, also referred to as steam Rankine cycles, had higher efficiencies. This is still true for ordinary ranges of temperature in current heat sources. Nevertheless, advancements in materials and components for aircraft engines were projected to the electricity industry, which involved several novelties in electricity generation followed by a great increase in efficiency thanks to combined cycles (Brayton + Rankine).

Regardless of the type of cycle, thermodynamics establishes the relation between the variation of enthalpy (h), entropy (s) and pressure (P) by means of Equation 1.1.

$$
d h=T d s+v d P
$$

The ideal closed cycle is made of two isentropic lines $(d s=0)$, which correspond to compression and expansion, and two isobars $(d P=0)$, which correspond to heating and cooling. The four phases (compression, heating, expansion and cooling) of a cycle working with ideal gas are represented in Figure 1.1. The figure shows the cycle in P-h diagram and in T-s diagram. Turbine outlet and compressor outlet temperatures are the same. The isothermal lines are vertical in the P-h diagram because the enthalpy only depends on the temperature, and not on the pressure.

The net specific work, $w$, in an ideal cycle is:

$$
w=\int_{P_{L}}^{P_{H}}\left(v_{t}-v_{c}\right) d P
$$

where subscript $t$ stands for turbine, $c$ for compressor, $H$ for high and $L$ for low. 


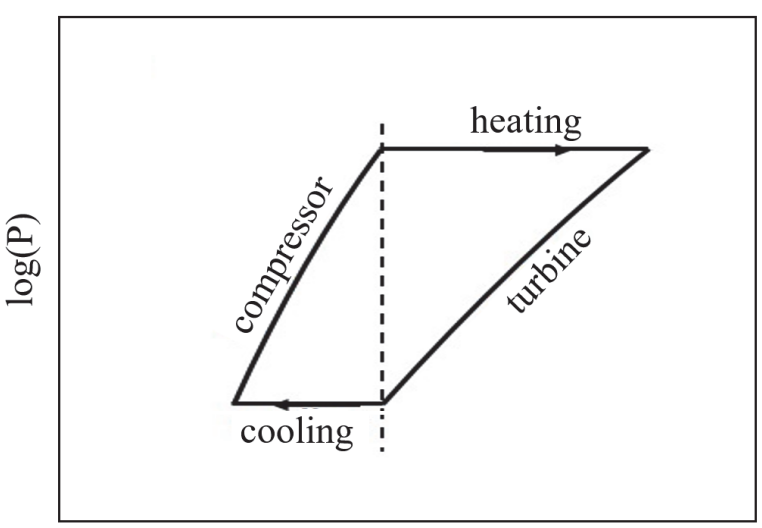

$\mathrm{h}$

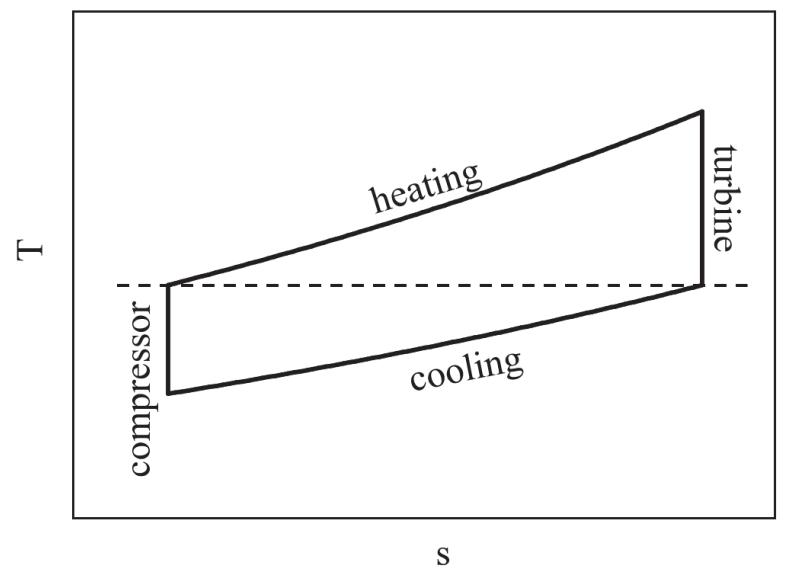

S

Figure 1.1. Ideal closed cycle in diagrams P-h (left) and T-s (tight). Temperatures are equal at compressor outlet and turbine outlet

A way to increase the net specific work of a cycle is to separate as much as possible the compression and expansion isentropic lines in order to increase the difference between the specific volumes $v_{t}$ and $v_{c}$. The essential element in this process is the regenerative intermediate heat exchanger to recirculate the heat excess from the low-pressure side to the high-pressure side. Figure 1.2 shows a scheme of closed regenerative cycle in P-h diagram, valid for both Rankine and Brayton cycles. The fluid in the Brayton cycle would be gas during all the phases, and the fluid would experience phase change during the cooling and/or heating processes in the Rankine cycle. In the latter case, the compression falls under the liquid phase, and the name for the component in charge of this compression is pump. Nonetheless, this report will use the word "compressor" to refer to both compressor and pump with the purpose of generalizing the cycle analysis.

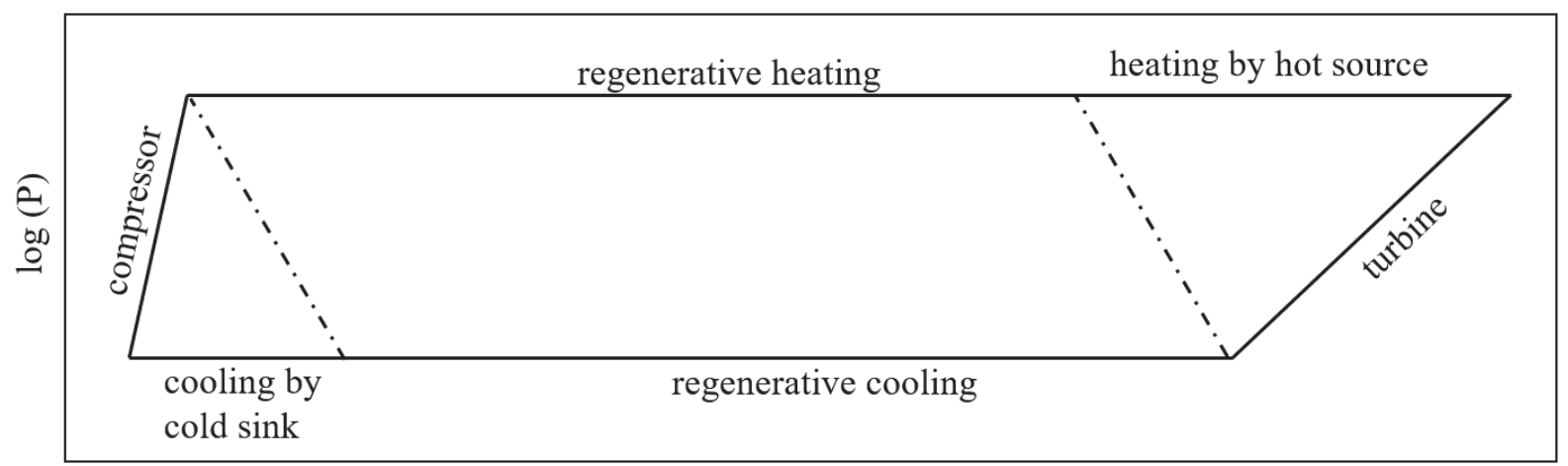

$\mathrm{h}$

Figure 1.2. Regenerative closed cycle in P-h diagram

There are three types of lines in the thermodynamic diagram that dominate the cycle performance: isobars, isentropic lines and isothermal lines. It is necessary to make some 
comments about these lines to understand the cycle performance. Figure 1.3 will help to understand these comments.
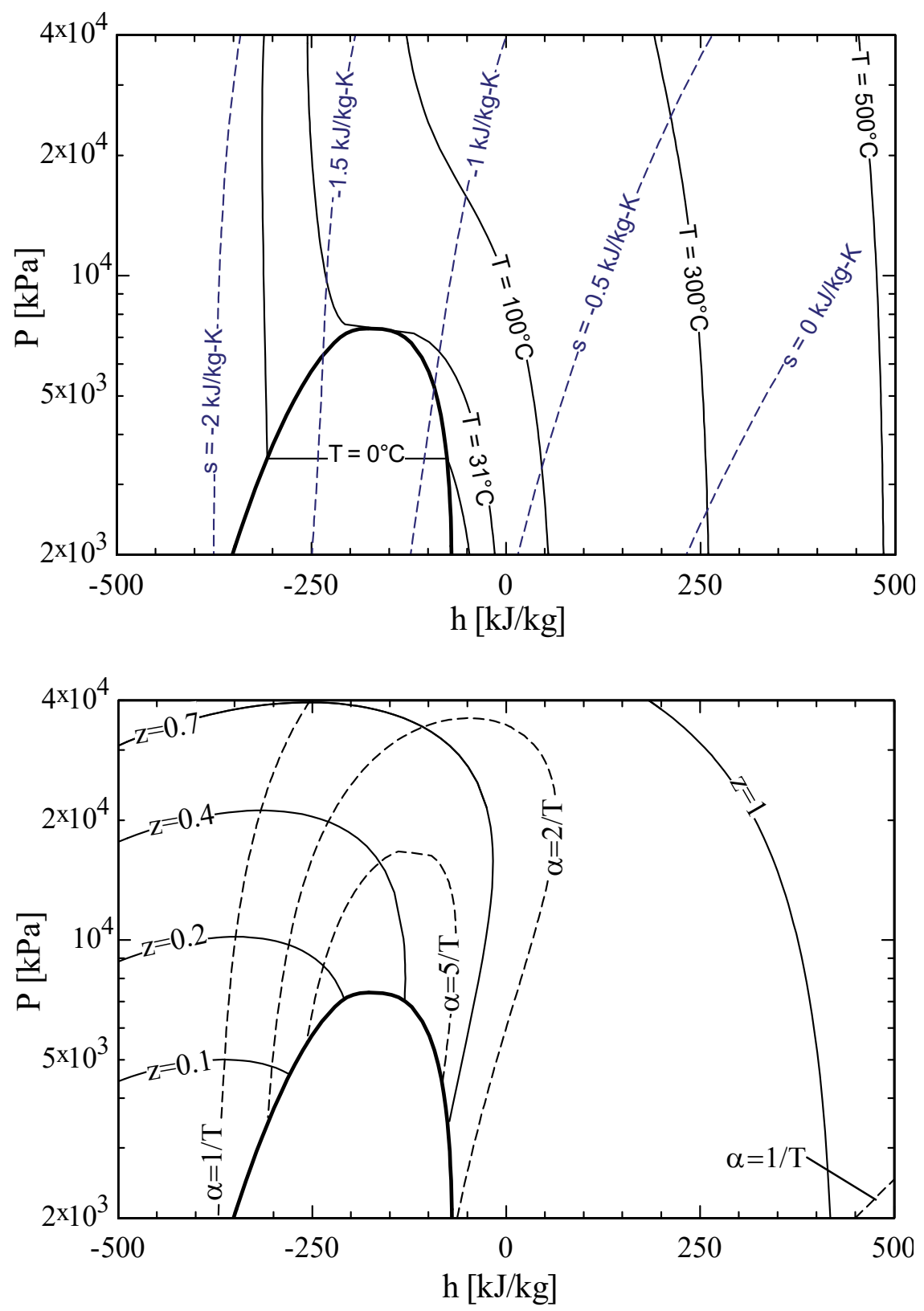

Figure 1.3. P-h diagrams of $\mathrm{CO}_{2}$ with isothermal and isentropic lines (top), and isolines of compressibility factor, $z$, and isobaric expansion coefficient, $\alpha$, in terms of $1 / T$ (bottom)

The enthalpy variation in an isobar is expressed in Equation 1.3, where $c_{P}$ is the specific heat at constant pressure.

$$
d h=c_{p} d T
$$

The isobaric specific heat $c_{p}$ presents very large variations in the thermodynamic region close to the critical point, and it becomes infinity in the critical point (as a memory effect from the 
two-phase domain from liquid to vapor, where latent heat could be understood as a domain with an infinite value of isobaric specific heat). The specific heat variations above the critical pressure (maximum pressure of the biphasic bell in Figure 1.3) involve changes in the isothermal lines slopes of the cold and hot sides of the regenerator. This means that the regenerative heat transfer will not be a uniform process in this region.

The enthalpy variation along an isentropic line is expressed in Equation 1.4.

$$
d h=v d P
$$

In an isentropic line, temperatures in the low- and the high-pressure side are related through the pressure ratio and an isentropic exponent strongly dependent on the isentropic line. For ideal gases, which approximates turbine conditions, this exponent is the adiabatic coefficient $\gamma$, defined as the ratio $c_{p} / c_{v}$ (with $c_{p}-c_{v}=R$ ). The relation between the temperatures of the low- and the high-pressure side, $T_{L P}$ and $T_{H P}$, respectively, can be expressed as:

$$
T_{H P}=T_{L P} r^{(\gamma-1) / \gamma}
$$

where $r$ is the pressure ratio.

The enthalpy variation along an isothermal line is

$$
d h=v_{T}(1-\alpha T) d P
$$

where $v_{T}$ means specific volume at constant temperature and $\alpha$ the dilatation coefficient at constant pressure:

$$
\alpha=\frac{1}{v}\left(\frac{\partial v}{\partial T}\right)_{P}
$$

Isothermal lines do not participate in the global definition of the cycle. However, they convey strict limitations inside the cycle. Isothermal lines mark the limits of the regeneration phase, which will affect the cycle performance. The value of $d h$ is zero for ideal gases because $\alpha=1 / T$. In this case, the lines limiting the regenerative phase in Figure 1.2 would be vertical. However, in the domain near the critical point, the term $\alpha T$ is greater than one. Therefore, the isothermal lines in the P-h diagram are deflected with negative slopes. And at very high pressures, above the Joule-Thompson inversion, the slopes of the isothermal lines are positive.

Once the enthalpy variations are defined along the cycle, the first step is to analyse how to maximize the cycle net specific work. Isentropic lines of compression and expansion are 
located at different regions of the thermodynamic diagram, where the evolution of the volume as a function of the pressure for constant entropy is not the same. This means that the enthalpy variation in compression and expansion, defined in Equation 1.4, will also be different.

At high temperatures far away from the critical point, the fluid behaves like an ideal gas. The ideality of a fluid may be defined by the compressibility factor $z$ :

$$
z=\frac{P v}{R T}
$$

The compressibility factor is mainly used to correlate equations of state, such as the virial equation. However, it is more than a parameter to express a correlation. It is a ratio between internal mechanical energy and thermal internal energy.

Figure 1.4 shows the compressibility factor, $z$, for different pressures and temperatures as a function of density (properly speaking, 100/v) for $\mathrm{CO}_{2}$. According to the definition of $z$ as the ratio of internal mechanical energy and thermal internal energy, the optimum placement for turbine will be located in the left-hand side, upper corner, while the compressor region will lay in the right-hand side, lower bands. The reason is the natural trend of gas molecules to expand, while a compressor does the opposite.

The optimum placement for the compressor in the diagram of

Figure 1.4 is at temperatures below the critical temperature, $T_{c r}$, and pressures below the critical pressure, $P_{c r}$. Compressing a liquid requires smaller powers than compressing an ideal gas. This is the reason of the high efficiency in steam Rankine cycles despite the relatively low turbine inlet temperatures (in comparison to gas turbines). On the contrary, gas turbines utilize air, which behaves as an ideal gas, and large amounts of work are consumed during the compression process. The result is that the thermal efficiency of gas turbines is not significantly higher than the efficiency of steam Rankine cycles despite the much higher turbine inlet temperature (Ahn et al., 2015).

The optimum cycle conditions would be a combination of steam Rankine and gas turbines, with expansion at high temperatures in the ideal gas region and compression in the region of low compressibility factors. The lowest compressibility factors are found in the liquid region. However, some fluids able to achieve high temperatures for the expansion cannot be cooled down under the required temperature to change phase, but can get close to the critical 
temperature, where the values of the compressibility factor are still low, and therefore the compression work will also be highly reduced.

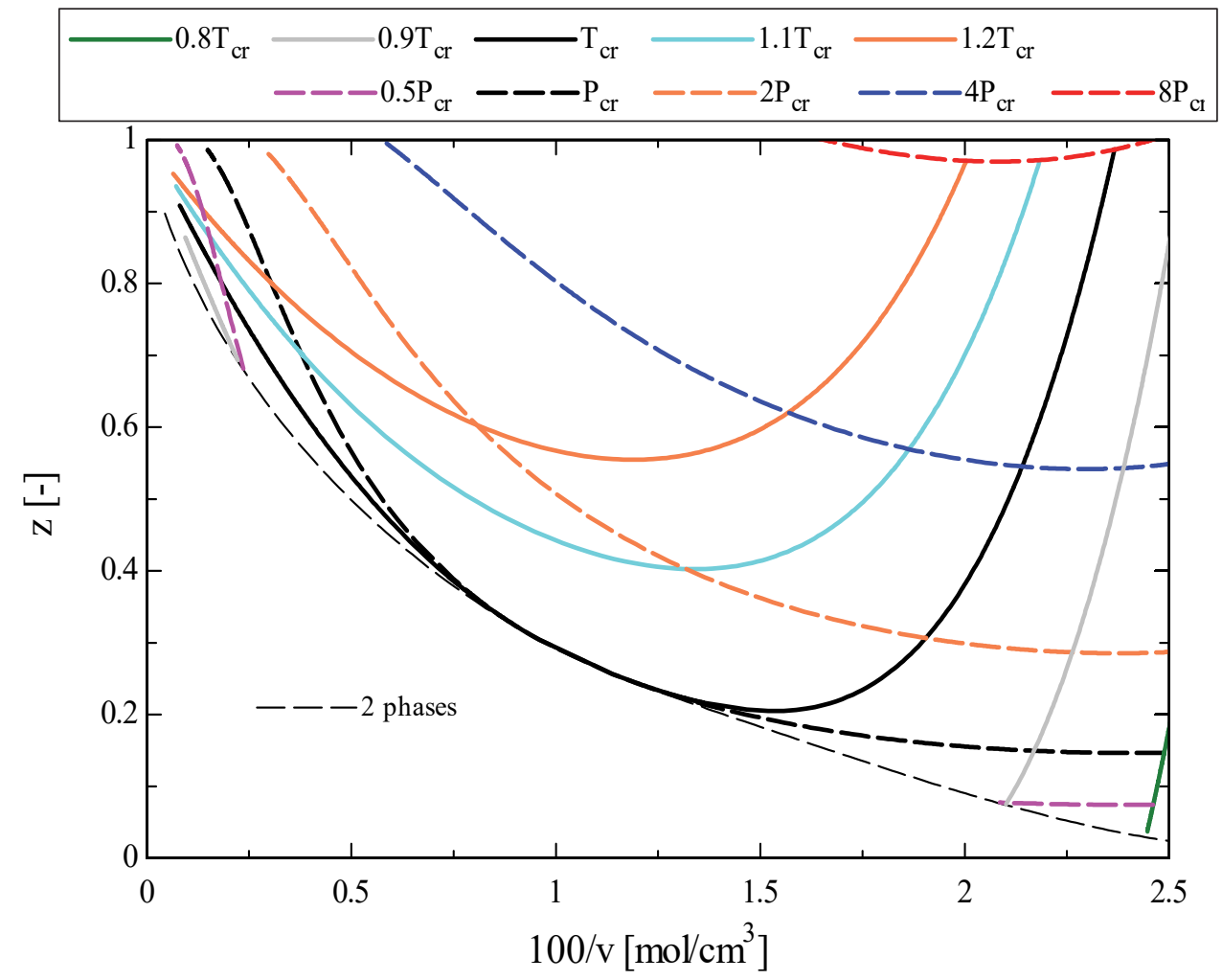

Figure 1.4. Compressibility factor, $z$, of $\mathrm{CO}_{2}$ versus density, $100 / v$, for different values of pressure and temperature (expressed as a function of the critical pressure, $P_{c r}$, and critical temperature, $T_{c r}$, respectively).

Fluids with the compressor working close to the critical point have shown to be very promising in the future of high-efficiency power cycles (Crespi et al., 2017). This region is characterized by large variations of thermophysical properties. Small changes in temperature and/or pressure around the critical point may involve big changes in other thermophysical properties. Therefore, this region must be well characterized to analyse cycles working on it. Due to the high importance of this region, and the continuous reference to it in this document, this region will be called pericritical region, where "peri" stands for "around" in Latin.

\subsubsection{The pericritical region}

Fluids working near the critical point have been present in the nature in aqueous solutions for billions of years, and it was in the late 1800s when scientists started to use this natural process (Pioro and Zvorykin, 2016). Since then several applications have used fluids near the critical point, especially during the last decades, period in which the number of experimental projects 
and publications have grown exponentially. The use of fluids near the critical point does not only consider power cycles, but also many other applications such as advanced materials processing or petroleum extraction (Cabeza et al., 2017).

Despite the wide use of fluids near the critical point, there are still several unsolved topics. One of the main topics is the definition of supercritical fluid. The lack of physical understanding involves that there are different suggestions for the definition of supercritical fluid. The different definitions can be easily understood with the help of Figure 1.5. This figure divides the pressure-temperature (P-T) diagram into four quadrants I - IV, with the critical point common to all of them, defined by the critical pressure $P_{c r}$ and the critical temperature $T_{c r}$. Bellan (2006) considers that supercritical fluids are those in which phase equilibrium is not possible, i.e., fluids in the quadrants II, III, and IV. For Tucker (1999) any state above the critical temperature is supercritical, i.e. quadrants II and III. Pioro and Zvorykin (2016) suggest that supercritical fluids are only in quadrant III and compressed fluids in quadrant IV; nonetheless, Pioro and Zvorykin usually employ the term supercritical fluid to refer to both quadrants. Other authors suggest other definitions (Candel et al., 2006; Younglove, 1982). This document will call supercritical fluid to the fluids working above the critical pressure.

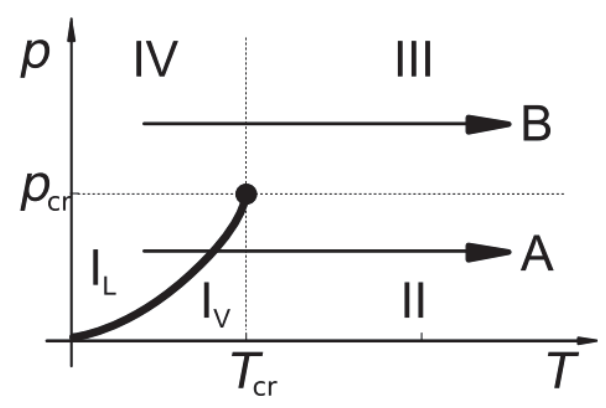

Figure 1.5. Pressure-temperature (P-T) diagram divided into four quadrants with the critical point as common point (Banuti et al., 2017)

Figure 1.5 presents a classical P-T diagram divided into four quadrants and the coexistence line separating liquid and vapor states ( $\mathrm{L}_{\mathrm{L}}$ and IV, respectively). Recently, an extension to the coexistence line called Widom line is also commonly represented in the phase diagram (Sciortino et al., 1997). Figure 1.6 shows the P-T diagram including the Widom line. This line divides liquid-like (LL) and gas-like (GL) supercritical states and exhibits some properties of a phase transition (Banuti et al., 2017). This means that quadrant III can be divided into states (or states-like), despite being at pressures above the critical pressure. A fluid going through the process $\mathrm{A}$ in Figure 1.6 will experience phase change, but with the definition of the 
Widom line, a fluid going through the process B will also transition from a liquid-like to gaslike phase, although in a more continuous manner.

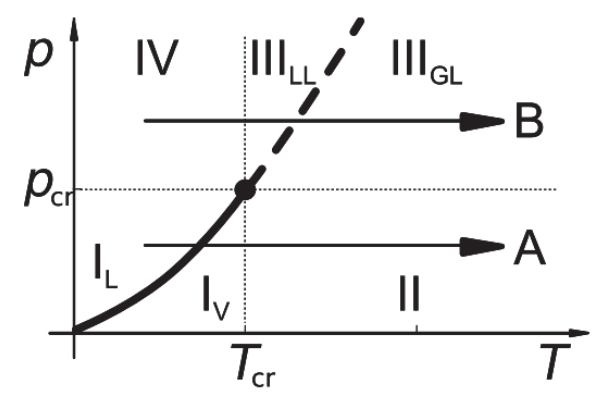

Figure 1.6. Pressure-temperature (P-T) diagram divided into four quadrants with the critical point as common point and the Widom line (dashed), dividing the supercritical quadrant into a liquid-like (subscript LL) and a gas-like (subscript GL) region (Banuti et al., 2017)

The thermo-physical properties undergo sharp variations when crossing the Widom line. In fact, the Widom line is often approximated as the locus of maximum thermodynamic variables such as the isobaric specific heat capacity, $c_{p}$ (Banuti, Raju and Ihme, 2018). The Widom line is also called pseudocritical line when it is composed by pseudo-critical points, where the definition of pseudo-critical point is the point at a pressure above the critical pressure and at a temperature greater than the critical temperature corresponding to the maximum value of specific heat at this particular pressure (Pioro and Zvorykin, 2016).

The sharp variation of thermo-physical properties is characteristic of fluids crossing the Widom line, but also of fluids carrying out a phase change. Figure 1.7 shows the density and the isobaric specific heat capacity of $\mathrm{CO}_{2}$ at subcritical, critical and supercritical pressure as a function of temperature. The main difference between the subcritical and the supercritical values is that the former experiences more abrupt variations, but both get to similar values to the left and right extremes of the figure. The density profiles show that the attained densities at low temperatures converge towards high density values, characteristic of the liquid state, and the differences between the pressures are small. For high temperatures, the densities converge towards low density values approaching their ideal gas values.

The abrupt change of thermo-physical properties in heated supercritical fluids in the vicinity of the pseudo-critical point results in a specific behavior of flow and heat transfer. This behavior is very similar to the boiling occurring at subcritical pressures (Pioro, 2016). When a supercritical fluid with a bulk temperature below the pseudocritical temperature is heated, some layers near the heating surface may reach temperatures above the pseudocritical 
temperature. The fluid near the heating surface will have gas-like (low density) characteristics and the rest of the fluid, liquid-like (high density). Therefore, the gas-like fluid will move from the heating surface in a form of variable density (bubble) volumes. In the same way as in boiling at subcritical pressures, the heat flux will determine if the heat transfer is improved such as in nucleate boiling or deteriorated such as in film boiling (Jackson, 1997).
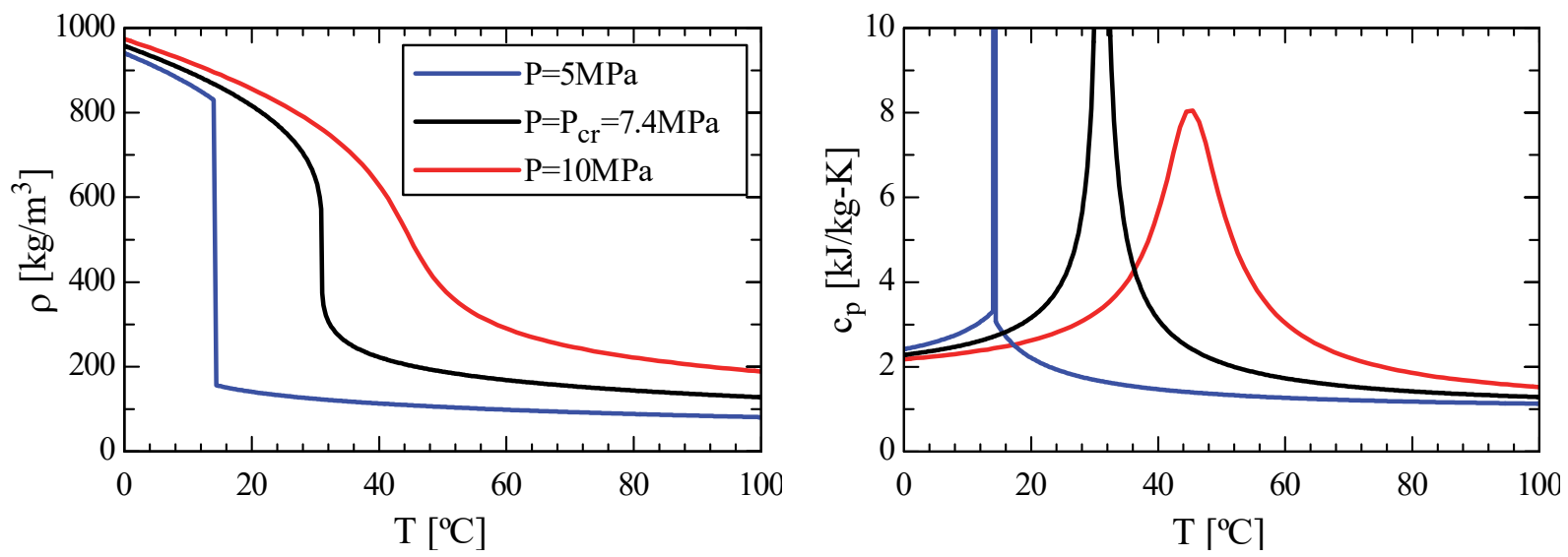

Figure 1.7. Density, $\rho$, and isobaric specific heat capacity, $c_{p}$, of $\mathrm{CO}_{2}$ at subcritical, critical and supercritical pressures as a function of temperature, $T$

In summary, the space of subcritical and supercritical states is divided into distinct liquid and gaseous parts, and the behavior of these liquid and gaseous parts is similar regardless of the pressure. The closer the pressures of the supercritical fluid and the subcritical fluid, the more similar the behavior will be.

\subsubsection{Pericritical power cycles}

The performance of supercritical fluids in power cycles have been proved as an alternative solution for high-performance power generation (Dostal, 2004). The cycles using supercritical fluids are commonly divided into two groups: transcritical and supercritical cycles. These two configurations have many possible layouts that have been proposed during the last decades to increase the cycle efficiency.

The supercritical cycle was proposed by Feher (1968). Feher defined the supercritical cycle as a cycle that operates entirely above the critical pressure of its working fluid and with the compressor close to the critical point. The idea of this cycle was to avoid most of the problems of Rankine and standard Brayton cycles, but retain their advantages. The power required by the compressor in supercritical cycles is relatively small in comparison to 
standard Brayton cycles, but the they conserve its simplicity and compactness (especially using $\mathrm{CO}_{2}$ as working fluid) (Dostal, 2004).

Feher also considered the possibility of pseudo-supercritical cycles, which by then had already been employed in steam power plants. The pseudo-supercritical cycle is a mixture between standard Rankine and Brayton cycles, with the fluid working partially below the critical pressure and partially above it. The working fluid in liquid state is compressed to a pressure above the critical pressure, heated without phase change due to the supercritical state features, expanded in gas state and condensed back to the initial state. Most of these cycles include heat regeneration between the high- and the low-pressure sides. Angelino (1968) studied these cycles with different configurations, finding that they could exhibit better efficiency than reheat steam cycles. These cycles are usually referred to as transcritical cycles.

Some authors compare the differences between transcritical and supercritical cycles (Angelino, 1969; Kim, Kim and Favrat, 2012), but most of researchers study them separately (Crespi et al., 2017; Ma, Liu and Tian, 2013; Sarkar, 2015). The difference between these two cycles is clearly appreciated when the compressor in transcritical cycles works well below the critical pressure and well above it in supercritical cycles. However, when the compressor works in the region close to the critical point, it is more difficult to distinguish if the fluid behaves as a liquid or as a gas and, therefore, the differences between transcritical and supercritical cycles are hardly appreciated.

Despite the definitions made for transcritical and supercritical cycles, in the field of power generation most authors refer to supercritical cycles to those working above the critical temperature with the high-side pressure above the critical pressure but without any restriction for the low-side pressure (Dostal, 2004; Dyreby, 2014). Dostal (2004) proposed this definition with the purpose of avoiding phase change. However, considering the existence of the Widom line, this condition would only be limiting the range of temperatures to consider in the analysis, because in any way, temperatures above but close to the critical line can also experience a behavior similar to phase change.

There are several candidate fluids to work in transcritical and supercritical cycles. The objective is to obtain a fluid that works under ideal gas conditions in the expansion and close to liquid conditions in the compression. This can be achieved with fluids with the critical temperature close to the ambient temperature. Feher (1968) proposed several fluids and selected $\mathrm{CO}_{2}$ due to the moderate value of its critical pressure, its stability and relative inertness (for the temperature range of interest), non-toxicity, abundance and low cost. Since 
then, many researchers have focused on the analysis of supercritical cycles with $\mathrm{CO}_{2}$ (Dostal, 2004; Dyreby, 2014; Sarkar, 2009).

\subsubsection{Supercritical $\mathrm{CO}_{2}$ cycles}

Supercritical $\mathrm{CO}_{2}\left(\mathrm{~s}-\mathrm{CO}_{2}\right)$ cycles have been deeply analysed during the last decades. The $\mathrm{s}-\mathrm{CO}_{2}$ power cycle is considered a promising alternatives to provide high efficiency nuclear (Syblik et al., 2019), solar (Teng and Xuan, 2019) and fossil power plants (Sánchez Villafana and Vargas Machuca Bueno, 2019). Figure 1.8 shows the thermal efficiencies of several power cycles and its possible heat sources as a function of turbine inlet temperature (Ahn et al., 2015). Rankine cycles achieve high efficiency at low turbine inlet temperatures mainly due to the low compression work requirement of water. On the contrary, the gas turbine needs large powers to compress the air and, therefore, despite the turbine inlet temperature is much higher than in the Rankine cycles, thermal efficiency of gas turbines is not significantly higher.

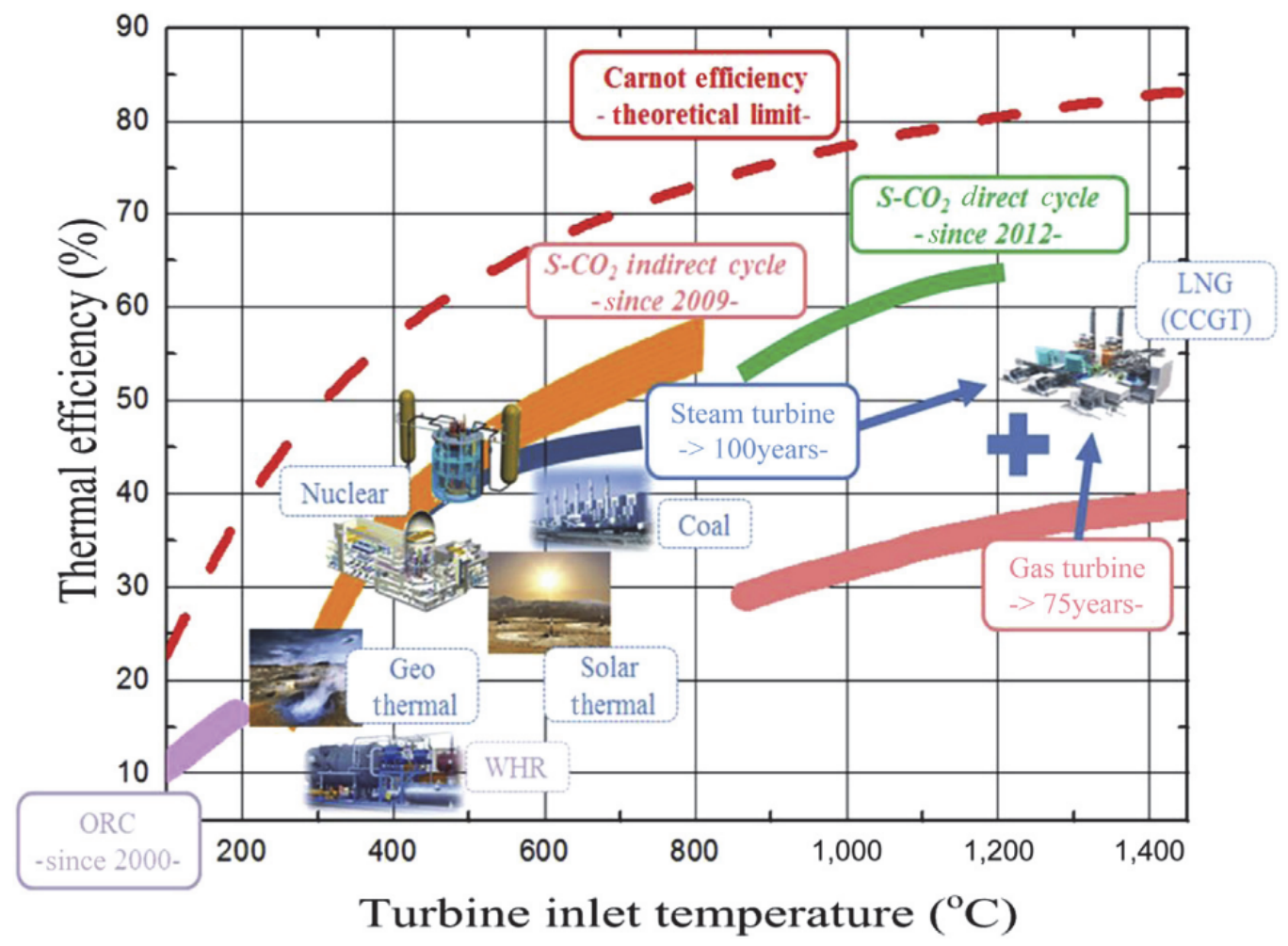

Figure 1.8. Thermal efficiencies of power conversion systems and applications. (Ahn et al., 2015)

$\mathrm{S}-\mathrm{CO}_{2}$ Brayton cycles combine the advantages of both steam Rankine cycle and gas turbine system: the reduced compression work in the liquid-like region (or close to it) and the high turbine inlet temperature that can be utilized with softer material requirements than in steam 
Rankine cycles. The result is that these cycles can achieve higher thermal efficiencies than steam Rankine and gas turbine systems.

The critical pressure of $\mathrm{CO}_{2}$ is $P_{c r}=7.4 \mathrm{MPa}$ and the critical temperature $T_{c r}=31^{\circ} \mathrm{C}$. One of the main advantages of s- $\mathrm{CO}_{2}$ cycles is its reduced compression work in comparison to the work required to compress an ideal gas. This is due the proximity of the compressor inlet temperature to the critical temperature.

The low compression ratio needed by s- $\mathrm{CO}_{2}$ cycles involve that the high-side pressure is commonly set to 20-30 MPa. This means that the fractional pressure drops are low. Moreover, the fluid remains dense throughout the entire power system, which allows more compact heat exchangers and turbines than other cycles. Figure 1.9 shows the difference between the turbine sizes of steam, helium and $\mathrm{CO}_{2}$ cycles. The turbine for the case of s- $\mathrm{CO}_{2}$ involves a great size reduction. In addition to this, $\mathrm{CO}_{2}$ turbines can be designed as a single body, while both steam and helium turbines usually employ more turbine bodies, which increases even more the difference in size.

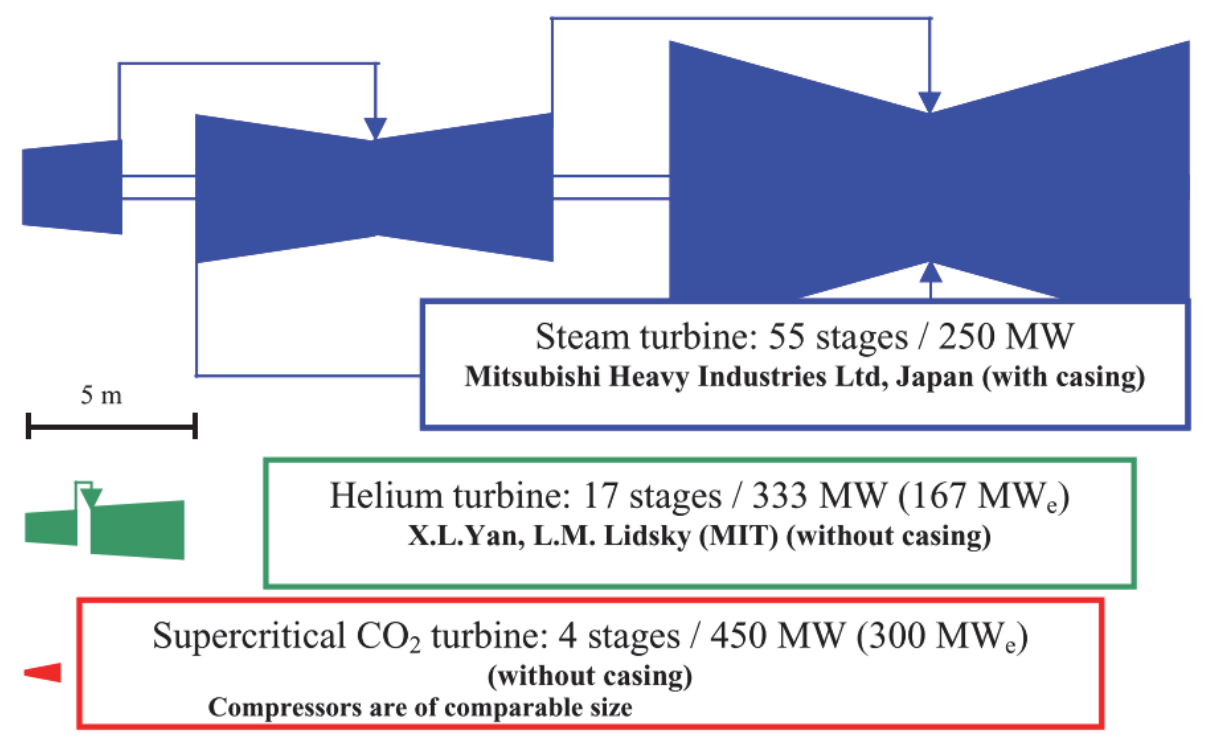

Figure 1.9. Comparison of turbine sizes for steam, helium and $\mathrm{CO}_{2}$ (Dostal, 2004)

One of the main important parts of s- $\mathrm{CO}_{2}$ cycles is the heat regeneration. The big differences between turbine and compressor temperatures and the small pressure ratios involve that large amounts of heat must be recuperated to increase the thermal efficiency. The required volume of conventional shell and tube heat exchangers would be huge and too expensive (Dostal, 2004). Therefore, s- $\mathrm{CO}_{2}$ cycles need to use compact heat exchangers. Printed Circuit Heat Exchanger (PCHE) are suggested as the most suitable alternative due to its capacity to withstand high pressures and high temperatures (Dostal, 2004). 
The regeneration phase of $\mathrm{s}-\mathrm{CO}_{2}$ cycles exhibits large internal irreversibilities due to a mismatch between the specific heats of the low- and high-pressure sides (Pérez-Pichel et al., 2012), which has important drawbacks in the thermal efficiency. Adding a second compressor in parallel to the main one is one of the most common solutions for this problem. Despite the extra compression work, these cycles achieve higher efficiencies than simple regenerative s- $\mathrm{CO}_{2}$ cycles (Sarkar, 2009). This layout is called recompression cycle, and it has been highlighted by many researchers due to its high efficiency (Dunham and Iverson, 2014).

In addition to recompression cycles, there are many other alternative layouts with high potential to increase the cycle performance (Ahn et al., 2015). However, the more sophisticated the configuration, the more expensive will be. Therefore, despite there are layouts with higher efficiency than simple regenerative cycles, the latter seem to be an attractive option due to its simplicity.

The optimum cycle configuration will depend on the specific characteristics of each application. For example, in CSP plants, where there is lack of water, the s- $\mathrm{CO}_{2}$ cycle must be cooled with air, which on the one hand increases the size of the heat exchangers, and on the other increases the compressor inlet temperature. In this case, one of the few estimations of cost in $\mathrm{s}-\mathrm{CO}_{2}$ cycles carried out by Ho et al. (2015) obtains that the simple regenerative cycle is the cheapest option.

\subsubsection{Concentrating Solar Power}

A CSP plant is a thermal power plant divided into three main subsystems: power block, solar field and thermal energy storage. The solar field is composed of a concentrator in charge of reflecting the solar radiation and a receiver in charge of absorbing it. The power block converts the collected thermal energy into electricity. And any excess of thermal energy can be stored in the thermal energy storage and used afterwards to produce electricity when necessary. Figure 1.10 shows a scheme of a CSP plant with the mentioned elements.

The power block in current CSP plants is a steam Rankine cycle, and the thermal storage (if there is) is a two-tank storage with molten salts inside. However, the solar field and the heat transfer fluid (HTF) vary from one plant to another (IRENA, 2012). The roadmap for the future of CSP plants marked by the National Renewable Energy Laboratory (NREL) suggests the use of dry-cooled (cooled by air) s- $\mathrm{CO}_{2}$ cycles for the power block and a solar tower receiver with an heliostat field for the solar field (Mehos et al., 2017), such as the one shown in Figure 1.10. The s- $\mathrm{CO}_{2}$ cycle requires thermal power at high temperatures that the solar 
tower can achieve with high efficiencies. The type of receiver in the solar tower is still unclear, and so is the type of thermal energy storage, which will depend on the type of receiver.

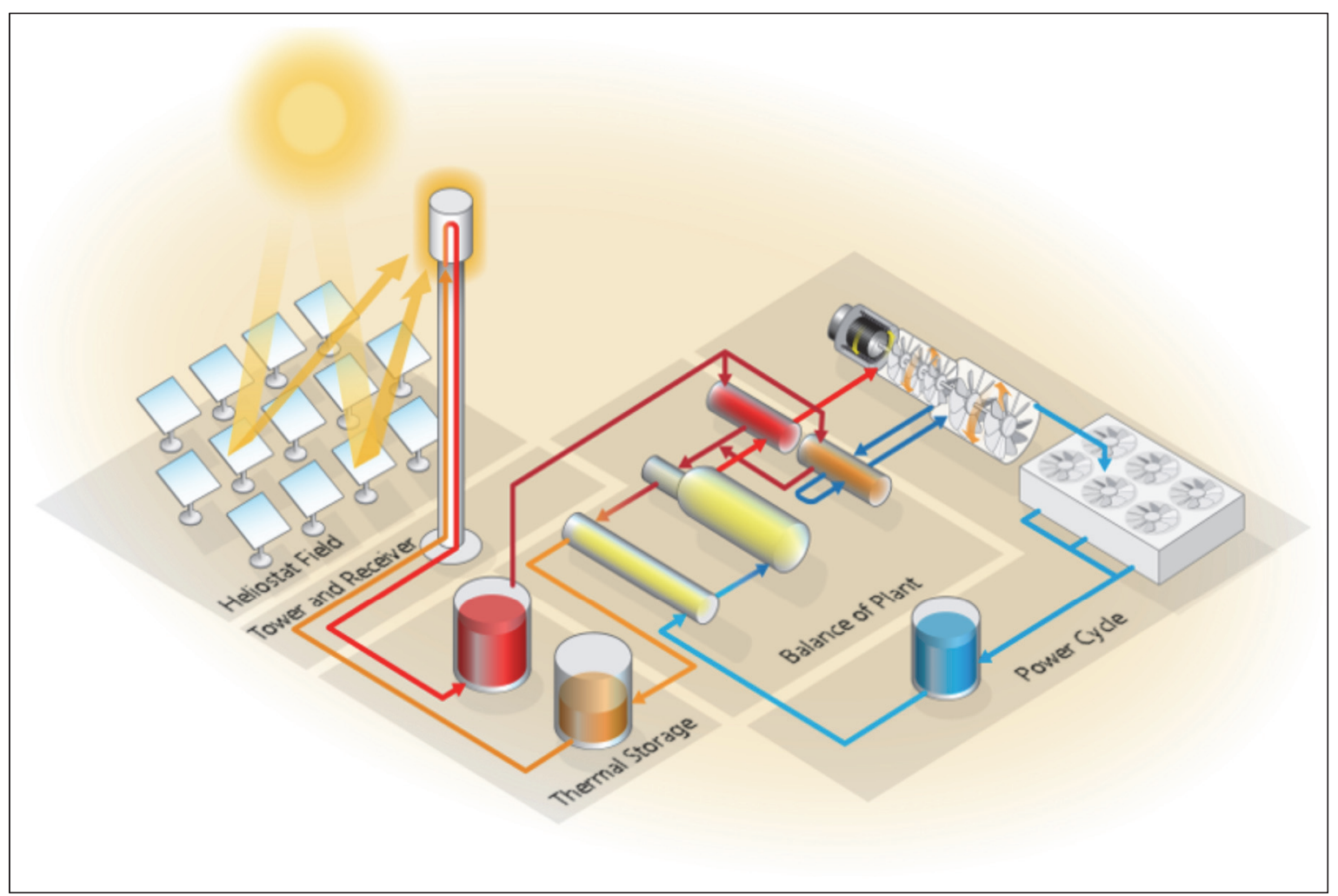

Figure 1.10. Scheme of a CSP plant with solar tower (NREL, 2017).

The main advantage of CSP plants is the low cost of the thermal energy storage, which allows to generate electricity whenever is needed at reasonable prices. This price is still higher than the price of conventional thermal power plants with fossil fuels, but it is lower than its competitors in the field of renewable energies. Figure 1.11 shows the Levelized Cost of Electricity (LCOE) of a CSP plant with thermal energy storage (TES) and a photovoltaics (PV) plant with batteries as a function of the storage size, measured in hours (GonzálezPortillo, Muñoz-Antón and Martínez-Val, 2017a). The hours of storage refer to the time that these plants can operate at nominal power without sun energy. When the plant has no storage, PV is 2.5 cheaper than CSP. However, if the plant needs to produce electricity for more than 3 hours during the night, the option of CSP with TES becomes cheaper than PV with batteries.

In Figure 1.11, the solar field area has been optimized for each storage size in order to achieve the lowest LCOE. The optimum plant configuration is highly influenced by the relation between the costs of TES and solar field (González-Portillo, Muñoz-Antón and Martínez-Val, 2017b). 


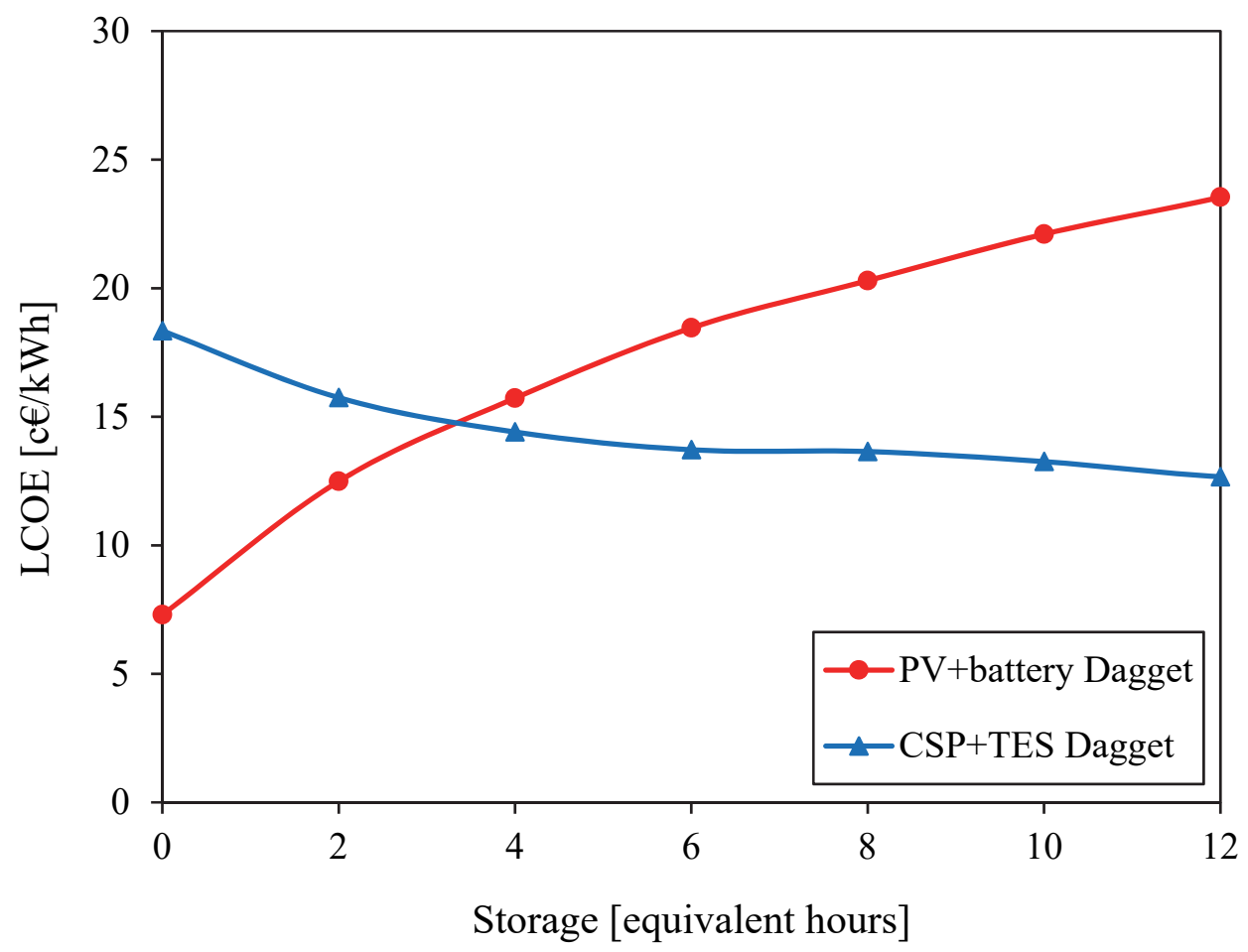

Figure 1.11. LCOE (in $\mathrm{c} € / \mathrm{kwh}$ ) as a function of storage size (in hours) with PV+battery and CSP+TES (González-Portillo, Muñoz-Antón and Martínez-Val, 2017a)

Despite the lower LCOE of CSP plants with big storages, this technology needs to reduce its cost in order to become more competitive against conventional energies, and against possible reductions of battery prices. The main cost of a CSP plant is the solar field. Therefore, it is essential to reduce the cost of this part in order to reduce the LCOE of CSP plants. There are two ways to achieve this objective: reduce the size of the solar field and reduce its cost. Both options require a design that consider the interactions between the different elements of the CSP plant.

In the design of a CSP plant, it is essential the coherent integration of solar field and power block. The purpose of this integration is, on the one hand, to achieve high system efficiencies and, therefore, reduce the size of solar field; and on the other hand, to employ the cheapest type of solar field that can be adapted to the power block requirements.

Consider as an example an ideal system with Carnot efficiency in the cycle and a black body as receiver. The system efficiency is expressed in Equation 1.9, and the Carnot and receiver efficiencies in Equations 1.10 and 1.11, respectively, where $T_{\text {cold }}$ and $T_{\text {hot }}$ are the minimum and the maximum temperatures of the system, $\sigma$ the Stefan-Boltzmann constant $\left(5.67 \times 10^{-8}\right.$ $\left.\mathrm{W} / \mathrm{m}^{2} \mathrm{~K}^{4}\right), G$ the solar heat flux and $C$ the concentration ratio. 


$$
\begin{gathered}
\eta_{\text {syst }}=\eta_{C} \eta_{\text {rec }} \\
\eta_{C}=1-\frac{T_{\text {cold }}}{T_{\text {hot }}} \\
\eta_{\text {rec }}=1-\frac{\sigma T_{\text {hot }}^{4}}{C \cdot G}
\end{gathered}
$$

Figure 1.12 represents these efficiencies as a function of the temperature $T_{h o t}$ for concentration ratios $C=10, C=100$ and $C=1000$. While the power block efficiency increases with the temperature (following Carnot's law), the receiver efficiency decreases. The combination of Carnot and receiver efficiencies involves a maximum in the system efficiency, which depends on the concentration ratio. Concentration ratio, which is imposed by the solar field, and maximum temperature, which is generally imposed by the maximum achievable HTF temperature, are highly related. Systems with high temperature need solar fields with high concentrations. NREL proposes the combination of $\mathrm{s}-\mathrm{CO}_{2}$ cycles with solar towers because the purpose of s- $\mathrm{CO}_{2}$ cycles is to reach high temperatures, and solar towers can supply them efficiently with high concentration ratios.

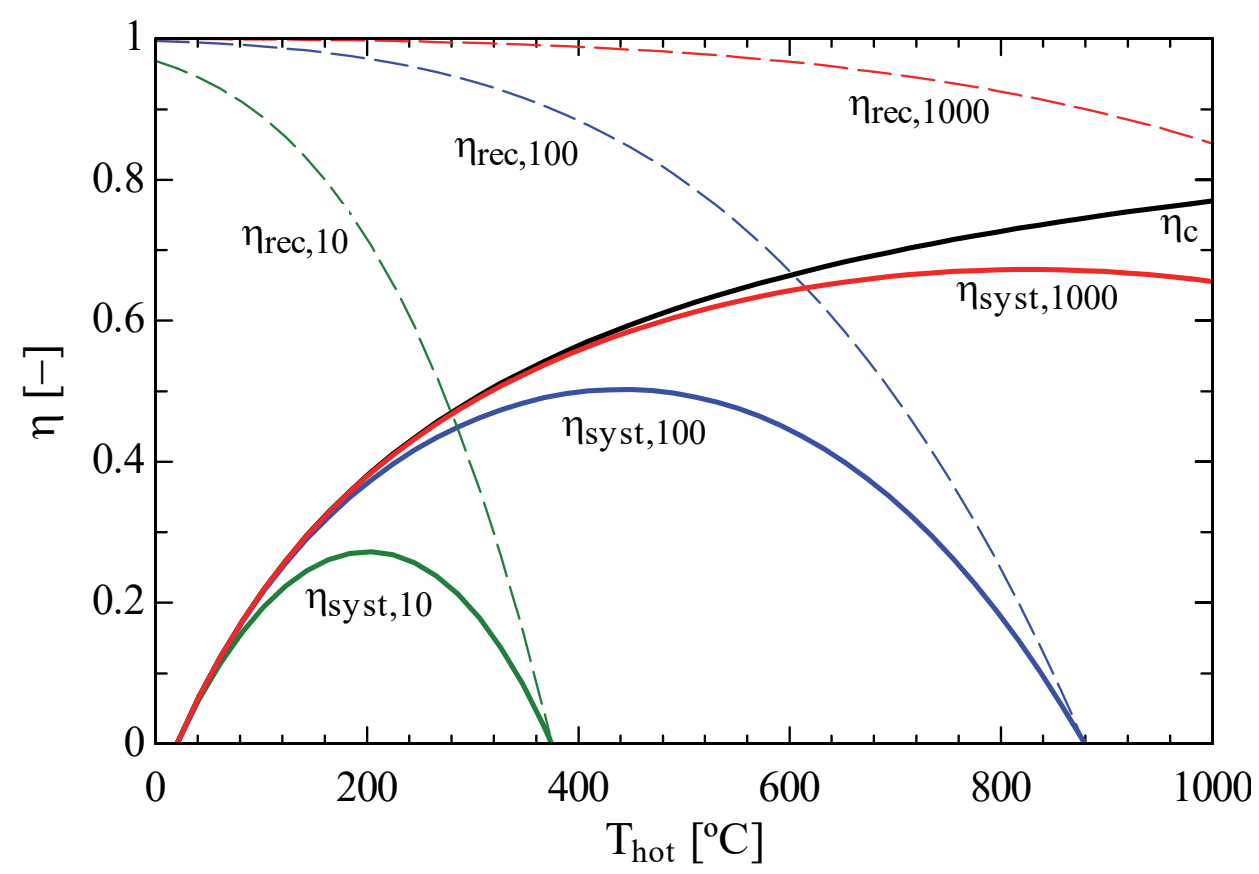

Figure 1.12 . Receiver efficiency, $\eta_{r e c}$, Carnot efficiency, $\eta_{C}$, and system efficiency, $\eta_{s y s t}$, as a function of maximum system temperature, $T$ hot, with the subscript indicating the concentration ratio $C=10, C=100$ and $C=1000$

If the maximum system temperature $T_{h o t}$ is low, the system efficiency with large and small concentration ratios is similar. However, higher concentration ratios generally involve more 
complex and expensive systems. Thus, solar fields with small concentrations will be preferred when the maximum system temperature is low (Muñoz, Martinez-Val and Ramos, 2011).

There are several types of solar field. The concentration ratios that the solar field can achieve will determine the range of fluid temperatures where they will better fit. It has been said that solar towers can achieve large concentration ratios and therefore high efficiencies at high temperatures. On the other hand, other types of solar field with lower concentration ratios, such as parabolic trough or linear Fresnel collectors, adjust better to lower temperatures (Muñoz, Martinez-Val and Ramos, 2011). There is even the idea proposed by Muñoz et al (2009) to reconfigure the concentration ratio along the heating to adapt the solar heat flux to the temperature variation along the receiver. This new concept was called "Solar Boiler" and consisted in an expanded central tower in which the solar heat flux is accommodated along the receiver tubes. Adjusting the concentration levels to the actual needs of the thermal application can be an efficient tool for increasing the overall performance and lower the costs.

\subsection{Objectives}

The main objective of this work is to develop a coherent design between thermodynamics and heat management for CSP plants, with the final purpose of reducing the system cost. The pathway towards this objective will be done in small steps to ensure the full cover of all the relevant aspects. The thermodynamic analysis will be the basis of the whole process, and the integration with the CSP basics will match the specific characteristics of pericritical cycles with the specific characteristics of solar thermal energy.

A systematic and comprehensive analysis of the pericritical region (surroundings of critical point) will be the first step in the proposed pathway. The characterization of this region is essential to properly analyze the cycles working in this region. Reduced properties will guide the thermodynamic analysis of pericritical cycles in order to abstract the results from the fluid. The generic fluid must have a critical temperature close to the ambient temperature (either below or above) in order to be able to place the compressor in the pericritical region, and therefore being a candidate to work in pericritical cycles.

The thermodynamic analysis of pericritical cycles will present the novelty of studying supercritical and transcritical cycles in a single analysis, with the same parameters defining them. This allows to generalize the characterization of all the cycles working in the surroundings of the critical point without the limitation that may involve the study of different 
regions separately. The cycle characterization will follow the idea of the Widow line as a continuation of the phase change. The behavior of the cycles will be better characterized by the locus of the compressor with respect to this line than by the pressure. The Widow line and the phase change will be essential in the identification of the best suited operating conditions for pericritical cycles.

An important limitation to increase the efficiency of pericritical cycles is the large irreversibility along the regeneration phase. The most common layout to reduce this problem is the recompression cycle, which reduces the irreversibilities by means of adding a compressor. The objective of this work is to reduce the problem of irreversibilities by managing the heat during regeneration with heat sources at different temperatures, having in mind the behavior of CSP plants. The result is a promising layout that will be called multiheating cycle. The best suited operating conditions of multi-heating cycles will be analysed, and the results will be compared with its primary competitor: the simple pericritical regenerative cycle.

The comparison between simple and multi-heating cycles will consider different positions for the compressor in the pericritical region by means of reduced temperatures and pressures. This analysis allows to generalize the analysis of these cycles. However, these positions will highly depend on the critical temperature and the critical pressure of the fluid. Therefore, once a generic optimum cycle layout is selected, it is important to refine it and perform a more detailed analysis, which involves the selection of a fluid. $\mathrm{CO}_{2}$ is selected for working in pericritical cycles due to its well-known performance at high temperatures and its affordable pressures.

The management of the heat regeneration phase is the fundamental difference between simple and multi-heating cycles. The complete characterization of this phase requires the analysis of intermediate heat exchangers, in charge of this process. It is necessary to size these heat exchangers in order to develop a preliminary plant footprint. The influence of the size on the cycle performance will be compared under different conditions for simple and multi-heating cycles.

The final step of the pathway to match thermodynamics and heat management is to integrate the multi-heating cycle in an actual CSP plant. While a simple regenerative cycle needs thermal power in a specific range of temperatures, a multi-heating cycle needs it in several ranges of temperature. Each range of temperature will fit better with a different concentration ratio. Therefore, a specific solar field will be proposed ad hoc to match the specific 
characteristics of multi-heating cycles and CSP. The performance of the system with multiheating will be compared with a system with simple cycle and a solar tower to highlight the benefits of multi-heating. Nonetheless, the final purpose of a design following the principles of thermal coherence is to reduce the costs, in this case of the electricity generation. Therefore, the end of this work consists on an economic analysis to quantify the benefits of the proposed multi-heating system with respect to a system with solar tower and pericritical simple regenerative cycle.

\subsection{Methodology}

The computational models used in this work are developed in EES (Engineering Equation Solver) (Klein, 1992). EES is a general equation-solving program that can numerically solve thousands of coupled non-linear algebraic equations, which can be entered in any order. The library of fluid properties is utilized to obtain the thermophysical properties of $\mathrm{CO}_{2}$. Moreover, the optimizer provided by EES is used for some of the calculations. EES is a powerful tool for the simulation of cycles and CSP plants. Proof of it is its wide use in previous investigations of thermodynamic cycles and solar thermal power plants (Akbari and Mahmoudi, 2014; Dunham and Iverson, 2014; Forristall, 2003; Turchi et al., 2012).

The equations used in the computational models are explained during the course of the document to guide the analysis. The explanation of equations along the document allows to structure the report as a pathway that exposes new ideas according to the findings. Most of the equations can be easily implemented in EES and therefore there is no need of paying attention to the code. The implementations that require any additional specification are explained in its respective section.

Figure 1.13 shows the modules of the computational model developed in EES. Pericritical cycle and solar field are developed in different modules. The integration of both will compose the model of the CSP plant. Simple cycles will be coupled to standard solar fields and multiheating cycles to solar fields designed ad hoc to integrate multi-heating. Besides the modules developed to simulate the CSP plant, the module of pericritical cycles includes a part focused on the thermodynamic analysis. This module defines new thermodynamic coefficients to guide the cycle characterization. The explanation of these models together with the corresponding analysis is structured in different chapters. 


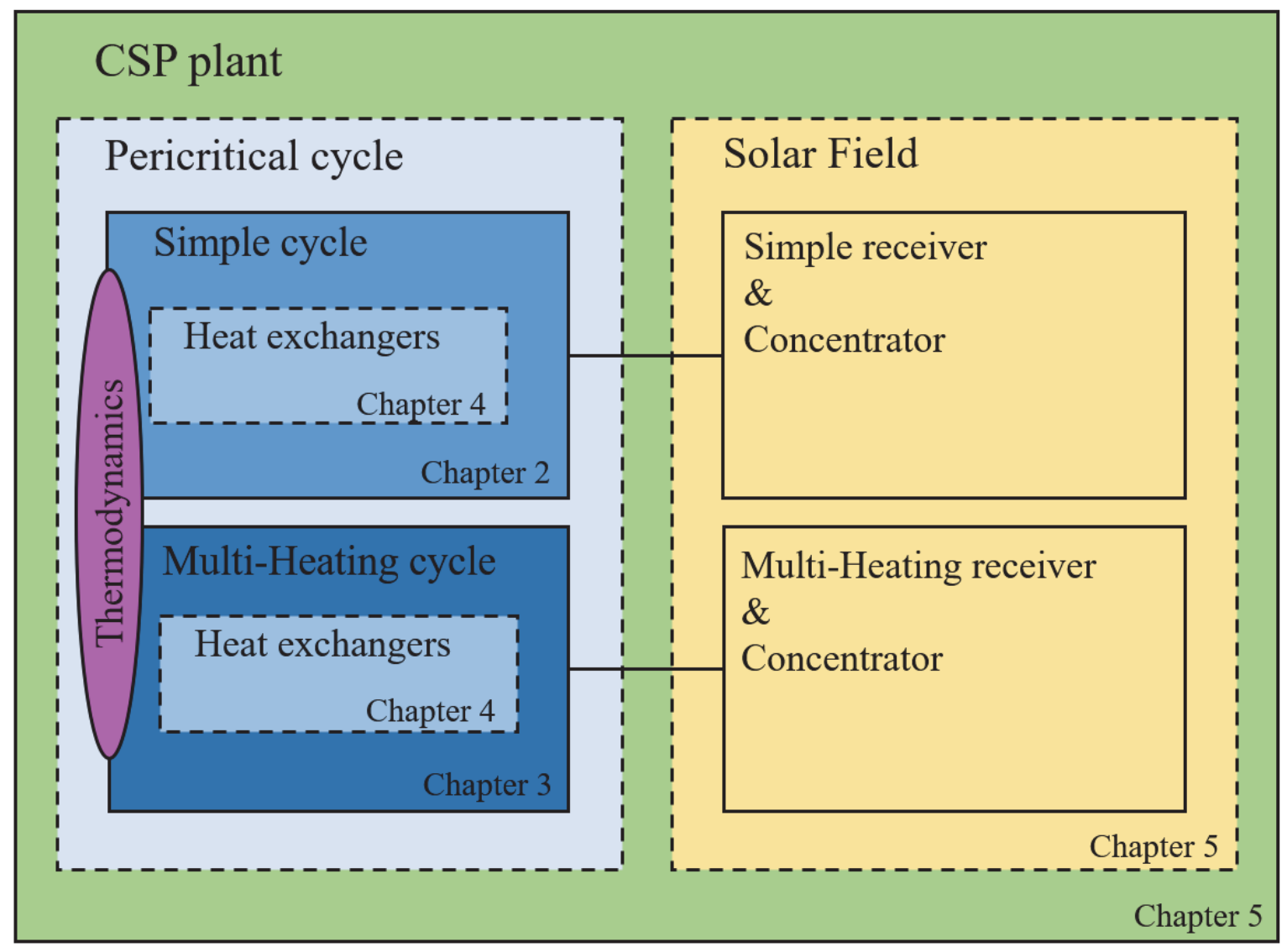

Figure 1.13. Structure of the computational model

\subsection{Structure}

The present document is divided into six chapters including the introduction.

Chapter 2 contains a thermodynamic mapping of pericritical cycles. New thermodynamic coefficients are defined for a better guidance in the cycle characterization. The thermal efficiency of pericritical cycles is analysed for different conditions of pressure and temperature, and for cases with different component inefficiencies. This chapter also describes the general features that a fluid must have to work in pericritical cycles, and some examples are presented.

Chapter 3 presents the multi-heating cycle and investigates its behavior. The irreversibilities found during the regeneration in pericritical cycles are reduced in the multi-heating cycle by adding heats at different temperatures. Two multi-heating layouts are proposed. The benefits of these cycles are analysed by means of energetic and exergetic analysis. These benefits depend on pressure and temperature conditions, and on the inefficiency of components. 
While Chapters 2 and 3 attempts to analyse pericritical cycles for a generic fluid, Chapter 4 focuses on the use of $\mathrm{CO}_{2}$ as working fluid. This chapter analyses the effect of the heat exchanger size on the performance of simple and multi-heating pericritical cycles for different compressor inlet temperatures.

Chapter 5 deals with the integration of multi-heating $\mathrm{CO}_{2}$ cycles in Concentrating Solar Power plants. A suitable solar field is developed specifically for the multi-heating cycle. The influence of temperature and concentration ratio on the receiver efficiency is analysed. The performance of the system with multi-heating is compared to a system with simple cycle and solar tower. A techno-economic analysis investigates the cost of electricity and the critical components of these systems.

Chapter 6 summarizes the most important results, highlights the major conclusions and recommends future work. 


\section{THERMODYNAMIC MAPPING OF PERICRITICAL CYCLES}

In thermodynamics, a critical point is the end point of a phase equilibrium curve. In this work, the term critical point will refer specifically to the liquid-vapor critical point. Fluid properties around the critical point experience strong variations. Thus, two fluids with similar conditions of pressure and temperature close to this point may have different behavior. Figure 2.1 shows the names commonly associated to the different behaviors around the critical point depending on the pressure and temperature (Pioro, Mahdi and Popov, 2017). This chapter analyses the differences and similarities of all these "states" from a thermodynamic point of view. The region around the critical point will be called pericritical region, where "peri" means "around" in Latin.

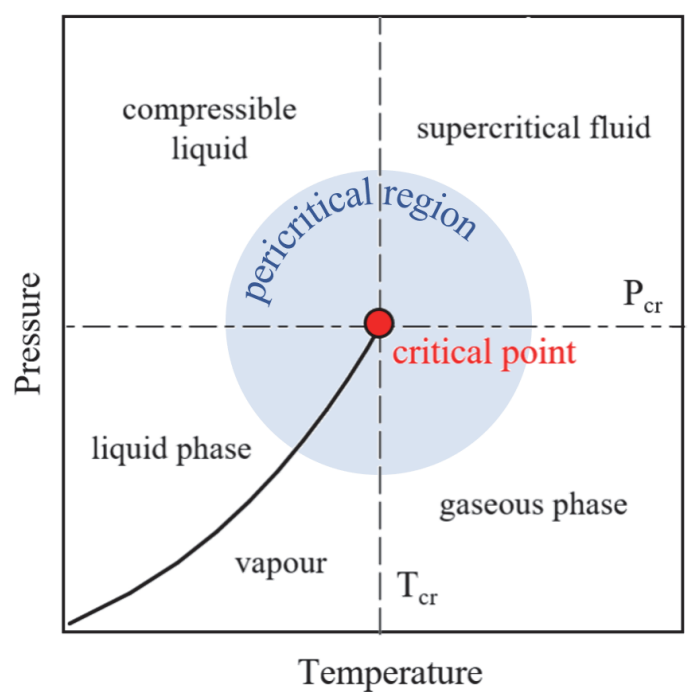

Figure 2.1. Fluid "states" in a pressure-temperature diagram 
The purpose of analysing the pericritical region is to characterize the thermodynamic cycles with the compressor working in this region. These cycles have been generally called supercritical if the whole cycle works at pressures above the critical pressure, and transcritical, if the high-pressure side is above the critical pressure but the low-pressure side is below. The characterization of the pericritical region allows to unify the analysis of supercritical and transcritical cycles, generally studied separately, under a single analysis.

Pericritical cycles have been deeply studied for the last decades (Angelino, 1969; Dostal, 2004; Feher, 1968; Kim, Kim and Favrat, 2012). Besides the classification into supercritical and transcritical, these cycles are also classified into Brayton cycles, when the working fluid is a gas, and Rankine cycles, when there is condensation in the low-pressure side. In transcritical cycles, the cycle is called Rankine if the compressor inlet temperature is below the saturation temperature, and Brayton if it is above. In supercritical cycles, there is no phase change, so they are classified as Brayton. However, the fluid behaves like a liquid when the Brayton cycle works close to the critical point. Previous investigations show, in fact, that there are many similarities between sub- and supercritical states, especially when the fluid works close to the critical point (Banuti et al., 2017). Therefore, this chapter avoids all the mentioned classifications and study all the cycles working around the critical point under the same analysis. All these cycles will be characterized with the same parameters, so they will receive the same name: pericritical cycles.

This chapter is an adaptation from the author's publication Thermodynamic mapping of power cycles working around the critical point (González-Portillo, Muñoz-Antón and Martínez-Val, 2019).

\subsection{The pericritical region}

\subsubsection{Domains of the compressibility factor}

In the energy balance of a cycle, enthalpy variations are the dominant terms. The general form of these variations can be written as:

$$
d h=c_{p} d T-v_{T} f_{p} d P
$$

where the coefficient $f_{P}$ is equal to $\alpha T-1$. This new non-dimensional coefficient will be called logarithmic factor of isobaric expansion. 
The logarithmic factor of isobaric expansion, $f_{P}$, can also be expressed as a function of the compressibility factor, $z$ :

$$
f_{P}=\left(\frac{\partial \operatorname{Ln} Z}{\partial \operatorname{Ln} T}\right)_{P}
$$

Figure 2.2 shows the P-h diagram of $\mathrm{CO}_{2}$ with isolines of $z$ and $f_{P}$. The positive values of $f_{P}$ mean that increasing the temperature will increase the compressibility factor too. In the enthalpy limits on the sides, the value of $f_{P}$ tends to zero. The temperature barely affects the compressibility factor in the liquid region (to the left) and it does not affect at all in the ideal gas region (to the right).

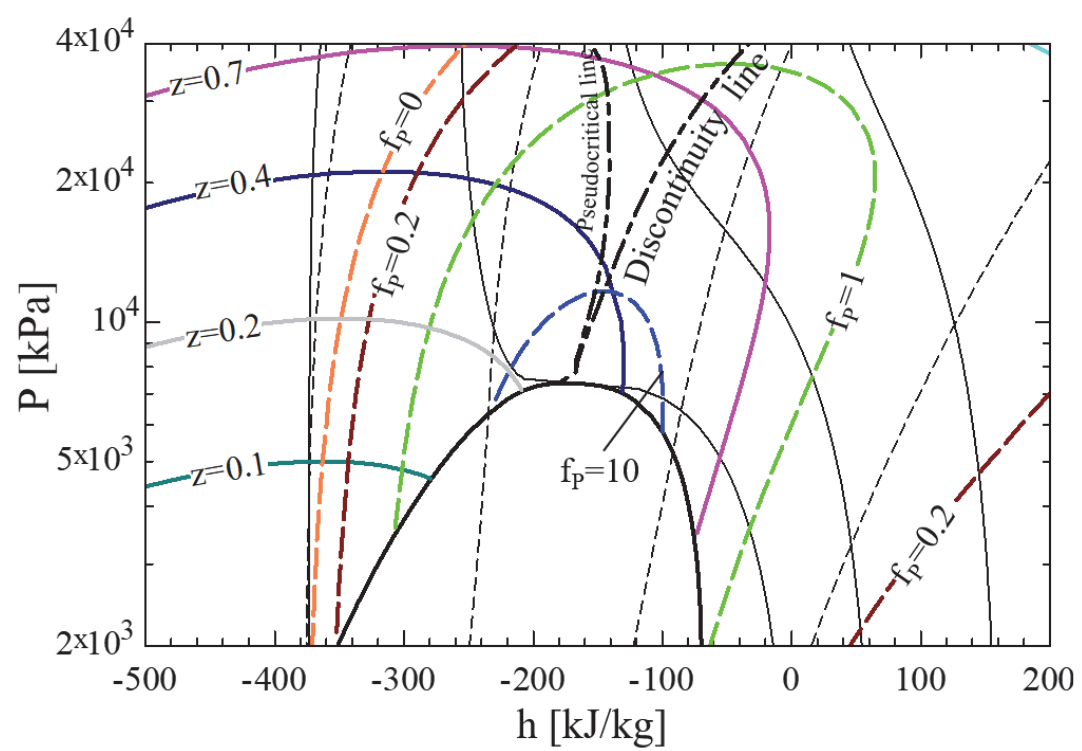

Figure 2.2. P-h diagram of $\mathrm{CO}_{2}$ around the critical point showing isolines of compressibility factor, $z$, and logarithmic factor of isobaric expansion, $f_{P}$.

There is a maximum logarithmic factor of isobaric expansion for each pressure. The line connecting these points of maximum $f_{P}$ will be called "discontinuity line". Note that the part of the discontinuity line above the critical pressure could also be called Widom line (Sciortino et al., 1997). However, the discontinuity line contains also the biphasic bell, where the logarithmic factor of isobaric expansion is infinite due to the abrupt variation of compressibility factor. Thus, the discontinuity line contains values above and below the critical pressure.

The discontinuity line separates two regions with different values of compressibility factor. The variation of the compressibility factor is abrupt below the critical pressure and continuous above it. Although in a continuous manner, the pressures just above the critical pressure experience large compressibility factor variations. This means that, although there is no phase 
change (at least in the traditional way), the discontinuity line separates two regions where the fluid behaves in different ways: liquid-like and gas-like. Figure 2.3 shows a scheme of how the discontinuity line separates these two regions in a pressure-temperature diagram.

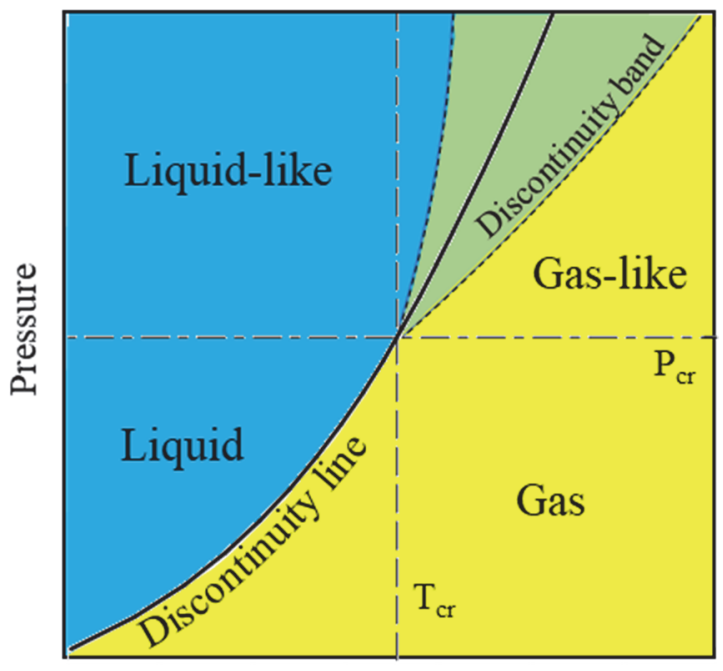

Temperature

Figure 2.3. Fluid states separated by the discontinuity line in a pressure-temperature diagram.

The difference between liquid-like and gas-like regions is less appreciable as the pressure increases. Moreover, when the pressure increases, the variation of the compressibility factor happens along a bigger range of temperatures. This implies that the change from liquid-like and gas-like, or vice versa, instead of being defined by a line, is defined by a band. This band will be called discontinuity band. Figure 2.3 shows the discontinuity band in green.

The logarithmic factor of isobaric expansion is not the only thermodynamic variable suffering strong variations close to the critical point. The specific heat, for example, behaves in a similar way. The points above the critical pressure and at a temperature corresponding to the maximum specific heat at a particular pressure are called pseudocritical points (Pioro and Zvorykin, 2016). These points form the pseudocritical line, which is shown together with the discontinuity line in Figure 2.2.

Pseudocritical and secondary lines represent the highest values of two different thermodynamic properties, $f_{P}$ and $c_{P}$. Therefore, they have a different arrangement in Figure 2.2. This difference is larger at higher pressures where liquid-like and gas-like behaviors present less differences. However, these two lines are very similar close to the critical point, where the values of $f_{P}$ and $c_{P}$ tend to infinity. The relation between these two terms can be found by applying the equality of second derivatives (Bejan, 2006) to Equation 2.1. Equation 2.3 shows this relation. 


$$
\left(\frac{\partial c_{P}}{\partial P}\right)_{T}=-\left(\frac{\partial\left(v_{T} f_{P}\right)}{\partial T}\right)_{P}
$$

Other coefficient that will help to understand pericritical cycles is the logarithmic factor of isothermal expansion, $f_{T}$, defined as:

$$
f_{T}=\left(\frac{\partial \operatorname{Ln} Z}{\partial \operatorname{Ln} P}\right)_{T}
$$

Figure 2.4 shows the $\mathrm{P}-\mathrm{h}$ diagram of $\mathrm{CO}_{2}$ with isolines of $z$ and $f_{T}$. The logarithmic factor of isothermal expansion $f_{T}$ has positive and negative values, and the line of $f_{T}=0$ separates these two regions. The points where $f_{T}$ is null indicate the pressure where the minimum compressibility factor is found for a specific temperature. Thus, the line of $f_{T}=0$ will be very interesting to find the lowest values of $z$ for positioning the compressor.

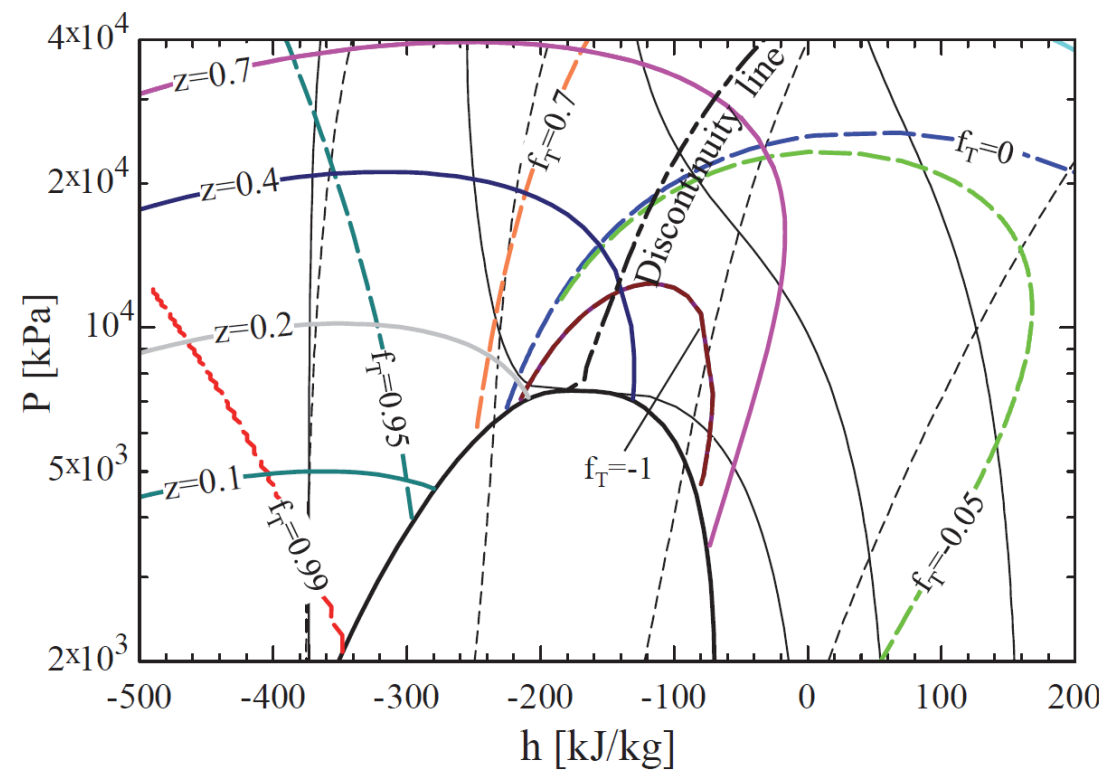

Figure 2.4. P-h diagram of $\mathrm{CO}_{2}$ around the critical point showing isolines of compressibility factor, $z$, and logarithmic factor of isothermal expansion, $f_{T}$.

For a specific pressure, the lower the temperature (and so the enthalpy) the lower the compressibility factor is, until a threshold, where the compressibility factor remains almost constant. Figure 2.4 shows that this threshold occurs to the left of the discontinuity band, where the curves of constant $z$ are almost horizontal. This means that the compressibility factor depends mainly on the pressure. This dependence is represented in Equation 2.5, where $\overline{f_{T}}$ is an integrated mean value of $f_{T}$, which is almost constant in the region to the left of the discontinuity band. 


$$
\frac{z}{z_{\text {ref }}}=\left(\frac{P}{P_{\text {ref }}}\right)^{\overline{f_{T}}}
$$

The reference point $r e f$ can be any point. For example, if a point with $z_{r e f}=1$ is chosen as reference point then the value of $z$ depends only on the pressure $P$, the reference pressure $P_{\text {ref }}$, and $\overline{f_{T}}$, which is an exponent close to 0.9 in the region to the left of the bending band. In this case, $P_{\text {ref }}$ would be a reference pressure (much higher than the critical pressure) without any role in the study of the cycle features. Note that these reference values will disappear in the cycle analysis as they appear on the two pressure levels of the cycle, the upper and lower ones. The relevant factor is the exponent $\overline{f_{T}}$, which must be determined with actual values of the equation of state.

The logarithmic factors of isothermal expansion and isobaric expansion, $f_{T}$ and $f_{P}$, will appear during the analysis of the relevant thermodynamic properties of cycle phases. The following sections focus on the characterization of compression and expansion through the analysis of the isentropic evolutions of temperature and volume.

\subsubsection{Isentropic evolution of temperature}

This section shows the process to obtain the thermodynamic property that characterizes the isentropic evolution of temperature. This process starts with the general Equation 2.6.

$$
\left(\frac{d u}{d T}\right)_{v}=\left(\frac{d h}{d T}\right)_{v}-\left(\frac{d P v}{d T}\right)_{v}
$$

The last term can be further developed by deriving Equation 1.8, which results in:

$$
\left(\frac{d P}{d T}\right)_{v}=\left(\frac{\partial z}{\partial T}\right)_{v} \frac{R T}{v}+z \frac{R}{v}
$$

And therefore:

$$
\left(\frac{d P v}{d T}\right)_{v}=R\left[T\left(\frac{\partial z}{\partial T}\right)_{v}+z\right]
$$

Substituting the last term from Equation 2.6 by Equation 2.8 leads to a general relation between the specific heats $c_{p}$ and $c_{v}$ :

$$
c_{v}=c_{P}-\alpha R T\left[T\left(\frac{\partial z}{\partial T}\right)_{v}+z\right]
$$

Equation 2.9 can be rearranged as: 


$$
c_{v}=c_{P}-R\left[\frac{T}{z}\left(\frac{\partial z}{\partial T}\right)_{P}+1\right]\left[\frac{T}{z}\left(\frac{\partial z}{\partial T}\right)_{v}+1\right]
$$

Thus, the adiabatic coefficient $\gamma$ can be expressed as:

$$
\gamma=1+\frac{R}{c_{v}}\left[\frac{T}{z}\left(\frac{\partial z}{\partial T}\right)_{P}+1\right]\left[\frac{T}{z}\left(\frac{\partial z}{\partial T}\right)_{v}+1\right]
$$

It is worth pointing out that the general evolution of pressure $P$ and temperature $T$ along an isentropic line cannot be expressed in terms of the adiabatic coefficient $\gamma$, and this is particularly true for the pericritical region. The exact evolution of $P$ and $T$ along an isentropic line can be found with the help of the following Maxwell equation:

$$
\left(\frac{\partial T}{\partial P}\right)_{S}=\left(\frac{\partial v}{\partial s}\right)_{P}
$$

The last term of Equation 2.12 can be expressed as:

$$
\left(\frac{\partial v}{\partial s}\right)_{P}=\frac{\left(\frac{\partial v}{\partial T}\right)_{P}}{\left(\frac{\partial s}{\partial T}\right)_{P}}
$$

The denominator is:

$$
\left(\frac{\partial s}{\partial T}\right)_{P}=\frac{1}{T}\left(\frac{\partial h}{\partial T}\right)_{P}=\frac{c_{P}}{T}
$$

And the numerator is obtained by deriving Equation 1.8:

$$
\left(\frac{\partial v}{\partial T}\right)_{P}=\frac{R}{P}\left[\left(\frac{\partial z}{\partial T}\right)_{P} T+z\right]
$$

Thus, the following relation is obtained from Equations 2.12-2.15:

$$
\left(\frac{\partial T}{\partial P}\right)_{S}=\left(\frac{\partial v}{\partial s}\right)_{P}=\frac{\frac{R}{P}\left[\left(\frac{\partial z}{\partial T}\right)_{P} T+z\right]}{\frac{C_{P}}{T}}
$$

Equation 2.16 can be rearranged as

$$
\frac{P}{T}\left(\frac{\partial T}{\partial P}\right)_{S}=\left(\frac{\partial \operatorname{LnT}}{\partial \operatorname{Ln} P}\right)_{S}=z \frac{R}{c_{P}}\left[\left(\frac{\partial \operatorname{Ln} Z}{\partial \operatorname{Ln} T}\right)_{P}+1\right]
$$

The isentropic evolution of the temperature is then given by a coefficient $g$, defined as 


$$
g=z \frac{R}{c_{P}}\left[f_{P}+1\right]
$$

The coefficient $g$ is rather small to the left of the critical temperature, because the logarithmic derivative $f_{P}$ is small, $z$ is much smaller than 1 , and $R$ is much lower than $c_{p}$, especially close to the critical point. This means that compressing the fluid will convey a very small increase of temperature. Figure 2.5 depicts relevant information on the evolution of the isentropic coefficient $g$.

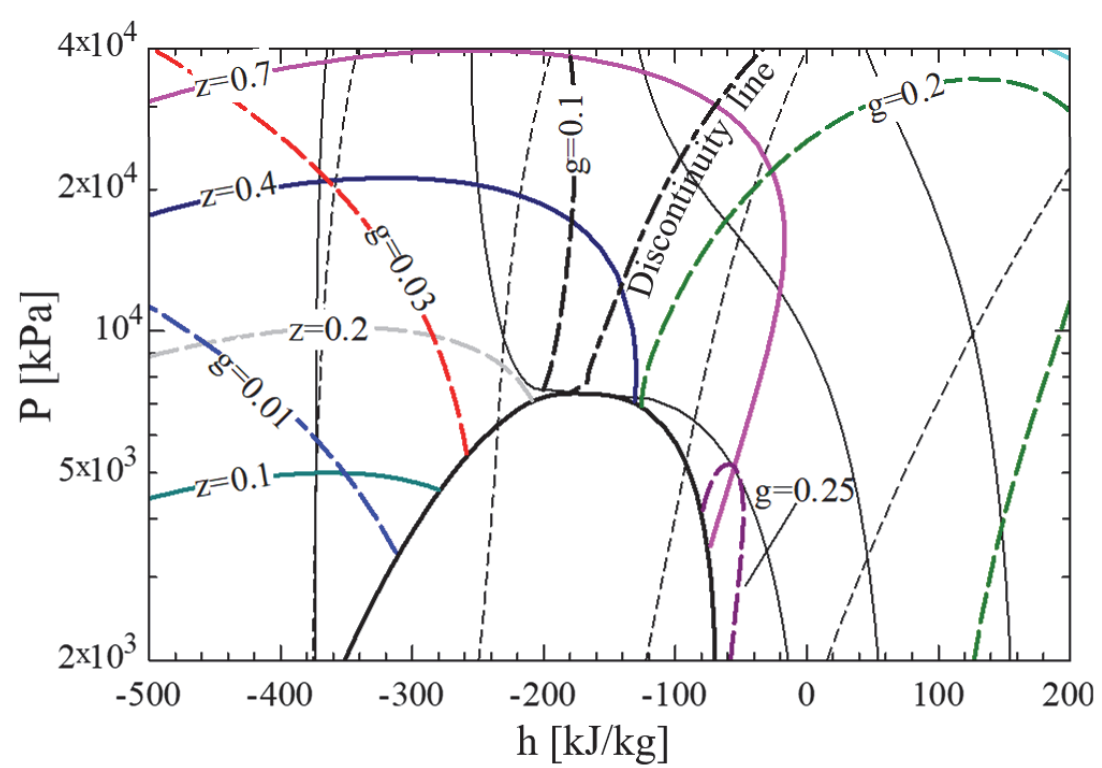

Figure 2.5. P-h diagram of $\mathrm{CO}_{2}$ around the critical point showing isolines of compressibility factor, $z$, and isentropic evolution of temperature coefficient, $g$.

The coefficient $g$ is a thermodynamic coefficient that relates temperature and pressure along an isentropic line. Equation 2.19 expresses this relation in an isentropic line starting at a point $\left(P_{0}, T_{0}\right)$ and finishing at $\left(P_{1}, T_{1}\right)$, where $\bar{g}$ is the integrated mean value of $g$ along the isentropic line.

$$
\frac{T_{1}}{T_{0}}=\left(\frac{P_{1}}{P_{0}}\right)^{\bar{g}}
$$

For example, in a compressor with $\mathrm{CO}_{2}$, reduced inlet temperature $T_{i n, r}=0.95\left(T_{r}=T / T_{c r}\right)$ and reduced inlet pressure $P_{i n, r}=1\left(P_{r}=P / P_{c r}\right)$, if the pressure ratio were $r=3$ then the temperature ratio from Equation 2.19 would be:

$$
\frac{T_{\text {out }, r}}{0.95}=3^{0.04}=1.045
$$

where the exponent $\bar{g}=0.04$ is obtained with the equation of state of $\mathrm{CO}_{2}$. 
The resulting reduced compressor outlet temperature is $T_{o u t, r}=0.99$, which implies less than $15 \mathrm{~K}$ over the inlet temperature. If the compression started at a reduced temperature $T_{i n, r}=1.05$ then the final temperature would be almost $100 \mathrm{~K}$ over the starting point. Friction forces inside the compressor will heat the fluid additionally, but they will be proportional to the specific work of the compressor. Thus, the lower the temperature increment during the compression phase, the smaller the additional heating will be (as the isentropic efficiency of the machine will remain almost invariant).

Note that under the assumption that the compressibility factor $z$ only depends on pressure, $f_{p}$ is zero, and then the value of the isentropic evolution of temperature coefficient, $g$, is:

$$
g=z \frac{R}{c_{P}}
$$

This is also true for the case of $z=1$, which corresponds to the ideal gas equation of state, where $g$ would be:

$$
g=\frac{R}{c_{P}}=\frac{c_{P}-c_{v}}{c_{P}}=\frac{\gamma-1}{\gamma}
$$

\subsubsection{Isentropic evolution of volume}

This section shows the process to obtain the thermodynamic property that characterizes the isentropic evolution of the specific volume. This process begins with the general principle from Equation 2.23.

$$
d s=c_{v} \frac{d T}{T}+\left(\frac{\partial P}{\partial T}\right)_{v} d v
$$

Thus, the relation between volume $v$ and temperature $T$ along an isentropic line $(d S=0)$ is:

$$
c_{v} \frac{d T}{T}=-\left(\frac{\partial P}{\partial T}\right)_{v} d v
$$

In order to develop the last term, the triple product rule gives the following relation:

$$
\left(\frac{\partial P}{\partial T}\right)_{V}=-\frac{\left(\frac{\partial V}{\partial T}\right)_{P}}{\left(\frac{\partial V}{\partial P}\right)_{T}}
$$

Numerator and denominator can be calculated by deriving Equation 1.8: 


$$
\begin{aligned}
& \left(\frac{\partial V}{\partial T}\right)_{P}=\frac{z R}{P}\left[f_{P}+1\right] \\
& \left(\frac{\partial V}{\partial P}\right)_{T}=\frac{V}{P}\left[f_{T}-1\right]
\end{aligned}
$$

Thus, combining Equations 2.24-2.27:

$$
c_{v} \frac{d T}{T}=z R \frac{f_{P}+1}{f_{T}-1} \frac{d v}{v}
$$

From Equation 2.28 and 2.18, the relation between specific volume $v$ and pressure $P$ along the isentropic line can expressed as

$$
\frac{d T}{T}=\frac{z R}{c_{V}} \frac{f_{P}+1}{f_{T}-1} \frac{d v}{v}=\frac{z R\left(f_{P}+1\right)}{c_{P}} \frac{d P}{P}
$$

And therefore:

$$
\frac{d v}{v}=\left(f_{T}-1\right) \frac{c_{V}}{c_{P}} \frac{d P}{P}
$$

The isentropic evolution of the volume is then given by a coefficient $m$, defined as

$$
m=\frac{\left(1-f_{T}\right) c_{V}}{c_{P}}
$$

In an isentropic line with an integrated mean coefficient $\bar{m}$ starting at a point $\left(P_{0}, T_{0}\right)$ and finishing at $\left(P_{1}, T_{1}\right)$, the relation between pressure $P$ and specific volume $v$ is:

$$
\frac{v_{1}}{v_{0}}=\left(\frac{P_{1}}{P_{0}}\right)^{-\bar{m}}
$$

When the thermodynamic evolution is placed in a domain where $f_{T}$ is close to 1 (but lower than 1), $m$ is rather small, which implies low compression work. On the contrary, for the case of $z=1$ (ideal gas) the value of $f_{T}$ is zero, and $m$ is $1 / \gamma$. In between the limits of $f_{T} \approx 1$ and $z \approx 1$, there is a region where $f_{T}=0$, and the ratio $c_{p} / c_{v}$ determines the value of $m$ in this region.

In summary, the lower the isentropic coefficients $g$ and $m$, the better the compressor behavior will be. Reducing these two coefficients can be achieved by reducing the compressor inlet enthalpy. However, if the whole cycle is moved towards lower enthalpies then the turbine work decreases. The balance between reducing the turbine specific work and reducing the 
compressor work, together with other specific features of thermodynamic cycles working around the critical point, will be analysed in the following section.

\subsection{The ideal pericritical cycle}

The pericritical cycle will be characterized in this section according to the compressor position in the thermodynamic diagram. This characterization attempts to abstract from the fluid as much as possible by using reduced variables, mainly pressure $\left(P_{r}=P / P_{c r}\right)$ and temperature $\left(T_{r}=T / T_{c r}\right)$. Reduced enthalpy and reduced entropy will refer to the dimensionless variables $h_{r}=h / R T_{c r}$ and $s_{r}=S / R$, respectively. The equation of state of $\mathrm{CO}_{2}$ is used to carry out the calculations, but the variables are expressed in its reduced form. In this way, the major trends identifying the features of each thermodynamic region can be extrapolated to fluids able to work in that region.

The cycles of the analysis will have compressor inlet temperatures between $T_{c, i n, r}=0.95$ and $T_{c, i n, r}=1.05$, and a Carnot factor $\tau=3\left(\tau=T_{t, i n} / T_{c, i n}\right)$. All the cycles will have the same Carnot efficiency (defined in Equation 1.10) as they have the same Carnot factor. In this way, all cycles will have the same maximum theoretical efficiency, and the compressor position will be the parameter determining the real efficiency.

Figure 2.6 presents an example of pericritical cycle in P-h and T-s diagrams. The high-side pressure is above the critical pressure and the low-side pressure is below. The reduced compressor inlet temperature is $T_{c, i n, r}=0.95$. The compressor works to the left and above the discontinuity line (DL) in both diagrams, which means that it works in the liquid region. High- and low-pressure sides cross the discontinuity line and, therefore, the thermodynamic properties will experience strong variations along this crossing, especially in the low-pressure side. As the low-pressure side is below the critical pressure, the discontinuity line occupies a certain range of enthalpies and entropies during the cooling, and the temperature is constant in this range.

The discontinuity line grows rapidly in pressure, but slowly in temperature. This means that large pressure variations in the discontinuity line are associated to small temperature variations. The maximum temperature drawn in the discontinuity line of Figure 2.6 is $T_{r}=1.45$, which is associated to a pressure $P_{r}=6.1$. The discontinuity line disappears at higher pressures (and so at higher temperatures) because the property variation along the isobar is almost null. 

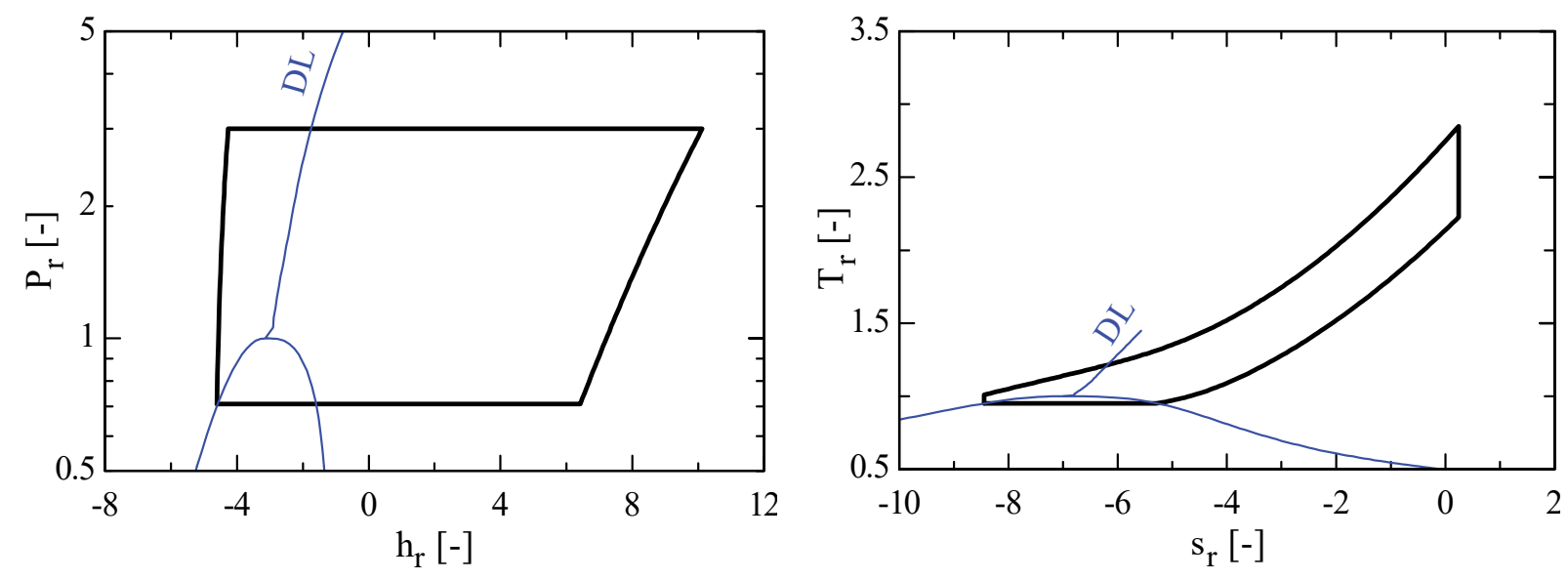

Figure 2.6. Pericritical cycle working partially above the critical pressure in diagrams P-h (left) and T-s (right)

Figure 2.7 shows another example of pericritical cycle, in this case working completely above the critical pressure. The main changes with respect to Figure 2.6 are that the reduced compressor inlet temperature is now $T_{c, i n, r}=1.05$ and the low-side pressure is above the critical pressure. The rest of the conditions are similar. The low-side pressure is still to the left (and therefore above) the discontinuity line in both diagrams. Thus, the thermodynamic properties along the cooling also experience strong variations. In this case, the temperature during part of the cooling is not constant, but its variation is very small.
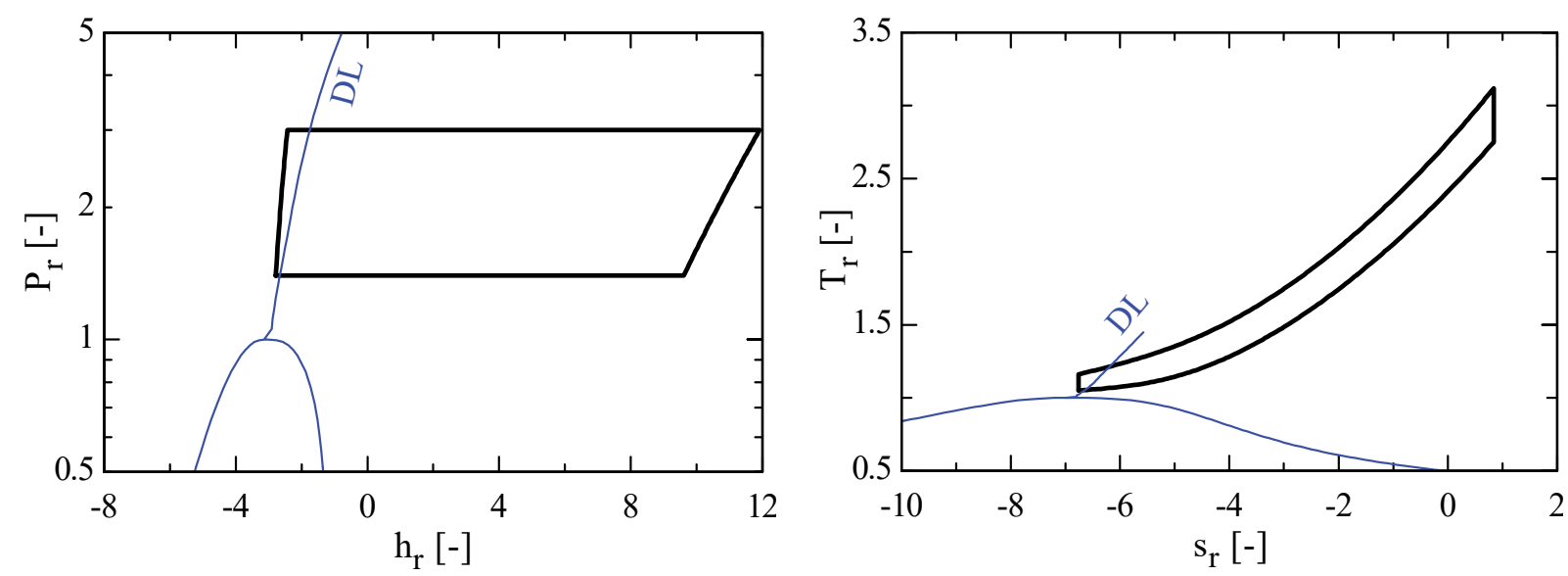

Figure 2.7. Pericritical cycle working above the critical pressure in diagrams $\mathrm{P}-\mathrm{h}$ (left) and T-s (right)

Figure 2.6 and Figure 2.7 show two pericritical cycles with the compressor working above the discontinuity line. However, pericritical cycles also include those with the compressor working below this line. The purpose of Figure 2.6 and Figure 2.7 is to show that two cycles that are normally studied separately as transcritical and supercritical cycles (Kim, Kim and 
Favrat, 2012) present similar characteristics, which means that they may be studied under the same analysis. Pericritical cycles agglutinate all the cycles working around the critical point.

\subsubsection{Closing conditions}

The thermodynamic properties studied in previous section appear in the different phases of the cycle. Each phase will be ruled by different parameters, and all the phases will depend on each other due to the closed conditions of the cycle. The purpose of this section is to link the thermodynamic properties of the different phases in a single equation.

The first step to close the cycle conditions is to analyse the evolution along an isobar. It is particularly important to characterize the increase in temperature with respect to the increase in specific volume, which is the relevant parameter for the mechanical work in the turbine. The derivative of Equation 1.8 (definition of compressibility factor) along an isobar results in the following equation:

$$
\frac{d z}{Z}+\frac{d T}{T}-\frac{d v}{v}=0
$$

If the definition of the logarithmic factor of isobaric expansion, $f_{P}$, is introduced in Equation 2.33 then:

$$
\left(\frac{\frac{\partial v}{v}}{\frac{\partial T}{T}}\right)_{P}=f_{P}+1
$$

And dividing Equation 2.34 by the specific heat $c_{p}$ :

$$
\left(\frac{\frac{\partial v}{v}}{\partial s}\right)_{P}=\frac{f_{P}+1}{c_{P}}
$$

The integration of Equation 2.35 along the high- and low-side pressures, $P_{H}$ and $P_{L}$, results in Equations 2.36 and 2.37, where the subscripts $c$ and $t$ stand for compressor and turbine, and in and out for inlet and outlet.

$$
\begin{aligned}
& \ln \frac{v_{t, \text { in }}}{v_{c, \text { out }}}=\Delta s\left(\frac{f_{P}+1}{c_{P}}\right)_{P_{H}} \\
& \ln \frac{v_{t, \text { out }}}{v_{c, \text { in }}}=\Delta s\left(\frac{f_{P}+1}{c_{P}}\right)_{P_{L}}
\end{aligned}
$$


Note that the entropy variation $\Delta s$ is the same in both isobars. Thus, equations 2.36 and 2.37 can be combined in the following relation:

$$
\ln \left(\frac{v_{t, \text { in }}}{v_{c, \text { out }}} \frac{v_{c, \text { in }}}{v_{t, \text { out }}}\right)=\Delta S\left[\left(\frac{f_{P}+1}{c_{P}}\right)_{P_{H}}-\left(\frac{f_{P}+1}{c_{P}}\right)_{P_{L}}\right]
$$

The relations between the volumes at the beginning and at the end of an isentropic line have been defined in Equations 2.19 and 2.32. For the compressor, the relation would be:

$$
\frac{v_{c, \text { out }}}{v_{c, \text { in }}}=r^{-m_{c}}
$$

And for the turbine:

$$
\frac{v_{t, \text { in }}}{v_{t, \text { out }}}=\frac{z_{t, \text { in }}}{z_{t, \text { out }}} r^{g_{t}-1}
$$

When the turbine works close to ideal conditions, the values of the compressibility factor $z$ t,in and $z_{t, \text { out }}$ will be both close to 1 , and $g_{t}$ is very close to $(\gamma-1) / \gamma$. Thus, Equation 2.40 can be expressed as:

$$
\frac{v_{t, \text { in }}}{v_{t, \text { out }}}=r^{-1 / \gamma}
$$

Combining Equations 2.38-2.41, the cycle is closed with the following relation:

$$
\ln r=\frac{\Delta s\left[\left(\frac{f_{P}+1}{c_{P}}\right)_{P_{H}}-\left(\frac{f_{P}+1}{c_{P}}\right)_{P_{L}}\right]}{m_{c}-\frac{1}{\gamma}}
$$

The closing relation of the cycle can also be written in terms of temperature limits. The relation at the ends of the isobar between the temperature and the specific volumes can be obtained from Equation 2.34, and expressed as:

$$
\ln \frac{v_{t}}{v_{c}}=\overline{\left(f_{p}+1\right)_{P}} \ln \frac{T_{t}}{T_{c}}
$$

where $\overline{\left(f_{p}+1\right)}$ is an average in logarithmic scale defined in Equation 2.44.

$$
\overline{\left(f_{p}+1\right)_{P}}=\frac{\int_{c}^{t}\left(f_{p}+1\right) \frac{d T}{T}}{\int_{c}^{t} \frac{d T}{T}}
$$


Thus, the relation between temperature and specific volume in the high- and the low side pressures can be expressed as:

$$
\begin{aligned}
\left(\frac{v_{t, \text { in }}}{v_{c, \text { out }}}\right) & =\left(\frac{T_{t, \text { in }}}{T_{c, \text { out }}}\right)^{\overline{\left(f_{p}+1\right)}} P_{H} \\
\left(\frac{v_{t, \text { out }}}{v_{c, \text { in }}}\right) & =\left(\frac{T_{t, \text { out }}}{T_{c, \text { in }}}\right)^{\overline{\left(f_{p}+1\right)} P_{L}}
\end{aligned}
$$

where the ends are characterized by an isentropic line with the following equations:

$$
\begin{aligned}
\left(\frac{v_{c, \text { out }}}{v_{c, \text { in }}}\right) & =\left(\frac{T_{c, \text { out }}}{T_{c, \text { in }}}\right)^{-m_{c} / g_{c}} \\
\left(\frac{v_{t, \text { in }}}{v_{t, \text { out }}}\right) & =\left(\frac{T_{t, \text { in }}}{T_{t, \text { out }}}\right)^{-1 /(\gamma-1)}
\end{aligned}
$$

The cycle is closed by combining Equations 2.43-2.48 in the following relation:

$$
\ln \frac{T_{t, \text { in }}}{T_{c, \text { in }}}=\frac{g_{c}\left(\overline{\left(f_{p}+1\right)_{P_{H}}}+\frac{m_{c}}{g_{c}}\right)-\left(\overline{\left(f_{p}+1\right)_{P_{L}}}+\frac{1}{\gamma-1}\right)\left(\frac{\gamma-1}{\gamma}\right)}{\left(f_{p}+1\right)_{P_{H}}-\overline{\left(f_{p}+1\right)_{P_{L}}}} \ln r
$$

Equation 2.49 is a condition to be fulfilled by the compression ratio $r$ and the limiting temperatures ratio $T_{t, \text { in }} / T_{c, \text { in }}$ (known as Carnot number), according to the lumped parameters of the four phases of the cycle, which depend on the equation of state of the working fluid. The following sections analyse the relation between these parameters and the cycle behavior.

\subsubsection{Specific work}

The turbine inlet marks the point of highest enthalpy, with a specific volume $v_{t, \text { in }}$ and a pressure $P_{t, i n}$. The isentropic evolution of the specific volume from Equation 2.32 can be rewritten as:

$$
\frac{v_{t, i n}}{v}=\left(\frac{P_{t, i n}}{P}\right)^{-m_{t}}
$$

Thus, the specific work delivered by the turbine (Equation 1.4) can be formulated as:

$$
w_{t}=\frac{P_{t, i n} v_{t, i n}}{1-m_{t}}\left(1-r^{m_{t}-1}\right)=\frac{z_{t, i n} R T_{t, i n}}{1-m_{t}}\left(1-r^{m_{t}-1}\right)
$$


If the turbine worked in the ideal gas region, the equation could be simplified to

$$
w_{t}=\frac{R T_{t, i n}}{j}\left(1-r^{-j}\right)
$$

where $j$ stands for $(\gamma-1) / \gamma$.

The compressor work can be formulated in a similar way. If the compressor inlet is defined by a point with volume $v_{c, i n}$ and pressure $P_{c, i n}$, the isentropic evolution of the specific volume along the compression is:

$$
\frac{v}{v_{c, \text { in }}}=\left(\frac{P}{P_{c, \text { in }}}\right)^{-m_{c}}
$$

And therefore the compressor specific work $w_{c}$ can be expressed as:

$$
w_{c}=\frac{P_{c, i n} v_{c, i n}}{1-m_{c}}\left(r^{1-m_{c}}-1\right)=\frac{z_{c, i n} R T_{c, i n}}{1-m_{c}}\left(r^{1-m_{c}}-1\right)
$$

If the compressor worked in the range where $f_{T}$ is close to 1 (and therefore $\mathrm{m}<<1$ ) then Equation 2.54 may be simplified to

$$
w_{c}=z_{c, i n} R T_{c, i n}(r-1)
$$

An important parameter to characterize the cycle performance is the ratio $w_{c} / w t$. From Equations 2.51 and 2.53, this parameter can be written as

$$
\frac{w_{c}}{w_{t}}=\frac{z_{c, i n} T_{c, i n}}{z_{t, \text { in }} T_{t, i n}} \cdot \frac{1-m_{t}}{1-m_{c}} \cdot \frac{r^{1-m_{c}}-1}{1-r^{m_{t}-1}}
$$

The purpose of this chapter will be to analyse the influence of the compressor in pericritical cycles. In order to set aside the influence of the turbine as much as possible, the calculations will be made for high temperatures, which means that the turbine works close to ideal conditions, where the heat capacity ratio $\gamma$ is more or less constant. In this case, Equation 2.56 could be simplified to:

$$
\frac{w_{c}}{w_{t}}=\frac{z_{c, i n} T_{c, i n}}{T_{t, i n}} \cdot \frac{j}{1-m_{c}} \cdot \frac{r^{1-m_{c}}-1}{1-r^{-j}}
$$

Note that if the compressor also worked at ideal conditions, Equation 2.56 could be further simplified to:

$$
\frac{w_{c}}{w_{t}}=\frac{T_{c, i n}}{T_{t, i n}} \cdot r^{j}=\tau \cdot r^{\frac{\gamma-1}{\gamma}}
$$


Two pericritical cycles will be analysed in order to study the parameter $w_{c} / w_{t}$. These cycles have both a low-side pressure $P_{L, r}=1$ and a Carnot factor $\tau=3$. The difference is the compressor inlet reduced temperature: $T_{c, i n, r}=0.95$ and $T_{c, i n, r}=1.05$. Figure 2.8 shows a scheme of these two cycles in the case of a pressure ratio $r=3$. The Carnot factor $\tau=3$ will be maintained in all the calculations of this chapter in order to set the high turbine inlet temperature in the ideal gas region.

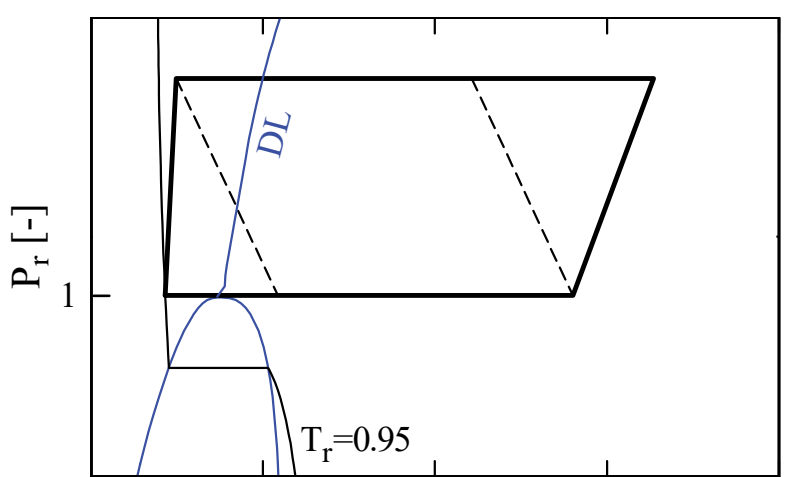

$\mathrm{h}_{\mathrm{r}}[-]$

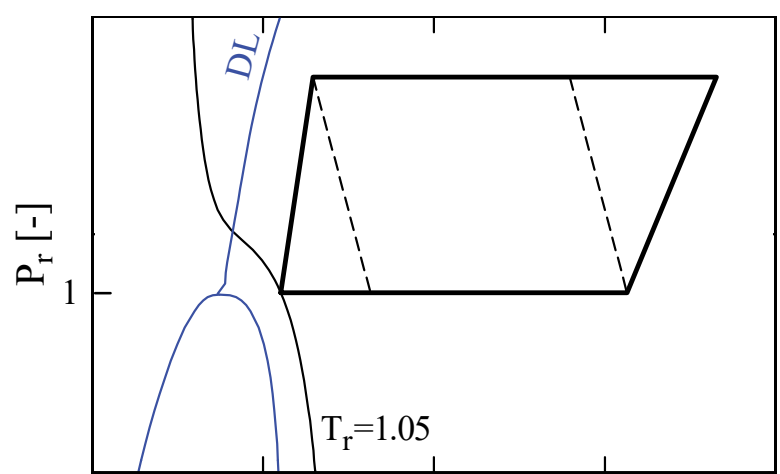

$\mathrm{h}_{\mathrm{r}}[-]$

Figure 2.8. Pericritical cycles with compressor inlet reduced temperature $T_{c, i n, r}=0.95$ (left) and $T_{c, i n, r}=1.05$ (right)

In the cycles of Figure 2.8, both turbines work close to ideal gas conditions. Thus, Equation 2.57 will define the parameter $w_{c} / w_{t}$. The only variables in this equation will be the pressure ratio $r$ and the parameter $m_{c}$; the rest of the parameters are defined by compressor inlet and turbine inlet conditions.

Figure 2.9 shows the evolution of the parameter $w_{c} / w_{t}$ as a function of the compression ratio $r$ for the cases with a compressor inlet reduced temperature $T_{c, i n, r}=0.95$ and $T_{c, i n, r}=1.05$. When the temperature is $T_{c, i n, r}=0.95$, the $w_{c} / w_{t}$ values are much lower due to the lower compressibility factor at the compressor inlet $\left(z_{c, i n}=0.15\right.$ at $T_{c, i n, r}=0.95$ and $z_{c, \text { in }}=0.6$ at $\left.T_{c, i n, r}=1.05\right)$. In fact, the ratio of $w_{c} / w_{t}$ is equal to the ratio of compressibility factors at low pressure ratios:

$$
\frac{\left(w_{c} / w_{t}\right)_{T_{c, i n, r}=1.05}}{\left(w_{c} / w_{t}\right)_{T_{c, i n, r}=0.95}}=\frac{\left(z_{c, i n}\right)_{T_{c, i n, r}=1.05}}{\left(z_{c, i n}\right)_{T_{c, i n, r}=0.95}}
$$

Thus, for pressure ratio $r=1$ the $w_{c} / w_{t}$ value at temperature $T_{c, i n, r}=1.05$ is 3.8 times greater than $w_{c} / w_{t}$ at $T_{c, i n, r}=0.95$. The different values of $m_{c}$ lowers this difference when the pressure ratio increases, but the difference is still large. For example, at pressure ratio $r=5, w_{c} / w_{t}$ at temperature $T_{c, i n, r}=1.05$ is 2.3 times greater. 


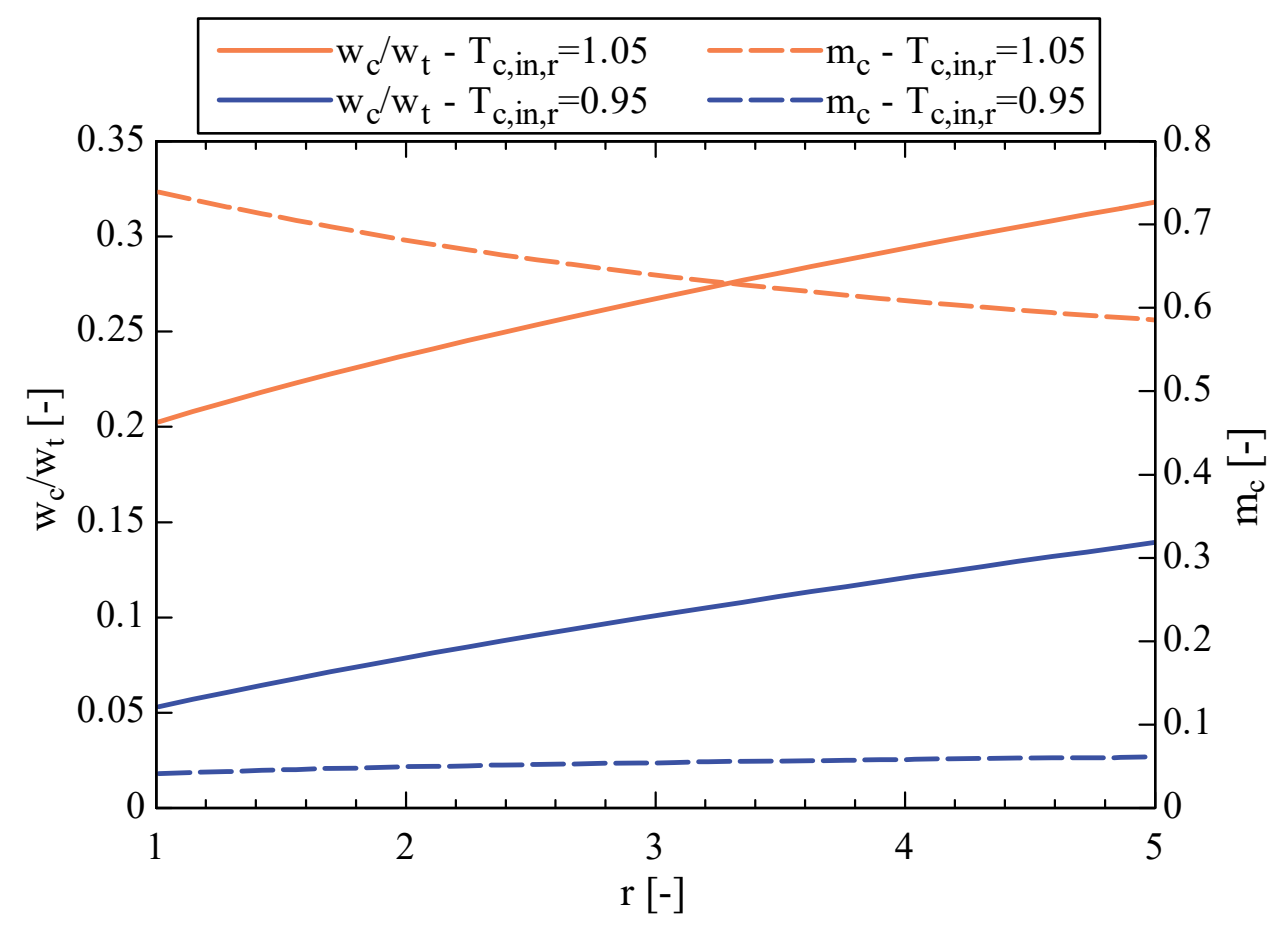

Figure 2.9. Parameter $w_{c} / w_{t}$ and coefficient $m_{c}$ as a function of the pressure ratio, $r$, in a pericritical cycle with compressor inlet temperatures $T_{c, i n, r}=0.95$ and $T_{c, i n, r}=1.05$.

The compressibility factor value rules the behavior of the parameter $w_{c} / w_{t}$, and the discontinuity line rules the compressibility factor behavior. This means that the discontinuity line separates not only two regions with different compressibility factor values, but also with different compression works. The region to the left of the discontinuity line has very good features for placing the point of minimum enthalpy of the cycle. The low value of the compressibility factor in this point is very important to keep low values of $w_{c} / w_{t}$, even when the compression ratio is large.

The parameter $w_{c} / w_{t}$ must be small for having a high efficiency. The smaller this parameter, the higher the efficiency is. If the regeneration between the compressor outlet and turbine outlet temperatures were ideal, the optimum pressure ratio would be the minimum possible $(r=1)$ to minimize $w_{c} / w_{t}$. However, next section will show that the regeneration is not ideal and that the low values of $w_{c} / w_{t}$ will be counteracted by large limitations in the regeneration.

\subsubsection{Limitations in the regeneration}

An ideal balanced heat exchanger has the same heat capacity in both fluids, hot and cold:

$$
\left(\dot{m} c_{P}\right)_{h o t}=\left(\dot{m} c_{P}\right)_{c o l d}
$$

In a balanced heat exchanger, the temperature lines of hot and cold fluids are parallel, and an adequate heat transfer at uniform rate happens along the exchanger. Such an ideal situation is 
not possible in the regeneration of pericritical cycles because, although the mass flow rate $\dot{m}$ is the same in both sides of the heat exchanger, the specific heat $c_{p}$ is not. The specific heat highly depends on pressure and temperature. Figure 2.10 shows the specific heat variation of $\mathrm{CO}_{2}$ as a function of temperature at different pressures above and below the critical pressure. The specific heat has a different maximum value depending on the pressure. These points of maximum specific heat define the pseudocritical line (very similar to the discontinuity line for pressures below $10 \mathrm{MPa}$ ), previously defined and shown in Figure 2.2. For pressures above the critical pressure $\left(P_{c r}=7.4 \mathrm{MPa}\right)$, the closer to the critical temperature $\left(T_{c r}=31{ }^{\circ} \mathrm{C}\right)$ the sharper the variations of specific heat become. For pressures equal to or lower than the critical pressure, the variation is abrupt and the specific heat tends to infinite.

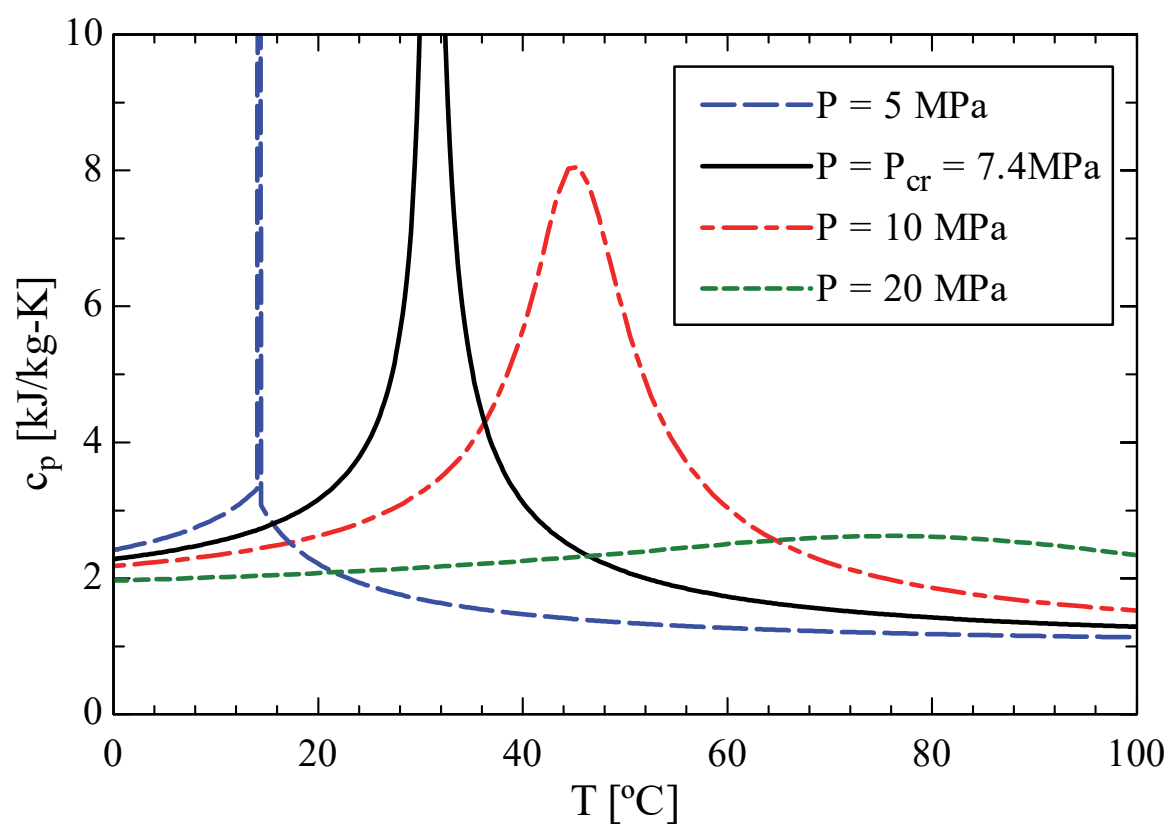

Figure 2.10. Specific heat, $c_{p}$, of $\mathrm{CO}_{2}$ as a function of the temperature, $T$, for different pressures below and above the critical pressure $\left(P_{c r}\right)$

Another important feature in the heat regeneration is related to the path of the isothermal lines in the thermodynamic P-h diagram. Isothermal lines in a real gas close to the critical point are deflected to lower enthalpy values when the pressure increases. Equation 2.61 expresses the relation between the enthalpy variation along an isothermal line and the pressure variation.

$$
d h_{T}=-f_{P} v_{T} d P
$$

The total enthalpy variation when the pressure increases depends on the integration paths along the isothermal, and the main differences among them come from the logarithmic factor of isobaric expansion, $f_{p}$. 
Figure 2.11 shows a schematic diagram of a generic regenerative pericritical cycle limited by the points corresponding to compressor inlet, compressor outlet, turbine inlet and turbine outlet. The lines depicted inside the cycle correspond to linearized isothermals with different slopes $(d h / d P)_{T}$. The temperature $T_{m}$ has the minimum slope $(d h / d P)_{T}$ (and maximum in absolute value). This means that the $v f_{P}$ value will be maximum in this isothermal line (see Equation 2.61).

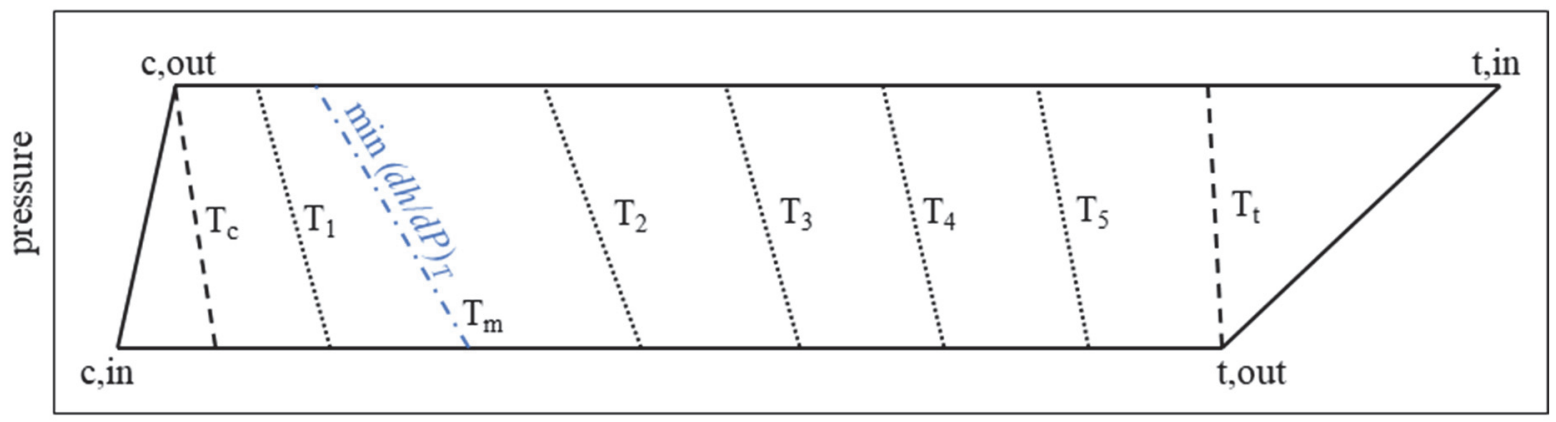

enthalpy

Figure 2.11. Schematic diagram of a generic pericritical regenerative cycle limited by the points corresponding to the compressor inlet (c,in), compressor outlet (c,out), turbine inlet $(\mathrm{t}, \mathrm{in})$ and turbine outlet $\left(\mathrm{t}\right.$, out). Some linearized isothermal lines $\mathrm{T}_{\mathrm{x}}$ are shown inside the cycle.

The value of the derivative $(d h / d P)_{T}$ depends on the range of pressures in which the cycle works. Figure 2.12 shows the value of the derivative $(d h / d P)_{T}$ as a function of the temperature in a cycle working between the critical pressure and a high-side pressure $P_{H, r}=3$. The value of the derivative $(d h / d P)_{T}$ depends on the logarithmic factor of isobaric expansion, $f_{P}$, along the isothermal $T$. Figure 2.12 shows that the low-pressure logarithmic factor of isobaric expansion, $f_{P, L P}$, has a high impact in the trend of $(d h / d P)_{T}$ due to the greater proximity of the low-pressure side to the critical point, and so the larger values of $f_{P}$.

When the fluid is cooled along the low-side pressure, the derivative $(d h / d P)_{T}$ decreases (increases the absolute value) due to the approximation to the critical point, and so the higher values of $f_{P}$. The minimum $(d h / d P)_{T}$ is achieved in between the maximum $f_{P}$ values of the low- and high-pressure sides, $f_{P, L P}$ and $f_{P, H P}$. The temperature $T_{m}$ is closer to the temperature corresponding to the discontinuity line (points with maximum $f_{P}$ ) in the low-pressure side due to the larger $f_{P}$ values. When the fluid is cooled further, the derivative goes again close to zero (as it was in the ideal gas region at high temperature). 


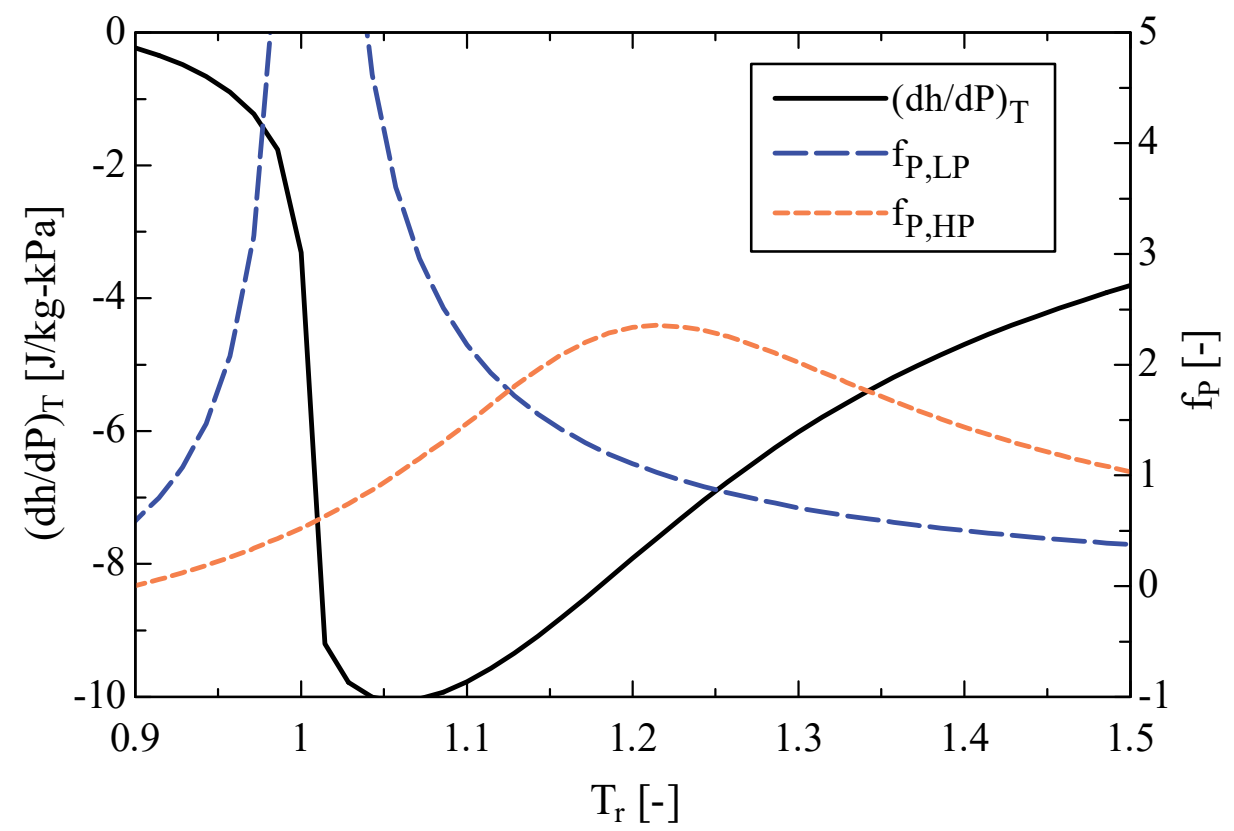

Figure 2.12. Values of the derivative $(d h / d P)_{T}$ and logarithmic factor of isobaric expansion in the low-pressure and high-pressure sides, $f_{P, L P}$ and $f_{P, H P}$, respectively, as a function of the temperature, $T$, in a cycle working between the critical pressure and a pressure $P_{r}=3$

Note that the variation of the logarithmic factor of isobaric expansion with the temperature in Figure 2.12 is similar to the variation of specific heat in Figure 2.10. The logarithmic factor has a different maximum value depending on the pressure, with stronger variations as the fluid get closer to the critical point. Moreover, the higher the pressure, the higher the temperature corresponding to the discontinuity line (points with maximum $f_{P}$ ) is. This means that the temperature $T_{m}$, in between the temperatures corresponding to the discontinuity line in the low- and high side pressures, depends on these pressures. The temperature $T_{m}$ will be higher in pericritical cycles with higher pressures, and vice versa.

The pericritical cycle in Figure 2.11 represents a generic regenerative pericritical. The lowpressure side may be below or above the critical pressure. The scheme of the isothermal lines represents both cases as they are in a linearized form. However, not all the pericritical cycles will have the minimum $(d h / d P)_{T}$ inside the cycle. Figure 2.13 shows a pericritical cycle with the isothermal line with minimum $(d h / d P)_{T}$ outside the cycle. If the compressor outlet temperature is greater than the temperature of the isothermal line with minimum $(d h / d P)_{T}\left(T_{m}\right.$ in Figure 2.11) then the isothermal line with minimum slope inside the cycle is the corresponding to the compressor outlet temperature $T_{c}$. It means, in this case, $T_{c}$ and $T_{m}$ are the same. 


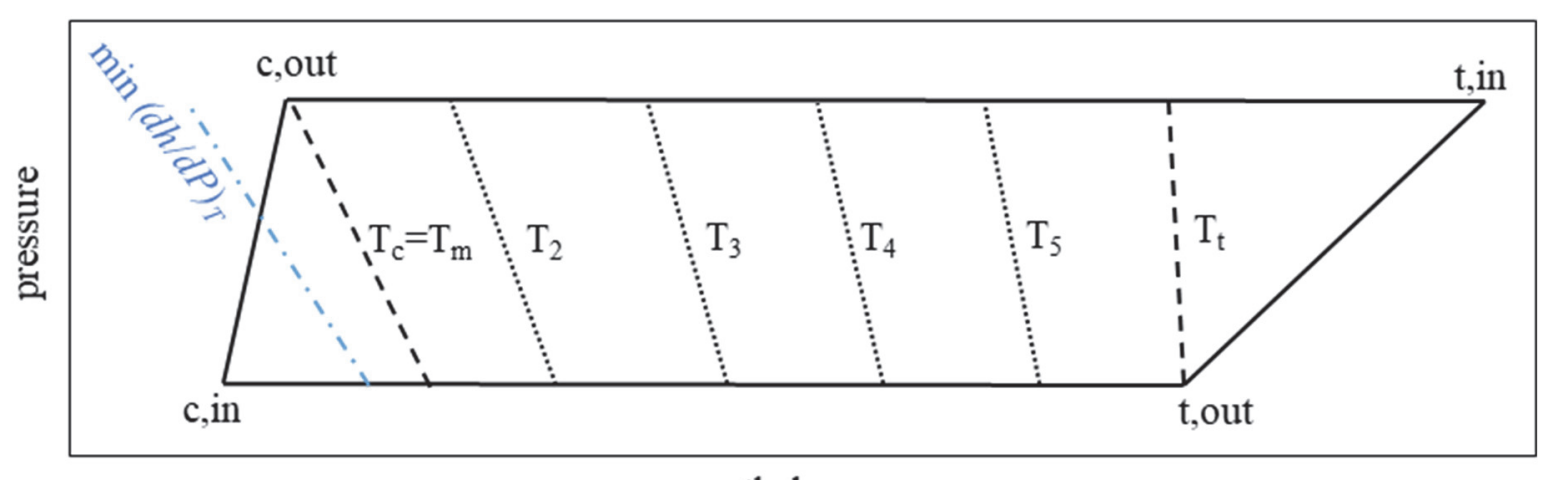

enthalpy

Figure 2.13. Schematic diagram of a pericritical regenerative cycle with the isothermal line with minimum $(d h / d P)_{T}$ outside the cycle.

Heat transfer is only possible from a fluid to another that is at lower temperature. At each section of the regenerator, the temperature of the low-pressure side must be greater than the temperature of the high-pressure side. Thus, the minimum temperature of the low-pressure side to heat the compressor outlet is $T_{c}$, and the maximum achievable temperature in the highpressure side from the low-pressure side is $T_{t}$. However, in a cycle such as the one shown in Figure 2.11, an enthalpy balance will prove that a temperature higher than $T_{c}$ will be needed to heat the compressor outlet and a temperature lower than $T_{t}$ will be the maximum achievable in the high-pressure side.

The regeneration phase can be interpreted as an internal virtual loop evolving inside a fourphase cycle made of two isothermal lines and two isobars. Figure 2.14 shows a differential part of the regeneration phase with two isobars (the lower one at $P$, the higher one at $P+d P$ ) and two isothermals (the cooler one at $T$, the hotter one at $T+d T$ ).

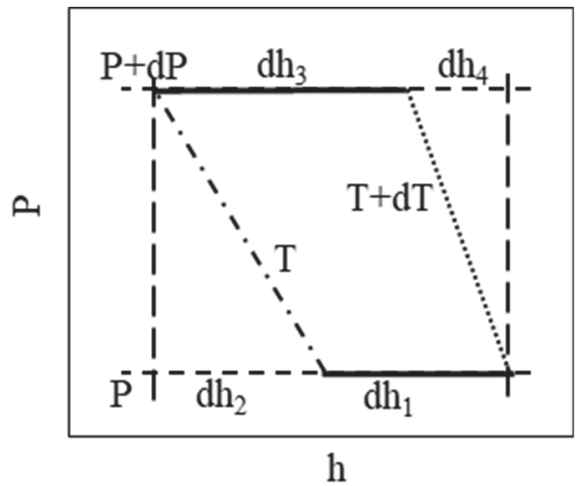

Figure 2.14. Enthalpy balance at differential scale in part of the cycle with two isothermal lines and two isobars.

The enthalpy balance of Figure 2.14 can be expressed as: 


$$
\begin{gathered}
d h_{1}+d h_{2}=d h_{3}+d h_{4} \\
d h_{1}=c_{P} d T \\
d h_{2}=(\alpha T-1) v_{T} d P=f_{P} v_{T} d P \\
d h_{3}=\left(c_{P}+\left(\frac{\partial c_{P}}{\partial P}\right)_{T} d P\right) d T \\
d h_{4}=\left(f_{P}+\left(\frac{\partial f_{P}}{\partial T}\right)_{P} d T\right)\left(v_{T}+\left(\frac{\partial v_{T}}{\partial T}\right)_{P} d T\right) d P
\end{gathered}
$$

The enthalpy differential $d h_{1}$ can only heat an enthalpy differential $d h_{1}$ in the high-side pressure $P+d P$. As the enthalpy differential $d h_{3}$ is greater than $d h_{1}, d h_{1}$ can heat only part of $d h_{3}$. Thus, the temperature $T+d T$ of the low-side pressure $P$ will heat a lower temperature (between $T$ and $T+d T$ ) in the high-side pressure $P+d P$. The reason of the difference between $d h_{1}$ and $d h_{3}$ is the difference in specific heat $\left(\partial c_{P} / \partial P\right)_{T}$.

Note that Figure 2.14 represents a differential part of the regeneration phase to the right of the isothermal line $T_{m}$ in Figure 2.11. However, the inclination of the isothermal lines in a differential part to the left of the isothermal line $T_{m}$ would be different: the line corresponding to $T$ would be more vertical than the one corresponding to $T+d T$. The enthalpy balance from Equations 2.62-2.66 would be similar. In this case, the enthalpy differential $d h_{1}$ will be greater than $d h_{3}$ due to the greater specific heat in the low-side pressure. Thus, the temperature $T$ of the high-side pressure $P+d P$ will be heated by a higher temperature (between $T$ and $T+d T$ ) in the low-side pressure $P$.

The result of applying the energy balance to the whole regeneration is shown in Figure 2.15a. The two behaviors explained can be appreciated to the left and to the right of the point with minimum temperature difference, commonly called pinch point, which corresponds with $T_{m}$. The specific heat of the low-pressure side to the left of $T_{m}$ is greater than the high-pressure one, and to the right is smaller. The specific heats of both isobars are equal in $T_{m}$.

Note that Figure 2.15a represents the case of a cycle with the minimum $(d h / d P)_{T}$ inside the cycle, such as in Figure 2.11. If the cycle had the minimum $(d h / d P)_{T}$ outside the cycle, such as in Figure 2.13, the specific heat of the low-pressure side would never be greater than the highpressure one. Thus, the minimum temperature difference is not located in the middle of the regeneration, but at the beginning. The resulting temperature profiles in this case are shown in Figure 2.15b. 

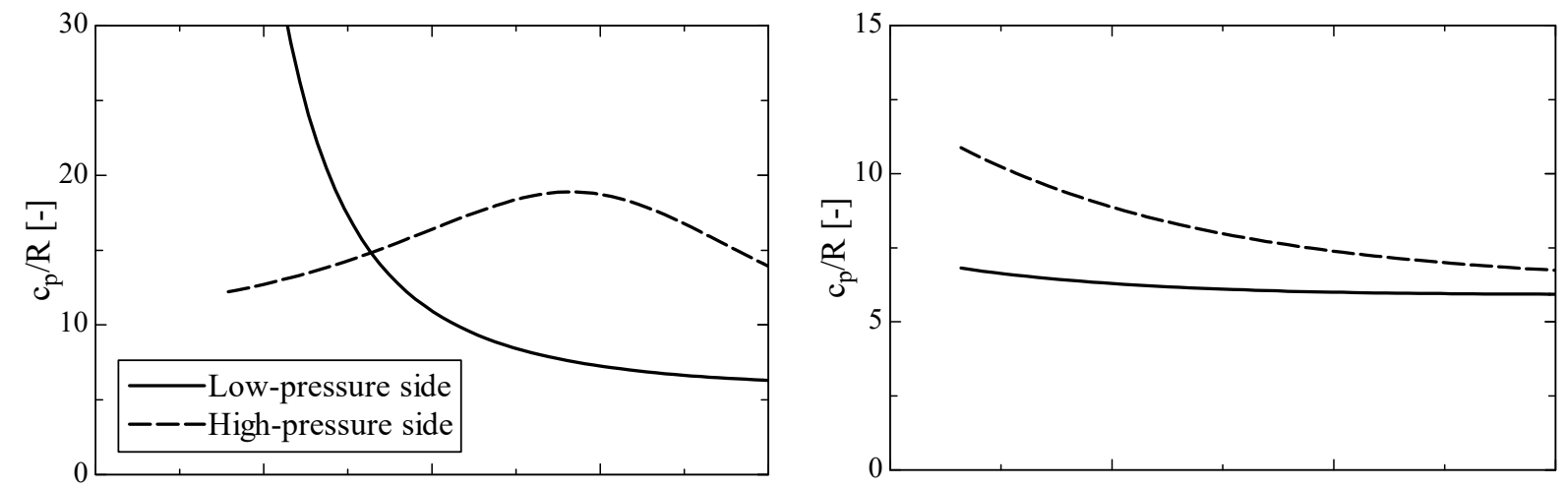

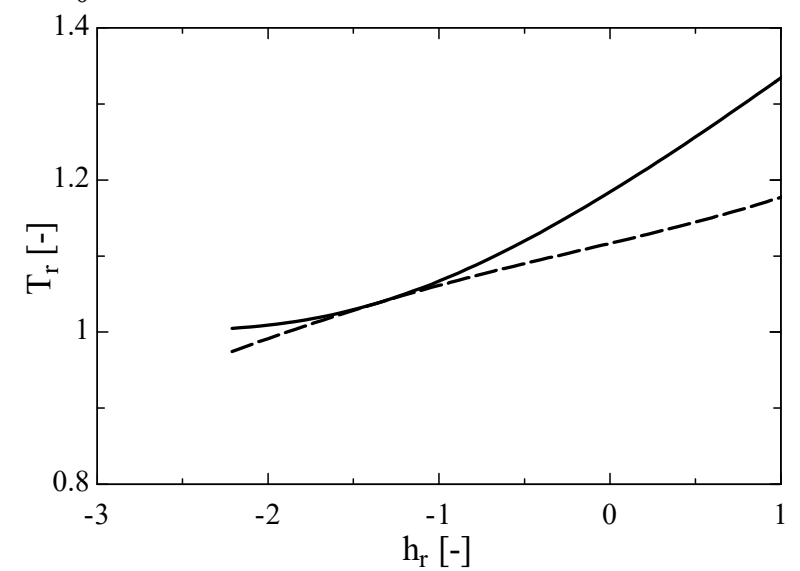

(a) $\min (d h / d P)_{T}$ inside the cycle

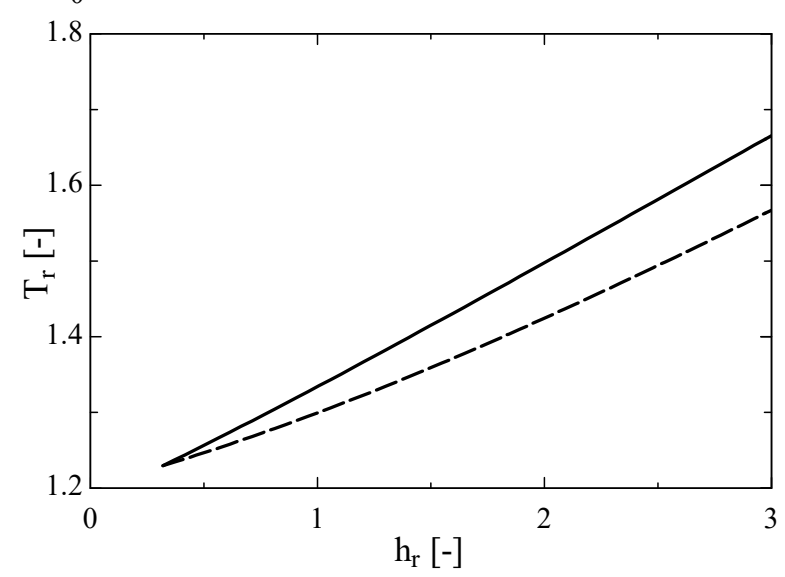

(b) $\min (d h / d P)_{T}$ outside the cycle

Figure 2.15. Dimensionless specific heat, $c_{p} / R$, and temperatures, $T_{r}$, in the high- and lowpressure sides of regeneration of a cycle with the minimum $(d h / d P)_{T}(\mathrm{a})$ inside the cycle and (b) outside the cycle

The next step in the analysis of the regeneration is to integrate the enthalpy balance along the whole phase. The balance between the high- and low-side pressures (HP and $L P)$ and the temperatures $T_{m}$ and $T_{t}$ is expressed in Equation 2.67.

$$
\int_{T_{m}}^{T_{t}} c_{P, H P} d T=\int_{T_{m}}^{T_{t}} c_{P, L P} d T+\int_{T_{m}}^{T_{t}} \Delta c_{P} d T=\int_{T_{m}}^{T_{t}} c_{P, L P} d T+\Delta h_{a}
$$

The low-pressure side would need an additional enthalpy increment, $\Delta h_{a}$, to heat the highpressure side up to the temperature $T_{t}$ due to the specific heat difference $\Delta c_{p}$ between the highand low-pressure sides. This means that there is a regeneration heat loss in the high-side pressure equal to $\Delta h_{a}$. This additional enthalpy increment, $\Delta h_{a}$, together with the heat needed to increase the temperature from $T_{t}$ to the turbine inlet temperature $T_{t, i n}$ is the total heat supplied by the primary heat, $q_{p h}$ :

$$
q_{p h}=\int_{T_{t}}^{T_{t, i n}} c_{P, H P} d T+\Delta h_{a}
$$


If the energy balance from Equation 2.67 is applied from $T_{m}$ to $T_{c}$ the result is that the lowpressure side needs an additional enthalpy increment, $\Delta h_{a, p c}$, to cool the low-pressure side down to $T_{c}$. Figure 2.16 shows a schematic diagram of the mentioned additional heats and its implication in the total heat supplied and extracted in the cycle. The total heat supplied by the primary heat is $q_{p h}$ and the heat extracted by the precooler $q_{p c}$. The regeneration limits are the blue lines parallel to the linearized isothermal line $T_{m}$ : one with the compressor outlet on the top and the other with the turbine outlet at the bottom. These lines are parallel due to the enthalpy balance in the regeneration phase.

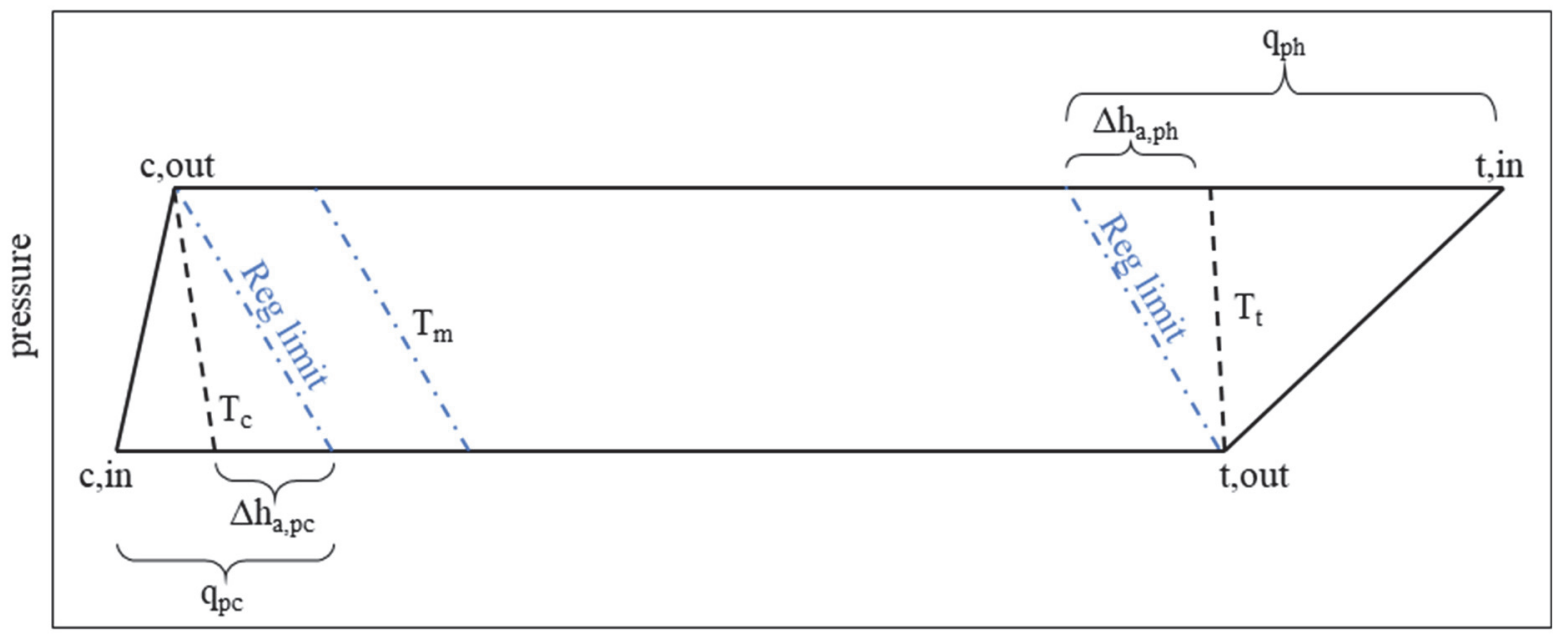

enthalpy

Figure 2.16. Schematic diagram of a pericritical regenerative cycle with the minimum $(d h / d P)_{T}$ inside, showing the additional enthalpy increments needed in primary heat and precooler, $\Delta h_{a, p h}$ and $\Delta h_{a, p c}$, due to the specific heat mismatch of the regeneration phase.

Figure 2.16 represents the case of a cycle with the minimum $(d h / d P)_{T}$ inside the cycle. If the cycle had the minimum $(d h / d P)_{T}$ outside the cycle, lines $T_{c}$ and $T_{m}$ would be the same, and would mark the left limit of the regeneration. Figure 2.17 shows a schematic diagram of this case. The right limit is parallel to the left limit with the turbine outlet as one of its points. The lines limiting the regeneration (blue lines) are parallel due to the enthalpy balance in the regeneration phase. Note that there is no additional enthalpy increment, $\Delta h_{a, p c}$, in the precooler because the $T_{c}$ and $T_{m}$ are the same line.

If the cycle worked in the ideal gas region, the isothermal lines would be vertical. Thus, there would not be regeneration heat loss $\Delta h_{a}$ and the heat needed by the ideal cycle, $q_{i g}$, would be equal to the turbine specific work, $w_{t}$ :

$$
q_{i g}=h_{t, \text { in }}-h_{t, o u t}=w_{t}
$$




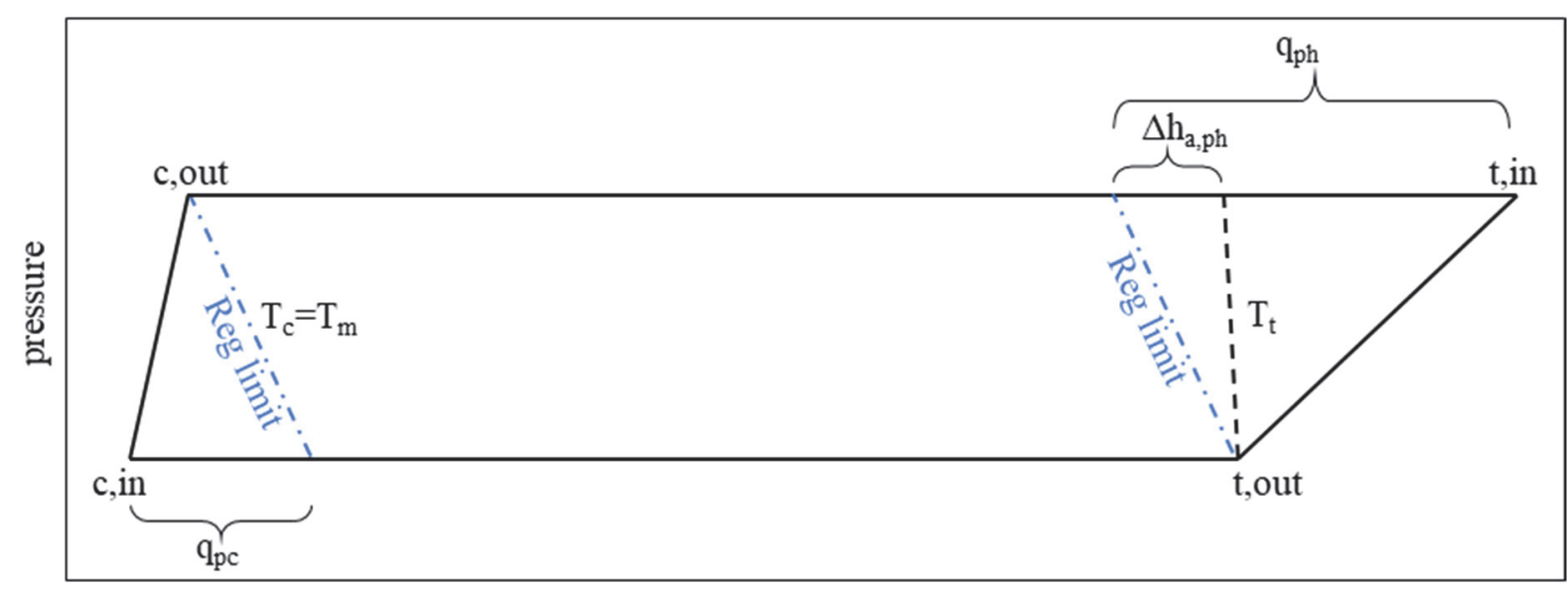

enthalpy

Figure 2.17. Schematic diagram of a pericritical regenerative cycle with the minimum $(d h / d P)_{T}$ outside the cycle, showing the additional enthalpy increment needed in primary heat, $\Delta h_{a, p h}$, due to the specific heat mismatch of the regeneration phase.

The difference between the heat needed in a cycle working at real gas conditions and the heat needed in an ideal cycle will be called $\Delta h_{r g}$. Thus, the heat needed by a pericritical cycle can be expressed as:

$$
q_{p h}=w_{t}+\Delta h_{r g}
$$

where $\Delta h_{r g}$ is the sum of $\Delta h_{a}$ plus the enthalpy variation of the isothermal $T_{t}, \Delta h_{T_{t}}$ :

$$
\Delta h_{r g}=\Delta h_{a}+\Delta h_{T_{t}}
$$

The enthalpy variation of the isothermal $T_{t}$ tends to zero when the turbine works close the ideal gas region at high temperatures. Thus, in these cases, Equation 2.70 can be approximated to:

$$
q=w_{t}+\Delta h_{a}
$$

This work will study cycles with the turbine working close to the ideal gas region. Thus, $\Delta h_{r g}$ and $\Delta h_{a}$ will be very similar and Equation 2.72 will be valid. These two terms will be referred to as regeneration heat loss or enthalpy variation due to real gas conditions.

Note that the enthalpy variation due to real gas conditions $\Delta h_{r g}$ is equal to the enthalpy variation of the isothermal $T m$. Thus, the value of $\Delta h_{r g}$ is the maximum enthalpy variation of an isothermal line during the regeneration, and it depends on the logarithmic factor of isobaric expansion $f_{P}$ in the same way than the derivative $(d h / d P)_{T}$. Figure 2.18 shows the value of $\Delta h_{r g}$ as a function of the compressor inlet temperature $T_{c, i n, r}$ for a pericritical cycle with the low-side pressure $P_{L, r}=1$ and different pressure ratios, $r$. The compressor inlet temperature 
$T_{c, i n, r}=1$ is the temperature corresponding to the pressure $P_{L, r}=1$ in the discontinuity line. Temperatures under the discontinuity line $\left(T_{c, i n, r}<1\right.$ in this case) involve small values of the coefficient $g$ and therefore low temperature increments during the compression. The result is a compressor outlet temperature lower than the temperature with the minimum $(d h / d P)_{T}$, such as in Figure 2.11, and therefore a cycle with the maximum possible value of $\Delta h_{r g}$ for a cycle with the same limiting pressures. For cycles with temperatures greater than the discontinuity line temperatures, the compressor outlet temperature is above the temperature with the minimum $(d h / d P)_{T}$. Thus, the minimum value of $(d h / d P)_{T}$ in the regeneration is the one corresponding to the compressor outlet temperature (such as in Figure 2.13), which involves lower values of $\Delta h_{\mathrm{rg}}$. In this case, higher compressor inlet temperatures involve greater temperatures $T_{m}$, which means a more vertical isothermal line and so lower $\Delta h_{r g}$ values.

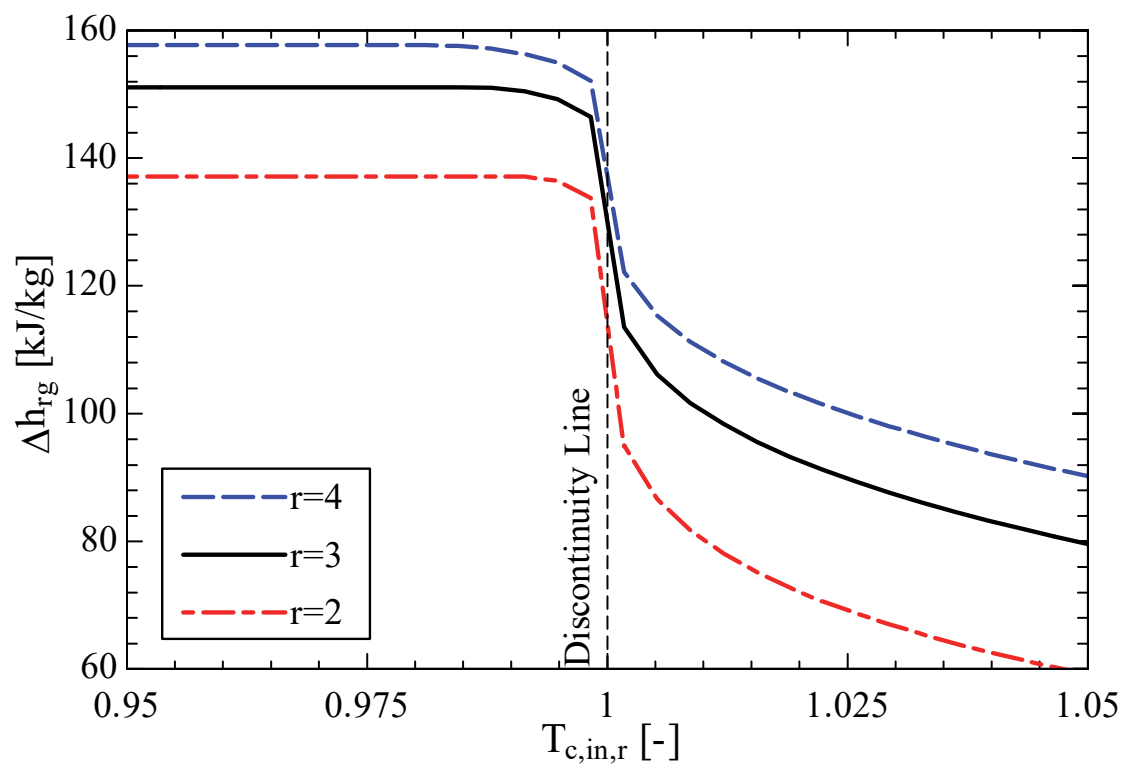

Figure 2.18. Maximum enthalpy variation of an isothermal line during regeneration, $\Delta h_{r g}$, as a function of the compressor inlet temperature, $T_{c, i n, r}$, for different pressure ratios

The behaviour of $\Delta h_{r g}$ is easier to understand with an example. Consider the case with pressure ratio $r=3$. Figure 2.12 showed that the temperature with the minimum $(d h / d P)_{T}$ in this case is $T_{\min , d h d P}=1.055$. When the compressor inlet temperature is $T_{c, i n, r}=0.95$, the outlet temperature is $T_{c, \text { out }, r}=0.99$, which is lower than $T_{\min , d h d P}=1.055$; and when the inlet temperature is $T_{c, i n, r}=1.05$, the outlet temperature is $T_{c, o u t, r}=1.34$, which is higher than $T_{\min , d h d P}=1.055$. The big difference in the outlet temperature involve a big difference in the value of $\Delta h_{r g}$.

Figure 2.18 shows that the value of $\Delta h_{r g}$ depends not only on the compressor inlet temperature, but also on the liming pressures. This dependency is represented in Figure 2.19 
for two compressor inlet temperatures, $T_{c, i n, r}=0.95$ and $T_{c, i n, r}=1.05$. The value of $\Delta h_{r g}$ increases with the pressure ratio. Both cases show the strongest variations at low pressure ratios and a threshold at large pressure ratios. The larger values of $\Delta h_{r g}$ in the case of $T_{c, i n, r}=0.95$ are due to the larger values of logarithmic factor of isobaric expansion $f_{P}$ along the isobars. These larger variations of $\Delta h_{r g}$ are especially relevant in the cycle performance at low pressure ratios, where the specific turbine work is small. The value of the turbine work $w_{t}$ can be compared with the enthalpy variation $\Delta h_{r g}$ in Figure 2.19. At low pressure ratios, the low specific turbine work and the large heat due to the high value of $\Delta h_{r g}$ will involve low efficiencies. The large turbine work at big pressure ratios will compensate the additional heat needed due to real gas conditions $\Delta h_{r g}$.

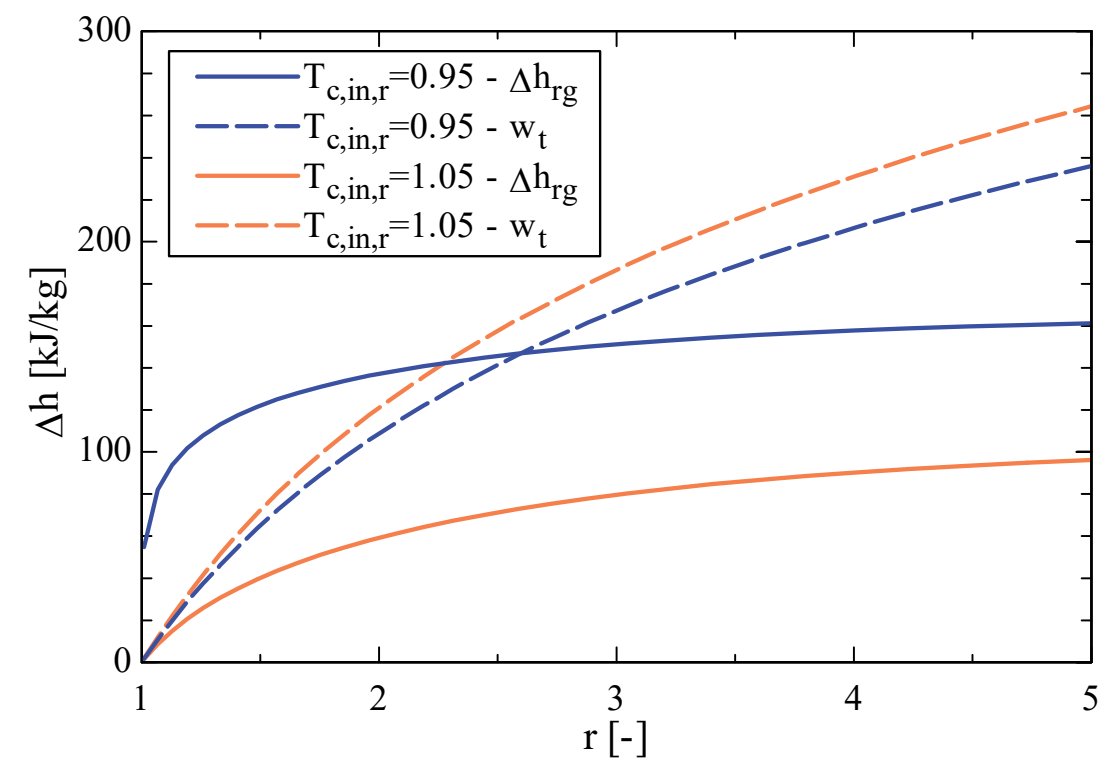

Figure 2.19. Maximum enthalpy variation of an isothermal line during regeneration, $\Delta h_{r g}$, and specific turbine work, $w_{t}$, as a function of the pressure ratio, $r$, for cycles with low-side pressure $P_{L, r}=1$ and different compressor inlet temperatures $T_{c, i n, r}$

The relation between the parameters $\Delta h_{r g}$ and $w t$ will be useful to characterize the cycle performance. The parameter $\Delta h_{r g} / w_{t}$ expresses the additional heat needed by the cycle due to real gas features with respect to the turbine work. But it can also be seen as the additional heat needed by the cycle with respect to the heat that a cycle with ideal gas would need. Thus, the term $\Delta h_{r g} / w_{t}$ will be referred to as the dimensionless additional heat needed by the cycle due to real gas features. Figure 2.20 shows $\Delta h_{r g} / w_{t}$ as a function of the pressure ratio, $r$, in a pericritical cycle with the same conditions than in Figure 2.19. The difference in the $\Delta h_{r g} / w_{t}$ values is mainly due the $\Delta h_{r g}$ value, which depends on the position of the compressor with respect to the discontinuity line. 


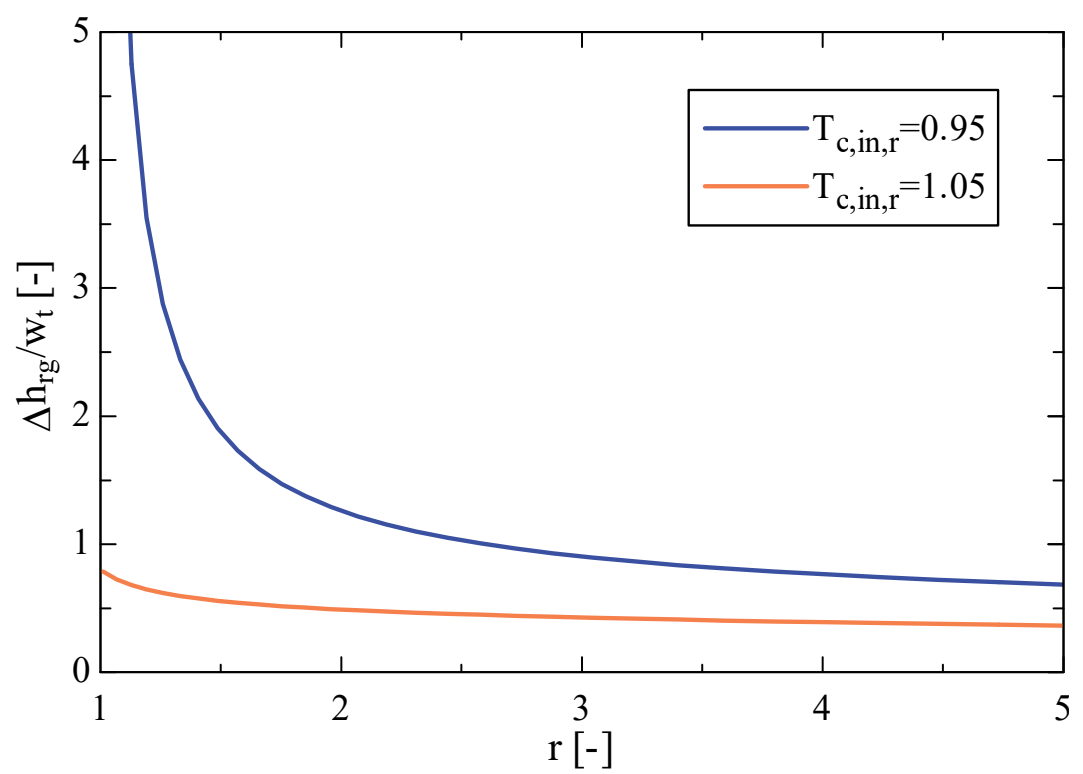

Figure 2.20. Dimensionless additional enthalpy, $\Delta h_{r g} / w_{t}$, as a function of the pressure ratio, $r$, in a pericritical cycle with low-side pressure $P_{L, r}=1$ and compressor inlet temperatures

$$
T_{c, i n, r}=0.95 \text { and } T_{c, i n, r}=1.05
$$

The main advantage of positioning the compressor at low temperatures was the low compression work in relation to the turbine work (see Figure 2.9). However, Figure 2.20 shows that this advantage is counteracted by the large additional heats needed at low pressure ratios. The next section analyses the influence of these two features in the cycle thermal efficiency. The results from this analysis will guide the optimization of pericritical cycles.

\subsubsection{Thermal efficiency}

The thermal efficiency is defined as the net specific work divided by the heat supply:

$$
\eta_{t h}=\frac{w_{t}-w_{c}}{q}
$$

The heat supply can be expressed as the sum of the turbine work $w_{t}$ and the enthalpy variation due to real gas features $\Delta h_{r g}$. Thus, Equation 2.73 can be transformed into Equation 2.74 .

$$
\eta_{t h}=\frac{w_{t}-w_{c}}{w_{t}+\Delta h_{r g}}=\frac{1-\frac{w_{c}}{w_{t}}}{1+\frac{\Delta h_{r g}}{w_{t}}}
$$

Feher (1968) used a similar equation to define the thermal efficiency. The thermal efficiency depends now on two dimensionless variables: $w_{c} / w_{t}$ and $\Delta h_{r g} / w_{t}$. These two variables have been analysed in previous section. The former will be referred to as dimensionless 
compression work and the latter as dimensionless additional enthalpy supplied due to real gas conditions. These parameters depend on the thermodynamic properties of the fluid, and therefore on the position of the thermodynamic region where they are located.

Note that if the cycle worked in the ideal gas region, the enthalpy variation due to real gas features would be $\Delta h_{r g \text {,ideal }}=0$. Then the expression for the thermal efficiency would be:

$$
\eta_{t h, i d e a l}=1-\frac{w_{c, i d e a l}}{w_{t, i d e a l}}
$$

And if the value of $w_{c} / w_{t}$ at ideal conditions expressed in Equation 2.58 is introduced in Equation 2.75 then:

$$
\eta_{t h}=1-\frac{T_{c, i n}}{T_{t, i n}} \cdot r^{\frac{\gamma-1}{\gamma}}
$$

The thermal efficiency at ideal conditions would only depend on the Carnot factor $T_{t, i n} / T_{c, i n}$, the pressure ratio $r$, and the adiabatic coefficient $\gamma$.

Figure 2.21 shows the thermal efficiency, $\eta_{t h}$, together with the parameters $w_{c} / w_{t}$ and $\Delta h_{r g} / w_{t}$ from Figure 2.9 and Figure 2.20 for the cases with compressor inlet temperatures $T_{c, i n, r}=0.95$ and $T_{c, i n, r}=1.05$. The abscissa represents the pressure ratio $r$ that, in this case, is equal to the high-side pressure $P_{H}$, as the low-side pressure is $P_{L, r}=1$. The parameters $w_{c} / w_{t}$ and $\Delta h_{r g} / w_{t}$ play a different role in the thermal efficiency. When the pressure ratio increases, $\Delta h_{r g} / w_{t}$ makes the efficiency grow and $w_{c} / w_{t}$ makes it decrease. In the case of $T_{c, i n, r}=1.05$ at low pressure ratios, the weight of $\Delta h_{r g} / w_{t}$ is greater than the weight of $w_{c} / w_{t}$, and therefore the thermal efficiency increases. However, when the pressure ratio gets to $r=3$ then the trend changes and the efficiency starts to decrease. The same happens in the case of $T_{c, i n, r}=0.95$, but the change of trend in the efficiency cannot be seen in the figure because it happens at a pressure ratio around $r=8$.

When the compressor inlet temperature is $T_{c, i n, r}=0.95$, the lower values of compression work $w_{c} / w_{t}$ in comparison to those of $T_{c, i n, r}=1.05$ come at the expense of larger values of additional enthalpy $\Delta h_{r g} / w_{t}$. The benefits obtained in the compression due to the lower values of the compressibility factor, $z$, are counteracted by the high values of logarithmic factor of isobaric expansion, $f_{P}$, during the phase of heat regeneration. The result is that, in order to take real advantage of the low compression work $w_{c} / w_{t}$, the cycle with compressor inlet temperature $T_{c, i n, r}=0.95$ needs large pressure ratios. 

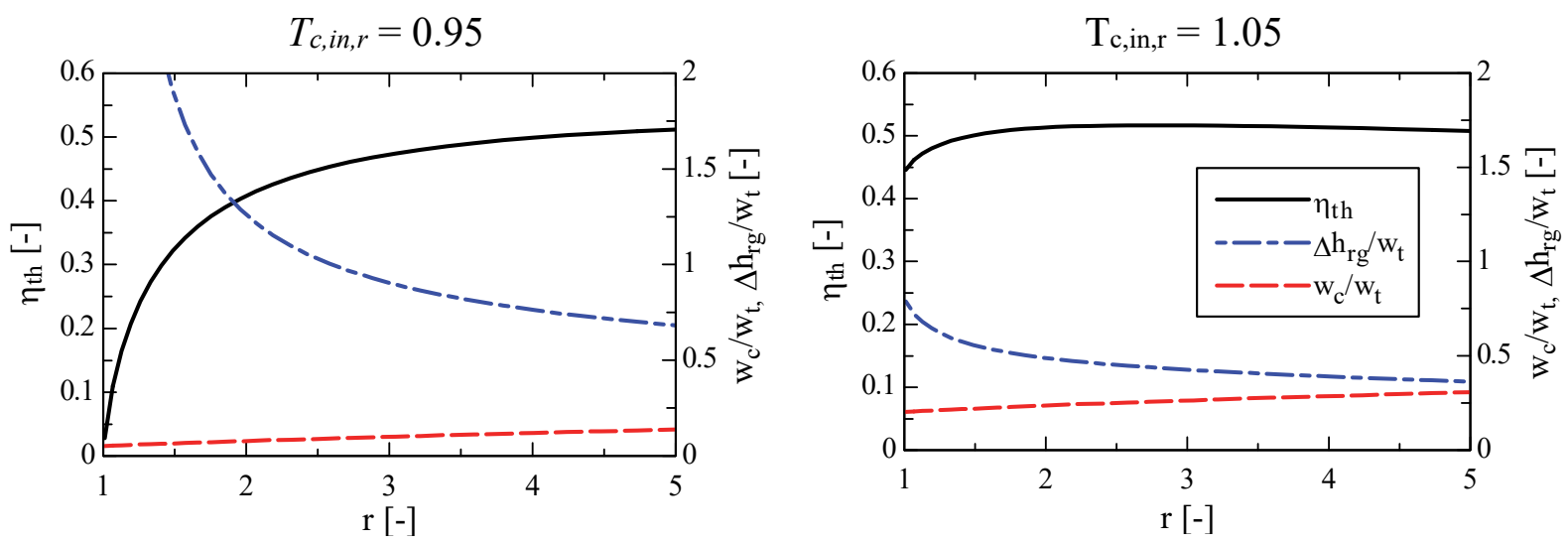

Figure 2.21. Dimensionless compression work, $w_{c} / w_{t}$, dimensionless additional enthalpy, $\Delta h_{r g} / w_{t}$, and thermal efficiency, $\eta_{t h}$, as a function of the pressure ratio, $r$, in a pericritical cycle with low-side pressure $P_{L, r}=1$

Figure 2.21 shows that raising the high-side pressure do not necessarily involve higher efficiencies when the low-side pressure is constant. But even if this were the case, the highside pressure is usually limited by tecno-economic reasons. Thus, the main objective will be to find the highest efficiency within a design window defined by an upper limit in pressure.

The compressor inlet temperature and pressure define the compressor position with respect to the discontinuity line. In the cases previously studied, the compressor defined by $P_{L, r}=1$ and $T_{c, i n, r}=0.95$ worked above the discontinuity line (liquid region), and the compressor defined by $P_{L, r}=1$ and $T_{c, i n, r}=1.05$ below (gaseous region). If the cycle were defined by the high-side pressure instead of by the low-side pressure, the low-side pressure would be a variable that would set the position of the cycle with respect to the discontinuity line.

Figure 2.22 shows six different pericritical cycles in a P-h diagram working with a high pressure $P_{H, r}=3$. Three of these cycles have the compressor working to the left of the discontinuity line (the discontinuity line crosses the low-pressure side), and three of them have the compressor to the right. The position with respect to the discontinuity line depends on the low-side pressure and on the compressor inlet temperature.

The effect of the compressor crossing the discontinuity line (DL) by lowering the low-side pressure is shown in Figure 2.23. Thermal efficiency, $\eta_{t h}$, together with the parameters $w_{c} / w_{t}$ and $\Delta h_{r g} / w_{t}$, are depicted for cycles with compressor inlet temperatures $T_{c, i n, r}=0.95$ and $T_{c, i n, r}=1.05$, and a fixed high-side pressure $P_{H, r}=3$. The abscissa represents the pressure ratio instead of the low-side pressure. Increasing the pressure ratio means now that the lowpressure side goes down along an isothermal line in the same way than in Figure 2.22. 


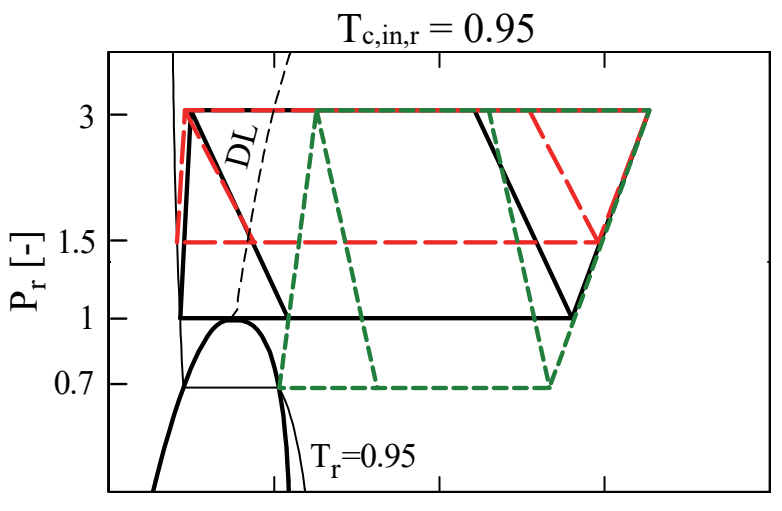

$\mathrm{h}_{\mathrm{r}}[-]$

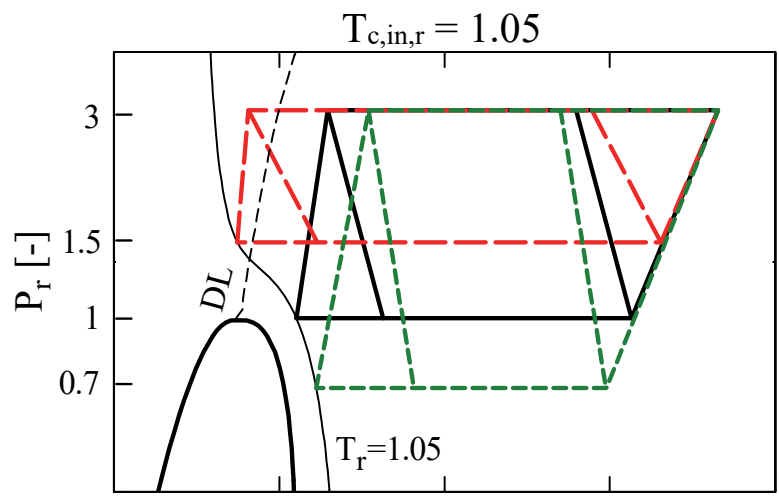

$\mathrm{h}_{\mathrm{r}}[-]$

Figure 2.22. P-h diagram of pericritical cycles working at high-side pressure $P_{H, r}=3$ and different low-side pressures and compressor inlet temperatures
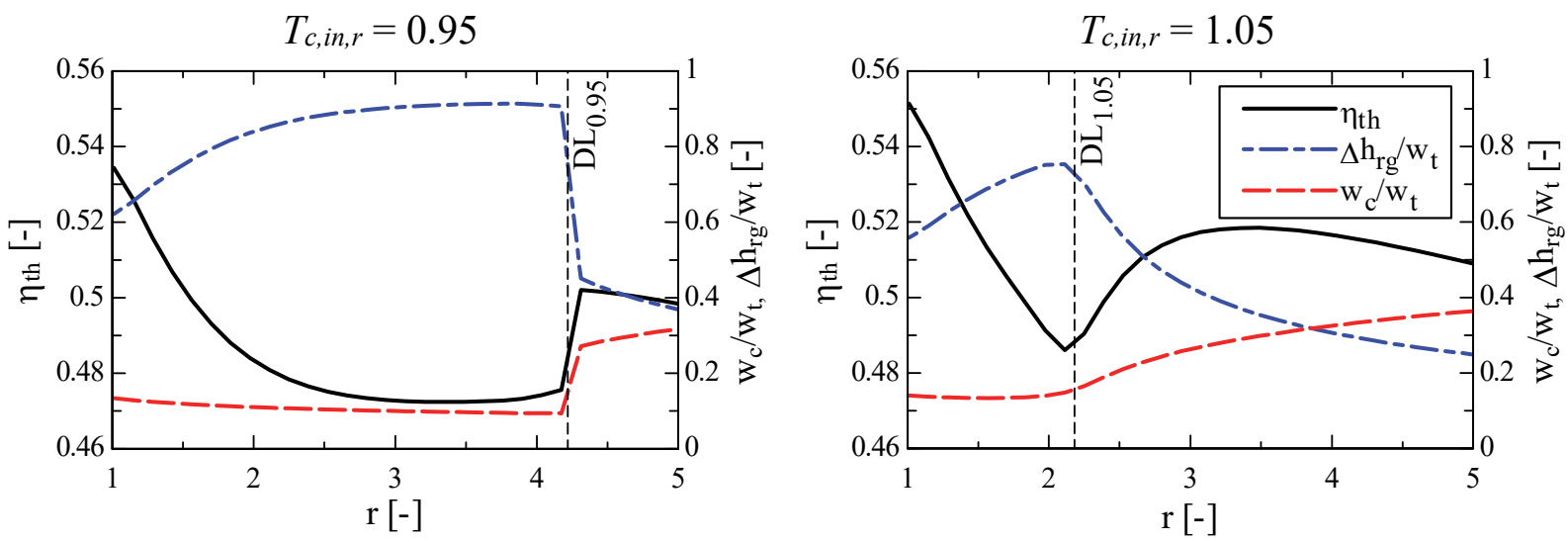

Figure 2.23. Dimensionless compression work, $w_{c} / w_{t}$, dimensionless additional enthalpy, $\Delta h_{r g} / w_{t}$, and thermal efficiency, $\eta_{t h}$, as a function of the pressure ratio, $r$, in a pericritical cycle with high-pressure side $P_{H, r}=3$

The vertical dashed line in Figure 2.23 represents the pressure ratio at which the compressor inlet would touch the discontinuity line (DL). This pressure ratio is $r=4.2$ when the compressor inlet temperature is $T_{c, i n, r}=0.95$, and $r=2.2$ when the compressor inlet temperature is $T_{c, i n, r}=1.05 . D L_{0.95}$ and $D L_{1.05}$ are the points where the discontinuity line crosses the isothermal lines $T_{c, i n, r}=0.95$ and $T_{c, i n, r}=1.05$, respectively.

Both compressor inlet temperatures present a similar behavior: when $r$ grows, the parameter $w_{c} / w_{t}$ decreases up to a point, where it starts to increase. Just the opposite happens to the parameter $\Delta h_{r g} / w_{t}$. The maximum value of $\Delta h_{r g} / w_{t}$ is achieved when $f_{P}$ values are maximum, which happens when the compressor inlet coincides with the discontinuity line (DL). In both cases the discontinuity line separates the two regions already explained: the left side, with low $w_{c} / w_{t}$ values and high $\Delta h_{r g} / w_{t}$ values, and the right side, with high $w_{c} / w_{t}$ values and low 
$\Delta h_{r g} / w_{t}$ values. The main difference is the location of the discontinuity line $(r=4.2$ and $r=$ 2.2) and the stronger variation of the results around the discontinuity line in the case of $T_{c, i n, r}=0.95$. While the compression work $w_{c} / w_{t}$ starts to increase at pressure ratios above $r=2.2$ when the compressor inlet temperature is $T_{c, i n, r}=1.05, w_{c} / w_{t}$ continues going down when the temperature is $T_{c, i n, r}=0.95$. The result is a smaller compression work in the discontinuity line of the cycle with compressor inlet temperature $T_{c, i n, r}=0.95$ mainly due to the longer range of pressures between the high-side pressure and the discontinuity line at this temperature. In the same way, the $\Delta h_{r g} / w_{t}$ value is greater in the discontinuity line of the cycle with compressor inlet temperature $T_{c, i n, r}=0.95$.

Note that the results obtained for pressure ratios under $r=2.2$ and above $r=4.2$ are similar regardless of the compressor inlet temperature. The compressor works in the liquid region in the former case and in the gaseous region in the latter case regardless of the compressor inlet temperature. In between $r=2.2$ and $r=4.2$, compressors with inlet temperature $T_{c, i n, r}=0.95$ work in the liquid-like region, and compressors with inlet temperature $T_{c, i n, r}=1.05$ in the gaslike region. Thus, the main differences between the results at these compressor inlet temperatures are in this region.

Decreasing the pressure ratio in the left part of Figure 2.23 reduces the values of $\Delta h \mathrm{rg} / \mathrm{wt}$, which involves a benefit in the thermal efficiency. The maximum efficiency is obtained when values of $\Delta h_{r g} / w_{t}$ cannot be decreased further at pressure ratio $r=1$. This is the result of a cycle following isentropic lines during compression and expansion and isobars during heating and cooling. However, this is only an ideal case. In a real case, the machinery will come along with inefficiencies that will affect the thermal efficiency and its optimal configuration. The following section studies the effect of machinery inefficiencies depending on the limiting conditions such as pressure and temperature.

\subsection{Analysis of real pericritical cycles}

\subsubsection{The real pericritical cycle}

Pericritical cycles have four phases: compression, heating, expansion and cooling. The simplest system would have a machine for each phase. However, pericritical cycles must include also a regenerator in order to minimize the heat supplied by the heat source and the heat extracted by heat sink. Figure 2.24 shows a diagram of a simple regenerative pericritical 
cycle. The word "simple" is used to highlight that the system can undergo modifications to increase the efficiency as it will be seen in the next chapter.

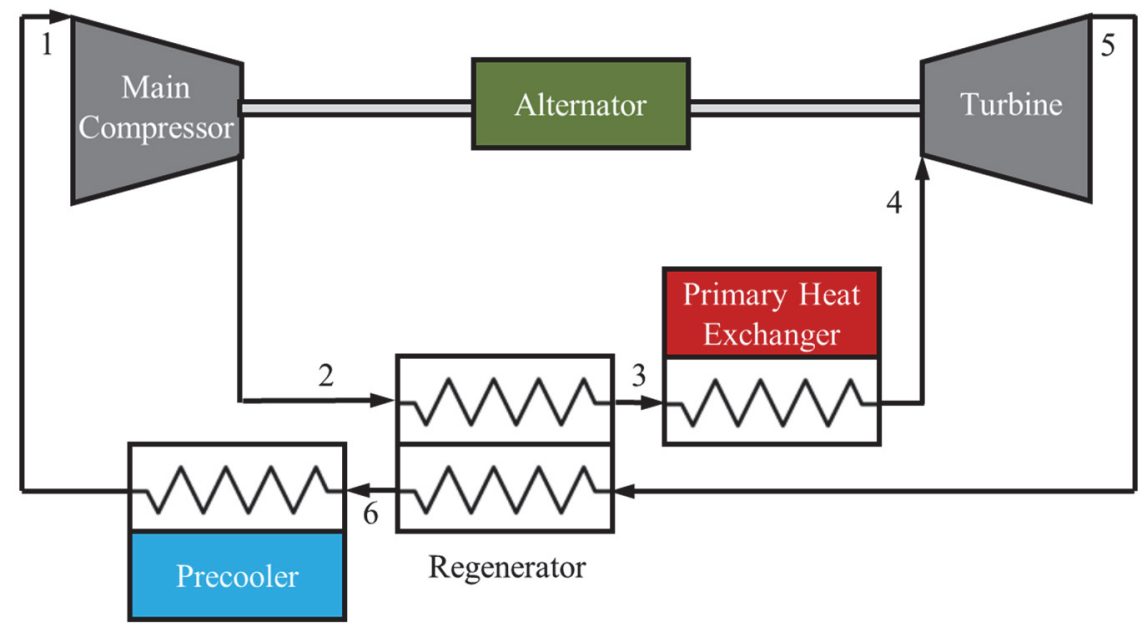

Figure 2.24. Diagram of a simple regenerative pericritical cycle (inspired by Dyreby (2014))

Equations 2.77-2.81 represent the different phases of a simple regenerative pericritical cycle by means of enthalpy increments. These phases are: compression work $\left(w_{c}\right)$, regenerator heat exchange $\left(q_{r e g}\right)$, primary heat $\left(q_{p h}\right)$, turbine work $\left(w_{t}\right)$, heat released by the precooler $\left(q_{p c}\right)$.

$$
\begin{gathered}
w_{c}=h_{2}-h_{1} \\
q_{r e g}=h_{3}-h_{2}=h_{5}-h_{6} \\
q_{p h}=h_{4}-h_{3} \\
w_{t}=h_{4}-h_{5} \\
q_{p c}=h_{6}-h_{1}
\end{gathered}
$$

Figure 2.25 shows an example of simple regenerative pericritical cycle in h-P and T-s diagram. Continuous lines represent the cycle limits, and dashed lines the regeneration limits. The purpose of showing a cycle with low-side pressure $P_{L, r}=1$ is to remind that pericritical cycles may have the low-side pressure above and below the critical pressure.

The cycle is limited by the high- and low-side pressures, $P_{H}$ and $P_{L}$. However, these pressures are not constant along the cycle due to pressure losses. Pressure losses imply that the turbine pressure ratio is lower than the compressor pressure ratio. Thus, $P_{H}$ and $P_{L}$ are the maximum and the minimum pressure of the cycle, which correspond to the compressor outlet and compressor inlet pressures. 

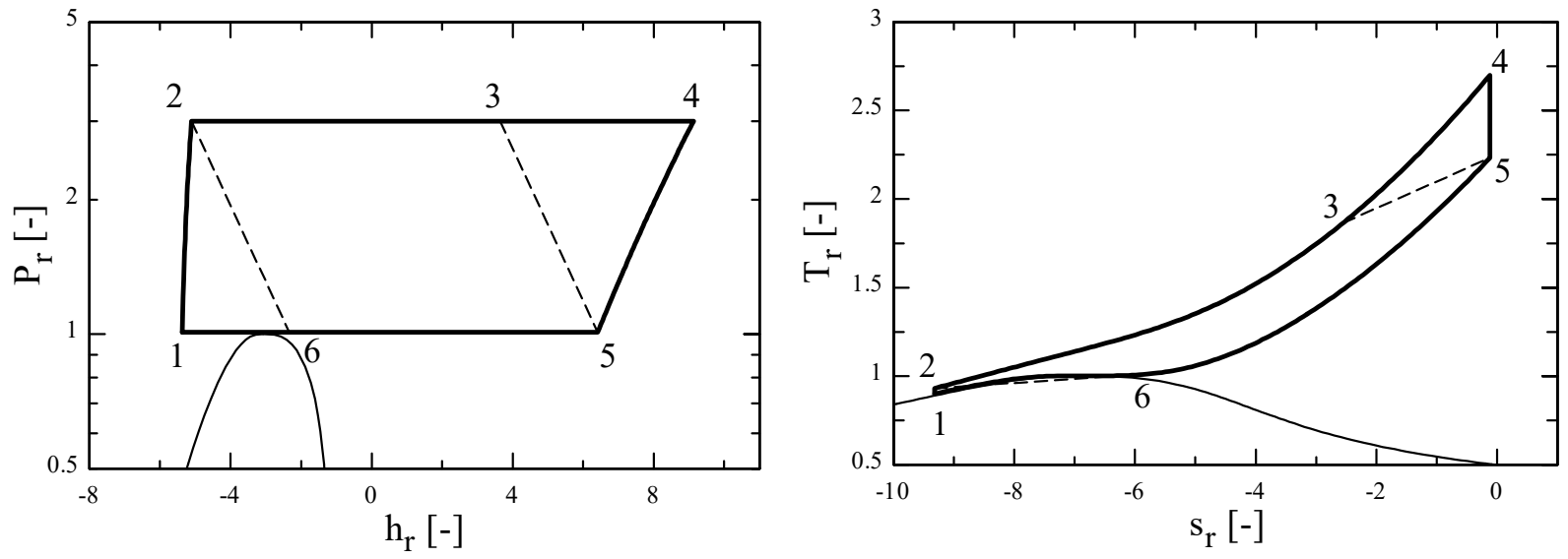

Figure 2.25. Example of simple regenerative pericritical cycle in diagrams P-h (left) and T-s (right). The numbers mark the beginning and end of each phase.

The compressor and turbine inlet pressures and temperatures define points 1 and 4 . If the compressor and turbine were ideal turbomachines, the outlet points $2, \mathrm{~s}$ and $5, \mathrm{~s}$ would be the intersection of the corresponding pressures and isentropic lines. But the isentropic efficiencies of compressor and turbine, $\eta_{c}$ and $\eta_{t}$, defined in Equations 2.82 and 2.83 , deteriorate its behaviour resulting in points 2 and 5 .

$$
\begin{gathered}
\eta_{c}=\frac{h_{2, s}-h_{1}}{h_{2}-h_{1}} \\
\eta_{t}=\frac{h_{4}-h_{5}}{h_{4}-h_{5, s}}
\end{gathered}
$$

The low-pressure part of the cycle between points 6-5 heats the high-pressure part between points 2-3 through an intermediate heat exchanger (IHX). If the IHX were infinitely large, the temperature difference between the high- and the low-pressure sides in the pinch point would be zero, and the heat exchanged would be the maximum possible, $\Delta h_{r e g, m a x}$. However, a real IHX cannot exchange infinite heat. The effectiveness, $\varepsilon$, will define how much heat, $\Delta h_{\text {reg, a }}$ real IHX exchanges in comparison to the maximum possible, $\Delta h_{\text {reg,max: }}$

$$
\varepsilon=\frac{\Delta h_{r e g}}{\Delta h_{r e g, \max }}
$$

If the heat exchanged in the regeneration decreases, the limiting lines 2-6 and 3-5 will be more inclined in the P-h diagram. Remember that the enthalpy variation of these lines was defined as $\Delta h_{r g}$. Thus, the value of $\Delta h_{r g}$ in a real cycle will be greater than in an ideal cycle, which involves a greater heat supply. 
In summary, compression, expansion and heat regeneration come along with inefficiencies. These inefficiencies can be divided into three groups depending on the effect that they have in in the thermal efficiency:

- Turbomachinery: Isentropic efficiencies increase ideal compression work and decrease ideal turbine work in the following way: $w_{c, \text { ideal }} / \eta_{c}$ and $w_{t, \text { ideal }} \cdot \eta_{t}$, where $\eta_{c}<1$ and $\eta_{t}<1$.

- Heat transfer in the intermediate heat exchanger: Heat transfer in an intermediate heat exchanger involves a larger enthalpy not regenerated in the following way: $\Delta h_{\text {rg, ideal } /} \eta_{H X}$ where $\eta_{H X}<1$.

- Pressure loss in primary heat exchanger, intermediate heat exchanger and precooler: Turbine work decreases due to the lower pressure difference between inlet and outlet in the following way: $\eta_{P} \cdot w_{t}$ where $\eta_{P}<1$.

Equation 2.85 shows the effect of these inefficiencies in the definition of thermal efficiency.

$$
\eta_{t h}=\frac{1-\frac{w_{c}}{w_{t}}}{1+\frac{\Delta h_{r g}}{w_{t}}}=\frac{1-\frac{w_{c, \text { ideal }}}{w_{t, \text { ideal }}} \frac{1}{\eta_{c} \eta_{t} \eta_{P}}}{1+\frac{\Delta h_{r g, \text { ideal }}}{w_{t, \text { ideal }}} \frac{1}{\eta_{t} \eta_{P} \eta_{H X}}}
$$

Note that the inefficiency corresponding to the pressure loss, $\eta_{P}$, can be considered together with the turbine efficiency, $\eta_{t}$, as they have the same effect in the thermal efficiency.

The same turbomachine efficiency will be used to compare liquid and gas regions. Although the compression will behave differently in these two regions, this analysis allows to compare the cycle performance depending on the turbomachine potential. In a similar way, although the IHX size will be different in two cycles with the same effectiveness but different limiting pressures, the goal of this analysis is to measure the cycle potential.

\subsubsection{Analysis of inefficiencies}

The effectiveness of the intermediate heat exchanger, $\varepsilon$, will define the inefficiency of the regeneration phase. The turbomachines inefficiencies will be agglutinated into one value to simplify the analysis. It will be considered that compressor efficiency, $\eta_{c}$, and turbine efficiency, $\eta_{t}$, are equal. Moreover, as the purpose of this first analysis is to study the effect of the inefficiencies, the pressure loss is not studied separately because it affects the thermal efficiency in the same way than the turbine efficiency (see Equation 2.85). Thus, a lower value of $\eta_{t}$ will indicate a combination of lower turbine work and higher pressure loss. 
Figure 2.26 shows the influence of the inefficiencies, $\eta_{c}$ and $\varepsilon$, in a pericritical cycle with high-side pressure $P_{H, r}=3$, Carnot factor $T_{t, i n} / T_{c, i n}=3$, and compressor inlet temperatures $T_{c, i n, r}=0.95$ (to the left) and $T_{c, i n, r}=1.05$ (to the right). The thermal efficiency, $\eta_{t h}$, and the parameters used to define it, $w_{c} / w_{t}$ and $\Delta h_{r g} / w_{t}$, are represented as a function of the pressure ratio, $r$. The results show similar trends regardless of the compressor inlet temperature: pericritical cycles with the compressor working in the liquid region have lower compression work $w_{c} / w_{t}$ and larger regeneration heat loss $\Delta h_{r g} / w_{t}$ than cycles with the compressor working in the gaseous region.
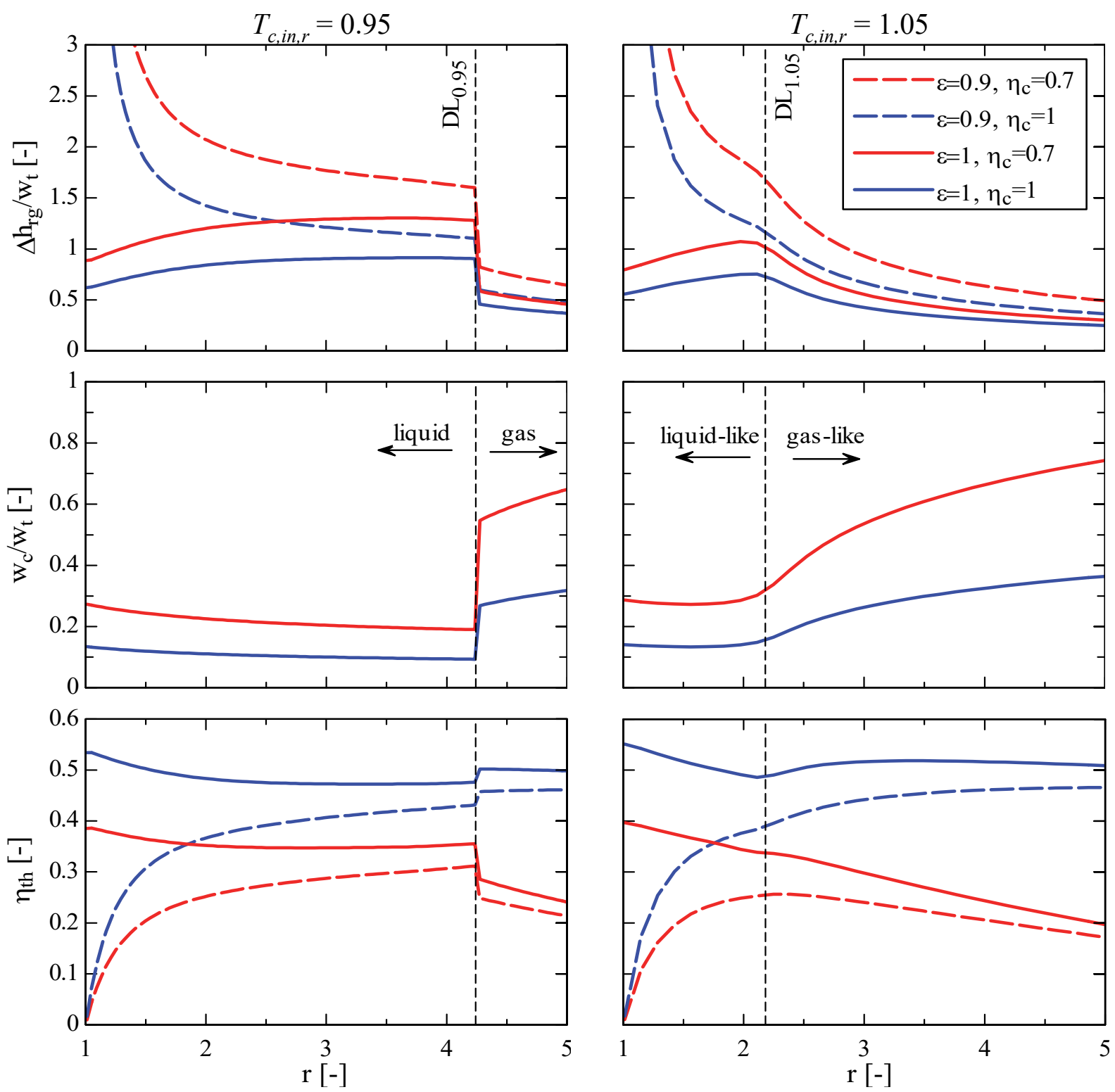

Figure 2.26. Parameters $\Delta h_{r g} / w_{t}$ and $w_{c} / w_{t}$, and thermal efficiency $\eta_{t h}$ as a function of the pressure ratio, $r$, in pericritical cycles with high-pressure side $P_{H, r}=3$ and different compressor inlet temperatures and inefficiencies 
There are four cases represented in each figure resulting from the combination of the inefficiencies $\eta_{c}=0.7, \eta_{c}=1, \varepsilon=0.9$ and $\varepsilon=1$. The turbomachine inefficiencies $\eta_{c}$ affect the parameter $w_{c} / w_{t}$ independently of the IHX effectiveness. Thus, there are only two study cases for the parameter $w_{c} / w_{t}$, one for $\eta_{c}=0.7$ (in red) and the other for $\eta_{c}=1$ (in blue). Reducing the turbomachine efficiency increases the value of $w_{c} / w_{t}$. The smaller the value of $w_{c} / w_{t}$, the smaller its increase is (in absolute terms). Therefore, cycles with low turbomachine efficiency will take more advantage of the regions with lower $w_{c} / w_{t}$ values in order to increase it as less as possible. The lowest $w_{c} / w_{t}$ values are found in the liquid region. Cycles with the compressor working in the liquid region will have greater advantages in cycles with low turbomachine efficiencies.

The turbine efficiency also affects the result of $\Delta h_{r g} / w_{t}$. Lowering the turbine efficiency decreases the turbine specific work, and therefore increases the parameter $\Delta h_{r g} / w t$. Lowering the effectiveness also increases the term $\Delta h_{r g} / w t$, and it has a greater effect than the turbine efficiency, especially in cycles with low pressure ratios in which the heat regeneration is more relevant. The increase of $\Delta h_{r g} / w_{t}$ is smaller (in absolute terms) when the pressure ratios are large. Cycles with larger pressure ratios will have greater advantages in cycles with low effectiveness.

In general terms, the IHX inefficiency decreases the thermal efficiency at low pressure ratios and the turbomachine inefficiency do it at high pressure ratios. The optimum pressure ratios are greater in cycles with the IHX inefficiencies dominating over the turbomachine inefficiencies (such as the case with $\varepsilon=0.9$ and $\eta_{c}=1$ ), and on the contrary, the optimum pressure ratios are smaller when the turbomachine inefficiencies dominate over the IHX inefficiencies (such as the case with $\varepsilon=1$ and $\eta_{c}=0.7$ ). In cycles where all the inefficiencies have a similar effect, such as the case with $\varepsilon=0.9$ and $\eta_{c}=0.7$, the optimum pressure ratio are around the discontinuity line.

The results obtained with compressor inlet temperatures $T_{c, i n, r}=0.95$ and $T_{c, i n, r}=1.05$ are very similar for pressure ratios under $r=2.2$ and above $r=4.2$. The main differences are in between these two pressure ratios. In the liquid region close to the discontinuity line, the parameter $w_{c} / w_{t}$ is smaller when the compressor inlet temperature is $T_{c, i n, r}=0.95$ than when it is $T_{c, i n, r}=1.05$. Therefore, cycles compressor inlet temperature $T_{c, i n, r}=0.95$ will have greater advantages when the turbomachine efficiency is low. Moreover, the IHX inefficiency (the effectiveness) affects less the cycle thermal efficiency at larger pressure ratios. Thus, cycles with larger pressure ratio between the high-side pressure and the discontinuity line, such as in 
the case of compressor inlet temperature $T_{c, i n, r}=0.95$, have greater advantages when the effectiveness is low. The result can be seen in the case of low effectiveness $\varepsilon=0.9$ and low turbomachine efficiency $\eta_{c}=0.7$ of Figure 2.26, where the cycle with compressor inlet temperature $T_{c, i n, r}=0.95$ achieve a higher maximum thermal efficiency.

Especial attention must be paid to the ideal case $\left(\eta_{c}=1\right.$ and $\left.\varepsilon=1\right)$. The curve of thermal efficiency has two peaks: one peak corresponds to the maximum efficiency at pressure ratio $r=1$, and the other at a larger pressure ratio (which depends on the compressor inlet temperature). If the effectiveness were slightly lower than $\varepsilon=1$, the second peak could overcome the first one, and therefore, result in a very different optimum pressure ratio. Figure 2.27 shows pericritical cycles with high IHX effectiveness and different values of turbomachine efficiency. The optimum pressure ratio is close to one when the turbomachine efficiency is $\eta_{c}=0.8$. However, the optimum pressure ratio jumps to around $r=4(r=4.2$ at $T_{c, i n, r}=0.95$ and $r=3.8$ at $\left.T_{c, i n, r}=1.05\right)$ when the turbomachine efficiency is $\eta_{c}=1$. At high effectiveness values, different turbomachine efficiencies may imply strong variations in the optimum pressure ratio.
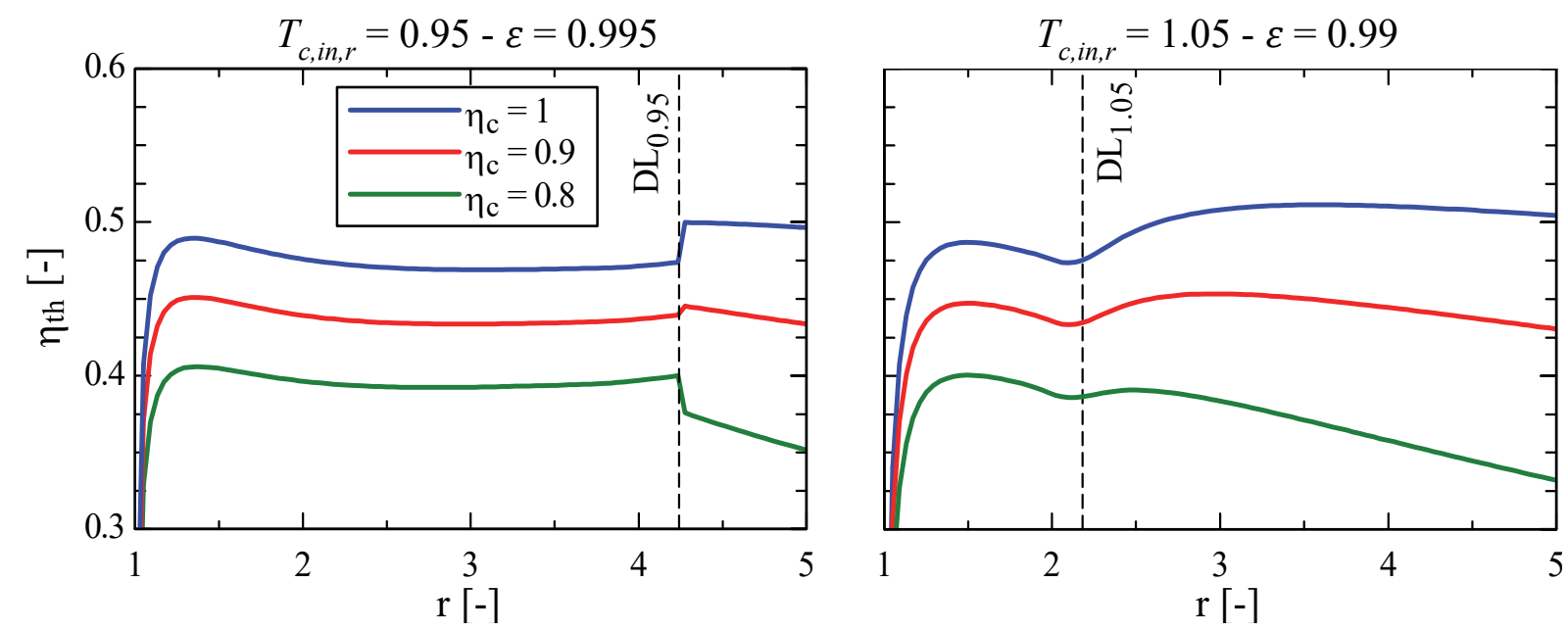

Figure 2.27. Thermal efficiency $\eta_{t h}$ as a function of the pressure ratio, $r$, in pericritical cycles with high IHX effectiveness

The optimum pressure ratio depends on the relation between inefficiencies and compressor inlet temperature. Figure 2.28 shows the optimum pressure ratio, $r_{o p t}$, as a function of the IHX effectiveness, $\varepsilon$, in pericritical cycles with compressor inlet temperatures $T_{c, i n, r}=0.95$ and $T_{c, i n, r}=1.05$. The results are shown for different values of turbomachine efficiencies, $\eta_{c}$. Cycles with low effectiveness and high turbomachine efficiency achieve its maximum efficiency at large pressure ratios. The optimum pressure ratio decreases when the IHX 
effectiveness raises and when the turbomachine efficiency lowers. This decrease stops at the discontinuity line and there is a wide range of inefficiency values in which the optimum pressure ratio remains constant $(r=4.2$ when the compressor inlet temperatures is $\left.T_{c, i n, r}=0.95\right)$ or almost constant $(r=2.2$ when the compressor inlet temperatures is $\left.T_{c, i n, r}=1.05\right)$. These optimum ratios correspond to cycles with the compressor inlet working in the discontinuity line. Although it is inappreciable in Figure 2.28, some of these cycles get to reduce slightly the pressure ratio and cross the discontinue line when the turbomachine efficiency decreases. For example, when the compressor inlet temperature is $T_{c, i n, r}=0.95$ and the effectiveness $\varepsilon=0.9$, cycles with turbomachine efficiencies $\eta_{c}=0.9$ and $\eta_{c}=1$ have the compressor inlet pressure just below the discontinuity line, and the cycles with lower turbomachine efficiencies, just above. When the effectiveness increases, the optimum pressure ratio crosses the discontinuity line only at high effectiveness and with an abrupt change due to the behavior shown in Figure 2.27.
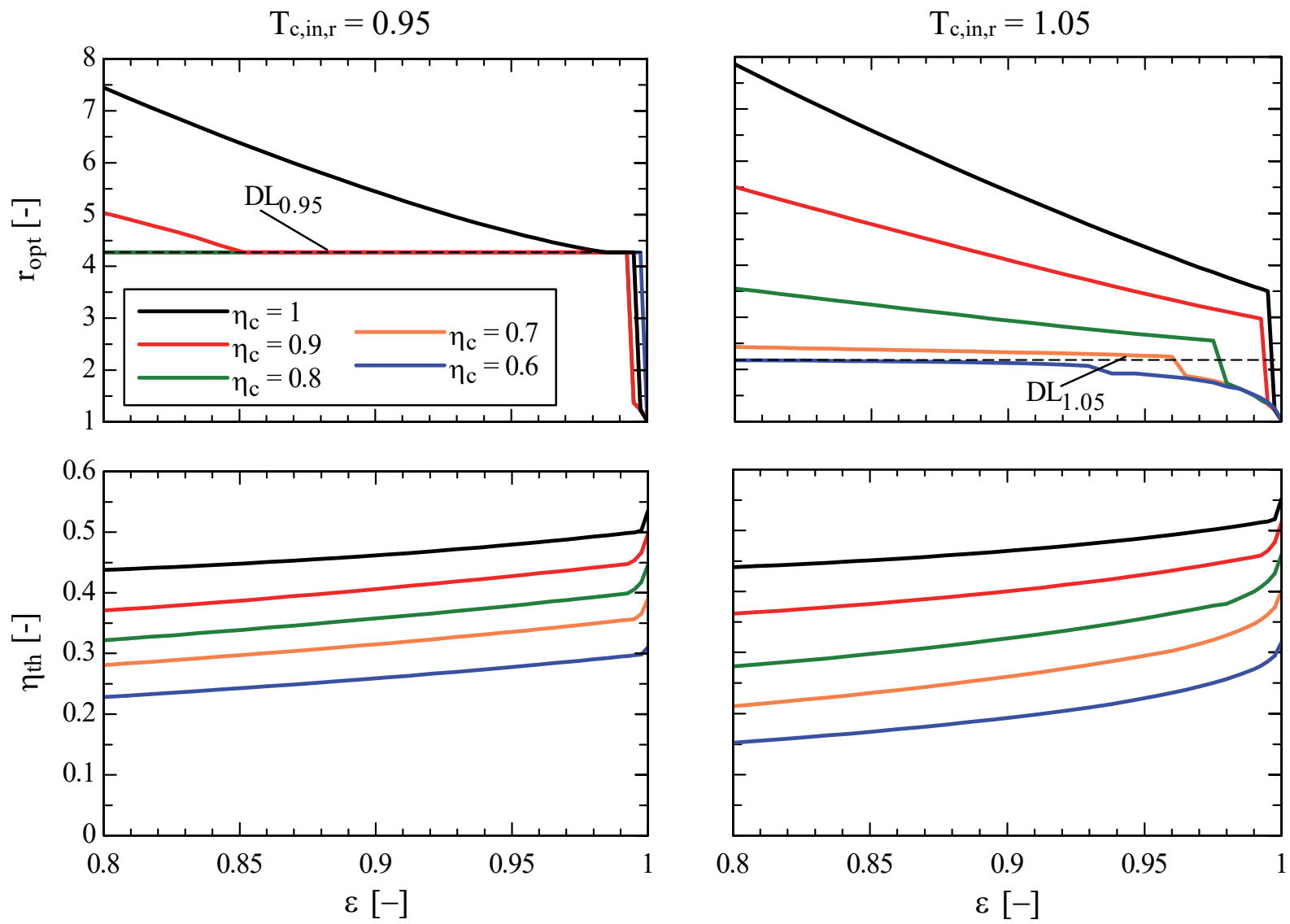

Figure 2.28. Optimum pressure ratio, $r_{o p t}$, and thermal efficiency, $\eta_{t h}$, as a function of the IHX effectiveness, $\varepsilon$, for different values of turbomachine efficiencies, $\eta_{c}$.

Pressure ratio lines corresponding to turbine efficiencies $\eta_{c}=0.9$ and $\eta_{c}=1$ show a similar behavior in cycles with compressor inlet temperatures $T_{c, i n, r}=0.95$ and $T_{c, i n, r}=1.05$ until the 
lines from the case with $T_{c, i n, r}=0.95$ touch the discontinuity line $D L_{0.95}$. From that effectiveness value onwards, the lines in the case of $T_{c, i n, r}=0.95$ stays together with the discontinuity line $D L_{0.95}$. In the case of $T_{c, i n, r}=1.05$, the lines continue going down towards the discontinuity line $D L_{1.05}$, although it is not reached. Lines corresponding to turbine efficiencies $\eta_{c}=0.6$ and $\eta_{c}=0.7$ stay in the discontinuity lines $D L 0.95$ and $D L_{1.05}$. They follow a specific optimum pressure ratio when the compressor inlet temperature is $T_{c, i n, r}=0.95$, and a range of pressure ratios when the temperature is $T_{c, i n, r}=1.05$. This is because the discontinuity line at temperatures lower than the critical temperature is a line, but it is a band (called discontinuity band) at greater temperatures.

The discontinuity line can be seen as a potential field attracting the low-side pressure of pericritical cycles. The strength of this attraction is proportional to the IHX effectiveness and inversely proportional to the turbomachine efficiency. The only exception is when the IHX is almost ideal (effectiveness close to one). In this case, the optimum pressure ratio tends to one as it was explained in Figure 2.27. However, these configurations would imply huge heat exchangers and therefore they are a priori discarded as reliable solution.

Thermal efficiencies obtained with the optimum pressure ratios are also shown in Figure 2.28. The thermal efficiency increases linearly with the turbomachine efficiency, $\eta_{c}$. It also increases linearly with the IHX effectiveness, $\varepsilon$, except when this value is close to one. In this case, it increases more rapidly for a small range of effectiveness values. Thermal efficiencies in the case of $T_{c, i n, r}=0.95$ are higher in cycles with large inefficiencies (low values of $\eta_{c}$ and $\varepsilon)$. These cycles compensate the inefficiencies with larger pressure ratios in the liquid region. The thermal efficiencies of the cycles with compressor inlet temperature $T_{c, i n, r}=0.95$ and $T_{c, i n, r}=1.05$ become very similar when the inefficiencies are small (high values of $\eta_{c}$ and $\varepsilon$ ). And in the limit of $\eta_{c}=1$ and $\varepsilon=1$, the cycle with compressor inlet temperature $T_{c, i n, r}=1.05$ is $3 \%$ more efficient.

One of the reasons of the difference in efficiency between the cycles with compressor inlet temperature $T_{c, i n, r}=0.95$ and $T_{c, i n, r}=1.05$ is the different range of pressures between the highside pressure and the discontinuity line, which involve differences in the parameter $w_{c} / w t$. The parameter $w_{c} / w_{t}$ is multiplied by $1 / \eta_{c} \eta_{t}$ in the equation of thermal efficiency. Thus, the cycles with high inefficiencies take advantage of the low $w_{c} / w_{t}$ values in the liquid region to counteract the low turbomachine efficiency. The greater range of pressures between the highside pressure and the discontinuity line at temperature $T_{c, i n, r}=0.95$ involve lower achievable values of $w_{c} / w_{t}$, and so higher thermal efficiencies. The distance between high-side pressure 
and discontinuity line depends on the compressor inlet temperature. Therefore, compressor inlet temperature and high-side pressure will be highly related in the cycle performance.

\subsubsection{Analysis of temperature and pressure}

Previous calculations have set the high-pressure side to $P_{H, r}=3$. Nevertheless, it is interesting to study the behavior of pericritical cycles with other pressure values. Figure 2.29 analyses the influence of the high-side pressure in pericritical cycles with inefficiencies $\eta_{c}=\eta_{t}=0.85$ and $\varepsilon=0.95$. The cycle thermal efficiency, $\eta_{t h}$, is represented as a function of the low-side pressure, $P_{L, r}$ for different high-side pressures $\left(P_{H, r}=1, P_{H, r}=3\right.$ and $\left.P_{H, r}=5\right)$ and compressor inlet temperatures $\left(T_{c, i n, r}=0.95\right.$ and $\left.T_{c, i n, r}=1.05\right)$. The pressures corresponding to the points of the discontinuity line with temperatures $T_{r}=0.95$ and $T_{r}=1.05$ are represented as $D L 0.95$ and $D L_{1.05}$.

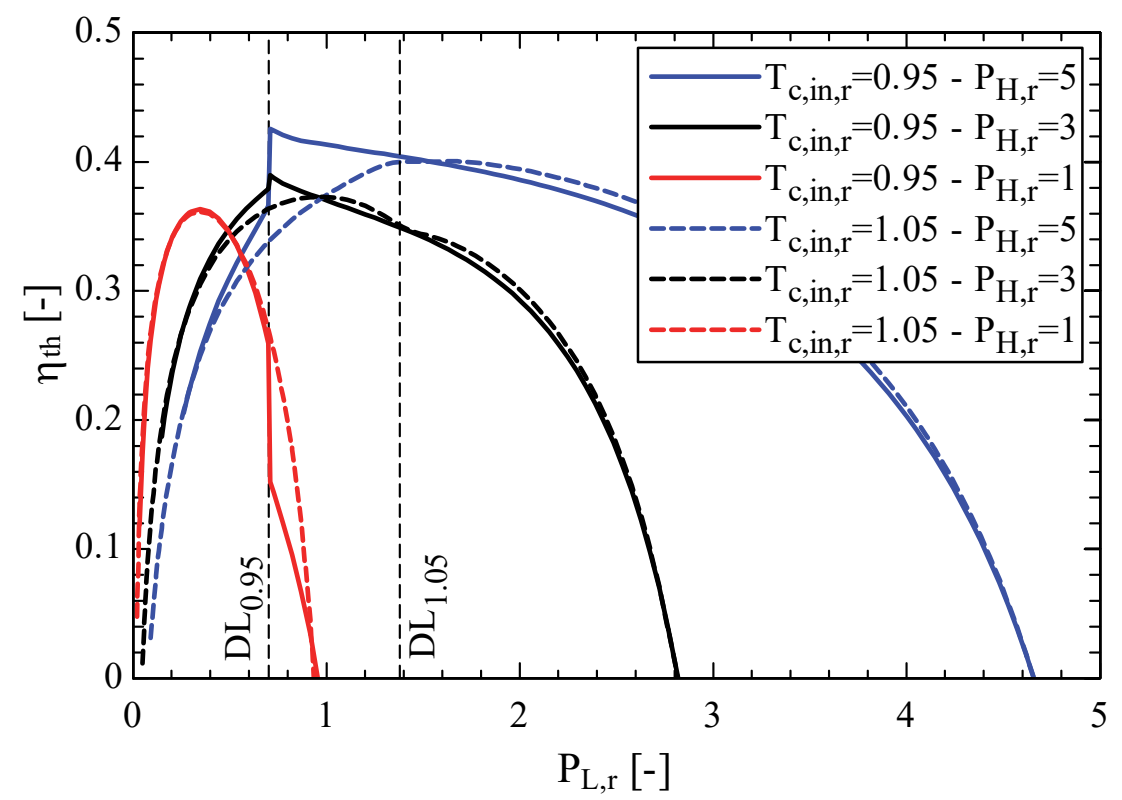

Figure 2.29. Thermal efficiency, $\eta_{t h}$, as a function of the low-side pressure, $P_{L, r}$, for different high-side pressures, $P_{H, r}$, and compressor inlet temperatures, $T_{c, i n, r}$

The results with compressor inlet temperatures $T_{c, i n, r}=0.95$ (continuous lines) and $T_{c, i n, r}=1.05$ (discontinuous lines) are very similar to the left of $D L 0.95$ and to the right of $D L_{1.05}$. The compressor works in the gas region in the former case and in the liquid region in the latter case, regardless of the compressor inlet temperature. In between $D L_{0.95}$ and $D L_{1.05}$, compressors with inlet temperature $T_{c, i n, r}=0.95$ work in the liquid region, and compressors with inlet temperature $T_{c, i n, r}=1.05$ in the gaseous region. The main differences occur in this region. 
When the compressor works in the liquid region (i.e. at pressures above the discontinuity line), lowering the low-side pressure reduces the compression work, $w_{c} / w_{t}$, which increases the thermal efficiency. If the low-side pressure goes down below the discontinuity line, the compression work $w_{c} / w_{t}$ increases, which has a negative effect in the thermal efficiency. The result is that the maximum thermal efficiency is obtained at low-side pressures just above the discontinuity line when the high-side pressures are $P_{H, r}=3$ and $P_{H, r}=5$ in the case of compressor inlet temperature $T_{c, i n, r}=0.95$ and when the pressure is $P_{H, r}=5$ in the case of $T_{c, i n, r}=1.05$. When the high-side pressures are lower, the behavior is different. The greater proximity between high-side pressure and discontinuity line involve high $\Delta h_{r g} / w_{t}$ values if the low-side pressure is above the discontinuity line. Lowering the low-side pressure below the discontinuity line reduces $\Delta h_{r g} / w_{t}$ in a way that has more influence in the thermal efficiency than the increasing compression work. The result is that the maximum efficiency occurs when the compressor works in the gas region.

When the high-side pressure is very close to the discontinuity line, such as in the case of $P_{H, r}=1$, the optimum low-side pressure is low, which means that the compressor position is close to ideal gas conditions. In this case, the efficiency only depends on the Carnot factor $T_{t, i n} / T_{c, i n}$, the pressure ratio $r$, and the adiabatic coefficient $\gamma$ (as shown in Equation 2.78). As all these parameters are the same in the cases of compressor inlet temperature $T_{c, i n, r}=0.95$ and $T_{c, i n, r}=1.05$ (remember that the Carnot factor is set to $T_{t, i n} / T_{c, i n}=3$ in all the calculations), the maximum efficiency is the same in both cases.

Compressor inlet temperature and high-side pressure are highly related in the cycle performance. Figure 2.30 shows thermal efficiency, $\eta_{t h}$, and optimum low-side pressure, $P_{L, o p t, r}$, as a function of the high-pressure side, $P_{H, r}$, for different compressor inlet temperatures, $T_{c, i n, r}$. For low high-side pressures, the cycles work at low low-side pressures in the gaseous region. When the high-side pressure increases, the low-side pressure does it too until a limit. This limit corresponds to the compressor working in the discontinuity line. From this point on, the optimum low-side pressures $P_{L, o p t, r}$ is constant. The high-side pressure at which this limit is reached depends on the compressor inlet temperature, $T_{c, i n, r}$. The lower the compressor inlet temperature, the lower the high-side pressure from which the compressor works in the discontinuity line. This occurs because a minimum distance between the highside pressure and the discontinuity line is required to have the low-side pressure working on the discontinuity line. This distance is reached before by the lower compressor inlet temperatures. 


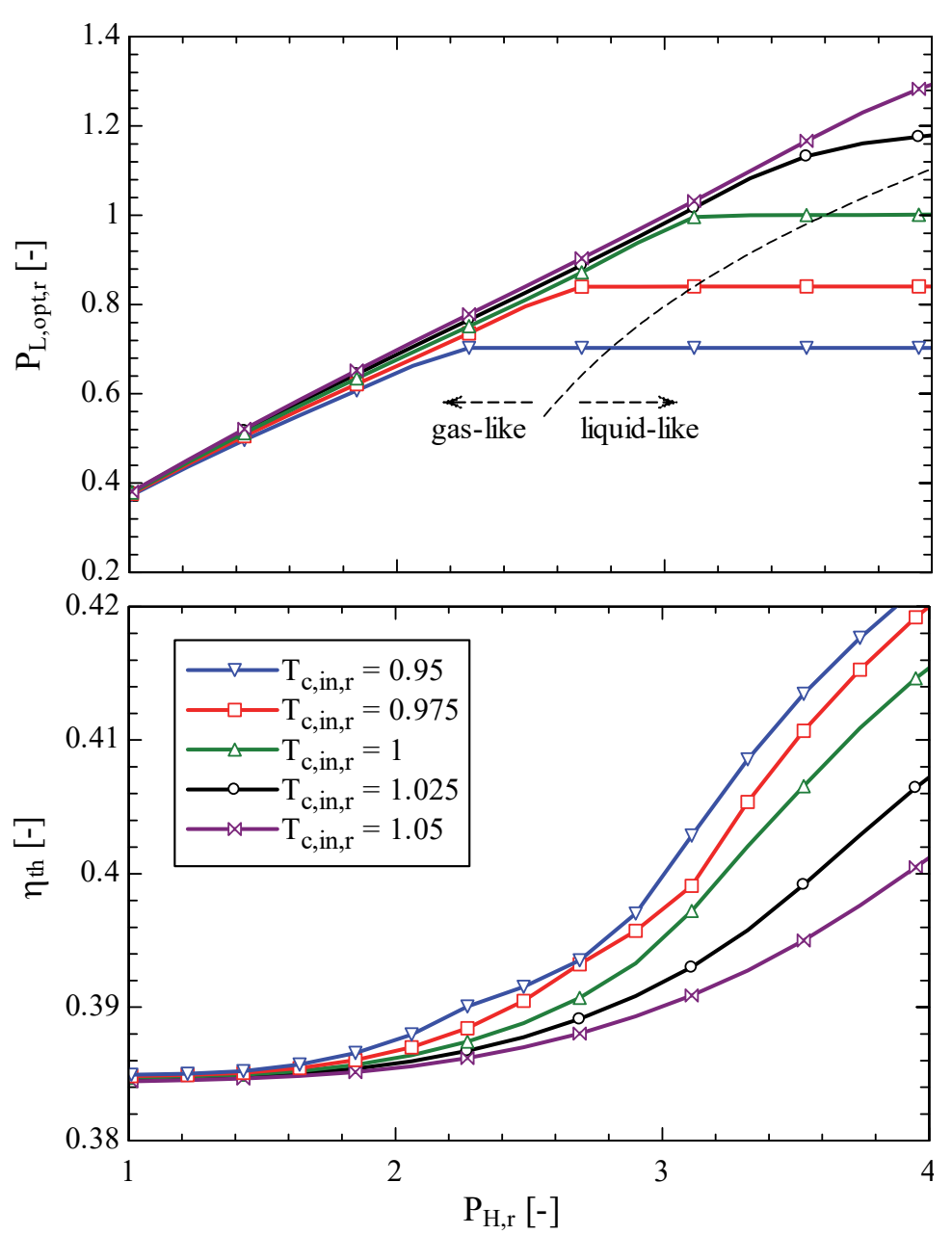

Figure 2.30. Thermal efficiency, $\eta_{t h}$, and optimum low-side pressure, $P_{L, o p t, r}$, as a function of high-pressure side, $P_{H, r}$, for different compressor inlet temperatures, $T_{c, i n, r}$

The low-side pressures $P_{L, o p t, r}$ corresponding to the discontinuity line for each temperature are the horizontal lines. These lines are perfectly horizontal when the compressor inlet temperature is below the critical temperature, and slightly inclined when it is above. The dashed line crossing these horizontal lines separate liquid and gas regions. This means that, to the left of this line, the cycle take advantage of the low $\Delta h_{\mathrm{rg}} / w_{t}$ values despite the high $w_{c} / w_{t}$ values, and to the right, just the opposite. This differentiation will be very important in next chapter, where the enthalpy variation due to real gas features, $\Delta h_{r g}$, will be processed to increase the cycle performance.

The thermal efficiency is very similar at all compressor inlet temperatures when the high-side pressure is low (close to the critical pressure). The reason is that these cycles work in the ideal gas region and the parameters on which the efficiency depends (Carnot factor $T_{t, i n} / T_{c, i n}$, pressure ratio $r$, and adiabatic coefficient $\gamma$ ) are the same at all the compressor inlet temperatures. Increasing the high-side pressure involves higher optimum low-side pressures, 
which gets away the compressor from the ideal gas region and approaches it to the discontinuity line. The result is that the thermal efficiency increases by raising the high-side pressure. At low compressor inlet temperatures the efficiency starts to increase earlier with the high-side pressure raise. For example, for a compressor inlet temperature $T_{c, i n, r}=0.95$, the efficiency begins to increase at around a high-side pressure $P_{H, r}=2$, while for an inlet temperature $T_{c, i n, r}=1.05$, it begins to increase at around $P_{H, r}=3$. This means that, in order to benefit from the high-side pressure raise, the pressure ratio between the high-side pressure and the discontinuity line must be greater than $r=2.8$ for the temperature $T_{c, i n, r}=0.95$ and greater than $r=2.2$ for the temperature $T_{c, i n, r}=1.05$. Once the efficiency starts to increase, it continues increasing till high-side pressures greater than those shown in Figure 2.30. Pressures above $P_{H, r}=4$ are not considered because they will frequently involve very expensive systems.

Note that Figure 2.30 represents the behavior of the most common cycles nowadays: gas turbines and steam Rankine cycles. If the high-side pressure is low, then the cycle works in the ideal gas region, which corresponds to the behavior of gas turbines. On the other hand, if the high-side pressure is high enough to locate the compressor in the liquid region and the temperature is below the critical temperature, then the cycle would be like a steam Rankine cycle.

Figure 2.30 shows that cycles with lower compressor inlet temperature achieve higher thermal efficiencies when the high-side pressure increases. These results have been calculated with the system inefficiencies $\eta_{c}=\eta_{t}$, $=0.85$ and $\varepsilon=0.95$, which are common values in the analysis of regenerative cycles. Figure 2.28 showed that, for more inefficient IHXs and turbomachines, the benefit of lower compressor inlet temperatures would be even greater, and smaller for more efficient IHXs and turbomachines. If IHXs and turbomachines were both almost ideal, the tendency could change, and higher compressor inlet temperatures could achieve slightly higher thermal efficiencies.

\subsection{Working fluid}

The results shown in this chapter have been obtained with the thermodynamic properties of $\mathrm{CO}_{2}$. Nonetheless, pericritical cycles could work with other fluids. In order to work in a pericritical cycle, the fluid must be able to be cooled down to temperatures between $T_{r}=0.9$ and $T_{r}=1.1$. If the minimum achievable temperatures by cooling at ambient conditions are 
between $300 \mathrm{~K}$ and $320 \mathrm{~K}$ then the critical temperature of the candidate fluids must be between $275 \mathrm{~K}$ and $350 \mathrm{~K}$ to match the mentioned reduced temperatures. Thus, fluids must be classified according to its critical temperature in order to identify candidates for pericritical cycles. Figure 2.31 shows some relevant fluids classified as a function of its critical temperature, $T_{c r}$, and also of its critical pressure, $P_{c r}$.

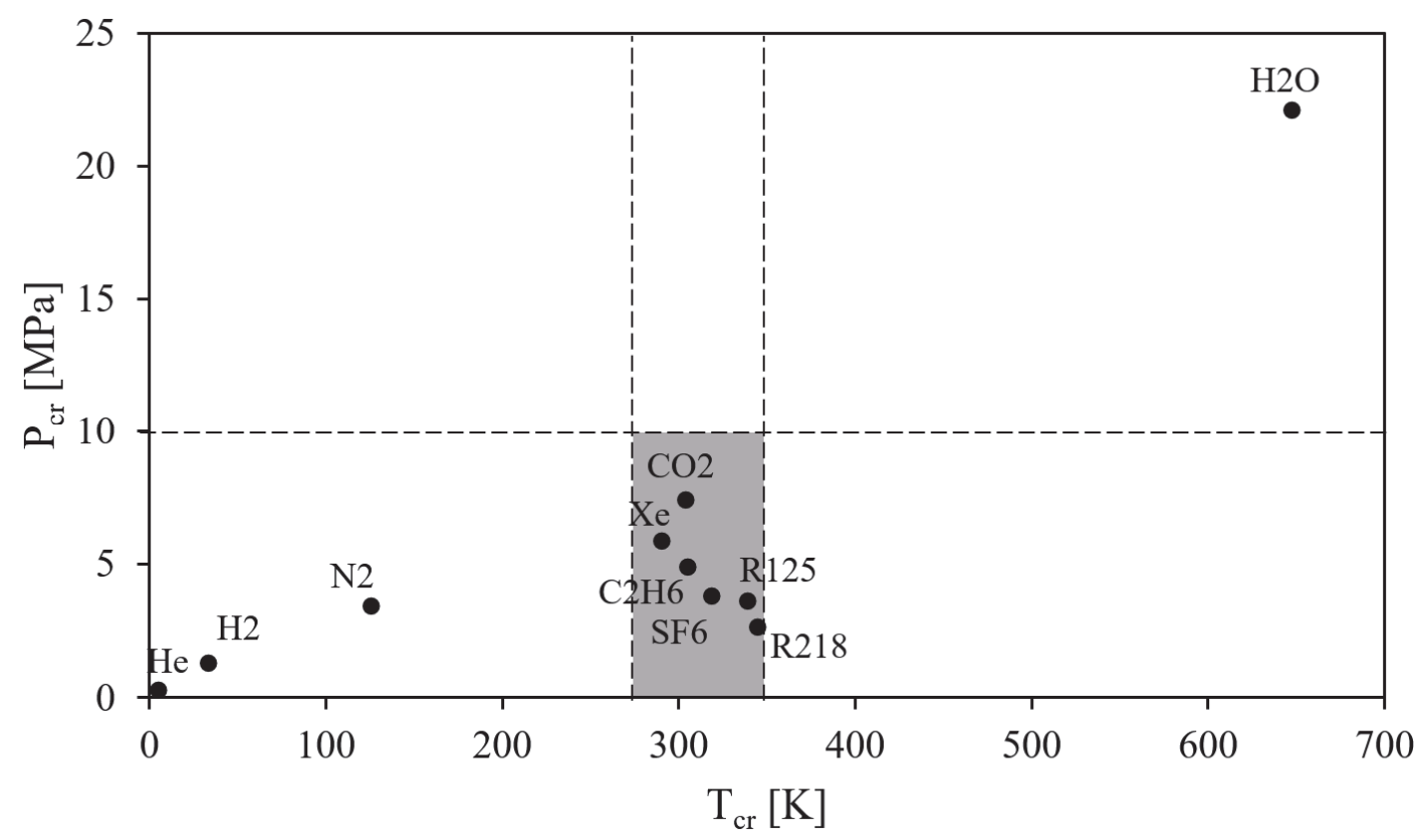

Figure 2.31. Classification of fluids as a function of its critical temperature, $T_{c r}$, and critical pressure, $P_{c r}$

Working fluids such as helium (He) have been previously studied for its use in Brayton cycles (Schleicher, Raffray and Wong, 2001). However, these fluids have a critical temperature below $275 \mathrm{~K}$, which would require a heat sink with a temperature under the environmental one in order to make use of the pericritical concept. Thus, these fluids are not suitable for pericritical cycles. On the other hand, fluids such as water are not valid either due to its too high critical temperature. The compressor would work far away from the critical point.

Pericritical cycles have shown the benefits of increasing the high-side pressure. Thus, moderate pressures will be preferred as the higher the pressure, the more expensive the system will be. Although there is not a strict limitation with the critical pressure, a value of 10 $\mathrm{MPa}$ is selected as limit in Figure 2.31 in order to remind that the critical pressure must also be considered.

The fluids fulfilling the limitations of critical temperature and pressure will be candidates for working in pericritical cycles. Figure 2.31 shows in grey the design window and some of the fluids inside it. Appendix A contains more fluids found in this design window. Ethane $\left(\mathrm{C}_{2} \mathrm{H}_{6}\right)$, 
sulfur hexafluoride $\left(\mathrm{SF}_{6}\right)$ and some refrigerants such as $\mathrm{R} 125$ are some examples of these candidate fluids. Even a mixture of some of these fluids could be a possibility for this type of cycles (Invernizzi, 2017). However, these substances will have to deal with the important constraint of thermochemical stability at the operating temperatures required by the cycle. For example, the maximum safe temperatures of organic compounds are around $400-450{ }^{\circ} \mathrm{C}$ (Invernizzi, 2017).

Carbon dioxide $\left(\mathrm{CO}_{2}\right)$ is the working fluid commonly used in supercritical Brayton cycles (then called supercritical carbon dioxide, $\mathrm{s}-\mathrm{CO}_{2}$ ) because of its stability, relative inertness, non-toxicity, abundance and low cost (Dostal, 2004). Since the first proposal of these cycles by Feher (1968), this fluid has gained importance because it can withstand high temperatures and reach high efficiencies (Dunham and Iverson, 2014; Turchi et al., 2012). The disadvantage of $\mathrm{CO}_{2}$ with respect to the other candidate fluids shown in the design window of Figure 2.31 is the high critical pressure. A lower critical pressure means that the cycle can take advantage of higher reduced pressures at lower pressures, which can be an advantage in terms of costs. However, the main limit of these fluids with lower critical pressure is usually the maximum temperature that they can withstand, which limits the maximum cycle efficiency.

This chapter has analysed pericritical cycles with the thermodynamic properties of $\mathrm{CO}_{2}$. Nonetheless, the systematic characterization carried out in this chapter could be extrapolated to other candidate fluids. The relative position of the compressor with respect to the discontinuity line marks the main trends of the cycle behavior. Therefore, although the absolute values will depend on the fluid, the main trends marked by the discontinuity line will be similar.

As an example, the results obtained with $\mathrm{CO}_{2}$ will be compared with one of the candidate fluids shown in Figure 2.31: R125. These two fluids have very different thermophysical properties. For example, the value of the $c_{p} / R$ factor measured at ideal gas conditions is 4.5 for $\mathrm{CO}_{2}$ and 12.27 for R125. This factor has a high influence on the biphasic bell shape in h-P and T-s diagrams (Rovira et al., 2013) and, therefore, it will have a high influence in the shape of the whole discontinuity line. Figure 2.32 shows the differences between the discontinuity lines of $\mathrm{CO}_{2}$ and $\mathrm{R} 125$. The reduced enthalpy, $h r$, has been set to zero in the critical point $\left(P_{r}=1\right.$ and $\left.T_{r}=1\right)$ in order to better appreciate the differences. 


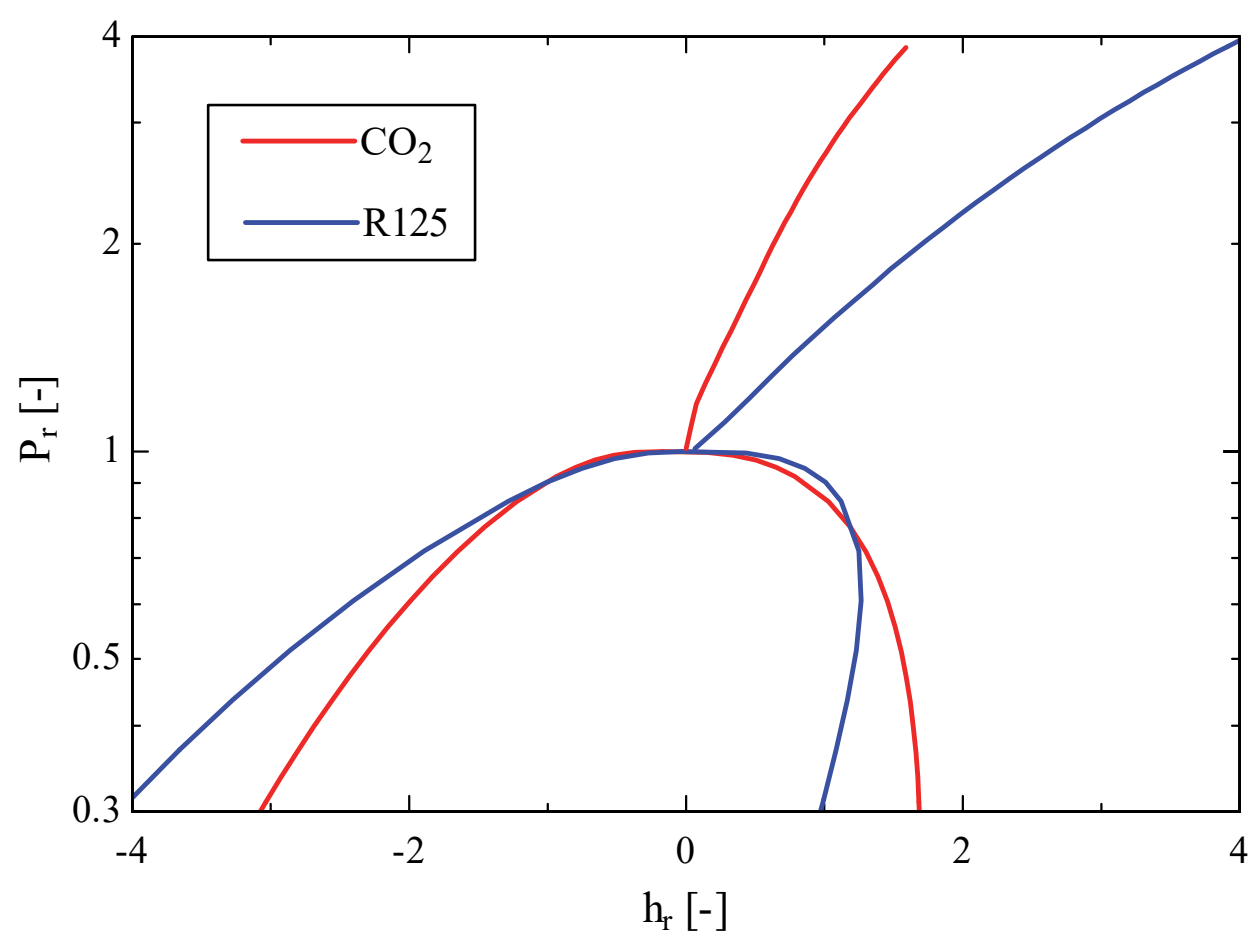

Figure 2.32. Discontinuity line of $\mathrm{CO}_{2}$ and $\mathrm{R} 125$ in $\mathrm{P}-\mathrm{h}$ diagram

The big difference in $c_{p} / R$ factor (and therefore in the discontinuity line shape) between $\mathrm{CO}_{2}$ and R125 involves different cycle behaviors (Rovira et al., 2013). Figure 2.33 shows the thermal efficiency, $\eta_{t h}$, of pericritical cycles with $\mathrm{CO}_{2}$ and $\mathrm{R} 125$ as working fluid. The reduced variables are high-side pressure, $P_{H, r}$, and compressor inlet temperature, $T_{c, i n}$.

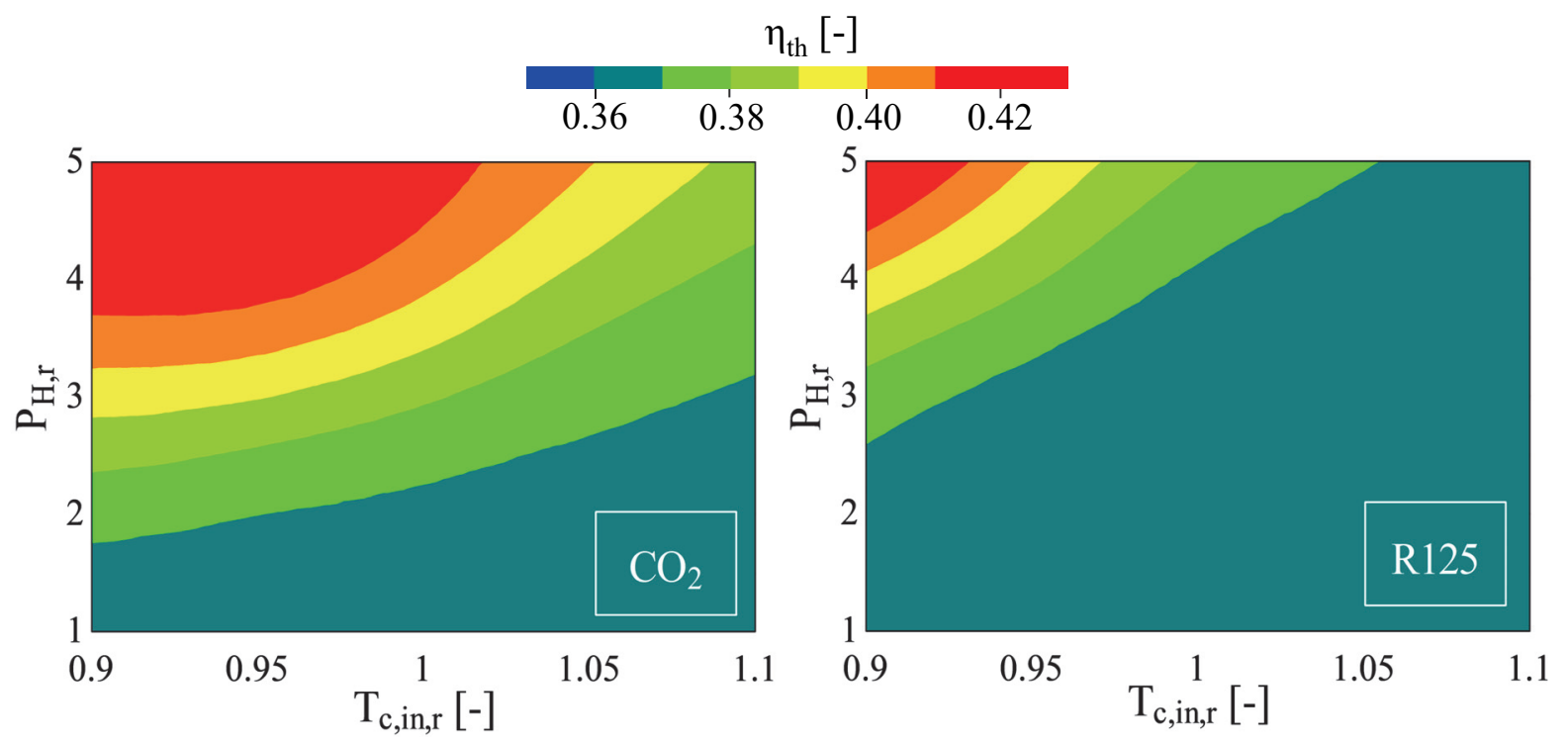

Figure 2.33. Thermal efficiency, $\eta_{t h}$, of pericritical cycles with $\mathrm{CO}_{2}$ and $\mathrm{R} 125$ as working fluid as a function of the high-side pressure, $P_{H, r}$, and compressor inlet temperature, $T_{c, i n, r}$ 
Although the efficiency values are different in $\mathrm{CO}_{2}$ and $\mathrm{R} 125$, the trends marked by the discontinuity line are similar. Cycles with high-side pressure below the discontinuity line or above but close to it have similar efficiencies $\left(\eta_{t h},=[0.36,0.37]\right)$. These cycles have the compressor working at ideal gas conditions. When the distance between the high-side pressure and the discontinuity line is big enough, raising pressure and lowering compressor inlet temperature involve higher thermal efficiencies. In the case of R125, higher pressures and lower compressor inlet temperatures are needed to increase the efficiency, but anyway the trends are similar than in $\mathrm{CO}_{2}$. Absolute values will change from a fluid to the other, but the main trends established by the discontinuity line will remain.

\subsection{Summary}

This chapter presents a comprehensive and systematic analysis of regenerative closed cycles working in the thermodynamic region around the critical point. This region is called pericritical and the cycles working in this region can do it totally or partially at supercritical conditions, agglutinating in the analysis the cycles commonly known as supercritical and transcritical. This analysis allows to generalize the characterization of cycles working around the critical point.

A new thermodynamic coefficient, called logarithmic factor of isobaric expansion, is especially relevant during the thermodynamic characterization of pericritical cycles. The points with maximum value of this dimensionless coefficient define the discontinuity line. This line separates the cycles in those with the compressor working in the liquid region and those with the compressor working in the gaseous region. The advantage of the liquid region is the low compression power required and the disadvantage the additional heat needed in the cycle due to the limitations in the regenerative phase. In the gaseous region occurs the opposite: the advantage of the better performance of the regenerative phase comes at the expense of greater compression powers. The balance between the compression work and the limitations in the heat regeneration phase characterizes the thermal efficiency and the optimum pressure ratio to achieve it.

The cycles analysed have all the same Carnot efficiency. In this way, all of them have the same potential. The low-side pressure is optimized to maximize the thermal efficiency for different compressor inlet temperatures and high-side pressures. When the high-side pressure is close to or below the pressure corresponding to the compressor inlet temperature in the 
discontinuity line, the optimum compressor position works in the ideal gas region and the thermal efficiency is the same regardless of the compressor inlet temperatures and the highside pressure. However, compressor inlet temperature and high-side pressure are highly related when the high-side pressure is raised because the optimum low-side pressure approaches the compressor inlet to the discontinuity line. The thermal efficiency increases when the high-side pressure is raised at least above 2.2 times the pressure corresponding to the compressor inlet temperature in the discontinuity line. For these cases, cycles with lower compressor inlet temperature can achieve higher efficiencies due to the larger distance between the high-side pressure and the discontinuity line.

The inefficiencies of the different phases of pericritical cycles have a great impact on the thermal efficiency and on the optimum pressure ratio needed to maximize it. Cycles with small regenerator effectiveness and high turbomachines efficiency achieve its maximum performance at large pressure ratios in the gaseous region. The optimum pressure ratio decreases when the regenerator effectiveness increases and the turbomachine efficiency decreases. For cycles with high effectiveness and low turbomachine efficiency, the optimum pressure ratio is the one with the low-side pressure touching the discontinuity line.

The purpose of this chapter has been to generalize the analysis of the different thermodynamic regions around the critical point instead of divining the analyses in transcritical and supercritical cycles. The result has been the identification of different cycle behaviors depending on the compressor position in the thermodynamic diagram. This knowledge helps to identify the best thermodynamic region for a cycle with specific conditions.

The results shown in this chapter have been obtained with the equation of state of $\mathrm{CO}_{2}$. However, this analysis could be extrapolated to other fluids for which, while the numerical values would be different, the trends would be similar. 


\section{The Multi-HeAting CyClE}

The compressor position in the thermodynamic diagram rules the behavior of pericritical cycles. Pericritical cycles with the compressor working in the liquid region (or close to it) require particularly low compression power. However, this low compression power comes at the expense of additional heat needed by the cycle due to a mismatch between the specific heats in the regeneration phase. While a small specific work increases cycle performance, the increment of heat needed decreases it. The weight of each of these two terms in the efficiency must be carefully analysed in pericritical cycles.

This chapter presents a new layout to reduce the mismatch of specific heats in the regenerator, and therefore, increase the cycle performance. This layout consists in supplying additional heats to the high-pressure side during the heat regeneration. These additional heats replace part of the heat supplied by the primary heat source and may be supplied by secondary heat sources at lower temperatures. The lower exergetic cost of the heats at lower temperature increases the exergy efficiency of the cycle. This new layout with additional heats supplied at lower temperatures will be called multi-heating cycle. This chapter will describe different possibilities for this new layout, and the discontinuity line will help to characterize the optimum configuration depending on the limiting temperatures, pressures and inefficiencies.

\subsection{Multi-heating concept}

A large part of the heat needed in pericritical cycles with the compressor working in the liquid region (or close to it) comes from the specific heat imbalance between the high- and low- 
pressure sides. Previous chapter showed that this imbalance is dragged along the regeneration, resulting in a regeneration heat loss $\Delta h_{a}$ that must be supplied by the heat source (see Equation 2.67). However, this imbalance would not have to be dragged if it were solved when it happens. The way of doing this is by supplying additional heat to compensate the regeneration enthalpy loss in each infinite part of the high-pressure side of the regenerator. The result would be that infinite additional heats, $q_{a h, i}$, at different temperatures, $T_{i}$, compensate the total regeneration heat loss, $\Delta h_{a}$, along the regenerator. Equation 3.1 expresses this idea, showing the value of each heat, $q_{a h, i}$, to compensate the specific heat difference at each temperature.

$$
\Delta h_{a}=q_{a h, 1}+\cdots+q_{a h, \infty}=\int_{T_{m}}^{T_{m}+d T} \Delta c_{P, 1} d T+\cdots+\int_{T_{t, o u t}-d T}^{T_{t, o u t}} \Delta c_{P, t, \text { out }} d T
$$

The infinite additional heats, $q_{a h, i}$, are supplied from the pinch point temperature, $T_{m}$, to the outlet turbine temperature, $T_{t, \text { out }}$.

Figure 3.1 shows a scheme in P-h and T-s diagrams of a pericritical cycle with the proposed additional heats during the regeneration. The small arrows represent the infinite additional heats supplied after the pinch point (indicated by the dashed blue line corresponding to $T_{m}$ ), and the big arrow the primary heat. The red dashed lines indicate the heat regeneration limits when the infinite additional heats are supplied. The regeneration limit to the right is increased in comparison to the simple regeneration (black dashed line), which involves a reduction of the primary heat. The regeneration limit to the left is common in cycles with and without additional heats.
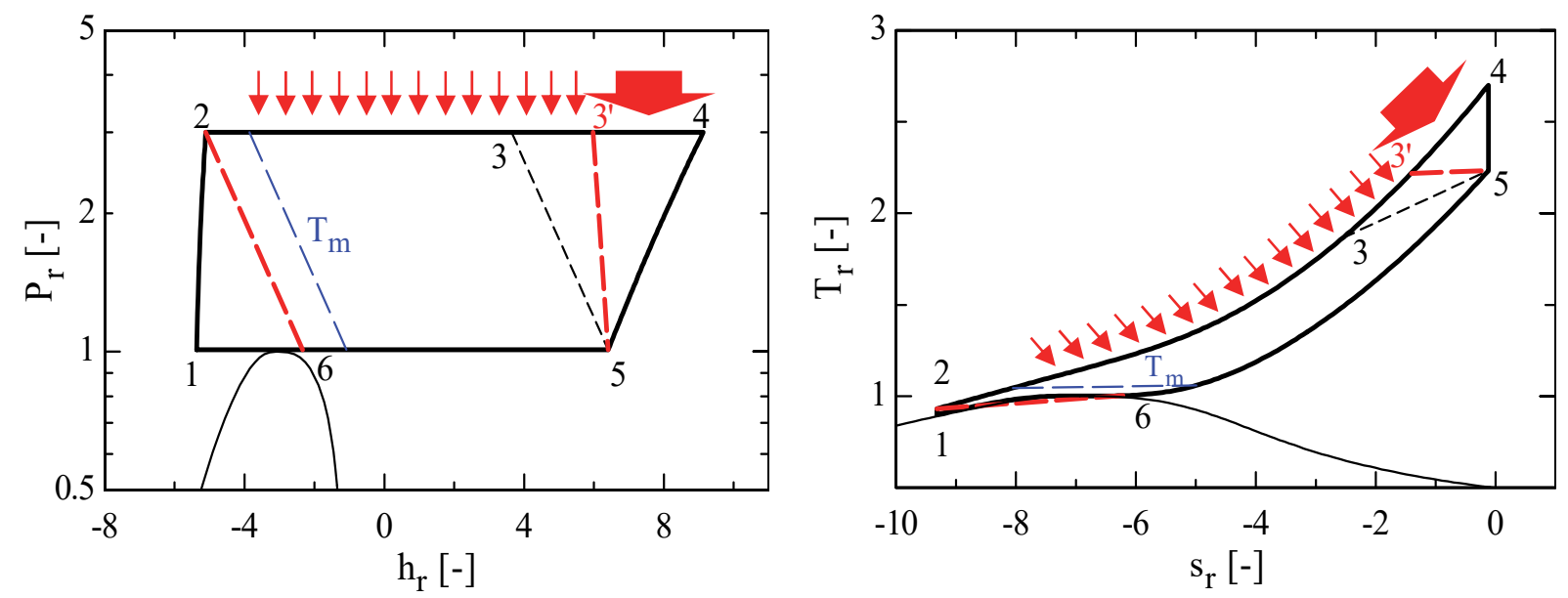

Figure 3.1. Scheme of a pericritical cycle with infinite additional heats supplied during the regeneration in diagrams $\mathrm{P}-\mathrm{h}$ (left) and T-s (right) 
The value of each additional heat depends on the specific heat difference between the low and high-pressure isobars. Figure 3.2 shows an example of the additional heats, $q_{a h, i}$, needed during the regeneration of a pericritical cycle at the conditions of Table 3.1. This heat is null at the pinch point temperature $T_{m}$ and increases rapidly towards a maximum. This maximum corresponds to the point with the highest difference of enthalpy increment between two isothermal lines. The additional heat required to balance the intermediate heat exchanger decreases after this maximum to values close to zero. It would get to zero at ideal conditions, where isothermal and isenthalpic lines are the same. In the cycles studied in this work, the turbine works close to ideal conditions, and therefore the additional heat required at turbine outlet temperature will be close to zero.

Table 3.1. Parameters used to analyze the regeneration in a pericritical cycle

\begin{tabular}{ll}
\hline Parameter & Value \\
\hline Reduced high-side pressure, $P_{H, r}$ & 3 \\
Reduced low-side pressure, $P_{L, r}$ & 1 \\
Reduced compressor inlet temperature, $T_{c, \text { in }, r}$ & 0.9 \\
Carnot factor, $\tau=T_{t, \text { in }} / T_{c, \text { in }}$ & 3 \\
\hline
\end{tabular}

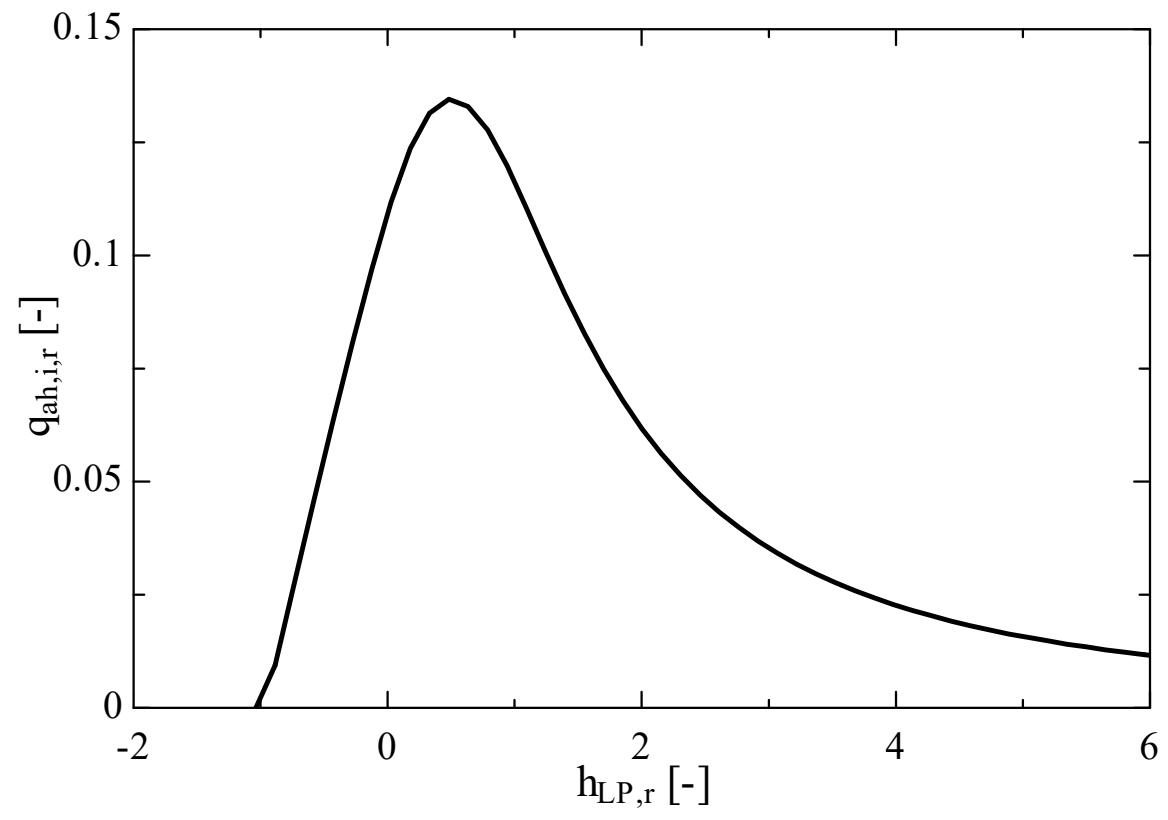

Figure 3.2. Additional heats, $q_{a h, i}$, needed along the regeneration of a pericritical cycle in order to balance the intermediate heat exchanger 
The resulting temperatures during the regeneration with infinite additional heats are shown in red in Figure 3.3. The temperatures of the high-pressure side, $T_{H P}$, are compared with the temperatures obtained in a simple regeneration without additional heats (shown in black). The temperatures of the low-pressure side, $T_{L P}$, are the same in both cases because the temperatures are represented as a function of the low-pressure enthalpy and there is no modification in this part of the cycle. However, the temperatures at the high-pressure side are different when the additional heat is added after $T_{m}$. The additional heats achieve to balance the regeneration making parallel the temperature lines of the high and low-pressure sides.

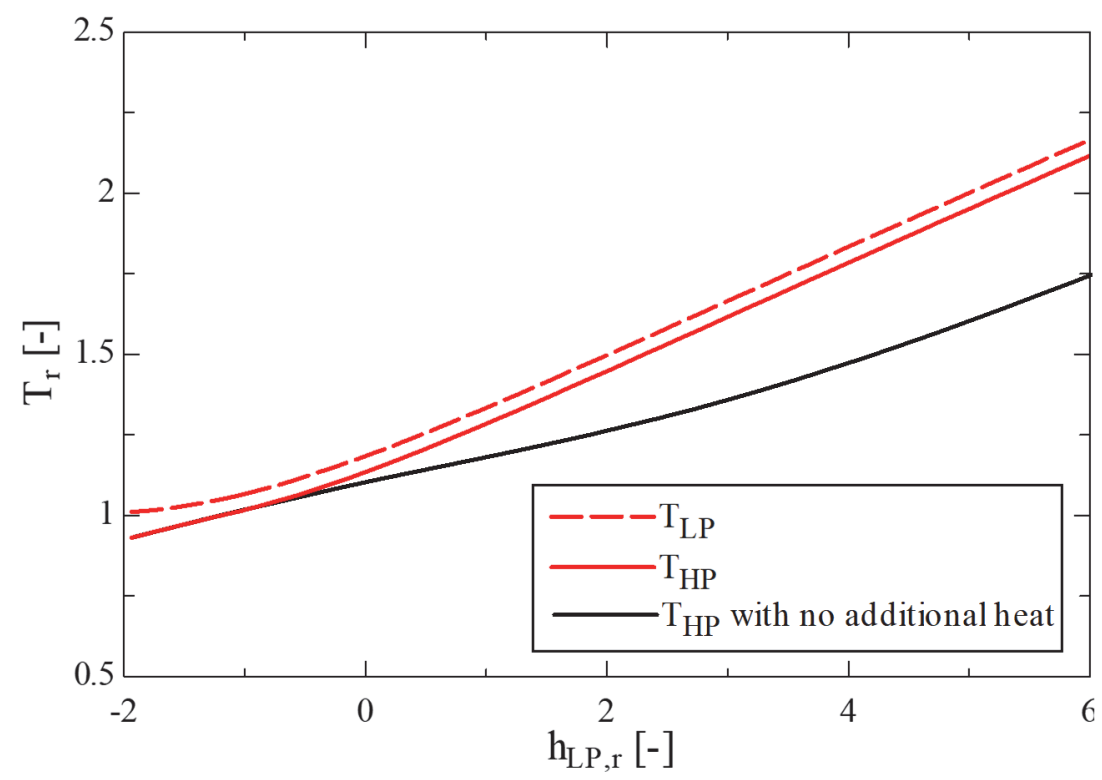

Figure 3.3. Temperatures of the high-pressure side, $T_{H P}$, and low-pressure side, $T_{H P}$, along the regenerator of a pericritical cycle with infinite additional heats supplied during the regeneration (in red), and without additional heats (in black).

Note that Equation 3.1 defines the additional heats needed to balance an ideal regeneration. This means that the resulting temperatures in the high and low-pressure sides would be the same. Temperatures in Figure 3.3 are calculated to have a minimum temperature difference between both pressure sides in order to facilitate the understanding.

In the case of the simple regenerative cycle (i.e., without additional heats), the temperature lines are almost parallel for enthalpies above $h_{L P, r}=3$. There is almost no imbalance at these enthalpies because the specific heats are similar in both pressure sides. Therefore, if additional heats were supplied up to low-pressure enthalpies $h_{L P, r}=3$, instead of up to the turbine outlet temperature, the primary heat would still be significantly reduced. Equation 3.2 expresses the additional heat supplied along the first part of the regeneration up to a temperature, $T_{a h}$. 


$$
q_{a h}=\int_{T_{m}}^{T_{m}+d T} \Delta c_{P, 1} d T+\cdots+\int_{T_{a h}-d T}^{T_{a h}} \Delta c_{P, a h} d T
$$

Figure 3.4 represents in red the amount of additional heat, $q_{a h}$, supplied along the regenerator up to a temperature, $T_{a h}$. The maximum allowable value of this temperature $T_{a h}$ is the turbine outlet temperature, $T_{t, \text { out }, r}=2.2$. In this case, tll the regeneration heat loss $\Delta h_{a}$ is supplied with infinite heats at temperature from $T_{m}$ to $T_{a h, r}=T_{t, \text { out }}=2.2$. The amount of additional heat supplied, $q_{a h}$, is smaller when the temperature $T_{a h}$ decreases. Nonetheless, supplying additional heats up to temperatures above $T_{a h, r}=1.6$ still compensates a big part of the regeneration heat loss $\Delta h_{a}$. For example, the amount of additional heat, $q_{a h}$, supplied at temperature $T_{a h, r}=1.6$ is $79 \%$ of the maximum possible amount, $\Delta h_{a}$.

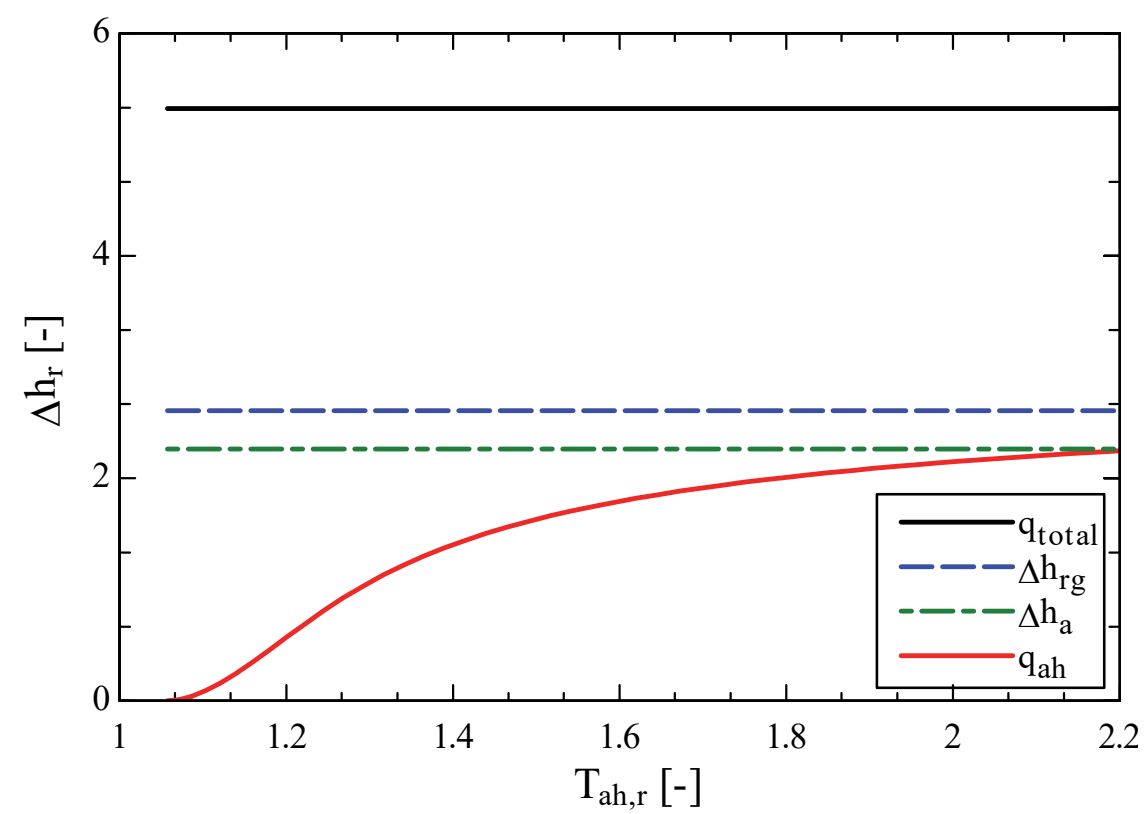

Figure 3.4. Additional heat, $q_{a h}$, supplied during the regeneration by means of infinite heats supplied up to a temperature, $T_{a h}$, in order to reduce the regeneration heat losses $h_{a}$ and $h_{r g}$, in a cycle that needs a total heat, $q_{\text {total }}$.

The total heat added to the cycle, $q_{\text {total }}$, will be constant regardless the additional heat supplied. This means that the sum of the primary heat, $q_{p h}$, and the additional heat, $q_{a h}$, is constant:

$$
q_{\text {total }}=q_{p h}+q_{a h}
$$

Supplying additional heats up to a temperature $T_{a h, r}=1.6$ represents $34 \%$ of the total heat, $q_{\text {total. }}$. This implies that $34 \%$ of the heat is added at temperatures much lower than the turbine inlet temperature. 
If the total heat supplied to the cycle and the net specific work remain constant, the thermal efficiency will also do it. Thus, the proposed method to reduce the heat at high temperature does not improve the thermal efficiency. The advantage of these cycles with multi-heating is the reduction of the average source temperature, and thermal efficiency does not take this into account. This advantage must be analysed by means of an exergy analysis. The exergy will measure the costs of the heats, and the exergy efficiency will determine the cycle performance.

\subsection{Multi-heating layouts}

The use of infinite additional heats serves as theoretical explanation about how to balance the regenerator in pericritical cycles. However, this would be impracticable in a real cycle. Two real layouts to balance the regenerator by means of multi-heating are proposed and analysed in this section. The first layout consists in supplying heat to the high-pressure side of the regenerator in a continuous manner through another fluid. This involves the use of a threeflow intermediate heat exchanger. Two of the flows correspond to the fluid in the high- and low-pressure isobars and the third one is an additional hot fluid. The additional fluid could be heated by the primary heat source or by a different one. In the second layout, the regenerative phase is divided at least into two parts with a heat in-between supplied to the high-pressure side by an external heat source. This layout is a discrete method to balance the heat exchanger in steps instead of doing it in a continuous manner.

\subsubsection{The three-flow pattern}

The layout consisting in supplying heat in a continuous manner will be called the three-flow pattern. Figure 3.5 shows a diagram of a pericritical cycle with this layout. The high-pressure flow exiting the compressor goes through a first regenerator to exchange heat with the lowpressure side about to enter the precooler. The purpose of this first regenerator is to contain the pinch point and make sure that the upcoming additional heat is supplied once the pinch point has passed. This regenerator can be omitted if the pinch point occurs at the compressor outlet. The additional heat supplied by the three-flow pattern comes after the first regeneration. The additional heat is supplied in a heat exchanger with a three-flow pattern to balance the regeneration. 


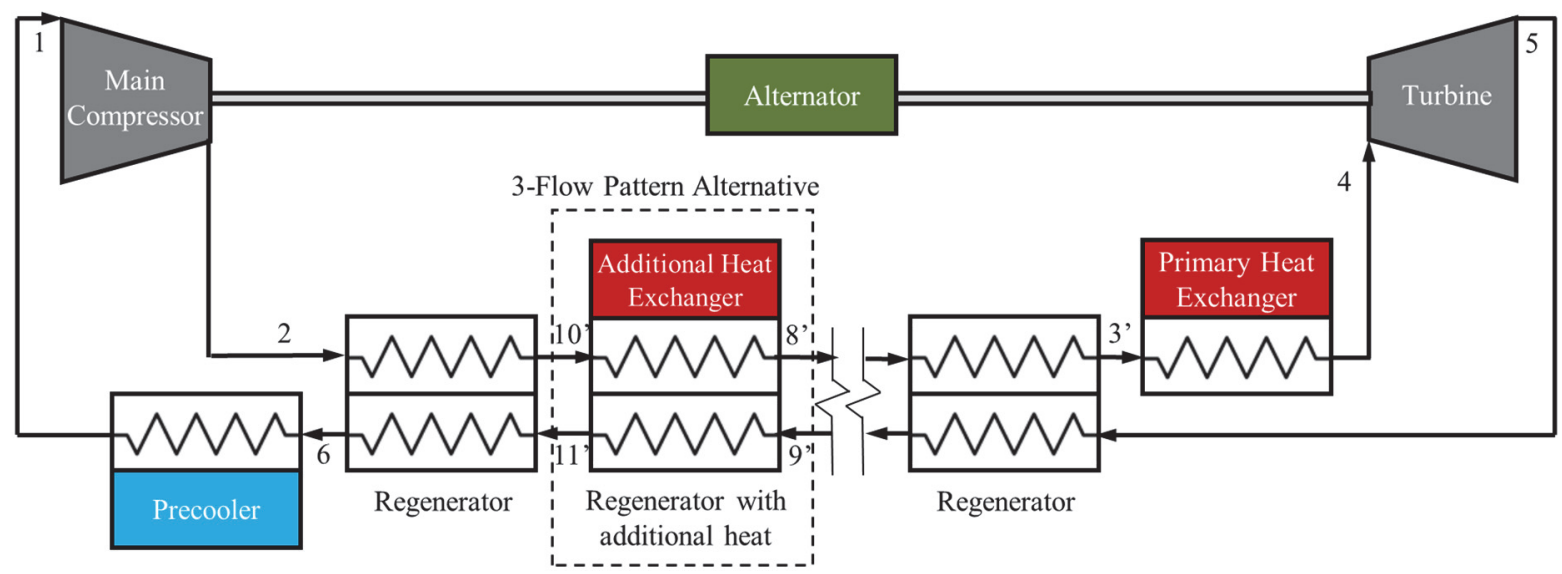

Figure 3.5. Diagram of a regenerative pericritical cycle with the three-flow pattern layout

(diagram design inspired by Dyreby (2014))

Figure 3.6 shows two possible embodiments of the three-flow heat exchanger. A third fluid supplies the additional heat, $a h$, to the cycle: either to the high-pressure fluid, $H P$, or to the low-pressure fluid, $L P$. The arrows indicate the direction of the heat transfer.

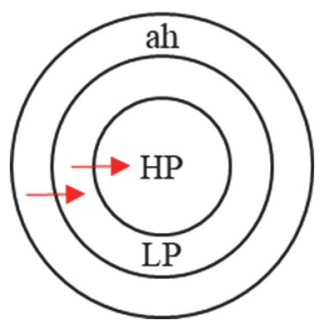

(a)

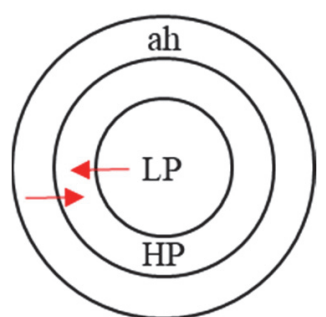

(b)

Figure 3.6. Possible embodiments of the three-flow heat exchanger. The fluid supplying the additional heat, $a h$, is in the outer part and the low-pressure, $L P$, and high-pressure, $H P$, fluids in the inner part.

The third fluid supplies additional heat up to a certain temperature corresponding with point 8 ' in Figure 3.5. More additional heats could be supplied at higher temperatures afterwards. In this way, the gap shown in Figure 3.5 could be filled with more dashed-line boxes. Each three-flow pattern added to the cycle provides the possibility of supplying the additional heat by means of a different heat source. If the temperature of the last three-flow pattern layout is not the turbine outlet temperature then a last regenerator is needed before the primary heat exchanger, as shown in Figure 3.5.

Figure 3.7 shows the P-h and T-s diagrams of a pericritical cycle with three-flow pattern layout. The red dashed lines indicate the heat regeneration limits in a cycle with the threeflow pattern layout, and the black dashed line the limits in a simple cycle without additional 
heats. The left part of the regeneration (line 2-6) is common in both cases. However, the right part is different. The line 3'-5 indicates that the primary heat of the cycle with multi-heating $\left(h_{4}-h_{3}\right)$ is smaller than the heat of the simple cycle $\left(h_{4}-h_{3}\right)$.
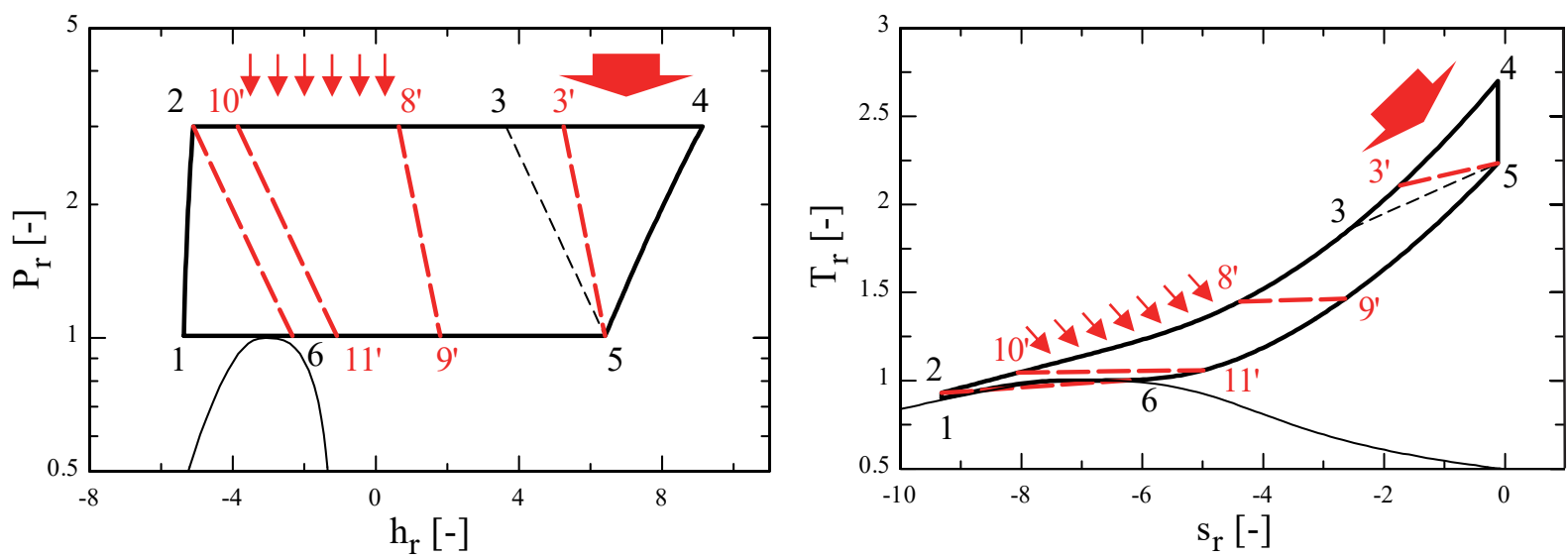

Figure 3.7. Scheme of a pericritical cycle with the three-flow pattern layout in diagrams P-h (left) and T-s (right)

An example of the resulting temperatures during the regeneration with the three-flow pattern layout is shown in red in Figure 3.8. These temperatures are compared with the temperatures obtained in a simple regeneration without additional heats (in black).

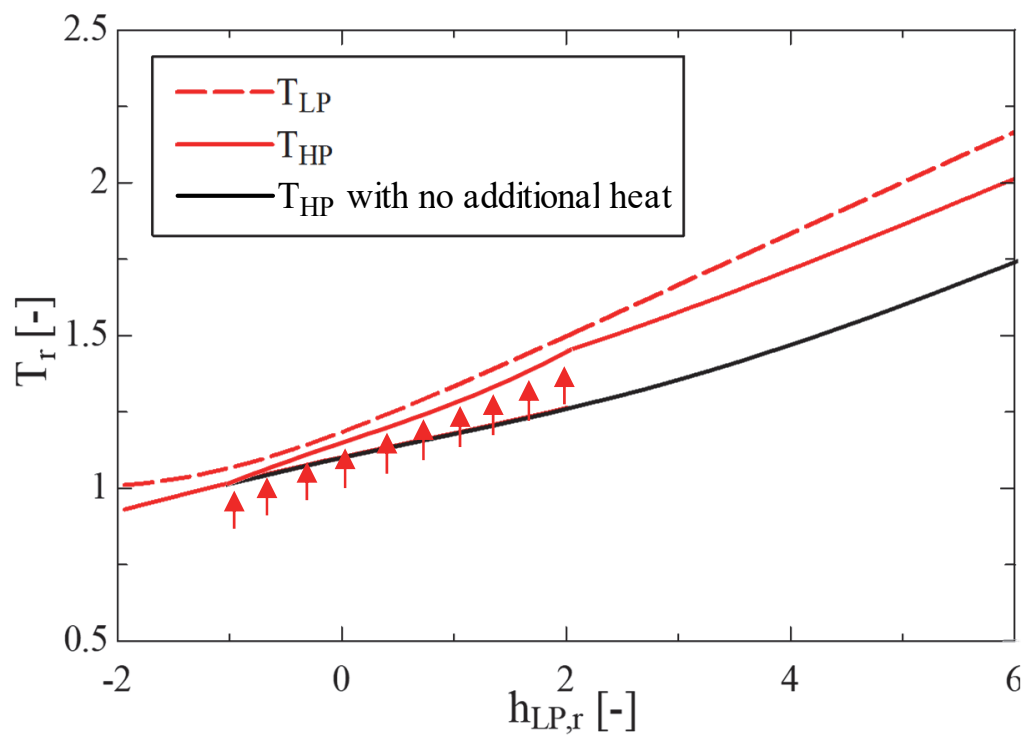

Figure 3.8. Temperatures of the high-pressure side, $T_{H P}$, and low-pressure side, $T_{H P}$, along the regenerator of a pericritical cycle with three-flow pattern layout (in red), and without additional heats (in black).

The low-pressure temperature, $T_{L P}$, is the same in both cases because the low-pressure side is not modified. On the other hand, it can be observed that, after the pinch point temperature $T_{m}$, 
when the additional heat is added, the high-pressure temperatures are almost parallel to the low-pressure one. Once the flows are in the last regenerator, the temperatures direction starts to diverge again.

The temperatures shown in Figure 3.8 are those corresponding to the high and low-pressure sides of the cycle. However, there is an extra fluid in three-flow heat exchanger, which can be different from the fluid of the cycle. The mass flow rate and the unbalance of specific heats of the three fluids will establish the shape of the high-pressure temperature.

\subsubsection{Multi-step dual heating}

While the three-flow pattern layout supplies the additional heat during the regeneration, the multi-step dual heating divides the process into two steps: heat supply and regeneration. Figure 3.9 shows the diagram of regenerative pericritical cycle with the step dual heating layout. The dashed-line box contains the new layout to balance the cycle regeneration: first, a regeneration, and second, a heat supply to the high-pressure side.

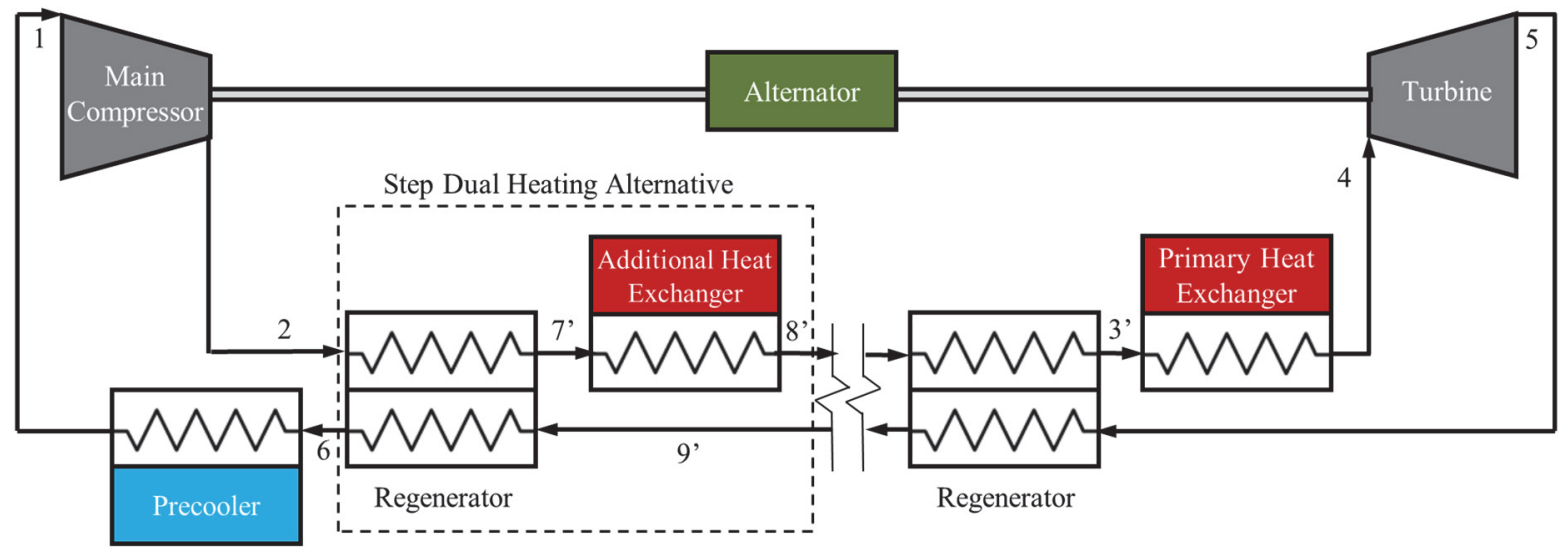

Figure 3.9. Diagram of a regenerative pericritical cycle with the step dual heating layout (diagram design inspired by Dyreby (2014))

The gap after point $8^{\prime}$ means the same than in the previous case: there can be more than one dashed-line box in the cycle. Therefore, different heat steps at different temperature could substitute part of the primary heat. The regenerator of the first step dual heating covers the part where the pinch point may appear. Thus, there is no need of a regenerator previous to the dashed-line box, as it happened in the diagram of the cycle with the three-flow pattern in Figure 3.5.

Figure 3.10 shows the P-h and T-s diagrams of a regenerative pericritical cycle with step dual heating layout. The red dashed lines indicate the heat regeneration limits in a cycle with step 
dual heating, and the black dashed line in a simple cycle without additional heat. The lines limiting the two regenerators in the P-h diagram are parallel due to the energy balance. Therefore, after the additional-heat supply $\left(h_{8},-h_{7}\right)$, the regeneration limiting lines 8 '-9 and 3'-5 are more vertical, allowing higher temperatures in the high-pressure side of the regenerator. Thus, the primary heat line in a cycle with one step dual heating $\left(h_{4}-h_{3}{ }^{\prime}\right)$ is reduced.
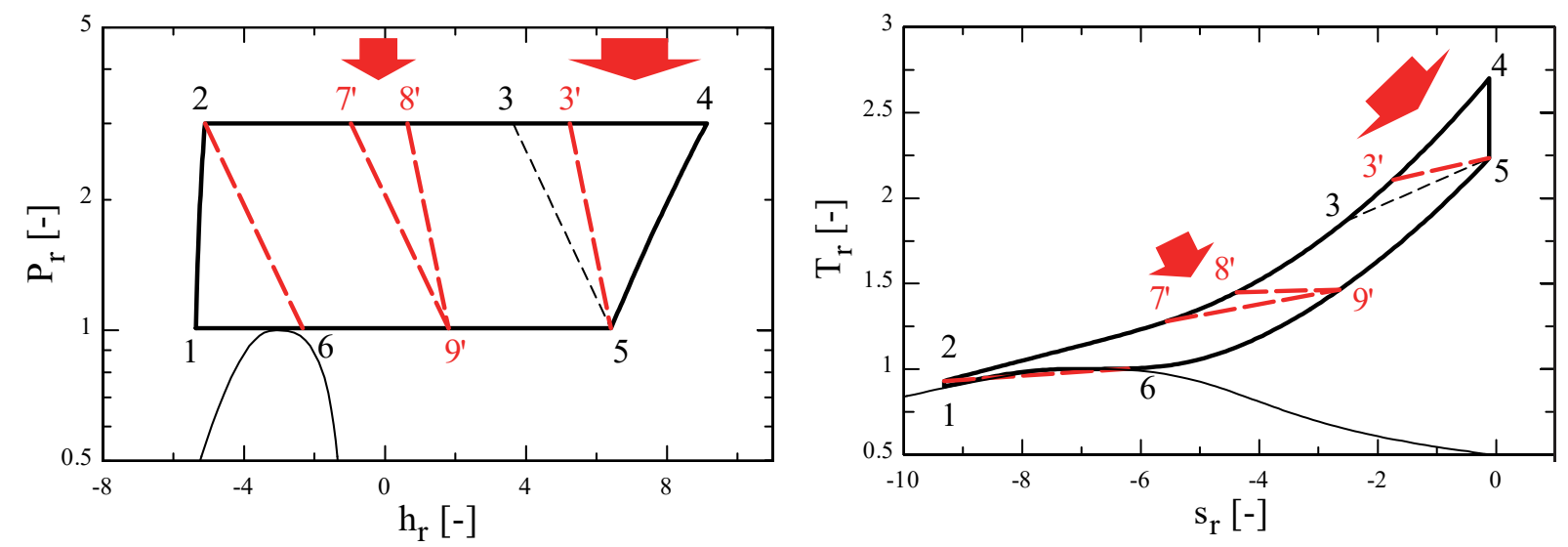

Figure 3.10. Scheme of a pericritical cycle with the step dual heating layout in diagrams P-h (left) and T-s (right)

The temperatures of both sides of the regenerator are presented in Figure 3.11. The highpressure temperatures of a cycle with one step dual heating (in red) are compared with the temperatures obtained in a simple regeneration without additional heats (in black).

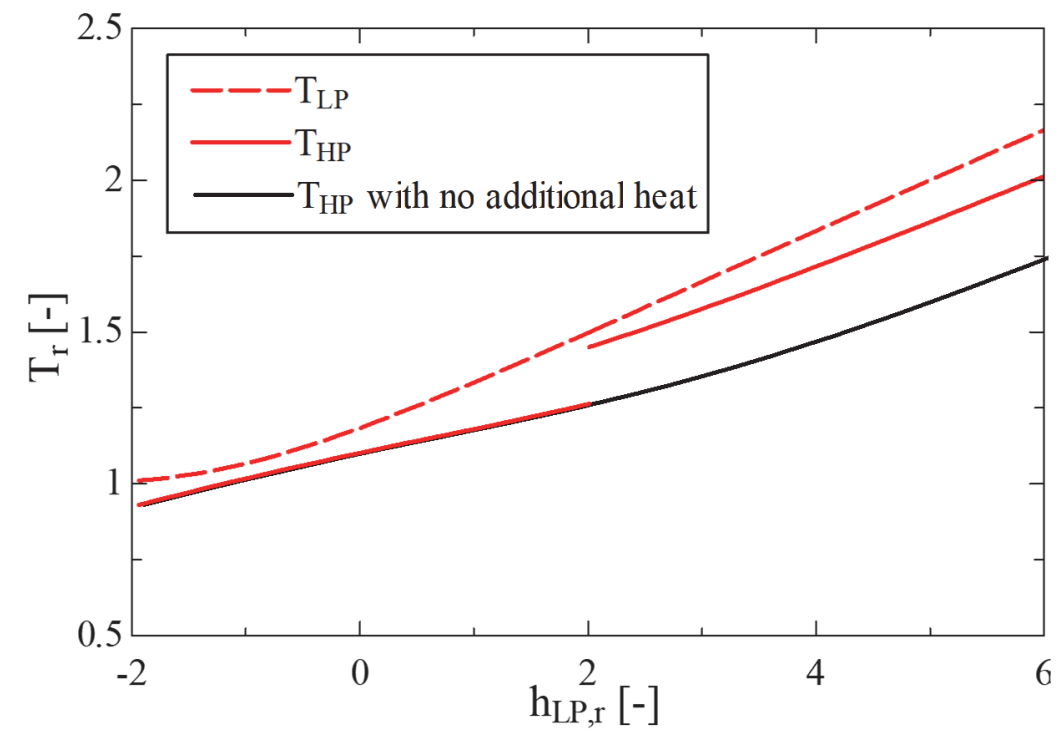

Figure 3.11. Temperatures of the high-pressure side, $T_{H P}$, and low-pressure side, $T_{H P}$, along the regenerator of a pericritical cycle with the step dual heating layout (in red), and without additional heats (in black). 
The low-pressure temperature, $T_{L P}$, is the same in both cases because the low-pressure side is not modified. The additional heat involves a jump in the shape of the high-pressure temperature, $T_{H P}$. This jump is what causes a greater temperature in the last part of the highpressure. In this way, the primary heat is reduced in the cycle with step dual heating layout.

\subsection{Analysis of multi-heating cycles}

\subsubsection{Energy analysis}

Three-flow pattern and multi-step dual heating are two different layouts proposed for multiheating cycles. The three-flow pattern supplies the heat in a continuous manner and the multistep dual heating does it in steps. However, both have in common that, if the maximum temperature of the additional heat and its corresponding regeneration are the same, the value of the additional heat is the same. This can be understood looking at the P-h and T-s diagrams from Figure 3.7 and Figure 3.10. The isothermal line corresponding to the maximum temperature of the additional heat is the line $8^{\prime}-9^{\prime}$ and the regenerated heat, $\Delta h_{\text {reg, }}$, is

$$
\Delta h_{r e g}=h_{91}-h_{6}
$$

Thus, if the maximum temperature of the additional heat and the regenerated heat, $\Delta h_{\text {reg, }}$ are the same, the additional heat, $q_{a h}$, will be in both cases:

$$
q_{a h}=\left(h_{8 \prime}-h_{2}\right)-\Delta h_{r e g}
$$

Note that this is true assuming that both layouts of multi-heating are applied in a cycle with the same points 1245 .

The additional heat from Equation 3.5 is easily appreciated in the P-h diagram with multi-step dual heating of Figure 3.10, where the value of the additional heat is the enthalpy difference between points 7' and 8'. However, this heat is hidden in the high-pressure side in the P-h diagram with the three-flow pattern layout from Figure 3.7. The multi-step dual heating layout is easier to deal with because regeneration and heat addition can be defined separately. This work will use the scheme of multi-step dual heating to define the cycles with additional heats. Nevertheless, the results obtained will be valid for both three-flow pattern and multistep dual heating layouts unless otherwise specified. In order to call both layouts with a common name, these cycles are called multi-heating cycles. 
The total heat supplied to the cycle will depend on the capacity to recuperate heat of the intermediate heat exchangers. Previous chapter defined this capacity by means of the intermediate heat exchanger effectiveness $\varepsilon$ (see Equation 2.84). This effectiveness will also be used to define the global regeneration phase in multi-heating cycles. This global effectiveness defines the regeneration of the low-pressure side, independently of what happens in the high-pressure side. This allows the comparison between simple regenerative cycles and cycles with modifications inside the cycle, such as the proposed multi-heating cycles, focusing only on what happens inside the regeneration.

The idea of defining a global effectiveness has been previously used by Padilla et al. (2015a) in cycles where there is more than one regenerator. However, Padilla et al. define the global effectiveness in a more specific way than the one used in previous chapter, and this could lead to wrong interpretations in some of the cases analysed in this work. Padilla et al. define the global effectiveness as:

$$
\varepsilon=\frac{h_{5}-h_{6}}{h_{5}-h_{6} @ T_{2}}
$$

where $h_{6 @ T_{2}}$ is the enthalpy at state 6 in Figure 3.10 calculated based on the minimum temperature that the hot stream leaving the regenerator could reach, $T_{2}$. In this way, the denominator pretends to define the maximum heat that an infinite heat exchanger could exchange by assuming that the minimum temperature that the hot stream leaving the regenerator could reach is $T_{2}$. However, it was seen in Figure 2.15 that, in some cases, this temperature could be greater than the compressor outlet temperature $T_{2}$. Thus, Padilla's definition is not valid as the maximum heat defined by the denominator would result in values above the possible.

The more general expression for effectiveness from previous chapter will be used to define the global effectiveness in multi-heating cycles:

$$
\varepsilon=\frac{h_{5}-h_{6}}{q_{\max }}
$$

In this more general expression, the maximum heat $q_{\max }$ is defined as the exchanged heat in the heat exchanger when the pinch point (minimum temperature difference between low- and high-pressure sides) is zero.

Besides the global effectiveness, the effectiveness of the high temperature regenerator (HTR) is used to define the regeneration phase. In this case, the definition from Padilla et al. can be 
used because the maximum temperature of the additional heat is greater than the pinch point temperature, $T_{m}$. The definition of the HTR effectiveness is:

$$
\varepsilon_{H T R}=\frac{h_{5}-h_{9 \prime}}{h_{5}-h_{9 \prime} @ T_{8 \prime}}
$$

where $h_{9 \prime @ T_{8}}$ is the enthalpy at state 9' calculated based on the minimum temperature that the hot stream leaving the regenerator could reach, $T_{8}$.

The two mentioned effectiveness and the highest temperature of the additional heat will define the inside of the cycle. Considering the diagram from Figure 3.10 with the points 1245 already defined, point 6 will be calculated by means of the global effectiveness. This implies that a regenerative cycle with or without additional heats and the same global effectiveness will have the same point 6 , i.e., the precooler will extract the same heat. The heat temperature defines point 8' and the HTR effectiveness points 9' and 3'. The definition of point 9' determines point 7' due to the energy balance between lines 2-6 and 7'-9'. This means that the effectiveness of the low-temperature heat exchanger will be obtained as a result, always fulfilling the requirement of having a minimum temperature difference greater than, at least, $0{ }^{\circ} \mathrm{C}$.

A balance of energy in the regenerators helps to prove that a cycle with the same points 1245 and the same global effectiveness will need the same amount of total heat, either if there is additional heat or not. In a multi-heating cycle such as the one in Figure 3.10, the balance to the regenerators would be:

$$
\left(h_{9 \prime}-h_{6}\right)+\left(h_{5}-h_{9^{\prime}}\right)=\left(h_{7,}-h_{2}\right)+\left(h_{3 \prime}-h_{8^{\prime}}\right)
$$

The balance to the regenerators in a cycle without the additional heat would be:

$$
h_{5}-h_{6}=h_{3}-h_{2}
$$

The combination of Equations 3.9 and 3.10 involves the following result:

$$
h_{3 \prime}-h_{3}=h_{8},-h_{7 \prime}
$$

This means that the heat that the multi-heating cycle does not supply in the main source is supplied by the additional heat. Therefore, the sum of both primary heat, $q_{p h}$, and additional heat, $q_{a h}$, must be the total heat, $q_{\text {total }}$, which was already stated in Equation 3.3. This total heat would be also the heat supplied in a simple cycle without additional heats and the same global effectiveness. 
The regeneration process in multi-heating cycles with one additional heat are defined by two effectiveness (global and HTR) and the heat temperature. If more additional heats are supplied at different temperatures, a new effectiveness and a heat temperature will be needed to define each new additional heat. This new effectiveness will be the one corresponding to the intermediate heat exchanger after the new additional heat. In this way, the effectiveness of the first regenerator will always be a result.

The definition of thermal efficiency in Equation 2.73 states that cycles with the same compressor work, turbine work and total heat will have the same thermal efficiency. This means that two cycles, one without additional heat and the other with one additional heat defined by its maximum temperature and the HTR effectiveness, will have the same thermal efficiency. Multi-heating cycles and simple regenerative cycles with the same effectiveness may be compared with the only difference of the heat temperature.

The exergy measures the cost of the heat source (Müller et al., 2011), relating it with the heat source temperature. Therefore, an exergetic analysis will appreciate the benefits of multiheating cycles coming from the lower cost of heat sources at lower temperature.

The parameters from Table 3.2 will be used as base case to analyse multi-heating unless otherwise specified. All the parameters are dimensionless. Most of them will be varied in order to study its influence in the cycle performance.

Table 3.2. Parameters used to analyze multi-heating pericritical cycles

\begin{tabular}{ll}
\hline Parameter & Value \\
\hline Reduced high-side pressure, $P_{H, r}$ & 3 \\
Reduced low-side pressure, $P_{L, r}$ & 1 \\
Reduced compressor inlet temperature, $T_{c, \text { in, } r}$ & 0.9 \\
Carnot factor, $\tau=T_{t, i n} / T_{c, \text { in }}$ & 3 \\
Compressor efficiency, $\eta_{c}$ & 0.85 \\
Turbine efficiency, $\eta_{t}$ & 0.85 \\
IHX effectiveness, $\varepsilon$ & 0.95 \\
Pressure losses, $\chi$ & 0.05 \\
\hline
\end{tabular}


The only new parameter with respect to previous chapter is the pressure loss, $\chi$. It will be considered that all the pressure losses occur in the primary heat exchanger. Thus, the pressure loss is defined as:

$$
\chi=\frac{P_{H}-P_{4}}{P_{H}}
$$

The pressure loss implies that the turbine pressure ratio will be lower than the compressor pressure ratio. But the effect will be similar to the effect of the turbine efficiency, as it was explained in previous chapter.

\subsubsection{Exergy analysis}

The maximum work from a Carnot engine supplied with a heat, $q$, operating between a heat source and a heat sink at temperatures $T_{h o t}$ and $T_{\text {cold }}$, respectively, is expressed in Equation 3.13 (Kim, Kim and Favrat, 2012). This work will be the exergy input, $e_{i n}$, from the heat source to the cycle.

$$
e_{\text {in }}=q \cdot \eta_{C}=q\left(1-\frac{T_{\text {cold }}}{T_{\text {hot }}}\right)
$$

The exergy input of each heat source depends on the supplying temperature. Thus, the heats added in multi-heating cycles will have different exergy depending on the heat source temperature. In order to generalize the analysis and avoid the definition of the heat source, the heat source temperature will be the maximum temperature of the heat in the cycle. In the same way, the heat sink temperature will be minimum temperature of the cycle. Thus, the heats in Figure 3.10 would be defined by temperatures $T_{8}$, and $T_{4}$, and the heat sink by temperature $T_{1}$.

The total exergy input of a cycle with several additional heats will be the sum of the exergy inputs of each heat. The heat supplied at the maximum temperature (which is the turbine inlet temperature) will have higher specific costs (in terms of Carnot efficiency, $\eta_{c}$ ) than the rest of the heats. Therefore, reducing this heat and substituting it by additional heats at lower temperatures will reduce the total exergy input.

Figure 3.12 shows the importance of the additional-heat temperature, $T_{a h}$, in a multi-heating cycle. Continuous lines represent the heats, $q$, and dashed lines the Carnot efficiencies, $\eta_{c}$, at each temperature, $T_{a h}$. The higher the additional-heat temperature, $T_{a h}$, the larger this additional heat, $q_{a h}$, is. This involves a decrease of the primary heat, $q_{p h}$, as the total heat, $q_{\text {total }}$, is constant. Reducing the primary heat will be beneficial exergetically speaking because 
it will reduce the exergy input. However, this benefit is counteracted by the increase of the additional heat and its Carnot efficiency, which will increase its exergy input.

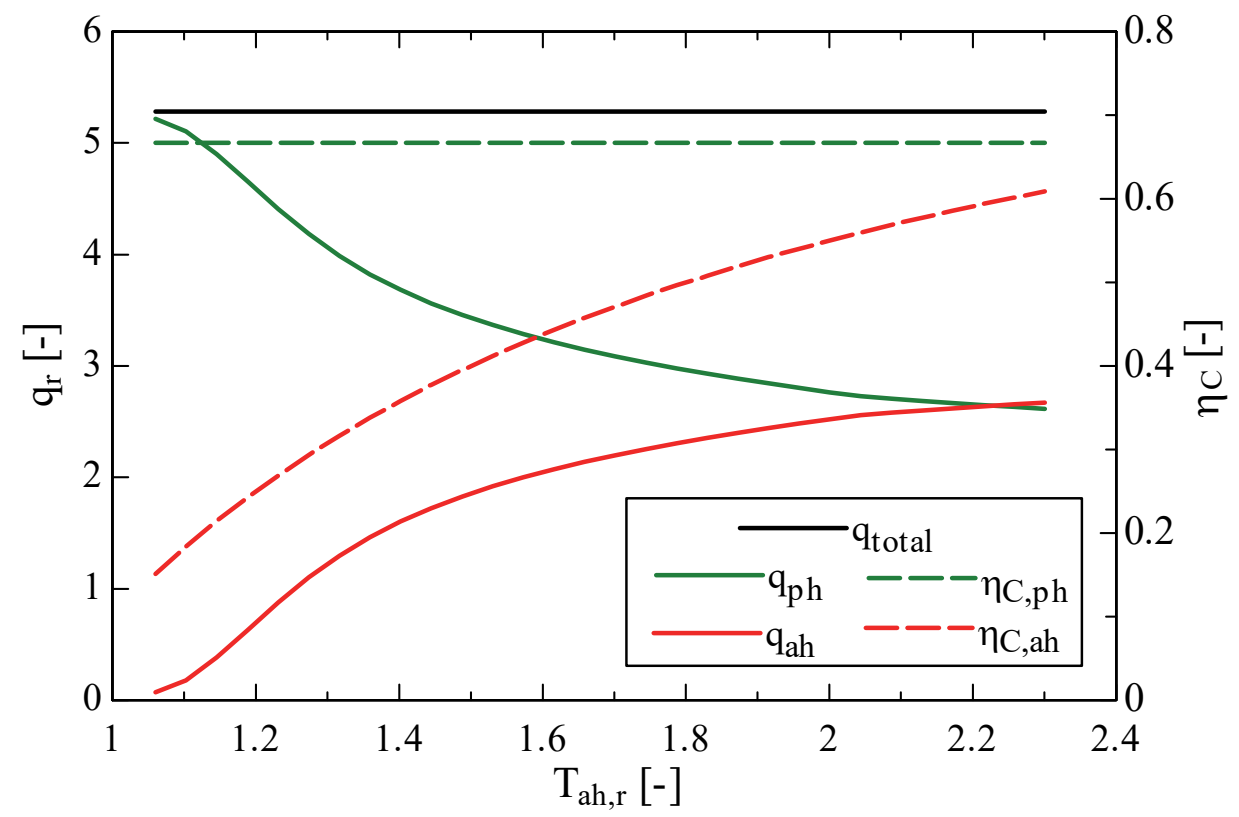

Figure 3.12. Heats, $q$, of a multi-heating cycle and its associated Carnot efficiencies, $\eta_{c}$, as a function of the additional-heat temperature, $T_{a h}$. Primary heat, $p h$, additional heat, $a h$, and total heat, total, are represented.

The total exergy input is the sum of the exergies of primary and additional heats. In the case of a multi-heating cycle with one additional heat, this total exergy input is expressed in Equation 3.14 .

$$
e_{\text {in,total }}=\left(q_{\text {total }}-q_{a h}\right) \eta_{C, p h}+q_{a h} \eta_{C, a h}
$$

Figure 3.13 shows the exergy inputs of the primary heat, $e_{i n, p h}$, additional heat, $e_{i n, a h}$, and total heat, $e_{i n, t o t a l}$, as a function of the additional-heat temperature, $T_{a h}$. The exergy input of the additional heat increases with the temperature and the primary heat exergy input decreases. The decrease of the primary-heat exergy is produced by the increase of the additional heat. It is seen in Figure 3.12 that most of the additional heat can be supplied at temperatures under $T_{r}=1.5$. This means that increasing the additional-heat temperature up to this temperature strongly reduces the primary heat, and so its exergy input. Raising this temperature also involves an increase in the exergy input of the additional heat, but the low Carnot efficiencies reduce its impact. The sum of primary-heat exergy and additional-heat exergy (as shown in Equation 3.14) results in a total exergy input with a minimum in its curve at a temperature $T_{a h, r}=1.45$. 


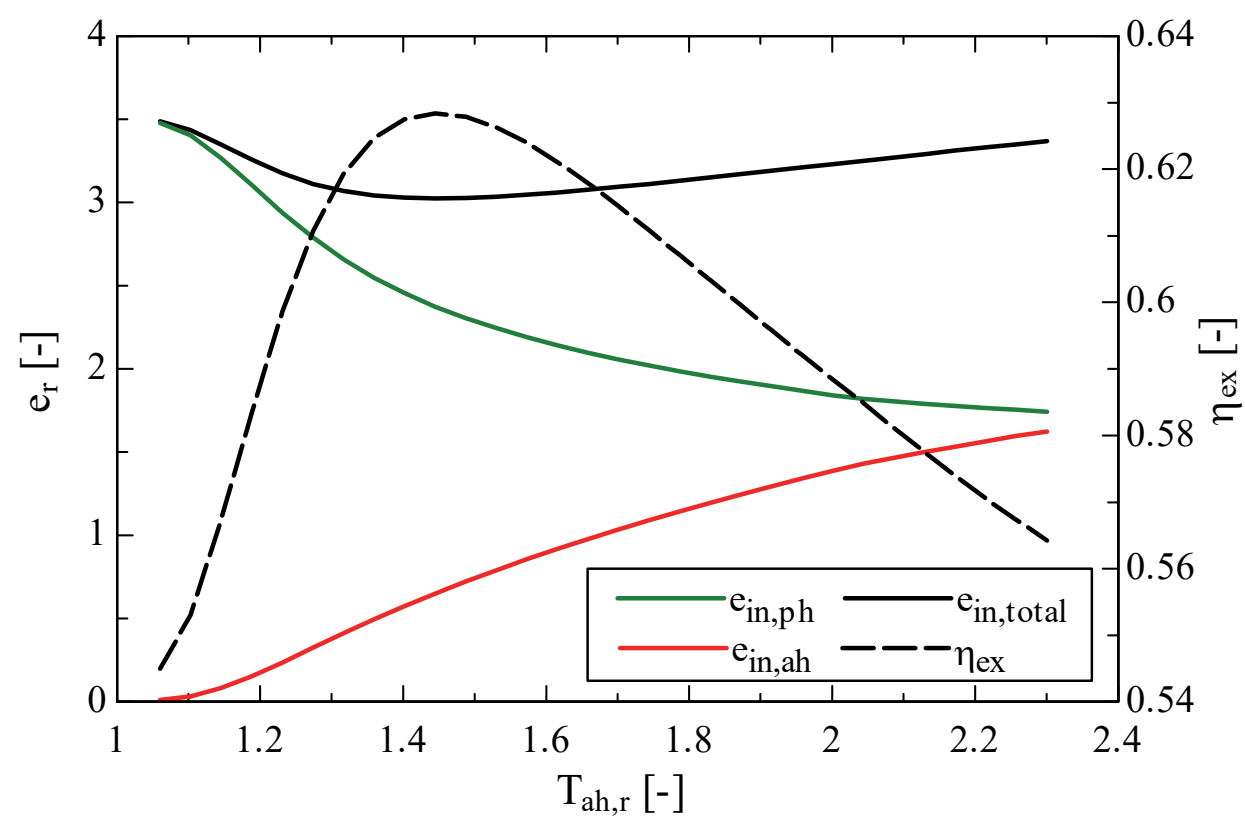

Figure 3.13. Exergy inputs of the primary heat, $e_{i n, p h}$, additional heat, $e_{i n, a h}$, and total heat, $e_{\text {in,total, }}$ and cycle exergetic efficiency, $\eta_{e x}$, as a function of the additional-heat temperature, $T_{a h .}$

A small exergy input means a low cost of thermal energy required by the cycle. But an optimization of a thermodynamic cycle, besides reducing the thermal energy cost, seeks to increase the generated work. The relation between the generated work and the cost of the energy input may be analysed by means of the second law efficiency:

$$
\eta_{e x}=\frac{w_{t}-w_{c}}{e_{\text {in,total }}}=\frac{w_{t}-w_{c}}{\sum e_{i n, j}}
$$

where $e_{i n, j}$ is the exergy input of each heat supplied to the cycle at a different temperature, including the main heat source.

The multi-heating cycle studied in Figure 3.13 has constant compressor and turbine works. Therefore, the minimum exergy input defines the maximum exergetic efficiency. The point with the minimum exergetic efficiency to the left of Figure 3.13 corresponds to a simple regenerative cycle. The other end (to the right) of the exergetic efficiency curve represents a cycle with the additional heat supplied at the minimum temperature of the primary heat, i.e., the cycle is basically a simple regenerative cycle, but instead of supplying the total heat at the turbine inlet temperature, the heat is supplied at two different temperatures. The result is that the exergy efficiency at this point is slightly greater than the minimum found in the other end of the curve. 


\subsubsection{Exergy losses}

The exergy efficiency can also be expressed as a function of the exergy losses of each component, $i_{j}$ :

$$
\eta_{e x}=1-\sum \frac{i_{j}}{e_{\text {in,total }}}
$$

The exergy loss is defined as the input exergy minus the outlet exergy, and the specific exergy of a point, $e_{j}$, is expressed as:

$$
e_{j}=h_{j}-h_{a m b}-T_{a m b}\left(s_{j}-s_{a m b}\right)
$$

where the subscript $a m b$ stands for ambient conditions, which are assumed to be the heat sink conditions.

The following equations express the exergy loss or irreversibility of the components of a multi-heating cycle with one additional heat such as the one shown in Figure 3.10:

$$
\begin{gathered}
i_{c}=w_{c}-\left(e_{2}-e_{1}\right) \\
i_{t}=\left(e_{4}-e_{5}\right)-w_{t} \\
i_{L T R}=\left(e_{9 \prime}-e_{6}\right)-\left(e_{7 \prime}-e_{2}\right) \\
i_{H T R}=\left(e_{5}-e_{9 \prime}\right)-\left(e_{3 \prime}-e_{8 \prime}\right) \\
i_{p h}=e_{i n, p h}-\left(e_{4}-e_{3 \prime}\right) \\
i_{a h}=e_{i n, a h}-\left(e_{8 \prime}-e_{7 \prime}\right) \\
i_{p c}=e_{6}-e_{1}
\end{gathered}
$$

Figure 3.14 shows the irreversibilities of a multi-heating cycle with one additional heat at the conditions of Table 3.2 as a function of the additional-heat temperature, $T_{a h}$. Precooler, compressor and turbine values are constant as they do not change with the additional-heat temperature. The exergy losses of the heats and the intermediate heat exchangers depend on the additional-heat temperature, resulting in a total exergy loss curve with a minimum at temperature $T_{a h, r}=1.45$. LTR and HTR irreversibilities, $i_{L T R}$ and $i_{H T R}$, experience the largest variations, which implies a greater impact in the exergy efficiency than the rest of the cycle components. 


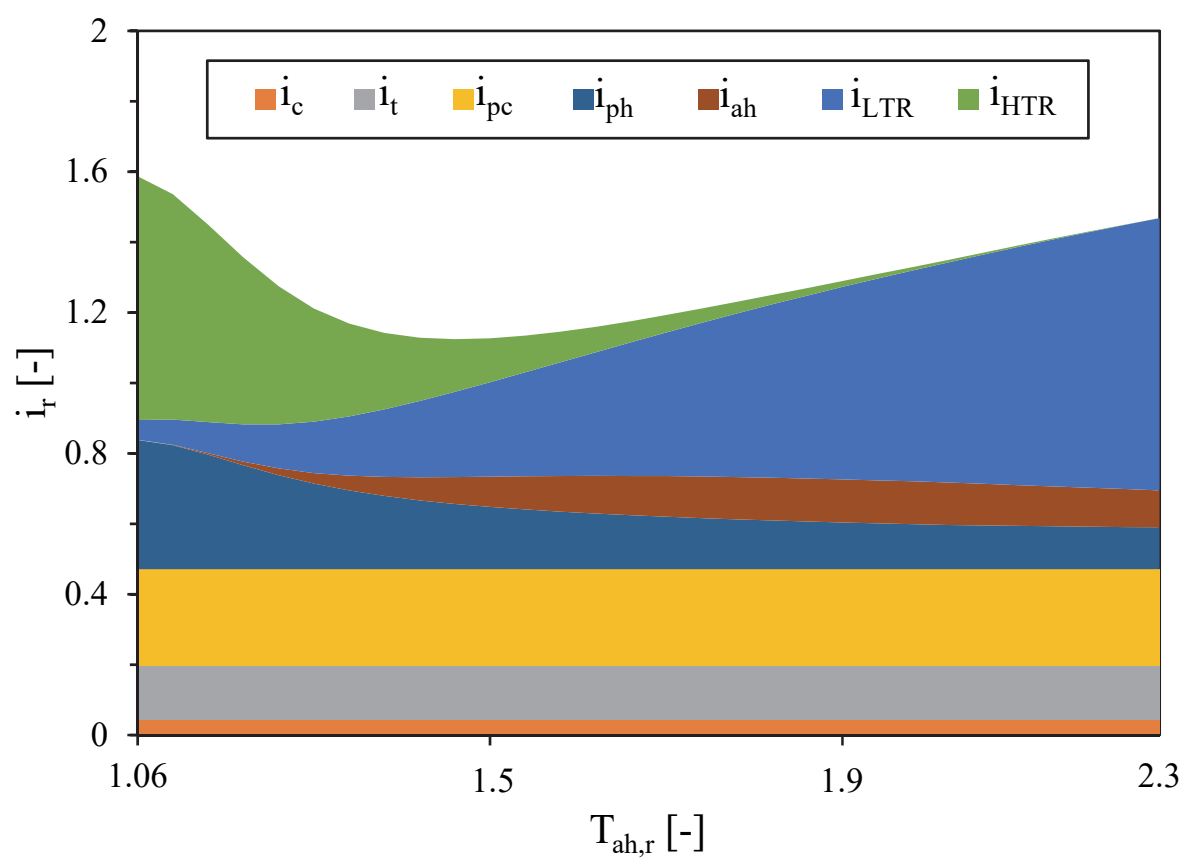

Figure 3.14. Exergy losses, $i$, of the components of a multi-heating cycle as a function of the additional-heat temperature, $T_{a h}$. These components are: compressor $(c)$, turbine $(t)$, precooler $(p c)$, primary heat exchanger $(p h)$, additional heat exchanger $(a h)$, low temperature regenerator $(L T R)$, high temperature regenerator $(H T R)$.

The irreversibility of the additional heat, $i_{a h}$, to the left of Figure 3.14 is zero because there is no additional heat added yet. This irreversibility increases with the additional-heat temperature, $T_{a h}$, while the primary heat irreversibility, $i_{p h}$, decreases. The irreversibilities of the heats would be larger if greater heat source temperatures were considered instead of the highest temperatures of the heats in the cycle. This means that the total irreversibility curve could change. The heat sink irreversibility would be also higher if a lower heat sink temperature were used to calculate the exergy loss. However, the heat sink irreversibility would still be constant and therefore would not affect the behavior of the total irreversibility curve.

The LTR irreversibility, $i_{L T R}$, at $T_{a h, r}=1.06$, i.e., when there is not additional heat added, is due to the regeneration before the pinch point. Although it is difficult to appreciate, the LTR irreversibility increases almost linearly with the temperature, while the HTR irreversibility, $i_{H T R}$, decreases exponentially. The result is that the point of minimum exergy loss is achieved by reducing the total irreversibility of the heat exchangers. Note that the irreversibility in a heat exchanger is given by the variation of entropy. Reducing the entropy in the regeneration of multi-heating cycles increases the exergetic efficiency. 


\subsubsection{Additional heats}

Multi-heating cycles with one additional heat seem to show important improvements with respect to simple regenerative cycles. Thus, it is interesting to analyse the benefits of supplying more additional heats. Figure 3.15 shows the exergy efficiency, $\eta_{e x}$, of a cycle with two additional heats as a function of the temperature of the first additional heat, $T_{a h 1}$. The temperature of the second additional heat, $T_{a h 2}$, is calculated to maximize the exergy efficiency. The exergy efficiency in a multi-heating cycle with two additional heats at $T_{a h 1}=1.29$ and $T_{a h 2}=1.67$ is $\eta_{e x}=0.66$, which is higher than the efficiency obtained with one additional heat, $\eta_{e x}=0.63$.

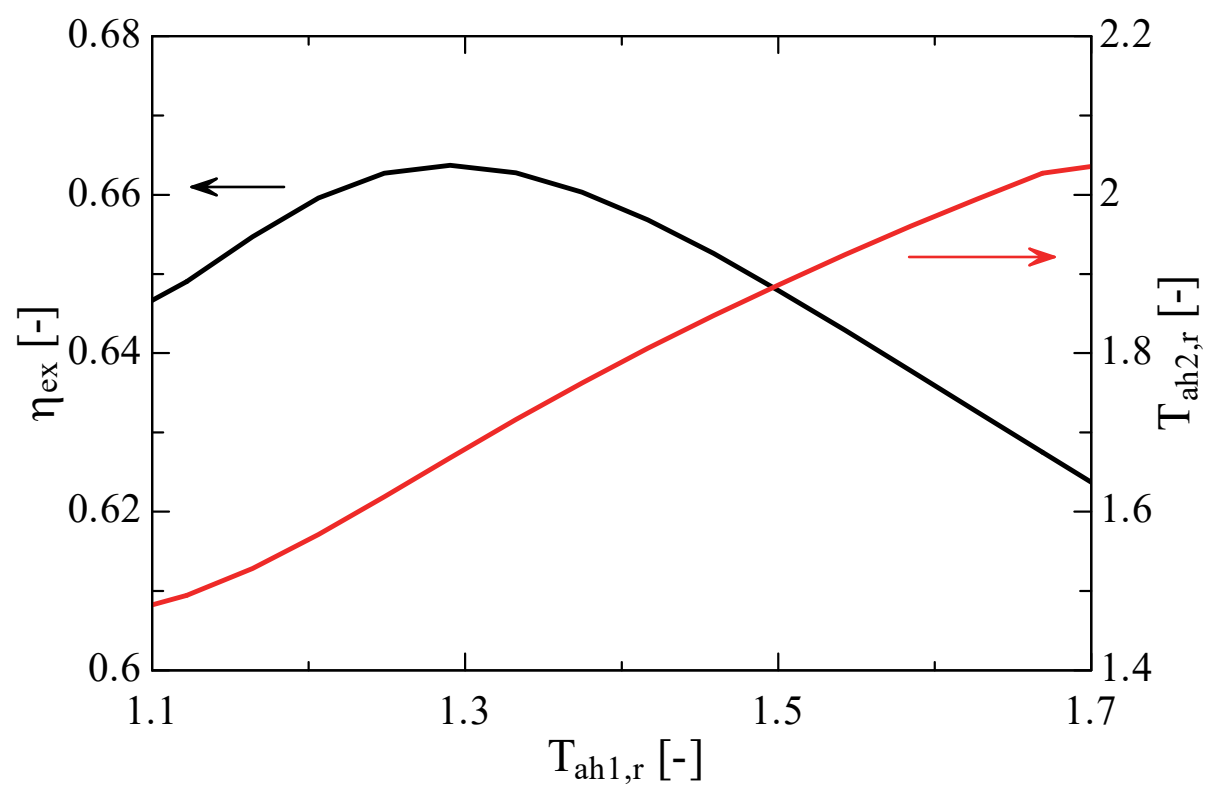

Figure 3.15. Exergy efficiency, $\eta_{e x}$, and temperature of the second additional heat, $T_{a h 2}$, of a multi-heating cycle with two additional heats as a function of the temperature of the first additional heat $T_{a h l}$.

In the same way, more additional heats can be supplied to the cycle. Figure 3.16 shows the exergy efficiency, $\eta_{e x}$, of the cycle with up to six additional heats. The temperatures of these heats are optimized to maximize the exergy efficiency. Supplying additional heats to the cycle increases the exergetic efficiency, but this increment decreases as the number of heats grows. The relative increase of exergy efficiency is defined in Equation 3.25 in order to quantify the benefit of additional heats.

$$
\Delta \eta_{e x}=\frac{\eta_{e x, N_{a h}}-\eta_{e x, N_{a h}-1}}{\eta_{e x, N_{a h}-1}}
$$




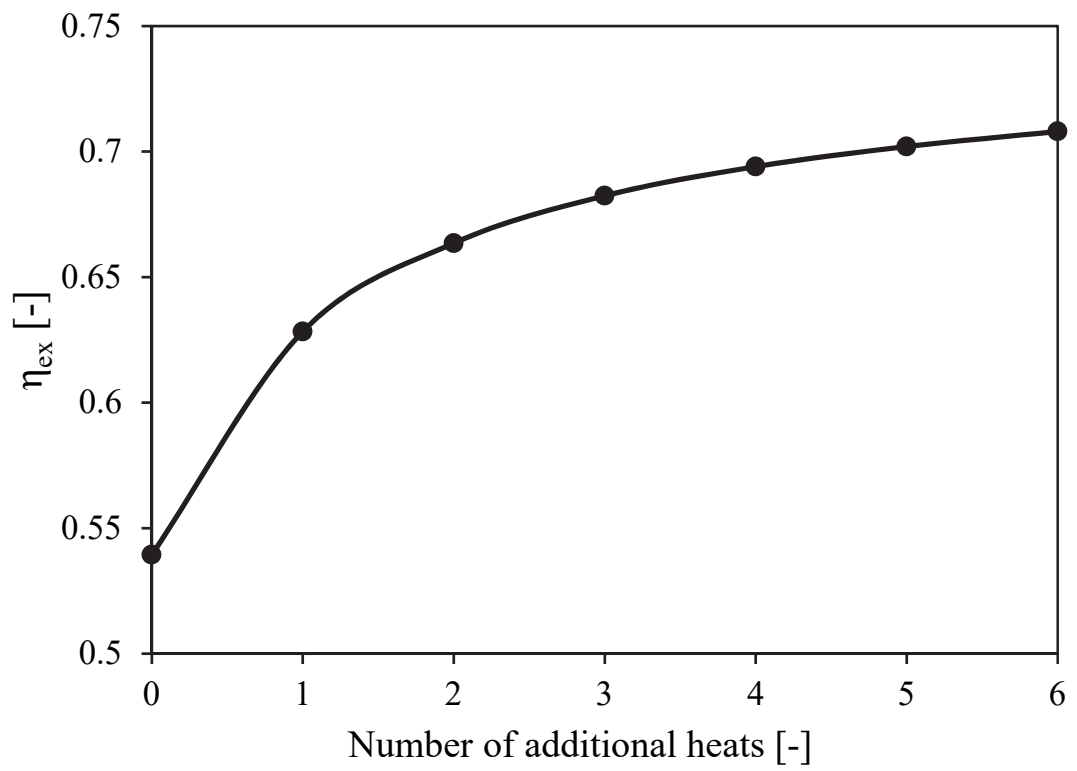

Figure 3.16. Exergetic efficiency, $\eta_{e x}$, of a multi-heating cycle as a function of the number of additional heats

The relative increase of adding one additional heat $\left(N_{a h}=1\right)$ with respect to the simple case $\left(N_{a h}=0\right)$ is $16 \%$. The relative increase of adding another heat is $6 \%$ and if a third heat is added then this increase is only 3\%. A greater number of additional heats involves a more complex cycle, and therefore more expensive. A balance between complexity and efficiency increase will define the optimum cycle layout.

\subsection{Best conditions for multi-heating cycles}

Last section has shown the optimization of multi-heating cycles with the parameters from Table 3.2. This section will analyse how these parameters affect the benefit of multi-heating cycles. The impact of the compression inlet temperature, the limiting pressures and the components inefficiencies will be studied. The purpose is to find the conditions in which multi-heating cycles suppose a greater improvement with respect to simple regenerative cycles.

\subsubsection{The discontinuity line}

Chapter 2 showed that pericritical cycles in the liquid region have larger heat losses during the regeneration due to the mismatch of specific heats between the low and high-pressure sides. The additional heats in multi-heating cycles compensate these regeneration heat losses. Thus, the larger the heat loss, the bigger the potential of multi-heating cycles is. Pericritical 
cycles with the compressor working in the liquid region (i.e., those crossing the discontinuity line during the regeneration) will take more advantage of multi-heating to increase the exergy efficiency. Moreover, the compressor outlet temperatures of these cycles are lower than in cycles with the compressor working in the gas-like region. This implies that the additional heats can be supplied at lower temperatures, which benefits the exergy efficiency.

Figure 3.17 represents the exergy efficiency, $\eta_{e x}$, of multi-heating cycles with different number of additional heats, $N_{a h}$, as a function of the low-pressure side, $P_{L, r}$. The influence of the terms $w_{c} / w_{t}$ and $\Delta h_{r g} / w_{t}$ in the efficiency is shown in Figure 3.18. The compressor inlet temperature in the cycles is $T_{c, i n, r}=0.95$ and $T_{c, i n, r}=1.05$. The high-side pressure $P_{H, r}=3$ is constant in all the cases. The additional heat supplies have been optimized in all the cycles to obtain the maximum exergy efficiency.
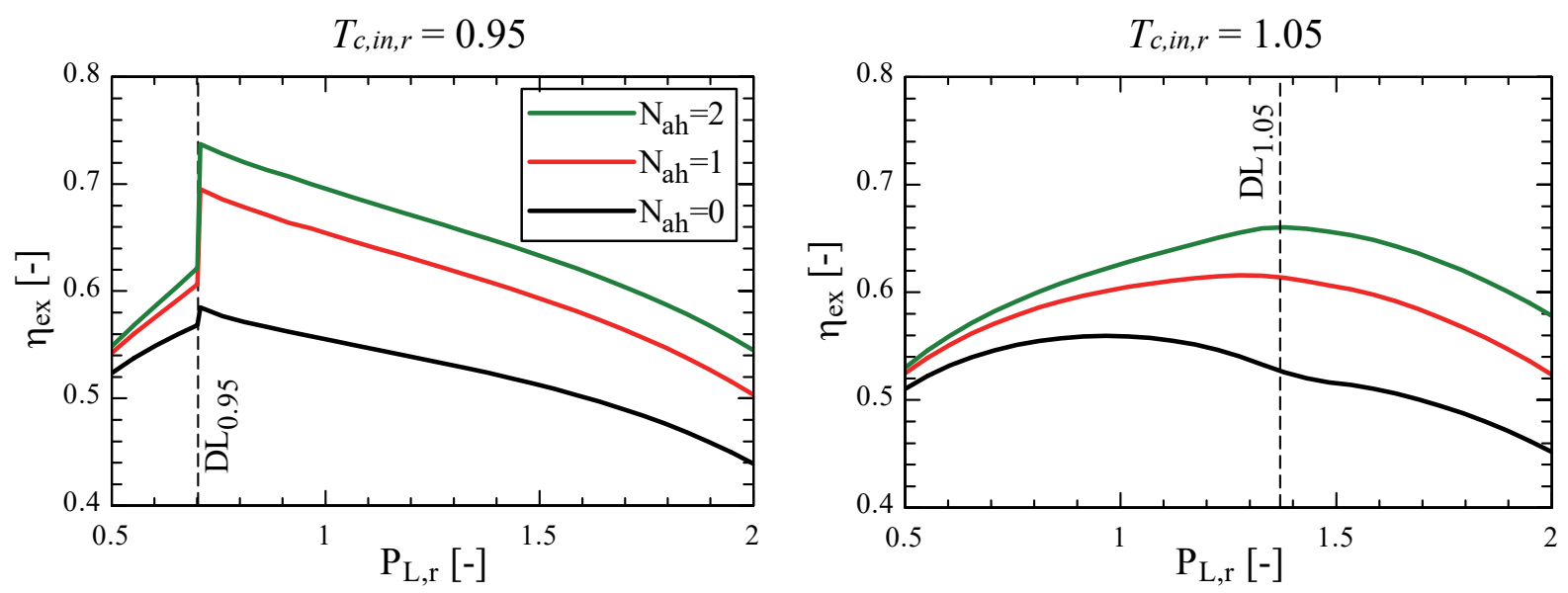

Figure 3.17. Exergetic efficiency, $\eta_{e x}$, of a multi-heating cycle with different numbers of additional heats, $N_{a h}$, as a function of the low-pressure side, $P_{L, r}$. The high pressure is set to $P_{H, r}=3$.
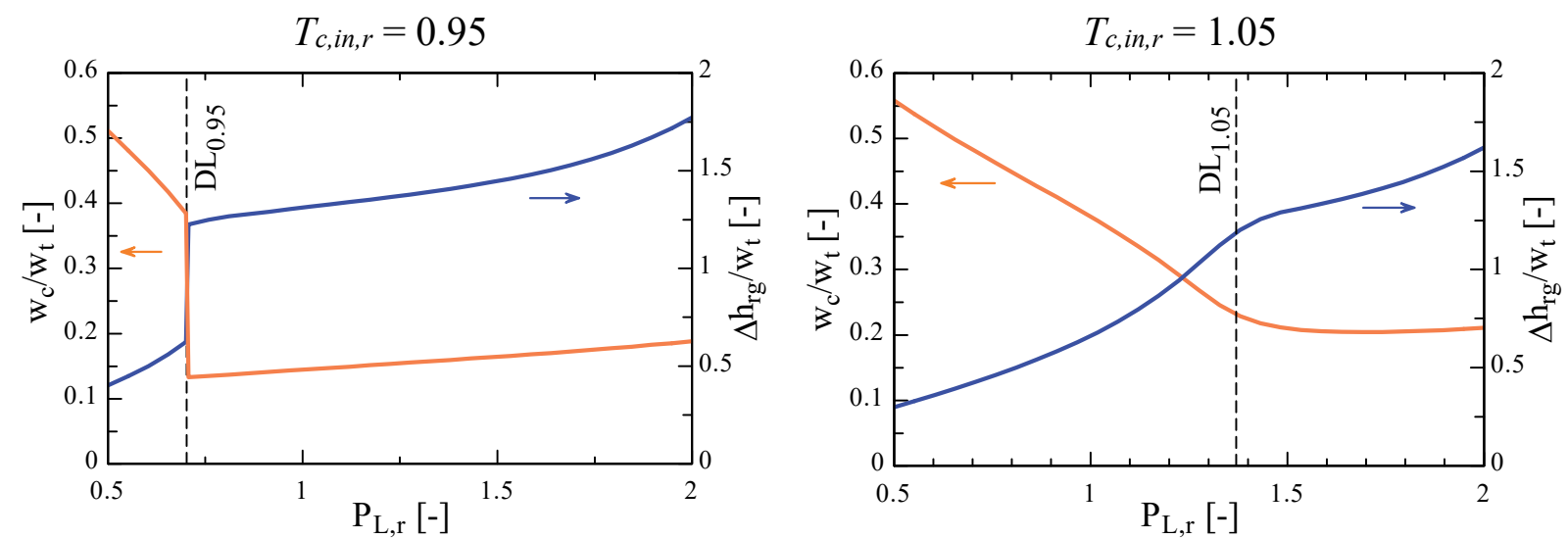

Figure 3.18. Parameters $w_{c} / w_{t}$ and $\Delta h_{r g} / w_{t}$ of a pericritical cycle as a function of the lowpressure side, $P_{L, r}$. The high pressure is set to $P_{H, r}=3$. 
Note that the curves of the simple cycles $\left(N_{a h}=0\right)$ will have the same shape than the thermal efficiency because the exergy efficiency in these cases is the thermal efficiency divided by the Carnot efficiency.

When the compressor inlet temperature is $T_{c, i n, r}=0.95$, the maximum exergy efficiency is obtained in the liquid region (low-side pressure above the discontinuity line) either if there are or there are not additional heats. The parameter $w_{c} / w_{t}$ rules the exergy efficiency, i.e., the maximum efficiency is obtained at the minimum $w_{c} / w_{t}$ value. Nevertheless, supplying additional heats makes the parameter $w_{c} / w_{t}$ to rule even more the maximum exergetic efficiency. Positioning the compressor in the liquid region has a greater benefit in multiheating cycles $\left(N_{a h}>0\right)$. Multi-heating cycles reduce the influence of the parameter $\Delta h_{r g} / w_{t}$ in the exergy efficiency because the regeneration heat loss is managed by the additional heats.

The optimum low-side pressure when the compressor inlet temperature is $T_{c, i n, r}=1.05$ depends on the number of additional heats. When the cycle is simple $\left(N_{a h}=0\right)$, reducing $\Delta h_{r g} / w_{t}$ at the expense of raising $w_{c} / w_{t}$ increases the exergetic efficiency till pressures close to the critical one, where the maximum exergy efficiency is found. When additional heats are supplied, the maximum exergy efficiency is obtained closer to the point of minimum $w_{c} / w_{t}$. It is not obtained at this point because the enthalpy loss $\Delta h_{r g} / w_{t}$ still has a big impact in the exergy efficiency. This regeneration enthalpy loss is managed by the additional heats, but not eliminated. The optimum low-side pressure is located in a region where the compression work is small and where the enthalpy loss is not very high: the discontinuity band.

The influence of the discontinuity line is greater in multi-heating cycles $\left(N_{a h}>0\right)$ than in simple cycles $\left(N_{a h}=0\right)$. Even at conditions where simple cycles have the optimum low-side pressure far from the discontinuity line, the supply of additional heats approaches it. The importance of the parameter $w_{c} / w_{t}$ increases because the regeneration heat loss $\Delta h_{r g} / w_{t}$ is compensated with the additional heats. Thus, multi-heating cycles with lower compressor inlet temperatures increase more the exergy efficiency. On the other hand, multi-heating cycles with higher compressor inlet temperatures increase less the exergy efficiency, but they reduce the optimum pressure ratio.

\subsubsection{Pressure limits}

The optimum pressure ratio depends on the compressor inlet temperature and the number of additional heats. Nevertheless, it will also depend on other factors such as the high-side pressure. The influence of the number of additional heats in the optimum low-side pressure is 
shown in Figure 3.19 for different values of high-side pressure, $P_{H}$, in the cases of a compressor inlet temperature $T_{c, i n, r}=0.95$ and $T_{c, i n, r}=1.05$. The optimum pressures can be divided into two groups: low-side pressures close to the discontinuity line and low-side pressures far away from it. These two groups are perfectly marked in the case of $T_{c, i n, r}=0.95$ and more difficult to appreciate due to the softer variations in the case of $T_{c, i n, r}=1.05$.
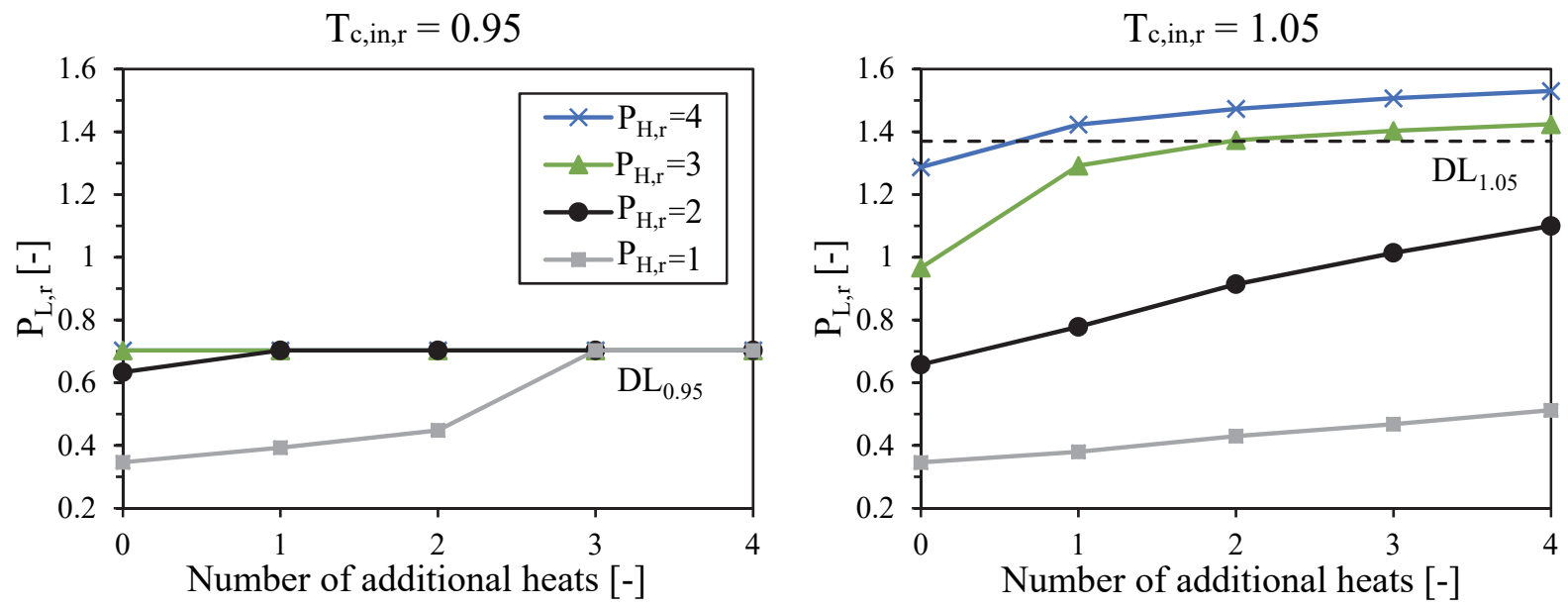

Figure 3.19. Optimum low-side pressure, $P_{L, r}$, as a function of the number of additional heats for different high-side pressures, $P_{H, r}$.

The low-side pressures far away from the discontinuity line belong to cycles with high-side pressures close or below to the discontinuity line. The small range of pressures between these high-side pressures and the discontinuity line implies that the small compression work cannot compensate the large regeneration heat loss. Thus, the optimum low-side pressure is at pressures below the discontinuity line where the enthalpy loss in the regeneration is low, i.e., in the gaseous region.

Supplying additional heats reduce the effect of the regeneration heat loss, which involves higher optimum low-side pressures. In some of these cases one extra additional heat makes the low-side pressure to jump into the discontinuity line (or close to it in the case of $\left.T_{c, i n, r}=1.05\right)$. The characteristics needed by a multi-heating cycle to have the optimum lowside pressure in the discontinuity line are either to have a high high-side pressure (enough distance between this pressure and the discontinuity line) or a large number of additional heats.

The effect of the high- and low-side pressures in the exergy efficiency, $\eta_{e x}$, is shown in Figure 3.20. The efficiency in all the cases increases with the number of additional heats. The cycles with low-side pressure close to the discontinuity achieve higher efficiency increments with the additional-heat supplies. The biggest increment in these cycles occurs with the supply of 
the first additional heat. Supplying more additional heats slows down the relative increase. After four additional heats, the exergetic efficiency of these cycles with the low-side pressure close to the discontinuity tends to a similar threshold in which most of the regeneration heat loss is managed by the multi-heating.
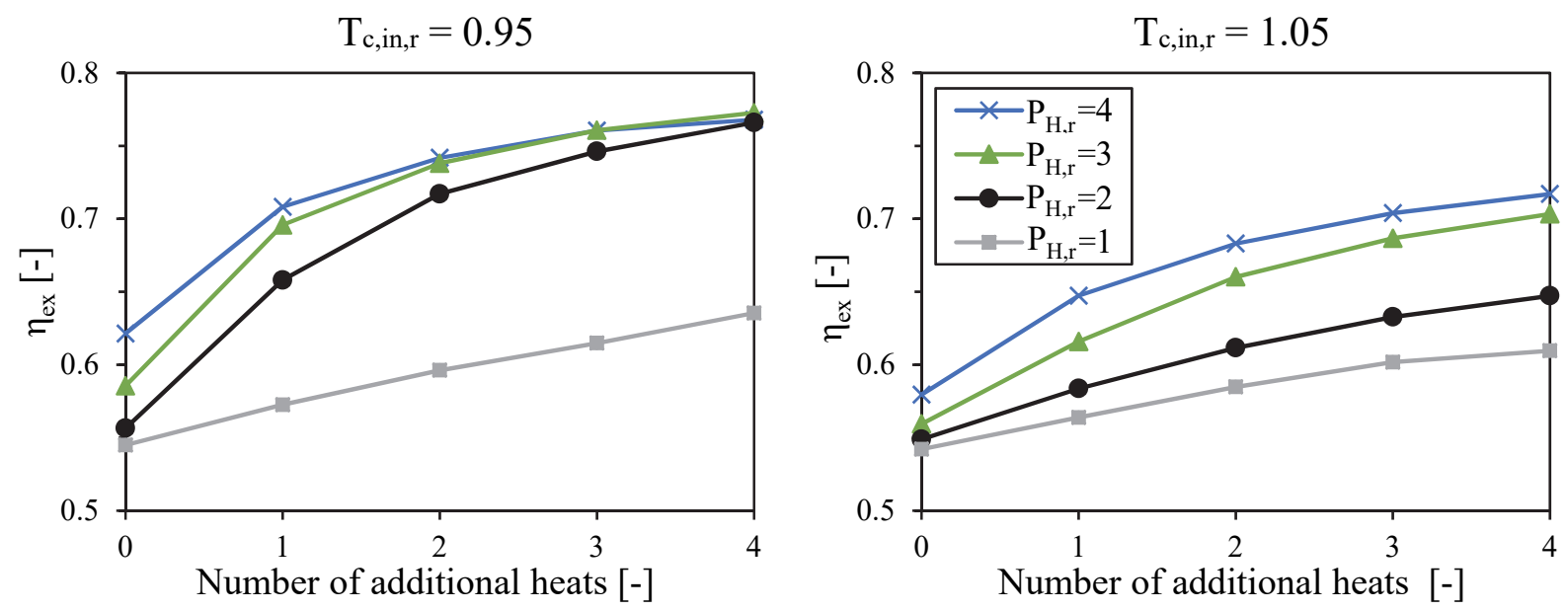

Figure 3.20. Exergy efficiency, $\eta_{e x}$, as a function of the number of additional heats for different high-side pressures, $P_{H, r}$

The increase of efficiency when raising the high-side pressure depends on the number of additional heats and the compressor inlet temperature. For example, the biggest increase of efficiency when there are no additional heats $\left(N_{a h}=0\right)$ is from $P_{H, r}=3$ to $P_{H, r}=4$ regardless of the compressor inlet temperature. However, when the number of additional heats is $N_{a h}=4$, the biggest increase is from $P_{H, r}=1$ to $P_{H, r}=2$ in the case of compressor inlet temperature $T_{c, i n, r}=0.95$ and from $P_{H, r}=2$ to $P_{H, r}=3$ in the case of compressor inlet temperature $T_{c, i n, r}=1.05$. Multi-heating cycles can achieve higher exergy efficiencies at high pressures and low compressor inlet temperatures.

Raising the high-side pressure in multi-heating cycles increases the exergetic efficiency in all the cases shown in Figure 3.20, except in the case with compressor inlet temperature $T_{c, i n, r}=0.95$ and $N_{a h}=4$ additional heats. The threshold of the cycle with $P_{H, r}=4$ is reached before the threshold of the cycle with $P_{H, r}=3$, and the efficiency of the cycle with $P_{H, r}=3$ continues to grow surpassing the efficiency of the cycle with $P_{H, r}=4$. This means that raising the high-side pressure in multi-heating cycles with many additional heats may not always be profitable. Anyway, raising the high-pressure side and the number of additional heats involve higher costs, therefore these variations must convey a worthwhile increase in efficiency to be considered a candidate solution. 


\subsubsection{Temperature limits}

Previous results of pericritical cycles with multi-heating show the cases with compressor inlet temperatures $T_{c, i n, r}=0.95$ and $T_{c, i n, r}=1.05$. Figure 3.21 shows the influence of the compressor inlet temperature, $T_{c, i n, r}$, in multi-heating cycles with $N_{a h}$ additional heats and a high-side pressure $P_{H, r}=3$. The exergetic efficiency, $\eta_{e x}$, and the low pressure side, $P_{L, r}$, needed to obtain these efficiencies are represented in the figure. The results show the tendency seen in $T_{c, i n, r}=0.95$ and $T_{c, i n, r}=1.05:$ the lower the compressor inlet temperature, the greater the increase of efficiency when supplying additional heats. Moreover, the optimum low-side pressure is ruled by the discontinuity line, especially at low compressor inlet temperatures and when additional heats are supplied to the cycle.
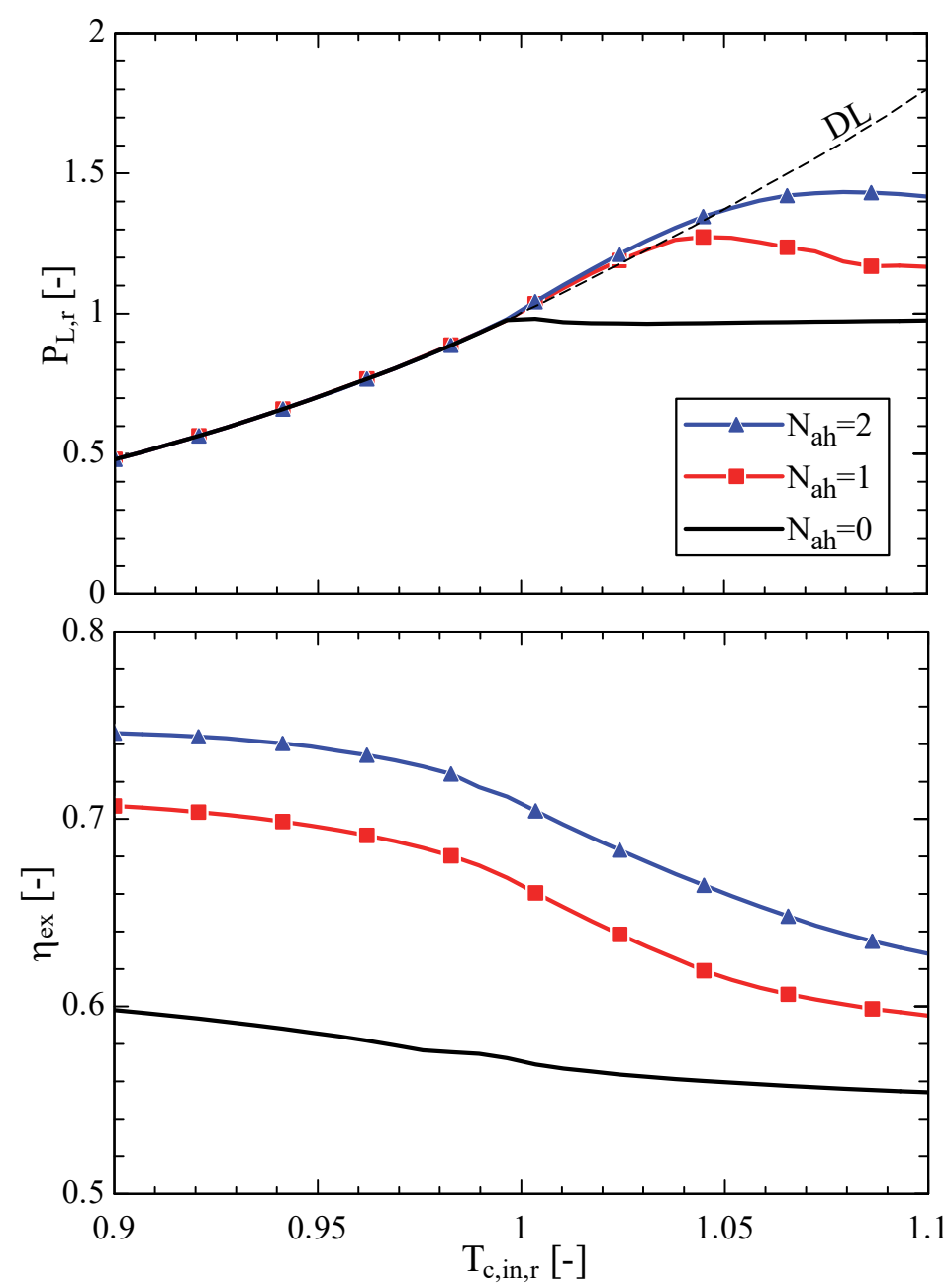

Figure 3.21. Exergy efficiency, $\eta_{e x}$, and optimum low-side pressure, $P_{L, r}$, of a multi-heating cycle with $N_{a h}$ additional heats as a function of the compressor inlet temperature, $T_{c, i n, r}$.

In the range of temperatures between $T_{c, i n, r}=0.98$ and $T_{c, i n, r}=1.1$, the low-side pressure is below the discontinuity line when $N_{a h}=0$ (this also happens between $T_{c, i n, r}=0.98$ and 
$T_{c, i n, r}=1$, although it is difficult to appreciate). In these cases, the compressor works in the gaseous region in order to decrease the regeneration heat loss $\Delta h_{r g} / w_{t}$. However, when an additional heat is supplied $\left(N_{a h}>0\right)$, the regeneration heat loss is partially compensated, allowing higher low-side pressures to increase the exergetic efficiency. If enough additional heats are supplied to compensate the regeneration heat loss, the optimum low-side pressure will be the corresponding with the discontinuity line. The greater the number of additional heats, the greater the similarity between the discontinuity line and the optimum low-side pressure.

The regeneration heat loss $\Delta h_{r g} / w t$ is very similar in cycles with the low-side pressure side just above the discontinuity line (see Figure 3.18). Thus, the enthalpy loss $\Delta h_{r g} / w_{t}$ remains more or less constant in the cycles with compressor inlet temperature under $T_{c, i n, r}=0.98$ regardless of the number of additional heats. This means that all these cycles have a similar potential to take advantage of multi-heating. The result is that the increase of exergy efficiency between cycles with $N_{a h}=0$ and $N_{a h}>0$ is independent of the compressor inlet temperature for $T_{c, i n, r}<0.98$.

At temperatures above $T_{c, i n, r}=0.98$, the low-side pressure of cycles with $N_{a h}=0$ and $N_{a h}>0$ is different, and so it is its regeneration heat loss $\Delta h_{r g} / w t$. The lower the compressor inlet temperature, the lower the compressor outlet temperature and the larger the regeneration heat loss are. Therefore, larger additional heats may be supplied to multi-heating cycles with lower compressor inlet temperatures. The result is that, in the range of temperatures between $T_{c, i n, r}=0.98$ and $T_{c, i n, r}=1.1$, the lower the compression inlet temperatures, the greater the efficiency increase of supplying an additional heat.

It should be noted that the bigger pressure ratios characteristic from lower compressor inlet temperatures involve smaller heat exchanges during the regeneration. This means that the intermediate heat exchangers of these cycles will have less importance in the exergy efficiency, and the relevant factor will be the additional-heat temperature. In the limit, where there is no heat regeneration at all, the benefit of a multi-heating cycle would be the division of the primary heat into two, one at the primary-heat temperature, and the other one at the additional-heat temperature.

The influence of compressor inlet temperature and the high-side pressure has been studied separately to understand its behaviour. However, the results depend on both temperature and pressure at the same time. Figure 3.22 shows the exergy efficiency as a function of the highpressure side, $P_{H, r}$, and compressor inlet temperature, $T_{c, i n, r}$. for a simple cycle $\left(N_{a h}=0\right)$ and a 
multi-heating cycle with one additional heat $\left(N_{a h}=1\right)$. There are two clear trends valid in both cases: the efficiency increases by raising the high-side pressure and by lowering the compressor inlet temperature. However, the values are different: the efficiencies of the multiheating cycle are greater than those of the simple cycle.
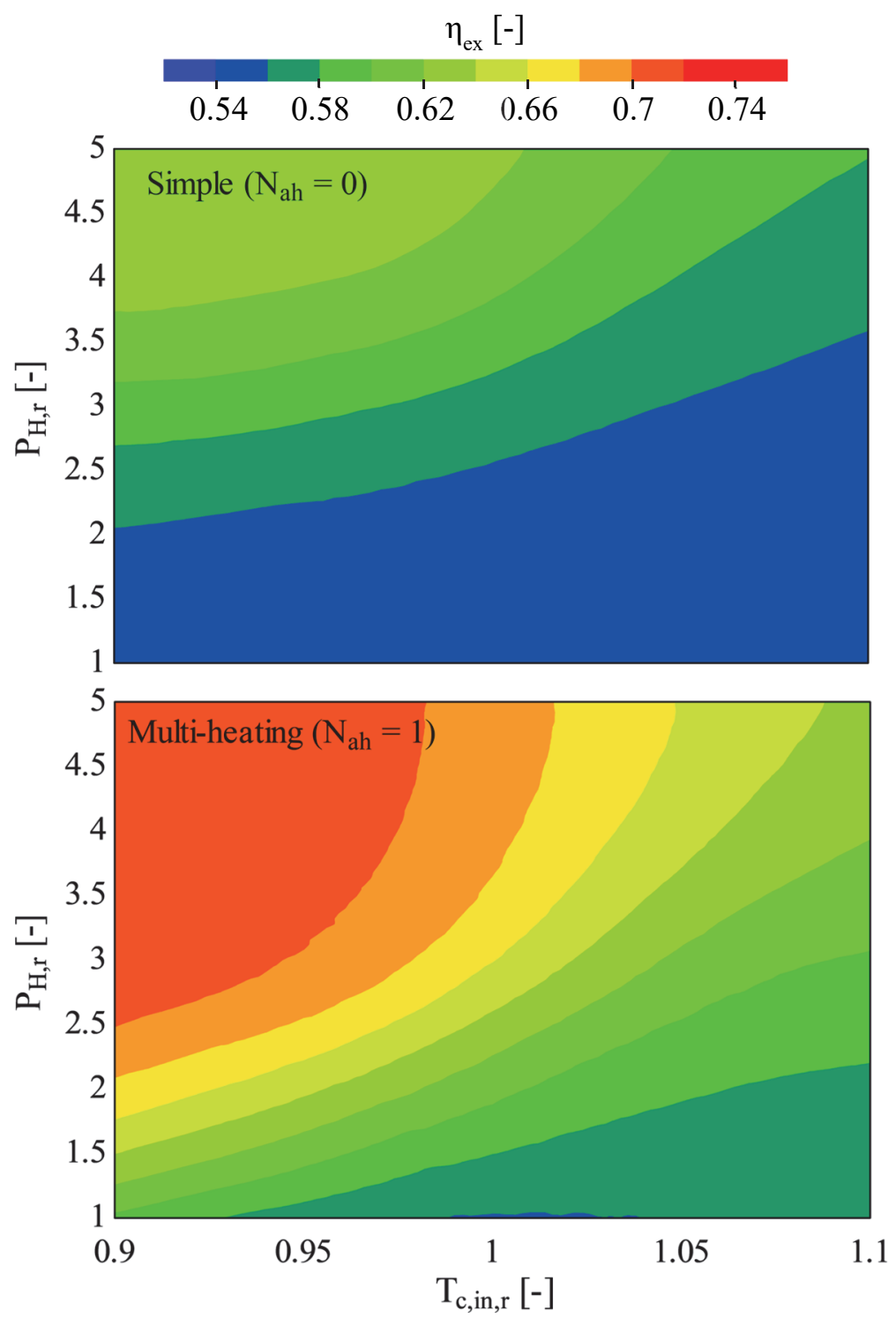

Figure 3.22. Exergy efficiency, $\eta_{e x}$, as a function of high-side pressure, $P_{H, r}$, and compressor inlet temperature, $T_{c, i n, r}$, in a simple cycle (top) and a multi-heating cycle (bottom)

It can be appreciated from the inclination of the isoexergetic lines that reducing the compressor inlet temperature has a greater benefit in multi-heating cycles. Moreover, the greater proximity of the isoexergetic lines in these cycles indicates that raising the high-side pressure also has a greater benefit.

Obtaining the maximum exergy efficiency by raising the high-side pressure and by reducing the compressor inlet temperature does not mean that the biggest potential of multi-heating 
cycles is obtained in the same way. The potential of multi-heating cycles can be analysed by calculating the relative increase of exergy efficiency of supplying an additional heat to a simple cycle with no additional heats. Figure 3.23 shows this relative increase $\Delta \eta_{e x}$ as a function of high-pressure side, $P_{H, r}$, and compressor inlet temperature, $T_{c, i n, r}$.

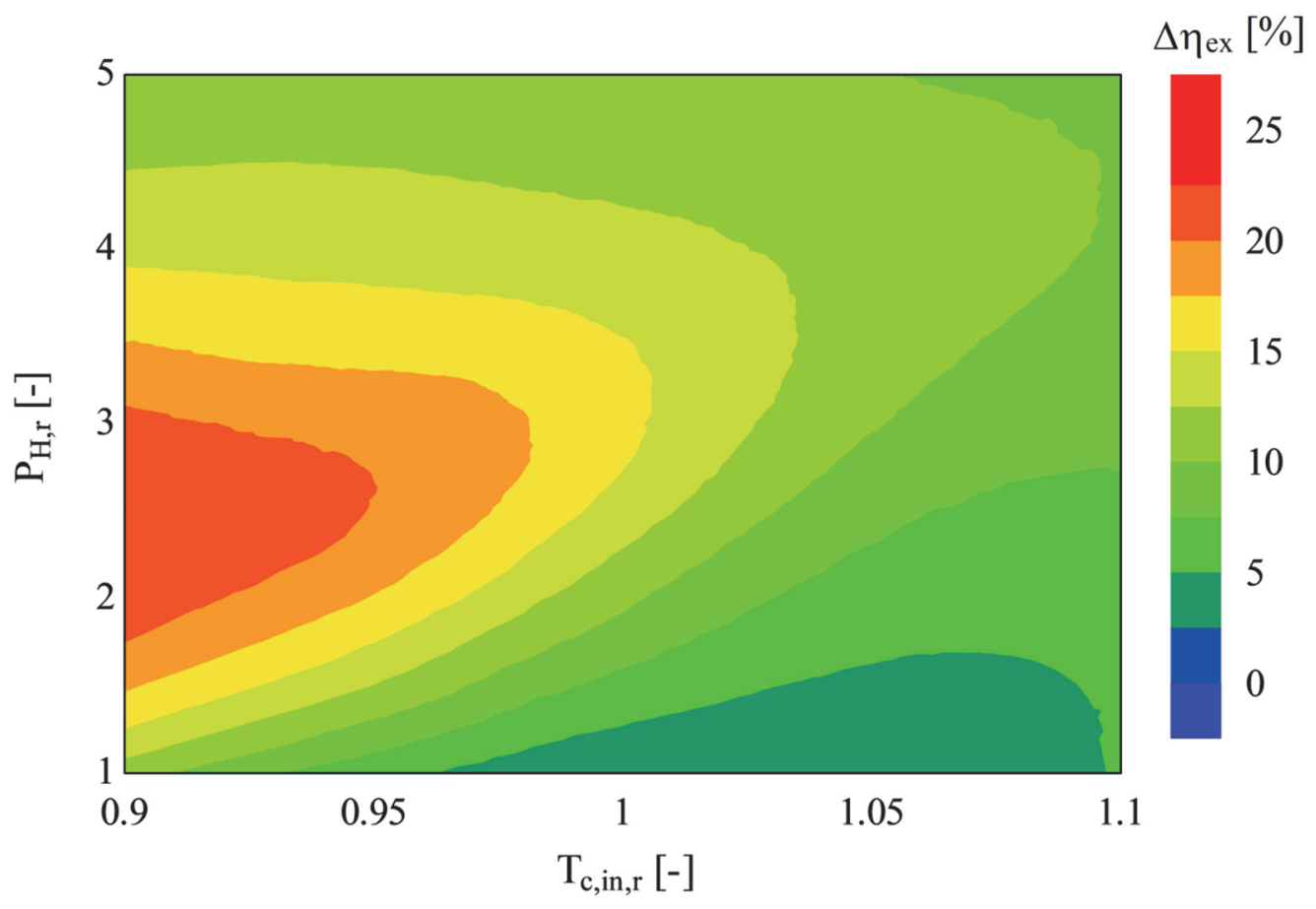

Figure 3.23. Relative increase in exergy efficiency, $\Delta \eta_{e x}$, of supplying an additional heat to a simple cycle as a function of high-pressure side, $P_{H, r}$, and compressor inlet temperature, $T_{c, i n, r}$

The lowest relative increase is obtained around the critical point $\left(T_{c, i n, r}=1\right.$ and $\left.P_{H, r}=1\right)$. These cycles work below the discontinuity line, which means that the advantages of multiheating can barely be used. Increasing the compressor inlet temperature without raising the high pressure $\left(T_{c, i n, r}>1\right.$ and $\left.P_{H, r}=1\right)$ hardly affects the relative increase because the cycle is still working far below the discontinuity line. The solution to take advantage of multi-heating is either raising the high pressure $\left(T_{c, i n, r}=1\right.$ and $\left.P_{H, r}>1\right)$ or decreasing the compressor inlet temperature $\left(T_{c, i n, r}<1\right.$ and $\left.P_{H, r}=1\right)$. In both cases, the purpose is to achieve that the compressor works above the discontinuity line or close to it.

The cycle needs a minimum range of pressures between the high-side pressure and the discontinuity line to take full advantage of multi-heating. This minimum range of pressures corresponds approximately to a pressure ratio $r=2$. The isoexergetic line $\Delta \eta_{e x}=10 \%$ has approximately this pressure ratio. Raising the high-side pressure above this isoexergetic line still increases the relative increase of efficiency up to a maximum. The value of this maximum and its high-side pressure depend on the compressor inlet temperature. The higher 
the compressor inlet temperature the lower the value of the maximum increment $\Delta \eta_{e x}$ and the higher the high-side pressure. The maximum efficiency increment in Figure 3.23 is $\Delta \eta_{e x}=24 \%$ at high-side pressure $P_{H, r}=2.7$ and compressor inlet temperature $T_{c, i n, r}=0.9$.

These results have been obtained with the thermodynamic properties of $\mathrm{CO}_{2}$. Nonetheless, the main trends marked by the discontinuity line are expected to be similar if other fluid is used. Figure 3.24 shows the results obtained with R125. The relative increase in exergy efficiency of supplying an additional heat to a simple cycle is represented as a function of high-pressure side and compressor inlet temperature. Although the values obtained with $\mathrm{CO}_{2}$ in Figure 3.23 are different, the same main trends are maintained with R125.

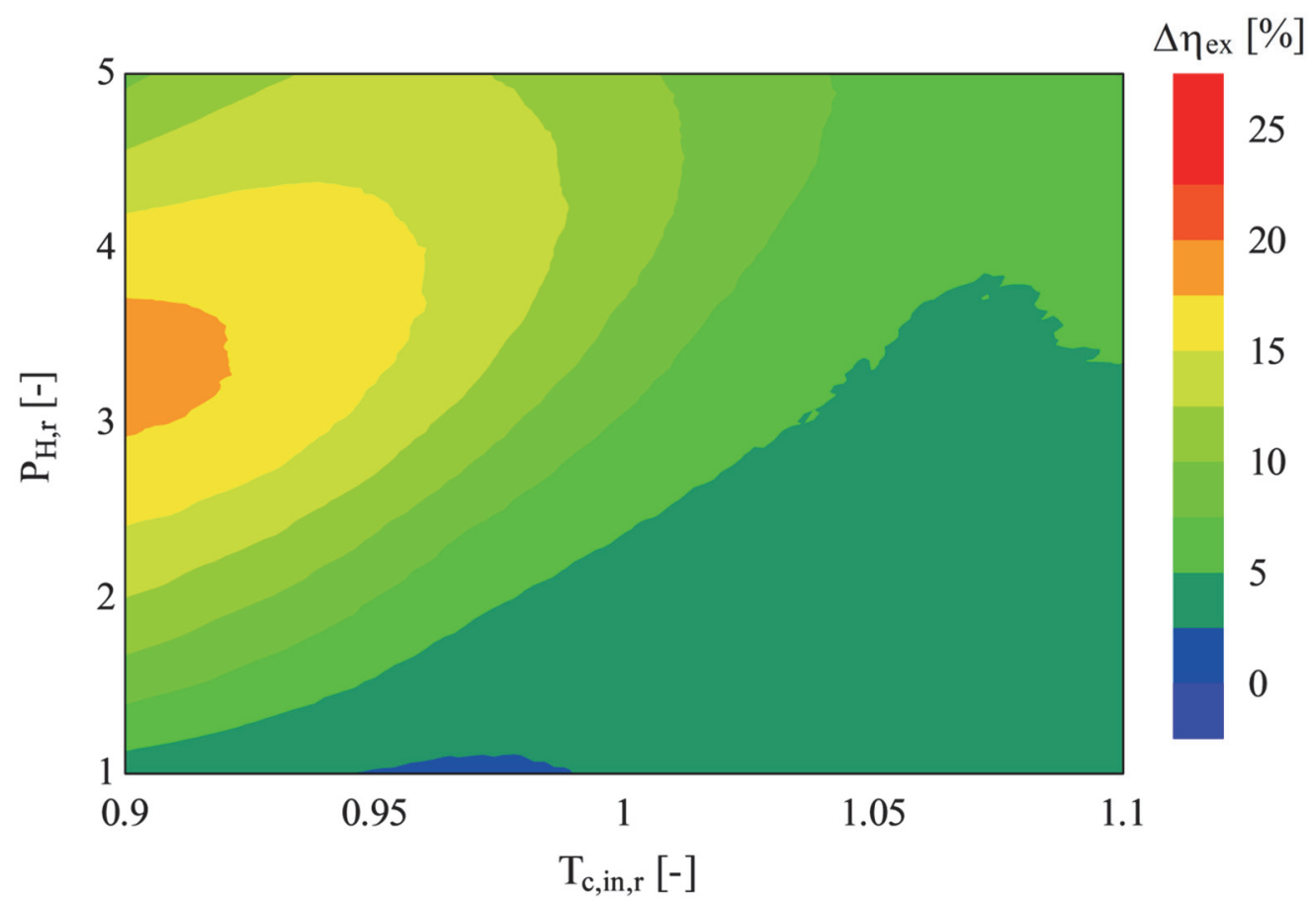

Figure 3.24. Relative increase in exergy efficiency, $\Delta \eta_{e x}$, of supplying an additional heat to a simple cycle with R125 as a function of high-pressure side, $P_{H, r}$, and compressor inlet temperature, $T_{c, i n, r}$

It is important to highlight the importance of the relative increase of efficiency. The limiting values of $T_{c, i n, r}$ and $P_{H, r}$ will be given by the fluid properties and the operational conditions. If the operational conditions are set within a design window, Figure 3.23 and Figure 3.24 show the part of the design window towards where the cycle should move to increase more the efficiency with multi-heating. For example, consider the fluids $\mathrm{CO}_{2}$ and R125 (critical properties in Table 3.3). If the minimum achievable compressor inlet temperature is $T_{c, i n}=50^{\circ} \mathrm{C}$, this would mean $T_{c, i n, r}=1.06$ in the case of $\mathrm{CO}_{2}$ and $T_{c, i n, r}=0.95$ in the case of R125. Both cases take advantage of raising the high-side pressure up to $P_{H, r}=3$. The relative 
increase is $\Delta \eta_{e x}=9 \%$ in the case of $\mathrm{CO}_{2}$ and $\Delta \eta_{e x}=15 \%$ in the case of R125. The lower reduced compressor inlet temperature of R125 allows to achieve a greater relative increase.

Table 3.3. Critical temperature and pressure of fluids $\mathrm{CO}_{2}$ and $\mathrm{R} 125$

\begin{tabular}{lll}
\hline Parameter & $\mathbf{C O}_{\mathbf{2}}$ & $\mathbf{R 1 2 5}$ \\
\hline Critical temperature, $T_{c r}$ & $31^{\circ} \mathrm{C}$ & $66{ }^{\circ} \mathrm{C}$ \\
Critical pressure, $P_{c r}$ & $7.4 \mathrm{MPa}$ & $3.6 \mathrm{MPa}$ \\
\hline
\end{tabular}

For a specific reduced pressure, fluids with higher critical temperature can take more advantage of multi-heating as the higher critical temperature implies lower reduced temperature. The reduced pressure will correspond to different absolute pressure depending on the critical pressure. In the previous case, the reduced high-side pressure $P_{H, r}=3$ corresponds to a pressure $P_{H}=22.2 \mathrm{MPa}$ in the case of $\mathrm{CO}_{2}$ and a pressure $P_{H}=10.8 \mathrm{MPa}$ in the case of R125. If the cycle sets a limit in the high-side pressure, a lower critical pressure allows to increase more the reduced high-side pressure, which gives it a greater potential to increase the cycle efficiency. Raising the high-side pressure increases the cycle efficiency as shown in Figure 3.22, but this does not mean that the relative increase in efficiency of multiheating also does it, as shown in Figure 3.23 and Figure 3.24.

In summary, a low critical pressure gives greater potential to raise the reduced high-side pressure and, therefore, increase the cycle efficiency. On the other hand, a high critical temperature gives greater potential to lower the reduced compressor inlet temperature and, therefore, achieve a greater efficiency with multi-heating.

\subsubsection{Inefficiencies}

Besides the limiting pressures and temperatures, the inefficiencies of cycle components also affect the exergy efficiency. Figure 3.25 shows the influence of the cycle inefficiencies (compressor and turbine efficiency, $\eta_{c}$, and HX effectiveness, $\varepsilon$ ) in the exergetic efficiency, and Figure 3.26 shows the optimum pressure ratio calculated to maximize the exergy efficiency. The results are shown for cycles with compressor inlet temperatures $T_{c, i n, r}=0.95$ (left figures) and $T_{c, i n, r}=1.05$ (right figures), and for simple cycles (top figures) and multiheating cycles with one additional heat (down figures). All the calculations consider a minimum temperature difference in the heat exchangers of $5{ }^{\circ} \mathrm{C}$ in order to avoid the abrupt variations in optimum pressure shown in Figure 2.28 when the effectiveness is close to $\varepsilon=1$. 


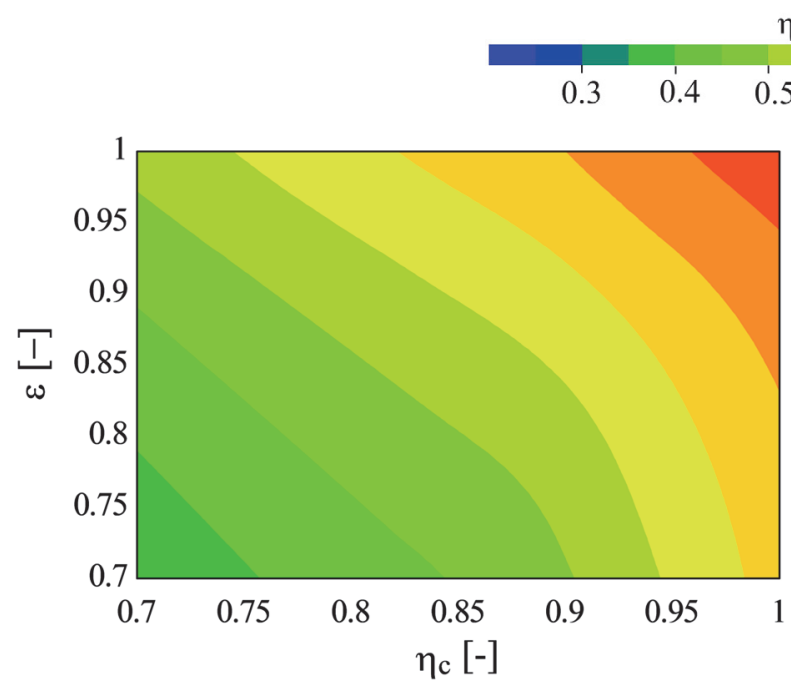

(a) $T_{c, i n, r}=0.95$ and $N_{a h}=0$

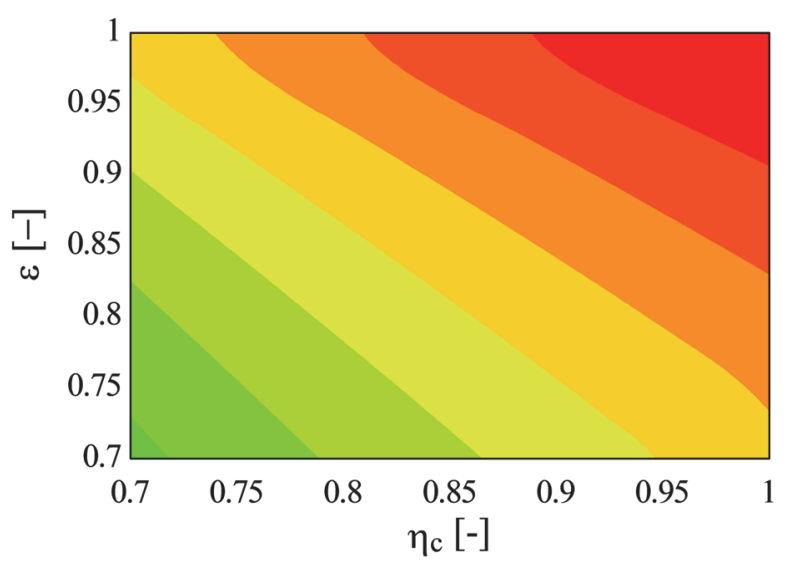

(c) $T_{c, i n, r}=0.95$ and $N_{a h}=1$ $\eta_{\text {ex }}[-]$

$\begin{array}{llll}0.5 & 0.6 & 0.7 & 0.8\end{array}$

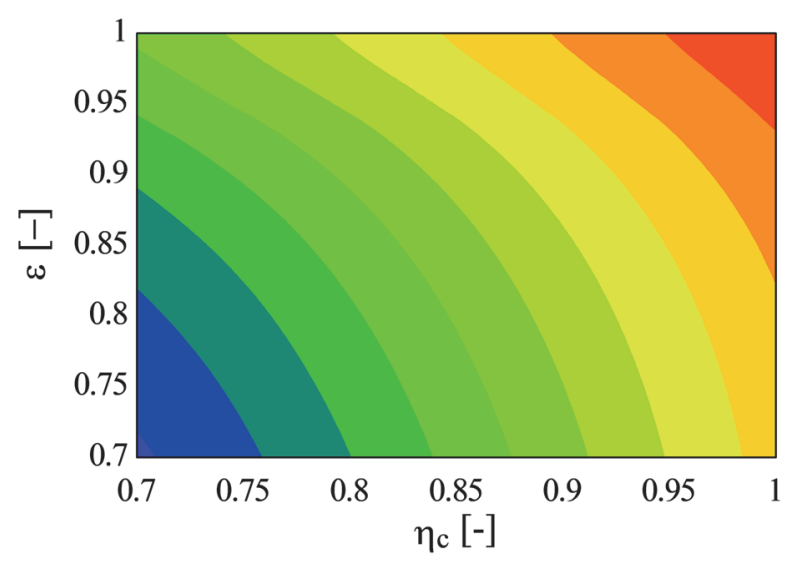

(b) $T_{c, i n, r}=1.05$ and $N_{a h}=0$

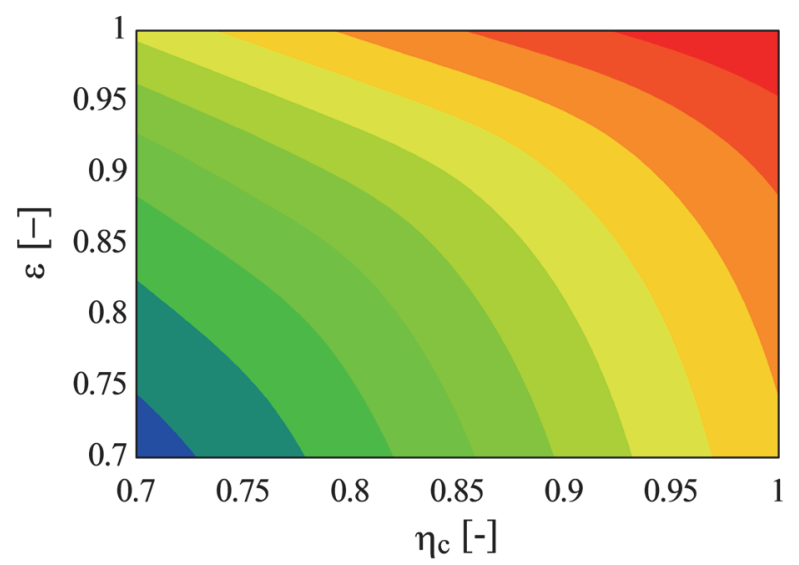

(d) $T_{c, i n, r}=1.05$ and $N_{a h}=1$

Figure 3.25. Exergy efficiency, $\eta_{e x}$, as a function of the compressor inlet efficiency, $\eta_{c}$, and HX effectiveness, $\varepsilon$, in cycles with compressor inlet temperature $T_{c, i n, r}=0.95$ (left figures) and $T_{c, i n, r}=1.05$ (right figures), and no additional heats (top figures) and one additional heat (down figures)

It was seen in previous chapter that, in simple cycles with very high values of $\eta_{c}$ and $\varepsilon$, the exergy efficiencies were very similar regardless of the compressor inlet temperature. This can be appreciated in the right upper corner in the cases with no additional heat $\left(N_{a h}=0\right)$ of Figure 3.25. However, when the values of $\eta_{c}$ and $\varepsilon$ decrease then the exergy efficiency is higher in the case of compressor inlet temperature $T_{c, i n, r}=0.95$. In the cases of multi-heating $\left(N_{a h}=1\right)$, the exergy efficiency is always higher at compressor inlet temperature $T_{c, i n, r}=0.95$ regardless of the component inefficiencies. 


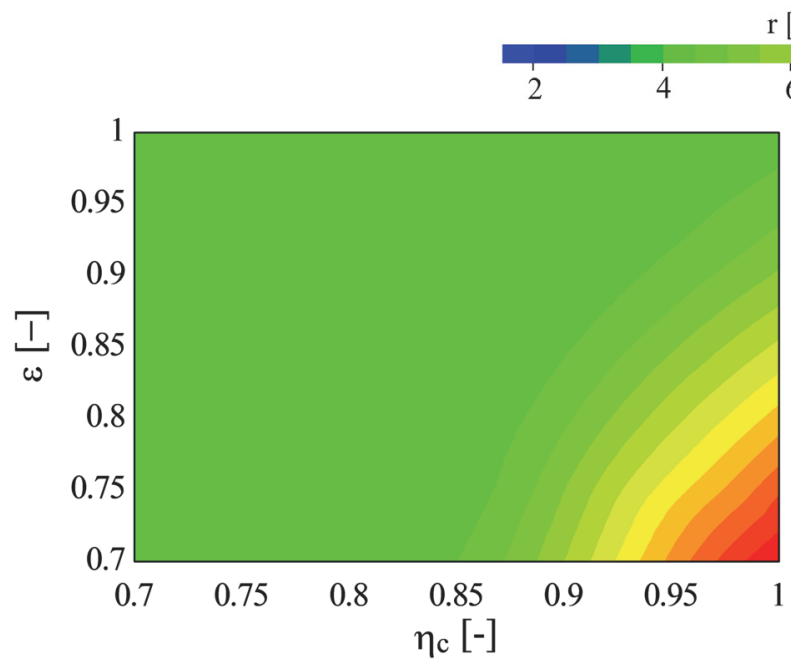

(a) $T_{c, i n, r}=0.95$ and $N_{a h}=0$

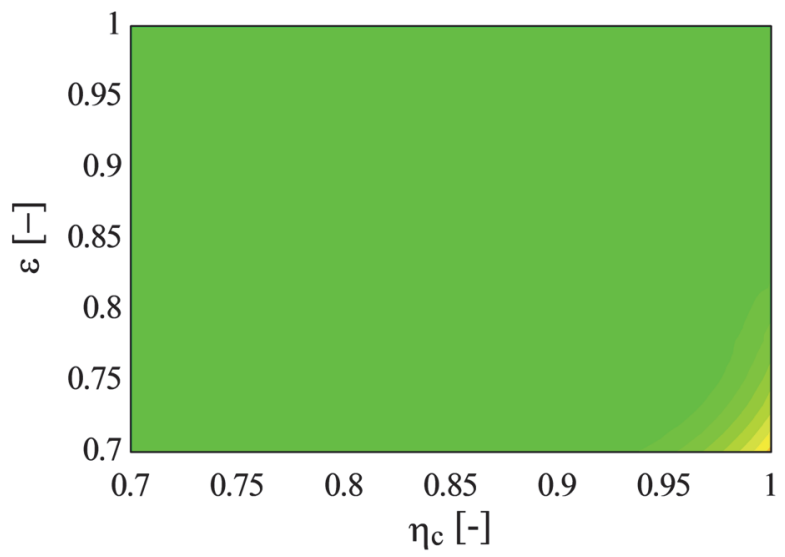

(c) $T_{c, i n, r}=0.95$ and $N_{a h}=1$

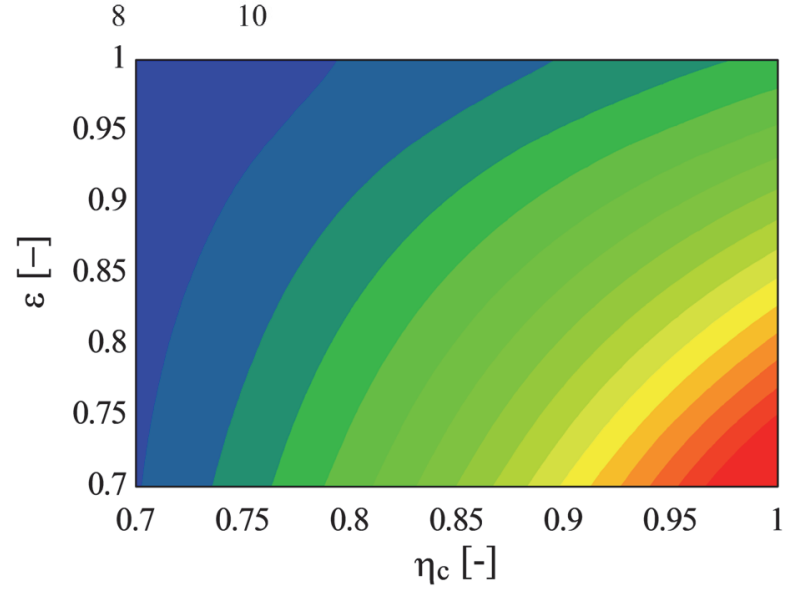

(b) $T_{c, i n, r}=1.05$ and $N_{a h}=0$

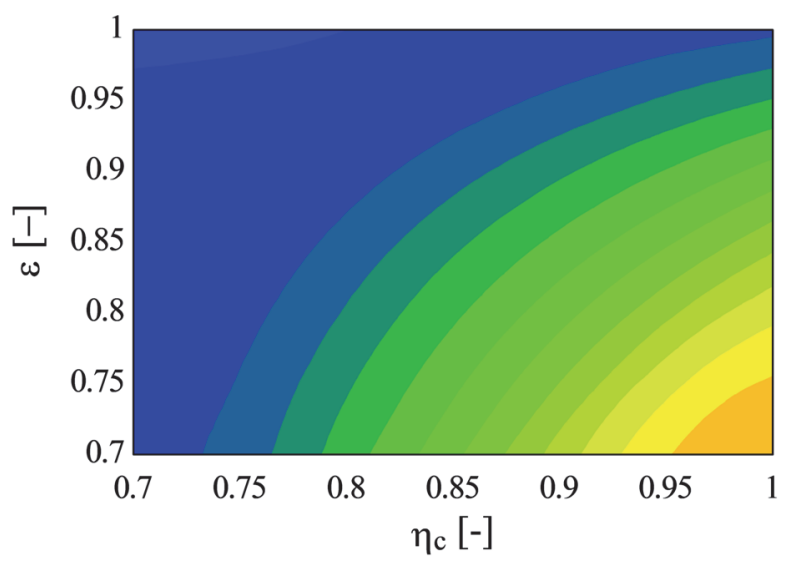

(d) $T_{c, i n, r}=1.05$ and $N_{a h}=1$

Figure 3.26. Optimum pressure ratio, $r$, as a function of the compressor inlet efficiency, $\eta_{c}$, and HX effectiveness, $\varepsilon$, in cycles with compressor inlet temperature $T_{c, i n, r}=0.95$ (left figures) and $T_{c, i n, r}=1.05$ (right figures), and no additional heats (top figures) and one additional heat

(down figures)

Figure 3.26 shows that high turbomachine efficiencies encourage the cycle to use higher pressure ratios to take advantage of the turbomachines. On the other hand, a high effectiveness reduces the optimum pressure ratio. In this case, the cycle benefits more from increasing the heat regeneration. Pericritical cycles with compression inlet temperature $T_{c, i n, r}=0.95$ optimizes the exergetic efficiency with higher pressure ratios than cycles with $T_{c, i n, r}=1.05$. Supplying an additional heat involves lower pressure ratios in both cases due to the smaller influence of the regeneration heat loss in the exergy efficiency.

When the effectiveness is high with respect to the turbomachines efficiency, the optimum low-side pressure tends to be close to the discontinuity line ( $r=4.2$ for $T_{c, i n, r}=0.95$ and $r=2.2$ for $\left.T_{c, i n, r}=1.05\right)$. This tendency is stronger in multi-heating cycles. Even cycles with 
low IHX effectiveness maximize the efficiency at low-side pressures close to the discontinuity line.

The effect of the discontinuity line in the exergy efficiency can be observed in the behaviour of the isoexergetic lines in Figure 3.25. The shape of these isolines can be divided into two regions: one almost vertical and other more inclined. The more inclined part corresponds to the cycles with the low-side pressure working in the discontinuity line. In these cases, effectiveness and turbomachines efficiency affect similarly to the exergy efficiency. In the case of the multi-heating cycle with $N_{a h}=1$ and compressor inlet temperature $T_{c, i n, r}=1.05$ (Figure 3.25d), the inclined isoexergetic lines are almost horizontal. The reasons of the larger influence of the effectiveness in this case are the low pressure ratios in comparison to the case with compressor inlet temperature $T_{c, i n, r}=0.95$ and the extra heat exchanger due to the multiheating. When the pressure ratio is bigger than the corresponding to the discontinuity line then the isoexergetic lines are almost vertical, meaning that the turbomachines efficiency rules the exergy efficiency.

Figure 3.27 analyses the benefit of multi-heating cycles depending on the system inefficiencies. Figure 3.27a and Figure 3.27b show the relative increase of exergy efficiency when one heat is added in a cycle with compressor inlet temperatures $T_{c, i n, r}=0.95$ and $T_{c, i n, r}=1.05$, respectively. The increase of efficiency is higher when the compressor inlet temperature is $T_{c, i n, r}=0.95$ for all the values. The cycles in which the regeneration heat loss has a bigger effect on the efficiency (i.e., cycles with low turbomachines efficiencies and high HX effectiveness) experience greater relative increase of exergetic efficiency. Nevertheless, the effect of the component inefficiencies is different depending on the compressor inlet temperature.

The effectiveness barely affects the increase of efficiency when the compressor inlet temperature is $T_{c, i n, r}=0.95$. This does not mean that increasing the IHX effectiveness does not increment the exergy efficiency, it means that increasing the effectiveness involves a similar increment of efficiency. When the compressor inlet temperature is $T_{c, i n, r}=1.05$, there are two different regions of the effectiveness effect, above and below $\varepsilon=0.9$. In the region above $\varepsilon=0.9$, an increment of effectiveness involves greater increments of exergy efficiency due to the lower optimum pressure ratios of these cycles. 


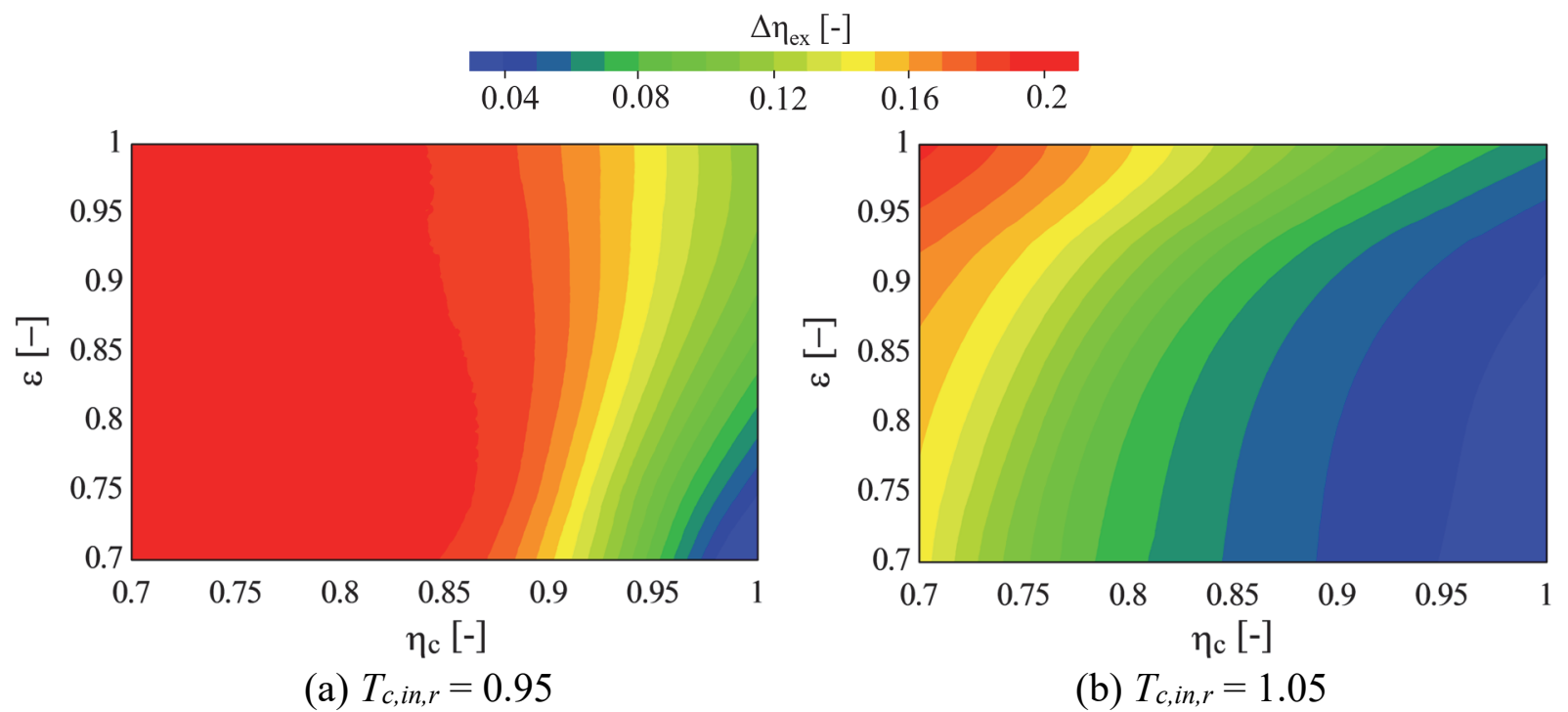

Figure 3.27. Relative increase of exergy efficiency, $\Delta \eta_{e x}$, of supplying one additional as a

function of the compressor inlet efficiency, $\eta_{c}$, and HX effectiveness, $\varepsilon$, in cycles with compressor inlet temperature (a) $T_{c, i n, r}=0.95$ and (b) $T_{c, i n, r}=1.05$

The turbomachines efficiencies have special influence in the relative increase of efficiency. When the compressor inlet temperature is $T_{c, i n, r}=0.95$, these efficiencies rule the increase of exergy efficiency. The relative increase of efficiency in cycles with compressor efficiency lower than $\eta_{c}=0.85$ is the same as the pressure ratio of simple and multi-heating cycles at these compressor efficiencies is the same. When the compressor inlet temperature is $T_{c, i n, r}=1.05$, the turbomachines efficiencies have more influence in the region where the effectiveness is below $\varepsilon=0.9$.

In summary, cycles with low turbomachines efficiencies and high IHX effectiveness take more advantage of multi-heating cycles. The position of the compressor inlet with respect to the discontinuity line establish the relevance of these cycle inefficiencies. The turbomachines efficiencies have special influence in cycles with low compressor inlet temperatures.

\subsection{Summary}

This chapter presents the multi-heating cycle. Two different layouts are proposed to incorporate multi-heating in a cycle. While simple regenerative cycles supply heat by means of a single heat source, multi-heating cycles substitute part of this heat by heats supplied with additional heat sources at lower temperature with the purpose of reducing the strong irreversibilities in the regenerator. The lower exergetic cost of heat sources at lower 
temperatures results in higher exergy efficiencies than simple regenerative cycles. The benefits of multi-heating cycles are analyzed by means of exergy analysis.

The multi-heating cycle characterization is guided with the help of the discontinuity line. The position of the compressor with respect to this line determines the cycle behavior. Cycles with the compressor working in the liquid region take more advantage of multi-heating. The larger irreversibilities in the regeneration of these cycles give a larger potential to improve the exergy efficiency.

The limiting conditions of multi-heating cycles are analyzed together with its relationship with the discontinuity line. The exergy efficiency increases by lowering the compressor inlet temperature and raising the high-pressure side. The high-side pressure must be above the discontinuity line to take full advantage of multi-heating. In the case of multi-heating cycles with one additional heat, the pressure ratio between the high-side pressure and the discontinuity line must be above two to take full advantage of multi-heating. In these cases, the increase of exergy efficiency with respect to a simple cycle is greater than $\Delta \eta_{e x}=10 \%$. This increase may be up to $\Delta \eta_{e x}=24 \%$ if the compressor inlet temperature is lowered and the high-side pressure is raised up to values around $P_{H, r}=3$.

System inefficiencies are also very important in determining the best conditions to take advantage of multi-heating cycles. Multi-heating cycles with low turbomachines efficiencies and high heat exchanger effectiveness obtain larger increases of exergy efficiency. Cycles with low compressor inlet are especially susceptible to the variation of turbomachines efficiencies. For example, in a cycle with compressor inlet reduced temperature $T_{c, i n, r}=0.95$, the exergy efficiency increase of supplying one additional heat may be $\Delta \eta_{e x}=20 \%$ if the turbomachines efficiency is $\eta_{c}=0.85$, or $\Delta \eta_{e x}=5 \%$ if it is close to $\eta_{c}=1$.

The purpose of this chapter has been to characterize the analysis of multi-heating cycles. The compressor position in the thermodynamic diagram determines the benefit of multi-heating cycles with respect to simple regenerative cycles. The tendencies obtained in this chapter with the thermodynamic properties of $\mathrm{CO}_{2}$ can be extrapolated to other fluids. In this way, fluids with higher critical temperature could take more advantage of multi-heating as the compressor inlet reduced temperature could be lower. 


\section{HEAT EXCHANGERS IN MULTI- HEATING CYCLES}

Fluids working in the region close to the critical point experience strong variations of thermodynamic properties. If this variation occurs during the heat regeneration of a pericritical cycle, it involves large irreversibilities that have negative impact in the cycle performance. An option to reduce the negative impact of irreversibilities in the regeneration is the use of multi-heating cycles. These cycles reduce the irreversibilities by means of additional heats that partially substitute the primary heat. Besides the additional-heat sources, the intermediate heat exchangers are the other essential element in multi-heating cycles.

Intermediate heat exchangers have been defined by means of the effectiveness in previous chapters. The use of the effectiveness is very useful to analyse the cycle potential with dimensionless parameters. Several authors use this term to analyse the efficiency of pericritical cycles (Binotti et al., 2017; Padilla et al., 2015a; Rovira et al., 2013). However, the use of the effectiveness may hide the real size of the heat exchangers, which may result in misleading conclusions about the relative economic merit of the cycle (Dyreby et al., 2014). The importance of intermediate heat exchangers in multi-heating cycles makes mandatory to analyse the effect of its size in the cycle performance.

This chapter focuses on the impact of the size of intermediate heat exchangers in simple and multi-heating cycles with $\mathrm{CO}_{2}$. The effect of intermediate heat exchangers on the cycle performance will depend on the definition of it size. Three different ways of defining the HX 
size will be analysed: effectiveness, conductance or volume. The type of heat exchanger chosen to calculate the volume is the Printed Circuit Heat Exchanger (PCHE).

The cycle performance depends on the compressor inlet temperature and, therefore, on the precooler features. Although this chapter focuses on the size of intermediate heat exchangers, the volume of the precooler and its effects on the cycle performance are also analysed for different types of cooling.

\subsection{Thermal size of pericritical cycles}

An important feature in the design of pericritical cycles is the thermal size of each of its phases. The thermal size in the form of specific enthalpy (in $\mathrm{kJ} / \mathrm{kg}$ ) has been implicitly studied in previous results. However, it has not been shown as the main purpose has been to analyse the cycle performance. Figure 4.1 shows the variations of specific enthalpy, $\Delta h$, in each phase of a multi-heating cycle as a function of the compressor inlet $T_{c, i n}$. The results belong to the optimized cycles with one additional heat from Figure 3.21, whose most characteristic feature is the limitation of the high-side pressure to $P_{H, r}=3$. Remember that the Carnot factor is the same in these simulations, which means that reducing the compressor inlet temperature also involves a lower turbine inlet temperature.

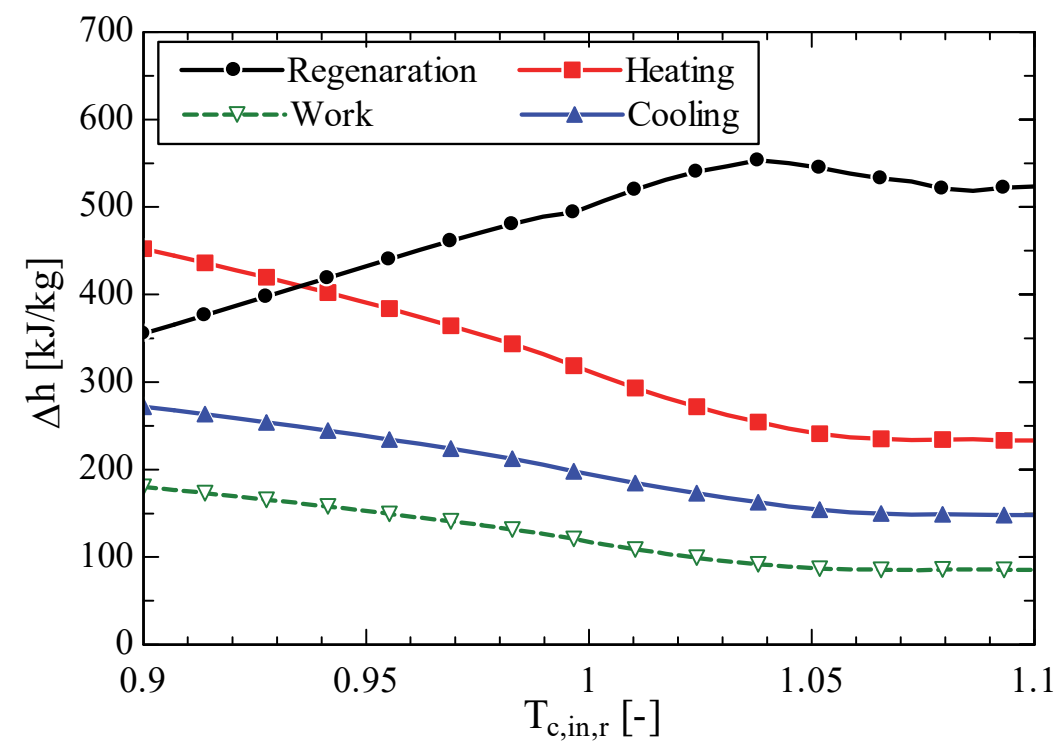

Figure 4.1. Specific enthalpy variation, $\Delta h$, in each phase of a multi-heating cycle as a function of the reduced compressor inlet temperature, $T_{c, i n, r}$

The three phases in which there is heat exchange involved are regeneration, heating (sum of the heat supplied by primary and additional heat sources) and cooling. The specific work is 
also shown in Figure 4.1 although it does not involve heat exchange, but power generation. Cycles with the compressor inlet temperature below $T_{c, i n, r}=1.05$ have the compressor in the liquid region, and the rest in the gaseous region. For temperatures above $T_{c, i n, r}=1.05$ the enthalpy variation is almost constant in all its phases. On the contrary, cycles with the compressor working below temperature $T_{c, i n, r}=1.05$ behave differently depending on the compressor inlet temperature. The lower the compressor inlet temperature, the larger the heating and the cooling are, and therefore, the regeneration requires less energy exchange because most of it is already done by the heat sources and heat sink.

The variation of specific enthalpy gives relevant information of the cycle behaviour. In fact, it defines the power of the different parts of the cycle:

$$
\text { Power }=\dot{m} \cdot \Delta h
$$

This power refers to thermal power when the phase exchanges heat and to electric power in compression and expansion (assuming perfect transmission from mechanical to electric power).

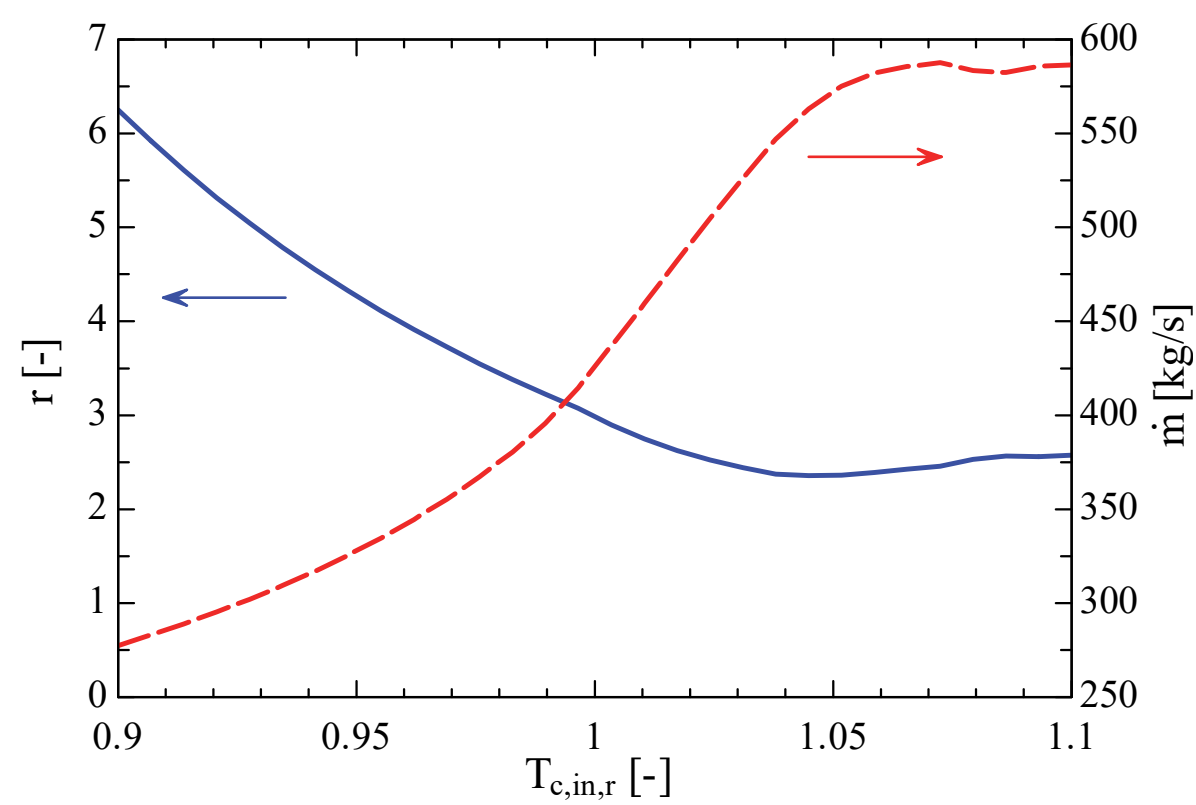

Figure 4.2. Mass flow rate, $\dot{m}$, and pressure ratio, $r$, of a cycle with nominal electric power of $50 \mathrm{MW}$ depending on the compressor inlet temperature, $T_{c, i n, r}$

The maximum efficiency of pericritical cycles has been calculated in previous chapters by means of specific enthalpy variations, i.e., the results shown in Figure 4.1 are independent of the power required in the power plant. Therefore, if the nominal electric power is defined, the mass flow rate is calculated as a result from Equation 4.1. Figure 4.2 shows the mass flow rate, $\dot{m}$, and the pressure ratio, $r$, required to maximize the exergy efficiency of cycles with 
nominal electric power of $50 \mathrm{MW}_{\mathrm{e}}$ depending on the compressor inlet temperature $T_{c, i n}$. Cycles with lower compressor inlet temperatures achieve larger specific work by increasing the pressure ratio, which involves lower mass flow rates to generate $50 \mathrm{MW}$. The result is that the mass flow rate has the opposite tendency to heating and cooling in Figure 4.1, which affects the thermal power of these two cycle phases.

Figure 4.3 shows the thermal power of the heat exchange parts of simple $\left(N_{a h}=0\right)$ and multiheating cycles $\left(N_{a h}=1\right)$ as a function of the compressor inlet temperature, $T_{c, i n, r}$. Heating and cooling thermal powers barely depend on the compressor inlet temperature. However, the regeneration heat does depend on the compressor inlet temperature, and also on the number of additional heats. The regeneration power in the cycles working partially in the liquid region (compressor inlet temperatures below $T_{c, i n, r}=1$ when $N_{a h}=0$ and below $T_{c, i n, r}=1.05$ when $N_{a h}$ $=1$ ) increases with the compressor inlet temperature, and it remains more or less constant when the whole cycle works in the gaseous region. The regeneration power is similar to the heating and cooling thermal powers when the compressor inlet temperatures are low. However, the regeneration power is much greater than the power in the other phases when the compressor inlet temperatures are high.

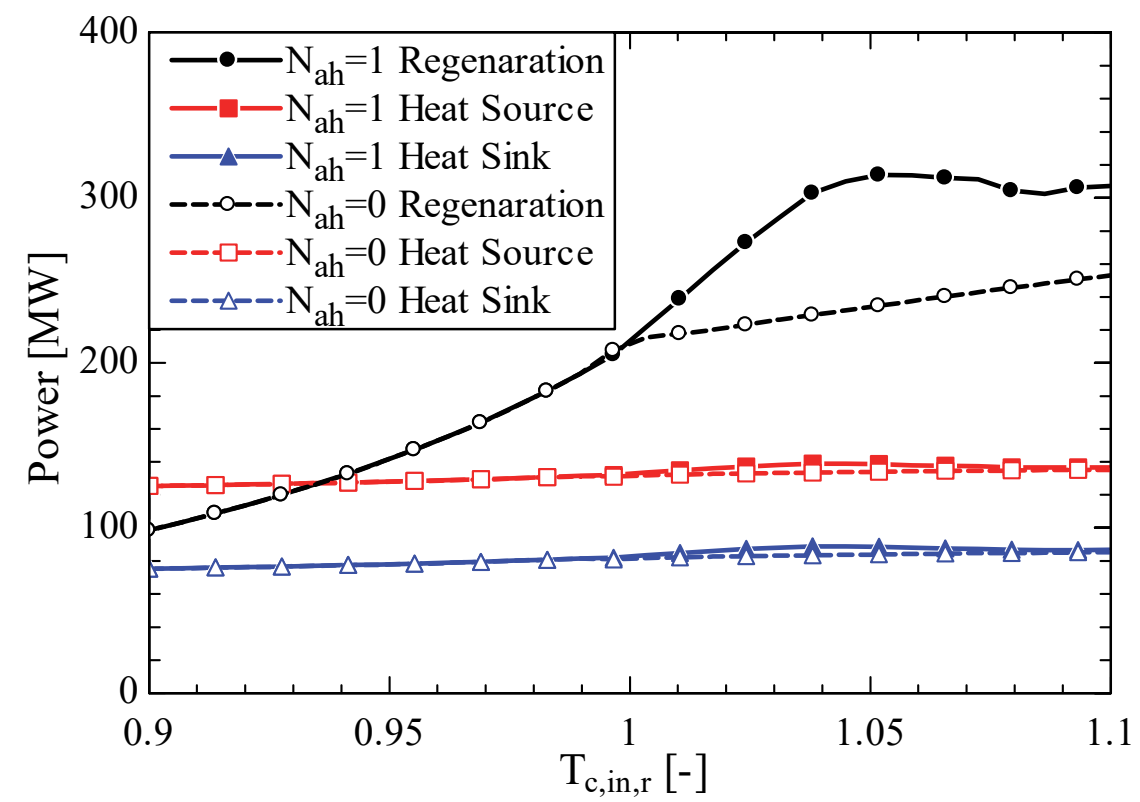

Figure 4.3. Thermal power of the heat exchange parts of a simple $\left(N_{a h}=0\right)$ and multi-heating cycle $\left(N_{a h}=1\right)$ as a function of the compressor inlet temperature, $T_{c, i n, r}$

This chapter focuses on the analysis of pericritical cycles with $\mathrm{CO}_{2}$. Thus, the compressor inlet temperatures will be above $T_{c, i n, r}=1\left(31^{\circ} \mathrm{C}\right)$, as lower temperatures are hardly got when the working fluid is $\mathrm{CO}_{2}$ and the cycle is cooled at ambient conditions. Figure 4.3 shows that 
in cycles with this compressor inlet temperature most of the heat exchange occurs in the regeneration, especially when there is multi-heating $\left(N_{a h}=1\right)$. The following sections will focus mainly on the size of this part of the cycle: the intermediate heat exchangers. It will be of special interest to determine the increase of IHX size in the case of multi-heating cycles with respect to simple cycles.

\subsection{Pericritical cycles with $\mathrm{CO}_{2}$ as working fluid}

Fluids with temperature close to the critical temperature are potential candidates to be the working fluid in pericritical cycles. Figure 2.31 showed some of these candidates. Several instigations stand out $\mathrm{CO}_{2}$ because of its stability and relative inertness (for the temperature range of interest), sufficient knowledge of its thermodynamic properties, non-toxicity, abundance and low cost (Dostal, 2004). As a result of these advantages, pericritical cycles with $\mathrm{CO}_{2}$ as working fluid have been deeply studied during the last decades (Angelino, 1969; Dyreby, 2014; Feher, 1968; Kim, Kim and Favrat, 2012).

Heat exchangers are the largest components in pericritical $\mathrm{CO}_{2}$ cycles (Dostal, 2004). Thereupon, several authors analyse the impact of the heat exchangers size in the cycle performance (Bryant, Saari and Zanganeh, 2011; Dostal, 2004; Dyreby, 2014; Neises and Turchi, 2014). This chapter analyses the influence of the heat exchangers in the exergy efficiency of simple and multi-heating pericritical cycles with $\mathrm{CO}_{2}$. Table 4.1 shows a summary of the parameters used to define these cycles.

Table 4.1. Parameters of pericritical $\mathrm{CO}_{2}$ cycle

\begin{tabular}{ll}
\hline Parameter & Value \\
\hline Maximum high-side pressure, $P_{H}$ & $25 \mathrm{MPa}$ \\
Compressor inlet temperature, $T_{c, \text { in }}$ & $30-50^{\circ} \mathrm{C}$ \\
Turbine inlet temperature, $T_{t, i n}$ & $700^{\circ} \mathrm{C}$ \\
Compressor efficiency, $\eta_{c}$ & 0.89 \\
Turbine efficiency, $\eta_{t}$ & 0.93 \\
Pressure drop in the primary heat exchanger, $\chi$ & 0.05 \\
Net power & $50 \mathrm{MWe}$
\end{tabular}


The compressor inlet temperature mainly depends on the ambient temperature and the fluid used to cool down the $\mathrm{CO}_{2}$ in the precooler. The wet- and dry-bulb temperatures $20^{\circ} \mathrm{C}$ and $40^{\circ} \mathrm{C}$, respectively, are representative of ambient temperatures for wet- and dry- cooling methods near Almería (Spain) in summer (EnergyPlus, 2018). Assuming a $10{ }^{\circ} \mathrm{C}$ pinch point temperature difference in the heat exchangers, compressor inlet temperatures are set to $30{ }^{\circ} \mathrm{C}$ and $50^{\circ} \mathrm{C}$ for wet- and dry-cooled cycles, respectively (Dunham and Iverson, 2014).

Several authors set the minimum compressor inlet temperature to $32^{\circ} \mathrm{C}$ in order to be above the critical temperature (Dostal, 2004; Dyreby, 2014; Sarkar, 2009), and avoid phase change and/or the strong property variations characteristic of the critical point. However, it is not the critical temperature itself but the discontinuity line which defines the strong variation of properties. Cycles with the temperature above the critical temperature may also cross the discontinuity line, resulting in strong property variations especially in the precooler. Therefore, from the discontinuity line point of view, decreasing the compressor inlet temperature from $32{ }^{\circ} \mathrm{C}$ to $30^{\circ} \mathrm{C}$ only implies stronger property changes, but strong in both cases. Decreasing this temperature from $34^{\circ} \mathrm{C}$ to $32^{\circ} \mathrm{C}$ would also imply a similar variation, however, the point of view of phase change makes these two cases seem very different. This work analyses the compressor inlet temperature $\mathrm{T}=30^{\circ} \mathrm{C}$ to emphasizes the concept of pericritical cycle, which avoids distinguishing from cycles with or without phase change.

The maximum temperature of the cycle is set to $700{ }^{\circ} \mathrm{C}$. Carbon Dioxide can reach these temperatures without degradation problems (Invernizzi, 2017). Thermal power plants such as Concentrating Solar Power plants are expected to operate at these temperatures (and even above $700^{\circ} \mathrm{C}$ ) in the near future (Mehos et al., 2017).

The reduced temperatures corresponding to $30^{\circ} \mathrm{C}$ and $50^{\circ} \mathrm{C}$ are 0.997 and 1.063 , respectively. Figure 3.23 showed in previous chapter that, in this range of temperatures, the largest potential for multi-heating cycles is between reduced high-side pressures $P_{H, r}=3$ and $P_{H, r}=3.5$. According to this, the high-side pressure is set to $P_{H}=25 \mathrm{MPa}$, which corresponds to a reduced pressure $P_{H, r}=3.4$. This pressure is commonly used to analyse pericritical $\mathrm{CO}_{2}$ cycles due to the higher performance improvements with respect to lower pressures (Dunham and Iverson, 2014; Dyreby, 2014).

Pressure drop during the heating will depend on the type of power plant. For example, investigations about solar thermal power plants report pressures drops between close to 0 bar and 13.4 bar, depending on the solar receiver (Khivsara et al., 2014; Pye et al., 2014). For the case of $P_{H}=25 \mathrm{MPa}$, these pressure drops correspond to $\chi=0-6.1 \%$. A pressure drop $\chi=5 \%$ 
will be considered for the primary heat source. Even in the cycles with more than one heat, the pressure drop will be considered only in the primary heat because this pressure drop will be the largest one. The pressure drop in the rest of components is considered negligible as the main pressure drop in these cycles happens in the primary heat exchanger (Padilla et al., 2015b). Only the last section of this chapter will consider the pressure drop in the intermediate heat exchanger and precooler.

The turbomachines performance will be simulated by means of the isentropic efficiency, defined in Equations 2.82 and 2.83. Compressor and turbine efficiencies values of 0.89 and 0.93, respectively, are common values used in the simulation of this type of cycles (Dostal, 2004; Dyreby, 2014; Turchi et al., 2012; Wang, He and Zhu, 2017).

Table 4.1 does not include heat exchange parameters, which are needed to complete the cycle definition. Next section analyses different methods to determine the heat exchange in the cycle.

\subsection{Heat exchanger models}

According to Dostal (2004), heat exchangers are by far the largest components in cycles working in the conditions of Table 4.1. Therefore, it is necessary to pay especial attention to the design of these components. This chapter focuses mainly, although not uniquely, on the design of intermediate heat exchangers. The reason is that one of the novelties of this work is the analysis of multi-heating cycles, whose design highly depends on the intermediate heat exchangers. Moreover, Figure 4.3 has shown that the regeneration represents the biggest thermal power in cycles with reduced compressor inlet temperatures between $T_{c, i n, r}=0.95$ and $T_{c, i n, r}=1.1$.

The performance of heat exchangers in thermodynamic cycles is commonly represented by the effectiveness. The use of this parameter allows to characterize the cycle potential as a function of a dimensionless number. However, a disadvantage of characterizing the performance of heat exchangers with the effectiveness is that identical values of effectiveness under different conditions may result in heat exchangers of very different sizes.

Researchers from National Renewable Energy Laboratory (NREL) prefer to use the heat exchanger conductance in their analysis of supercritical cycles (Neises and Turchi, 2014). The conductance defines the thermal size of a heat exchanger. In this way, an estimation of the heat exchanger size can be obtained without the need of carrying out a real design, which is 
very useful to compare different types of cycles such as simple regenerative cycles and multiheating cycles. However, two heat exchangers with the same conductance may have different real size depending on its design and the heat transfer conditions. Thus, a more rigorous analysis will compare the real size by means of the volume. The problem is that the heat exchanger model adds additional variables to the analysis, which may result into conclusions valid only for specific cases.

The purpose of this chapter is to analyse the different methods used to define the heat exchangers and its implication in the cycle performance. The cycle performance will be analysed with heat exchangers defined by effectiveness, conductance and volume.

\subsubsection{Thermal conductance}

The heat exchangers are modelled assuming a counter-flow configuration. The conductance, $U A$, of these heat exchangers is defined as the ratio between the exchanged thermal power, $\dot{Q}$, and the logarithmic mean temperature difference, $\Delta T_{l m}$ (Incropera et al., 2007):

$$
U A=\frac{\dot{Q}}{\Delta T_{l m}}
$$

Where the logarithmic mean temperature difference is:

$$
\Delta T_{l m}=\frac{\left(T_{\text {in,hot }}-T_{\text {in }, \text { cold }}\right)-\left(T_{\text {out }, \text { hot }}-T_{\text {out }, \text { cold }}\right)}{\ln \frac{T_{\text {in }, \text { hot }}-T_{\text {in }, \text { cold }}}{T_{\text {out }, \text { hot }}-T_{\text {out }, \text { cold }}}}
$$

Equation 4.2 defines the thermal conductance of a heat exchanger with constant fluid properties. However, the fluid in pericritical cycles have strong property variations. Thus, in order to accurately capture the effects of changing properties, the heat exchanger must be

\begin{tabular}{|c|c|c|c|c|c|c|c|c|}
\hline $\begin{array}{l}\text { cold in } \longrightarrow \\
\text { hot out } \longrightarrow\end{array}$ & 1 & 2 & $\ldots$ & i-1 & i & $\mathrm{i}+1$ & $\ldots$ & $\mathrm{N}_{\mathrm{HX}}$ \\
\hline
\end{tabular}
discretized into $N_{H X}$ sub-heat exchangers connected in series as shown in Figure 4.4.

Figure 4.4. Heat exchanger discretization

The rate of heat transfer of each sub-heat exchanger $i$ will be $\dot{Q}_{i}$ :

$$
\dot{Q}_{i}=\frac{\dot{Q}}{N_{H X}}
$$


And the conductance of each sub-heat exchanger, $U A_{i}$ :

$$
U A_{i}=\frac{\dot{Q}_{i}}{\Delta T_{l m, i}}
$$

The sum of sub-heat exchanger conductances will be the total conductance, $U A$ :

$$
U A=\sum_{i=1}^{N_{H X}} U A_{i}
$$

The number of sub-heat exchangers used for the calculations will be above $N_{H X}=30$, which has been proved to provide mesh independent results. In the case of the regenerator, if it has more than one heat exchanger, the total conductance will be the sum of conductances of each heat exchanger.

Figure 4.5a shows the temperature evolution in a regenerator and Figure $4.5 \mathrm{~b}$ the conductance of its $N_{H X}=40$ sub-heat exchangers. The regenerator belongs to two cycles optimized in previous chapter: the black lines correspond to a simple regenerative cycle $\left(N_{a h}=0\right)$ and the red lines to a multi-heating cycle with $N_{a h}=1$. In the simple cycle, the conductance of the sub-heat exchangers decreases along the regeneration due to the increase of temperature difference between the low- and high-pressure sides. In the case of supplying one additional heat, the sub-heat exchanger conductances are equal before the additional heat, but the lower temperature difference after the additional heat involves larger conductances, which will be reduced as the temperature difference increases.

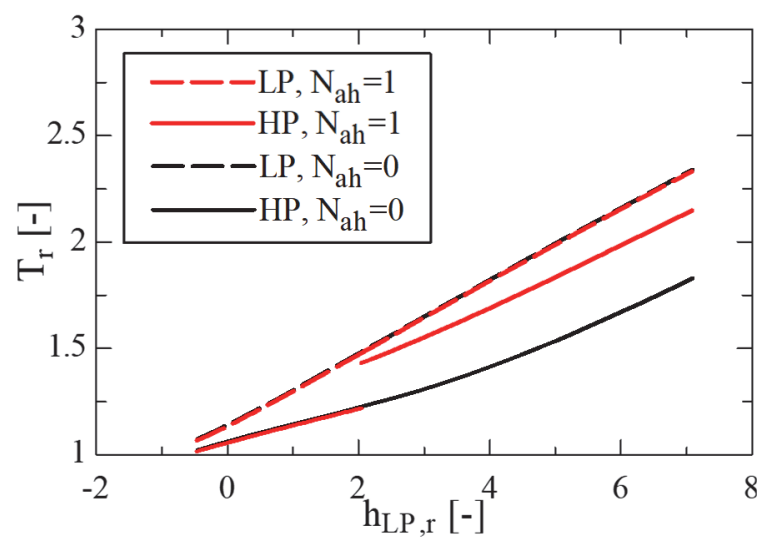

(a) Temperature evolution

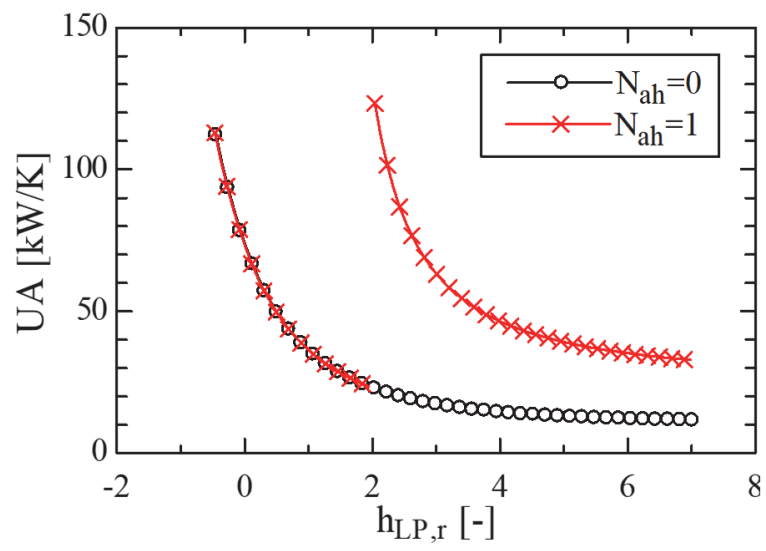

(b) Conductances

Figure 4.5. (a) Reduced temperatures, $T_{r}$, and (b) conductances, $U A$, of $N_{H X}=40$ sub-heat exchangers distributed along the regeneration of a simple $\left(N_{a h}=0\right)$ and a multi-heating cycle with $N_{a h}=1$ 
The results shown in Figure 4.5 belong to a cycle with global effectiveness $\varepsilon=0.95$. The total conductance needed in the simple regenerative cycle to satisfy this condition is $U A=2100 \mathrm{~kW} / \mathrm{K}$. The regeneration in the multi-heating cycle is defined by a global effectiveness and an HTR effectiveness with the same value. The total conductance to satisfy this regeneration is $U A=3968 \mathrm{~kW} / \mathrm{K}$ (sum of $U A_{L T R}=1329 \mathrm{~kW} / \mathrm{K}$ and $U A H T R=2640 \mathrm{~kW} / \mathrm{K}$ ). Both simple and multi-heating cycles have the same enthalpy variation in the cooling and the heating, but the regenerator conductance increases when an additional heat is supplied.

Previous chapters have defined heat exchangers by means of the effectiveness. This chapter allows to define it by means of its conductance. The method to simulate a cycle as a function of conductance will be an iterative process. First, the cycle will be simulated with an initial effectiveness to define the heat exchanger. The resulting conductance will be compared with the defined one. If the resulting conductance is lower than the defined one then the effectiveness of the next iteration is increased, otherwise it is decreased. This iterative process is repeated until the difference between the defined and the resulting conductances is lower than $0.1 \%$.

In simple cycles defined with the conductance method, the regeneration will be defined by the conductance $U A$. In multi-heating cycles with one additional heat, the regeneration will be defined by the additional-heat temperature, $T_{a h}$, the total conductance (sum of LTR and HTR conductances) $U A$, and the LTR fraction, $x_{L T R}$, defined as:

$$
x_{L T R}=\frac{U A_{L T R}}{U A}=\frac{U A_{L T R}}{U A_{H T R}+U A_{L T R}}
$$

Defining the conductance is a common method to represent the thermal size of heat exchangers. The advantage of this parameter is to avoid the real design of the heat exchangers in the cycle analysis, which would add more variables and could hinder some conclusions. However, there are different factors that the conductance does not consider such as the heat transfer behavior and the pressure drops. Next section presents a model to calculate the size of heat exchangers in pericritical cycles.

\subsubsection{Printed Circuit Heat Exchanger design}

The first step to design a heat exchanger is to choose the type of heat exchanger. Classical shell and tube heat exchangers are not suitable for pericritical $\mathrm{CO}_{2}$ cycles (Dostal, 2004). These cycles need compact heat exchangers due to the large thermal power needed during the regeneration. The small tube diameters needed to achieve this compactness with shell and 
tube heat exchangers would lead to difficulties in the manufacture. Moreover, the highpressure difference in the regeneration would involve thick tube walls. Therefore, shell and tube heat exchangers are discarded in the analysis of pericritical $\mathrm{CO}_{2}$ cycles.

Dostal (2004) investigated two different compact heat exchanger types: plate and fin compact heat exchangers and Printed Circuit Heat Exchangers (PCHE). The high-pressure differential involved too big sizes in the former ones, so PCHEs were selected. A PCHE consists of plates into which semi-circular channels are chemically etched. The plates are placed one on top of each other in hot plate / cold plate sequence with counter-current flow. All the plates are diffusion bonded forming a monolithic block. Figure 4.6 shows a scheme of a PCHE crosssection.

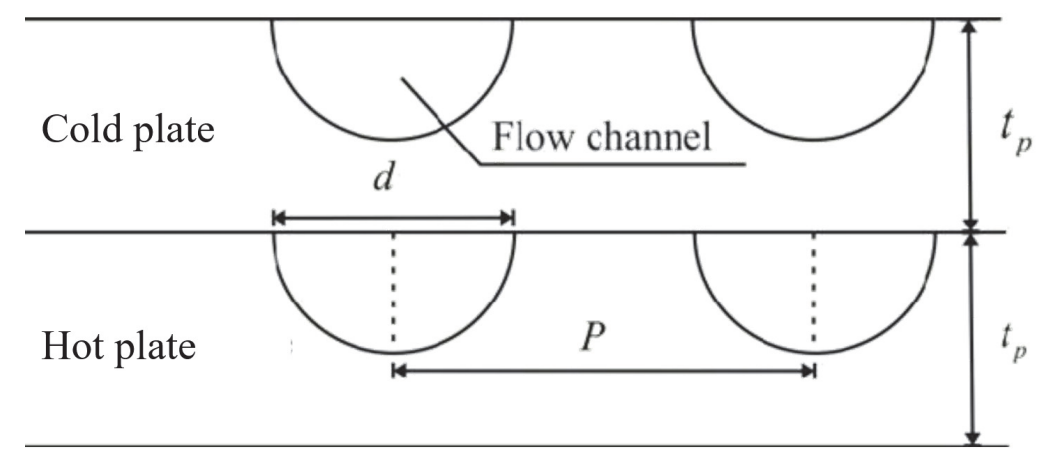

Figure 4.6. Scheme of a PCHE cross-section (Chen et al., 2013)

The model of a PCHE is developed in the commercial software EES (Klein, 1992). The parameters used in the model are presented in Table 3.1. The conductivity, $k_{s s}$, refers to the material that shapes the plates. The rest of the parameters refer to dimensions from Figure 4.6. These parameters were proposed by Dostal (Dostal, 2004), and belong to PCHEs from the company Heatric (www.heatric.com).

Table 4.2. PCHE parameters

\begin{tabular}{lc}
\hline Parameter & Value \\
\hline Semi-circular channel diameter, $d$ & $2 \mathrm{~mm}$ \\
Plate thickness, $t_{p}$ & $1.6 \mathrm{~mm}$ \\
Pitch, $P$ & $2.44 \mathrm{~mm}$ \\
SS304 conductivity, $k_{S S}$ & $23 \mathrm{~W} / \mathrm{m}-\mathrm{K}$
\end{tabular}


Modules fabricated by the company Heatric have specific size, which means that the connection of them in series and parallel would give the total volume. The model developed in EES assumes the PCHE as a single piece. Although this could lead to think that the calculated volume could be underestimated, validations with models considering the connections of smaller PCHEs have shown very similar results (Gezelius, 2004).

The volume of a PCHE will be calculated as the total transversal area, Atr, times the total length, $L$ :

$$
\text { Vol }=A_{t r} L
$$

The transversal area will be an input in the model, and the length, together with the volume, an output. The transversal area will determine the total number of channels in the PCHE, $N_{c h, t o t a l}$, by means of Equation 4.9 .

$$
A_{t r}=N_{c h, t o t a l} A_{s q}
$$

where $A_{s q}$ is the square area occupied by each channel and the corresponding part of the wall to have symmetric channels:

$$
A_{s q}=t_{p} P
$$

The PCHE model considers that all hot and cold channels are identical. Therefore, the model will simulate one hot channel and one cold channel and extend its behaviour to the rest of the channels. Since the geometry is the same in the hot and cold channels, there will be the same number of channels on both sides:

$$
N_{c h, h o t}=N_{c h, \text { cold }}=\frac{N_{c h, t o t a l}}{2}
$$

The PCHE will be divided into $N_{H X}$ sub-heat exchangers in the same way than the conductance in Figure 4.4. The thermal conductance, $U A$, of each sub-heat exchanger, $i$, was calculated in Equation 4.5. From this equation, if the PCHE had just one hot channel and one cold channel then the length of the sub-heat exchanger channels, $l_{i}$, would be:

$$
l_{i}=\frac{\dot{Q}_{i}}{U_{i} \cdot \operatorname{per} \cdot \Delta T_{l m}}=\frac{U A_{i}}{U_{i} \cdot p e r}
$$

where $U_{i}$ is the overall heat transfer coefficient and per the perimeter of each channel, defined as: 


$$
\text { per }=\frac{\pi d}{2}+d
$$

The logarithmic mean temperature difference, $\Delta T_{l m}$, and the rate of heat exchange, $\dot{Q}_{i}$, in each sub-heat exchanger $i$ was calculated in previous section. The overall heat transfer coefficient, $U_{i}$, can be approximated with Equation 4.14 (Dostal, 2004):

$$
U_{i}=\frac{1}{\frac{1}{h t c_{\text {cold }, i}}+\frac{k_{S S}}{t}+\frac{1}{h t c_{\text {hot }, i}}}
$$

where $h t c_{c o l d}$ and $h t t_{\text {chot }}$ are the convection heat transfer coefficients on cold and hot sides respectively, and $k_{S S}$ is the thermal conductivity of the PCHE material. The convection heat transfer coefficients, $h t c$, are calculated by means of semi-empirical correlations following the models of Dostal (2004) and Gezelius (2004). These correlations can be found in Appendix B. If the PCHE had $N_{c h, h o t}$ channels in the hot side (and so the same number in the cold side) then the length of each sub-heat exchanger channel, $L_{i}$, would be:

$$
L_{i}=\frac{l_{i}}{N_{c h, h o t}}
$$

The sum of the lengths of each sub-heat exchanger $i$ will the total HX length, $L$ :

$$
L=\sum_{i=1}^{N_{H X}} L_{i}
$$

This length together with the transversal area allows the calculation of the volume by means of Equation 4.8.

Figure 4.7 shows an example of the volume of a heat exchanger with conductance $U A=4050 \mathrm{~kW} / \mathrm{K}$ as a function of the total number of channels (proportional to the transversal area). The smaller the transversal area, the greater the velocity needed in each PCHE channel is. Greater velocities involve greater heat transfer coefficients, which means that smaller heat transfer areas (and therefore smaller volumes) are needed to cover the conductance. However, greater velocities also involve higher pressure drops. The minimum heat exchanger size will have the minimum number of channels that allow the limits of pressure drop.

The pressure losses are separated into form losses and friction losses. The form losses are two, the entrance and the exit loss. The expression for these losses is: 


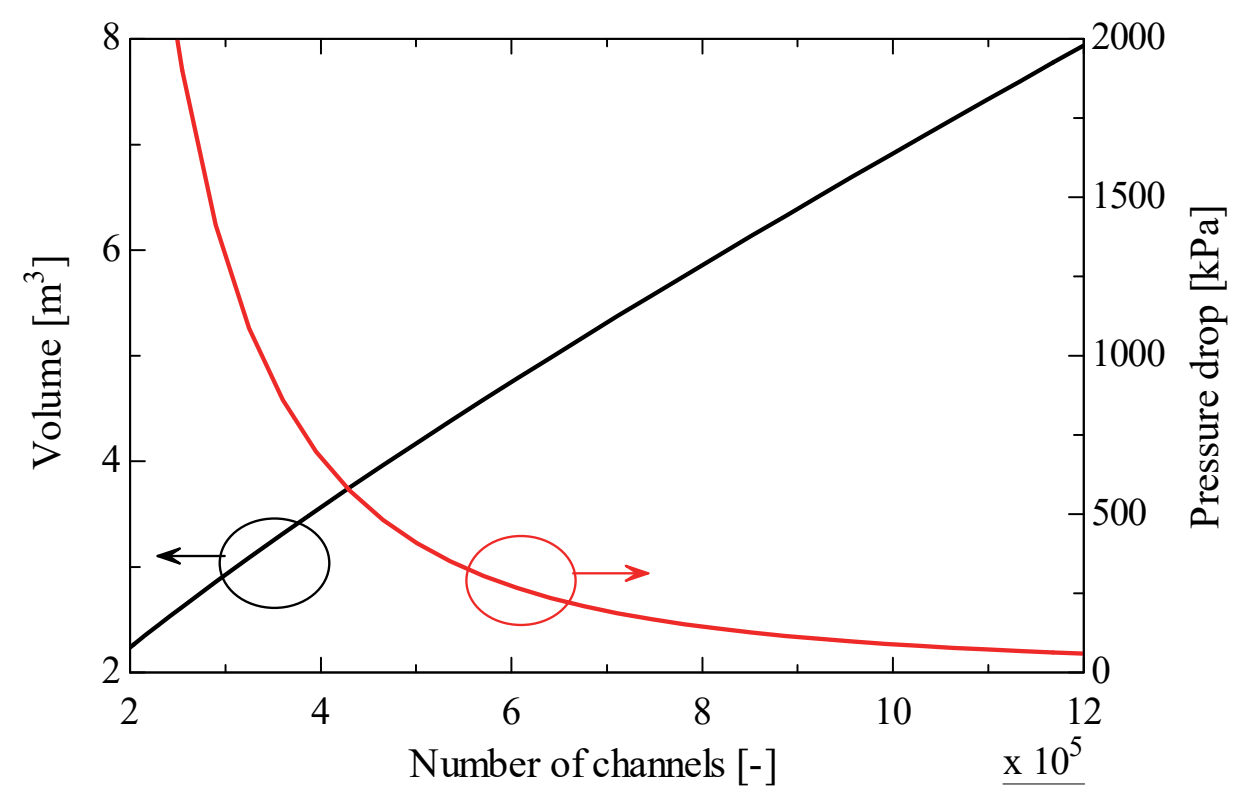

Figure 4.7. Volume and pressure drop of a PCHE with conductance $U A=4050 \mathrm{~kW} / \mathrm{K}$ as a function of the total number of channels

$$
\Delta P=K \rho \frac{v^{2}}{2}
$$

where $K$ is the form loss coefficient ( 0.5 for the entrance loss and 1 for the exit loss), $\rho$ is the local fluid density and $v$ the local fluid velocity.

The friction losses are calculated for each sub-heat exchanger, $i$, as:

$$
\Delta P_{i}=f_{i} \frac{L_{i}}{d_{h}} \rho_{i} \frac{v_{i}^{2}}{2}
$$

where $d_{h}$ is the hydraulic diameter of the semi-circular channel and $f_{i}$ the local friction factor.

The friction factor is calculated by means of semi-empirical correlations following the models of Dostal (2004) and Gezelius (2004). These correlations can be found in Appendix B. The model developed to calculate the PCHE volume and pressure drops is validated with results from Dostal (2004) and Gezelius (2004).

The volume of a PCHE can be reduced at the expense of increasing the pressure drop as shown in Figure 4.7. The pressure drop in each PCHE of the regeneration phase will be limited to $\Delta P_{I H X}=200 \mathrm{kPa}$ distributed between the high- and low-pressure sides. Nonetheless the results show that most of the pressure drop (around 80\%) happens in the low-pressure side. 
The PCHE model will also be used to estimate the volume of the precooler once the rest of the cycle is defined. In the precooler, the maximum pressure drop will be limited to $\Delta P_{p c}=100 \mathrm{kPa}$ in the $\mathrm{CO}_{2}$ side. The pressure drop in the other side of the PCHE, $\Delta P_{c o o l}$, will be limited by means of a maximum pumping power, $\dot{W}_{p}$, defined as:

$$
\dot{W}_{p}=\frac{\dot{m}_{c o o l}}{\rho} \Delta P_{c o o l}
$$

The optimum volume of the precooler will be the result of varying number of channels and mass flow rate of the cooling fluid in an iterative process.

The limiting pumping power in wet-cooled cycles is set to $1 \%$ of the cycle power. The size of the precooler in dry-cooled cycles with this limiting value resulted in immense volumes due to the bad properties of air to exchange heat. Thus, the pumping power is limited to $5 \%$ in dry-cooled cycles. Table 4.3 shows a summary of the limiting values set to pressure drops in the PCHE model.

Table 4.3. Pressure drop limitations in the design of PCHEs

\begin{tabular}{lc}
\hline Parameter & Value \\
\hline Pressure drop heat exchanger in regeneration, $\Delta P_{I H X}$ & $200 \mathrm{kPa}$ \\
Pressure drop $\mathrm{CO}_{2}$ in precooler, $\Delta P_{p c}$ & $100 \mathrm{kPa}$ \\
Pumping power in wet-cooled cycles & $0.5 \mathrm{MW}$ \\
Pumping power in dry-cooled cycles & $2.5 \mathrm{MW}$ \\
\hline
\end{tabular}

\subsubsection{Heat transfer model}

The model used for the calculations of heat transfer coefficient is detailed in Appendix B. Nevertheless, some annotations must be made about its applicability and its implications.

The heat transfer coefficient, $h t c$, is defined as:

$$
h t c=\frac{k_{\text {fluid }} N u}{d_{h}}
$$

where $N u$ is the Nusselt number, $d_{h}$ the hydraulic diameter and $k_{\text {fluid }}$ the thermal conductivity of the fluid. 
The heat transfer model calculates the Nusselt number, $N u$, depending on the Reynolds number, $R e$. For laminar flows $(R e<2300)$, the model uses a constant Nusselt number $N u=4.089$, and for turbulent flows $(R e>2300)$, the Gnielinski semi-empirical correlation (Gnielinski, 1976):

$$
N u=\frac{\frac{f_{c}}{8}(R e-1000) \operatorname{Pr}}{1+12.7\left(\operatorname{Pr}^{2 / 3}-1\right) \sqrt{\frac{f_{c}}{8}}}
$$

where $\operatorname{Pr}$ is the Prandtl number and $f_{c}$ the Moody friction factor defined as:

$$
f_{c}=\left(\frac{1}{1.8 \log _{10} R e-1.5}\right)^{2}
$$

Gnielinski correlation is used following the model proposed by Dostal (2004). This correlation assumes that fluid properties are constant across the tube. This occurs when a fluid is heated or cooled at temperatures far away from the discontinuity line. In this case, fluid properties will be similar despite the temperature differences across the tube. However, when the fluid works close to the discontinuity line, the different temperatures across the tube may convey parts with different thermophysical properties resulting in specific heat transfer behaviours (Jackson, 1997; Pioro and Mokry, 2011).

For example, when a fluid with temperature below the discontinuity line (i.e. in the liquid region) is heated, some layers near the heating surface may reach temperatures above the discontinuity line (i.e. in the gas region). The density difference will move the gaseous volume from the heating surface to the inside of the tube. This phenomena, called boiling when the pressure is below the critical pressure and pseudo-boiling when it is above (Pioro, 2016), has specific features that Gnielinski correlation cannot predict.

Figure 4.8 shows an example of the heat transfer coefficient, htc, obtained in two experimental cases from Bae, Kim and Kang (2010) and the prediction of Gnielinski correlation. The experiments consist in $\mathrm{CO}_{2}$ flowing upwards in a vertical uniformly-heated tube at $8.12 \mathrm{MPa}$. The difference between these experiments is the heat flux. As Gnielinski correlation does not consider the heat flux, the prediction is the same for both cases. The results from the correlation agrees with the experimental values for temperatures above $50{ }^{\circ} \mathrm{C}$. The thermophysical properties of the fluid across the tube are similar at these temperatures and therefore Gnielinski correlation can predict the heat transfer coefficient. 
The correlation has more difficulties to predict the heat transfer coefficient at the beginning of heating. In the case of the lowest heat flux, the temperatures (and so the thermophysical properties) are similar across the tube. Therefore, Gnielinski correlation can predict the heat transfer behavior. However, in the case of the greatest heat flux, the gaseous layer that appears in the pseudo-boiling obstructs the heat exchange. The resulting buoyancy provokes a deterioration of the heat transfer. Gnielinski overestimates the heat transfer coefficient when there is deteriorated heat transfer. The property variation across the tube is greater as the heat flux increases and, therefore, the prediction of the correlation is even worst.

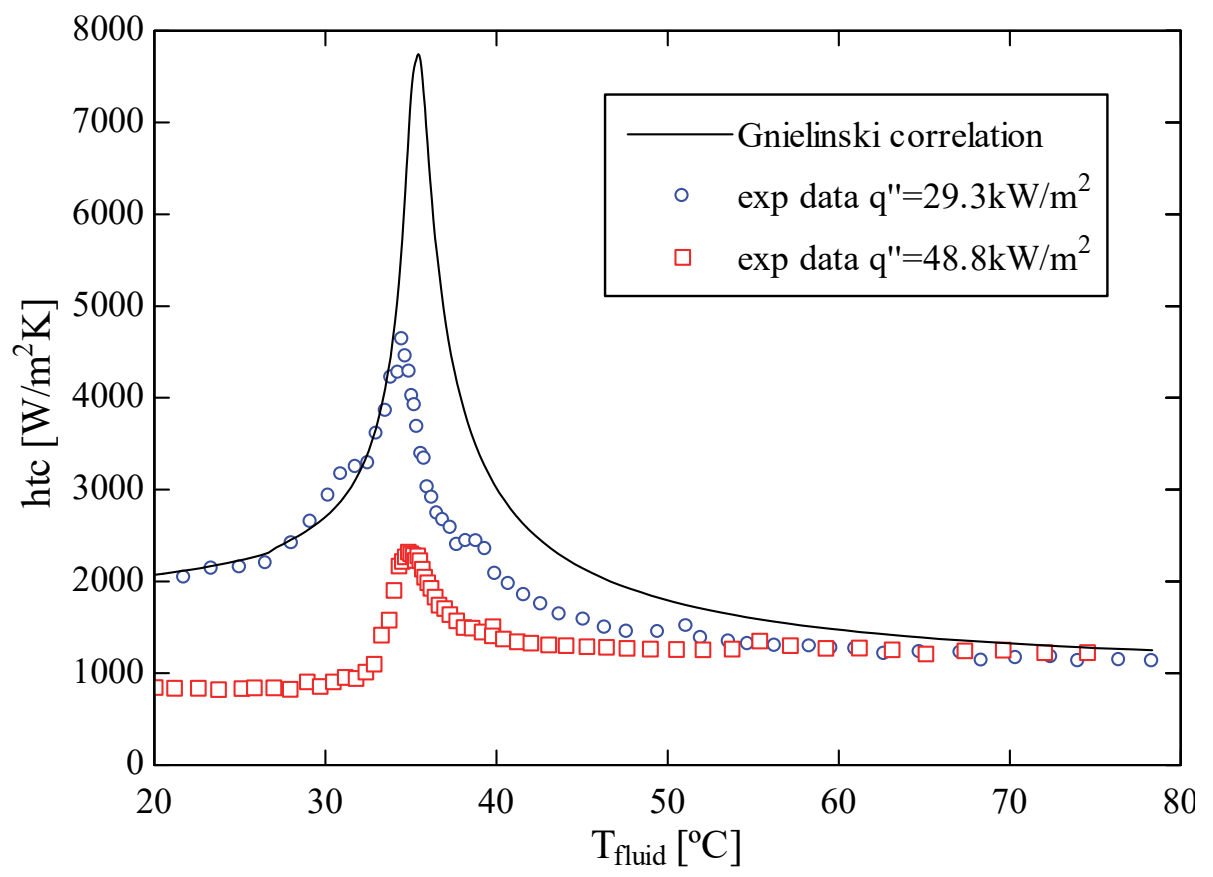

Figure 4.8. Heat transfer coefficient, $h t c$, as a function of the bulk fluid temperature, $T_{\text {fluid }}$

The magnitude of the heat transfer deterioration depends on several factors, such as heat flux, mass flux rate and orientation of the flow with respect to the gravitational field (Lei et al., 2017). Figure 4.8 represents an upward heated flow. The effect of the buoyancy may produce deteriorated heat transfer in upward heated and downward cooled flows. However, buoyancy always enhances turbulence in downward heated and upward cooled flows, which leads to high values of the heat transfer coefficient avoiding deteriorated heat transfer. Therefore, Gnielinski correlation will give better results in downward heated and upward cooled flows.

If the flow were orientated in a path with high potential of heat transfer deterioration (upward heated and downward cooled flows), then the Gnielinski correlation could be overestimating the heat transfer coefficient. In this case, other correlations should be used. However, the 
existing correlations for predicting heat transfer deterioration are case dependent (Cheng and Liu, 2017), and therefore parametric analysis at different conditions would be very tedious.

For the cases analysed in this chapter, the only heat exchanger with possibilities to experience heat transfer deterioration is the precooler. The fluid may cross the discontinuity line in this heat exchanger at some conditions. As the Gnielinski correlation is used, it can be assumed that the volume obtained is the volume of a heat exchanger with the fluid orientated to avoid deteriorated heat transfer, i.e., the $\mathrm{CO}_{2}$ flowing upwards. When the precooler crosses the discontinuity line, the high-side pressure crosses it too. However, the high-side pressure considered in this chapter is far above the critical pressure, where the property variation along this isobar is small enough to avoid heat transfer deterioration.

\subsection{Heat exchanger size in pericritical cycles with $\mathrm{CO}_{2}$}

\subsubsection{Effectiveness vs Conductance}

The exergy efficiency of regenerative cycles increases with the size of intermediate heat exchangers, which has been represented by means of the effectiveness in previous chapters. Figure 4.9 shows the exergy efficiency, $\eta_{e x}$, of pericritical $\mathrm{CO}_{2}$ cycles at conditions from Table 4.1 as a function of the IHX effectiveness, $\varepsilon$. Dry- and wet-cooled cycles are represented by dashed and continues lines, respectively, and simple and multi-heating cycles are in black and red, respectively. The low-side pressure has been optimized to maximize the exergy efficiency in each cycle. The resulting pressure ratios are also shown in Figure 4.9.

Multi-heating cycles obtain higher efficiencies than simple regenerative cycles and wetcooled cycles higher efficiencies than dry-cooled cycles. Note that, while in previous chapters the Carnot factor $\left(\tau=T_{t, i n} / T_{c, i n}\right)$ was set to a constant value $\tau=3$, in this analysis wet- and drycooled cycles have different Carnot factor, $\tau=3.21$ and $\tau=3.01$, respectively. Therefore, wetcooled cycles achieve higher efficiencies than dry-cooling.

Simple regenerative cycles work in the gaseous region in the whole range of effectiveness values. The pressure ratio of the dry-cooled cycle decreases uniformly with the effectiveness until $\varepsilon=1$. However, the pressure ratio of wet-cooled cycles is reaching a threshold at this effectiveness value. This means that the low-pressure side is close to the discontinuity line, but still below it. 

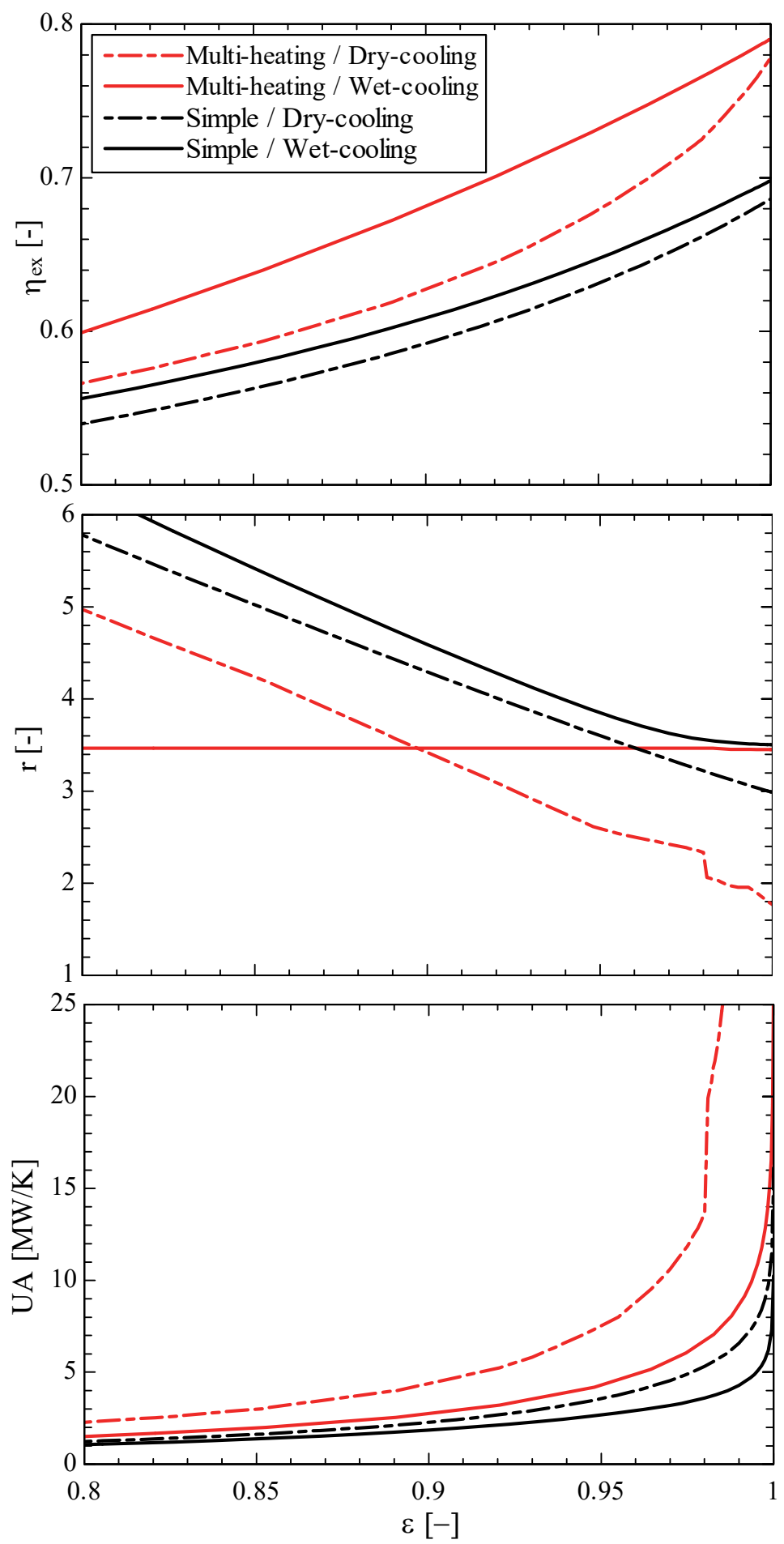

Figure 4.9. Exergy efficiency, $\eta_{e x}$, pressure ratio, $r$, and conductance, $U A$, as a function of the

IHX effectiveness, $\varepsilon$, in wet- and dry-cooled cycles with one heat supply (simple) and two heat supplies (multi-heating)

When an additional heat is supplied to both dry- and wet-cooled cycles, the pressure ratio decreases. The pressure ratio of the multi-heating dry-cooled cycle decreases uniformly with the effectiveness until $\varepsilon=0.95$. From this value on, the pressure ratio seems to be reaching a threshold, but it experiences a sharp drop at $\varepsilon=0.98$. The pressure ratios of the apparent 
threshold correspond to the gas-like region, and the pressure ratios at effectiveness above $\varepsilon=0.98$ correspond to the liquid-like region. In the case of multi-heating wet-cooled cycles, all the pressure ratios shown in Figure 4.9 correspond to the liquid region or close to it.

The exergy efficiency in simple wet-cooled cycles is higher than in simple dry-cooled cycles at the expense of larger pressure ratios, but the effect of the effectiveness in the pressure ratio and the efficiency is very similar in both cases. The exergy efficiency is also higher in multiheating wet-cooled cycles than in multi-heating dry-cooled cycles. However, the effect of the effectiveness in pressure ratio and efficiency is different in these two cases.

Under wet-cooled conditions, the efficiency difference between multi-heating and simple cycles increases with the effectiveness. However, in dry-cooled cycles, this difference is almost constant for effectiveness values below $\varepsilon=0.95$, i.e., when the low-side pressure is far away from the discontinuity line. For values above $\varepsilon=0.95$, the difference in efficiency between dry-cooled multi-heating and simple cycles starts to increase rapidly. This different behavior between dry- and wet-cooled cycles involves that, at effectiveness below $\varepsilon=0.95$, the efficiency increment of supplying an additional heat is much higher in wet-cooled cycles; but for higher effectiveness values, the efficiency increment in dry-cooled cycles increases and, in the limit of $\varepsilon=1$, the efficiency increment of supplying an additional heat in dry- and wet-cooled cycles is almost the same.

The exergy efficiency of regenerative cycles increases with the use of bigger intermediate heat exchangers whatever the definition of the size is. However, increasing the effectiveness is different from increasing the conductance, and so is its effect. A small variation of effectiveness may imply a big change in the conductance. Figure 4.9 shows the conductance, $U A$, corresponding to the IHX defined by the effectiveness, $\varepsilon$. It should be noted that the conductance increases smoothly in all the cases except in the dry-cooled cycle with multiheating when the effectiveness is $\varepsilon=0.98$. In this case, the cycle needs an abrupt change of pressure ratio to maximize the exergy efficiency, and this involves an abrupt change of conductance too.

For low effectiveness values, the conductance values barely change with the effectiveness. However, when the effectiveness gets closer to the limit $\varepsilon=1$, small effectiveness variations involve large changes in the conductance. In the limit $\varepsilon=1$, the conductance tends to infinity because the IHX would need an infinite area. The relation between effectiveness and conductance is very relevant in the relation between IHX size and exergy efficiency. For low effectiveness values, the exergy efficiency increases at the expense of small conductance 
increments. However, when the effectiveness approaches to one, larger increments of conductance are needed to increase the exergy efficiency.

The conductance values of dry-cooled cycles are greater than those of wet-cooled cycles. Moreover, for the same increment of effectiveness, dry-cooled cycles require a bigger conductance increase than wet-cooled cycles. For example, when the effectiveness goes from $\varepsilon=0.9$ to $\varepsilon=0.99$, the relative increase of conductance is $255 \%$ in the simple dry-cooled cycle, and $88 \%$ in the simple wet-cooled cycle. This means that the dry-cooled cycles will need larger increments of conductance to increase the efficiency.

Conductance values of multi-heating cycles are bigger than those of simple cycles. The multiheating cycle efficiency increases at the expense of increasing the IHX size. Dry-cooled cycles needs bigger increments of conductance to make use of multi-heating, and despite this, wet-cooled cycles achieve higher efficiencies.

It is difficult to compare the proposed cycles performance on an equivalent basis due to the large differences in conductance, especially at high effectiveness values. To analyse the relationship between cycle efficiency and conductance, all the cycles will be simulated using the conductance method to model the intermediate heat exchangers for a range of conductance values.

Note that the results obtained with the conductance and effectiveness methods may be different. For example, the maximum efficiency of a cycle with an effectiveness value defined as an input is achieved at a specific pressure ratio. This configuration results in a heat exchanger with a specific conductance. However, if the conductance were the input, the maximum efficiency could be achieved at another pressure ratio, which would imply a different effectiveness.

The difference between effectiveness and conductance methods will be especially important in multi-heating cycles. The extra variables additional-heat temperature and LTR fraction (in the conductance method) or HTR effectiveness (in the effectiveness method) makes the cycle configuration more versatile, involving bigger potential differences between both methods. An example of multi-heating cycle calculated with the conductance method will help to understand these differences. Figure 4.10 shows the influence of the LTR fraction, $x_{L T R}$, in the exergy efficiency of multi-heating wet-cooled cycles with different global conductance values, $U A$. Pressure ratio and additional-heat temperature are calculated to maximize the exergy efficiency by means of the conductance method. The resulting pressure ratio $r=3.46$ is the same in all the cases. 


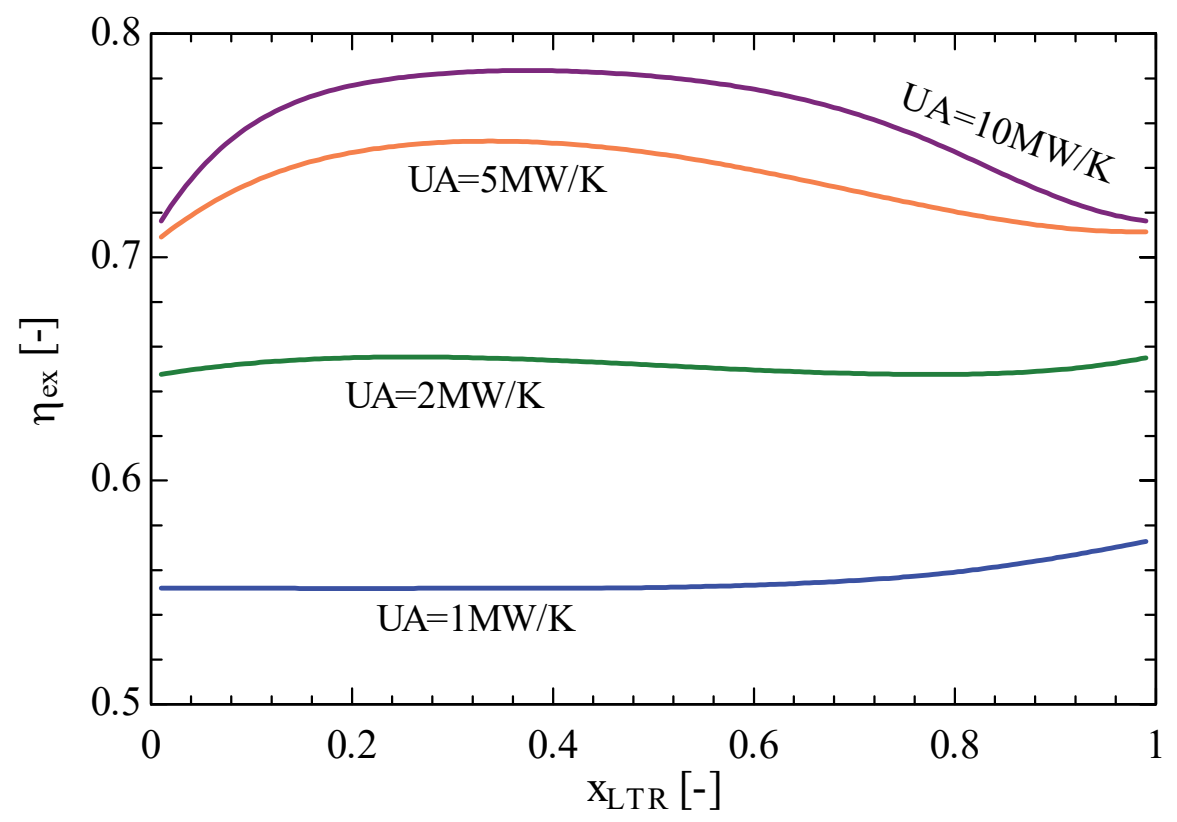

Figure 4.10. Exergy efficiency, $\eta_{e x}$, of multi-heating wet-cooled cycle with different global conductance values, $U A$, as a function of the LTR fraction, $x_{L T R}$

For the lowest conductance value, $U A=1 \mathrm{MW} / \mathrm{K}$, the maximum efficiency is obtained when the LTR fraction is $x_{L T R}=1$. This means that the benefit of multi-heating comes from the heat supply at two different temperatures, i.e., the cycle is a simple cycle with the primary heat supplied at two temperatures. When the global conductance is increased to $U A=2 \mathrm{MW} / \mathrm{K}$, the configuration with $x_{L T R}=1$ also obtain higher efficiencies than the rest of configurations. Nonetheless, the configuration with $x_{L T R}=0.3$ obtains similar efficiencies. In this configuration, the benefit of multi-heating comes not only from the lower exergetic cost that a lower temperature involves, but also from the decrease of regeneration irreversibilities. For larger conductance values, the benefit of lowering regeneration irreversibilities is larger, and the optimum configuration is between $x_{L T R}=0.3$ and $x_{L T R}=0.4$.

In order to understand the differences between effectiveness and conductance methods, it is interesting to analyse the effectiveness values corresponding to the configurations from Figure 4.10. Figure 4.11 shows HTR, LTR and global effectiveness in the cases with $U A=1 \mathrm{MW} / \mathrm{K}$ and $U A=5 \mathrm{MW} / \mathrm{K}$. The global effectiveness is almost constant in comparison to the HTR and LTR effectiveness because it represents the global HX size, in a similar way to the global conductance. Regenerators with big LTR fractions have higher LTR effectiveness and lower HTR effectiveness, and the opposite occurs when the LTR fraction is small. 


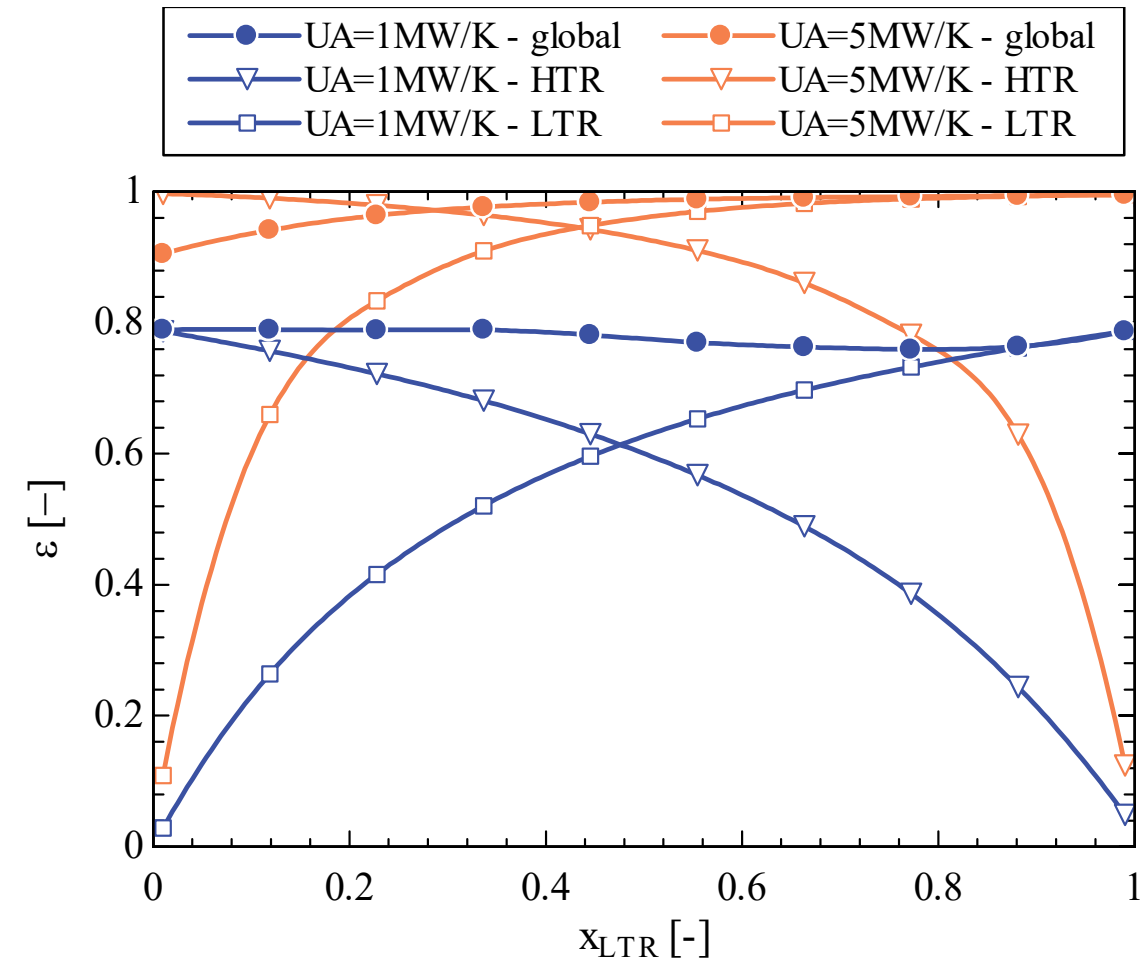

Figure 4.11. Heat exchangers effectiveness, $\varepsilon$, of multi-heating wet-cooled cycles with different global conductance values, $U A$, as a function of the LTR fraction, $x_{L T R}$

It is interesting to analyse the value of effectiveness at the point of maximum exergy efficiency. When the conductance is $U A=5 \mathrm{MW} / \mathrm{K}$, the maximum exergy efficiency is obtained at $x_{L T R}=0.33$. The values of global and HTR effectiveness at this LTR fraction are similar, i.e., the effectiveness values defined in the effectiveness method are similar. This means that the maximum exergy efficiency obtained with the effectiveness method will also be similar. However, in the case of $U A=1 \mathrm{MW} / \mathrm{K}$, the effectiveness method will give different results because HTR and global effectiveness are very different in Figure 4.11. Conductance and effectiveness methods will obtain different optimum cycle configurations (and so different efficiencies) at low conductance values.

To compare the results obtained with the effectiveness and conductance methods, all the cycles are simulated using both methods to model the intermediate heat exchangers. Pressure ratio and additional-heat temperature (in case of multi-heating) are optimized to maximize the exergy efficiency. The resulting exergy efficiency is compared as a function of the conductance value, which is an input in the conductance method and an output in the effectiveness method. Figure 4.12 shows the exergy efficiency of simple and multi-heating cycles in the cases of dry- and wet-cooling. Continuous lines represent the results obtained with the conductance method, $U A$, and dashed lines the results obtained with the effectiveness method, $\varepsilon$. Figure 4.12 also shows the pressure ratio, $r$, corresponding to each cycle. 

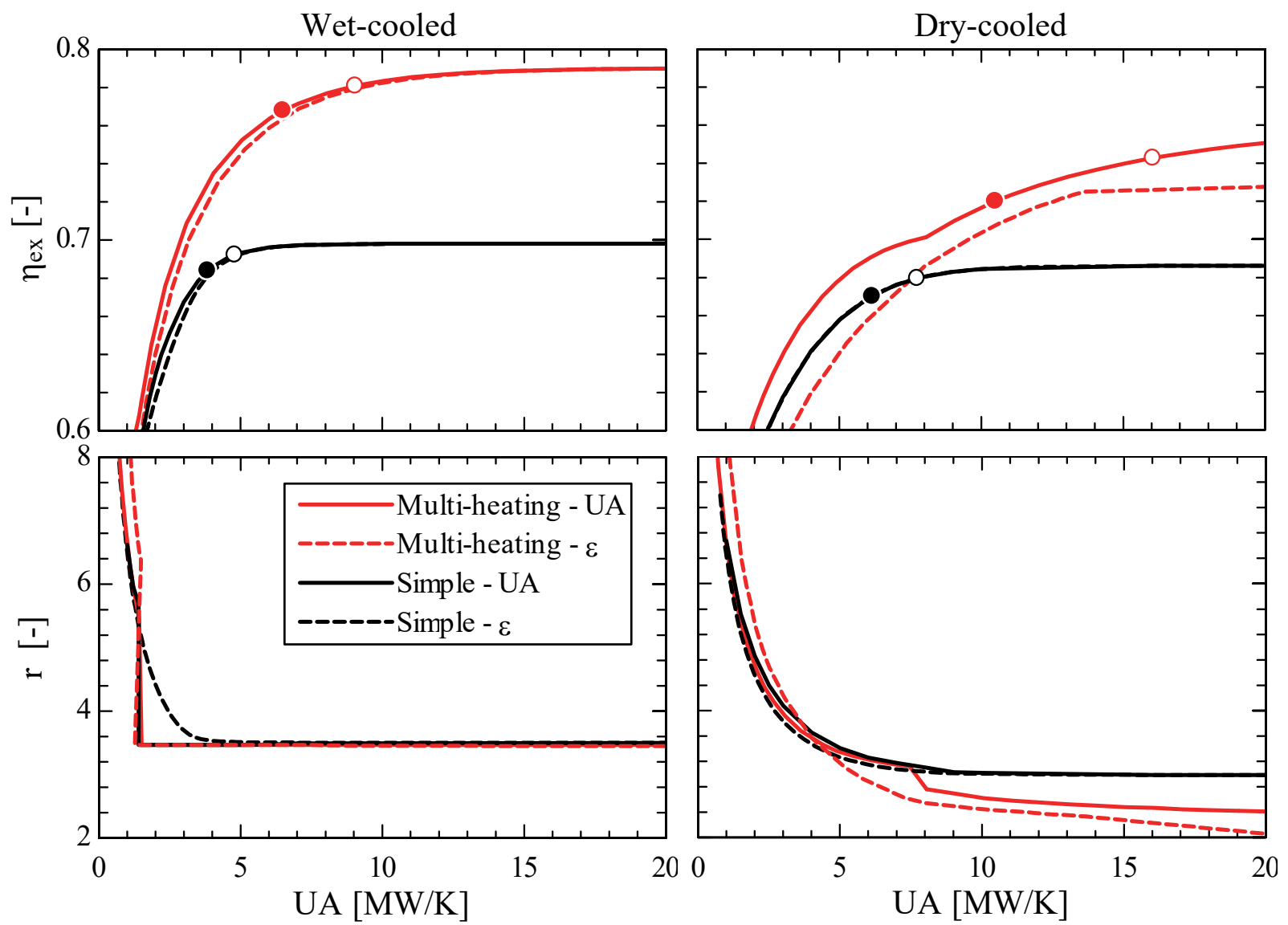

Figure 4.12. Exergy efficiency, $\eta_{e x}$, and pressure ratio, $r$, as a function of the global conductance, $U A$. Wet-cooled cycles to the left and dry-cooled cycles to the right.

The effect of the conductance in the exergy efficiency is the same in all the cases: the efficiency grows rapidly at low conductance values and slowly at high values. Horizontal asymptotes mark the end of the efficiency growth. The simple regenerative cycle asymptotes address its highest efficiency at lower conductance values than the multi-heating cycle. Thus, although multi-heating cycles achieve higher efficiencies, they need larger conductance values in order to take full advantage of its potential.

Wet-cooled cycles have higher exergy efficiencies than dry-cooled cycles due the greater Carnot factor of the former. Moreover, the asymptotes in wet-cooled cycles address its highest efficiency at lower conductance values. Wet-cooled cycles may achieve higher efficiencies with smaller IHXs. The difference of IHX sizes between wet- and dry-cooled cycles is especially large when there is multi-heating.

In all the cases of Figure 4.12, the conductance method obtains exergy efficiencies equal or greater than those calculated by means of the effectiveness method. This was expected as the results are being compared as a function of the conductance. However, the differences 
between the results obtained with both methods and the reasons of these differences depend on each case. The analysis of these differences will help to identify the advantages and disadvantages that each method has.

In the case of simple cycles, in black, the results of both methods are very similar. In simple dry-cooled simple cycles, the exergy efficiency is the same using effectiveness and conductance methods in spite of working at slightly different pressure ratios at some conductance values. In simple wet-cooled cycles, the results are different only in the range of conductances $U A=[1.5,4.5] \mathrm{MW} / \mathrm{K}$. In this range, the optimum pressure ratio calculated with the effectiveness method decreases smoothly, as it was seen in Figure 4.9. However, simple cycles with these conductance values can achieve slightly higher exergy efficiencies at smaller pressure ratios if the conductance method is used.

The results obtained with the effectiveness and the conductance methods in multi-heating wet-cooled cycles are also very similar. In this case, the differences in efficiency have nothing to do with the pressure ratio, but with the regenerator configuration. Figure 4.13 shows in red the effectiveness obtained with the conductance method for multi-heating cycles. Global and HTR effectiveness are different, while these two values are defined with same value in the effectiveness method. This is the reason of the efficiency difference. The higher the conductance value, the closer the global and HTR effectiveness values are, and therefore, the more similar the efficiency calculated with both methods is.
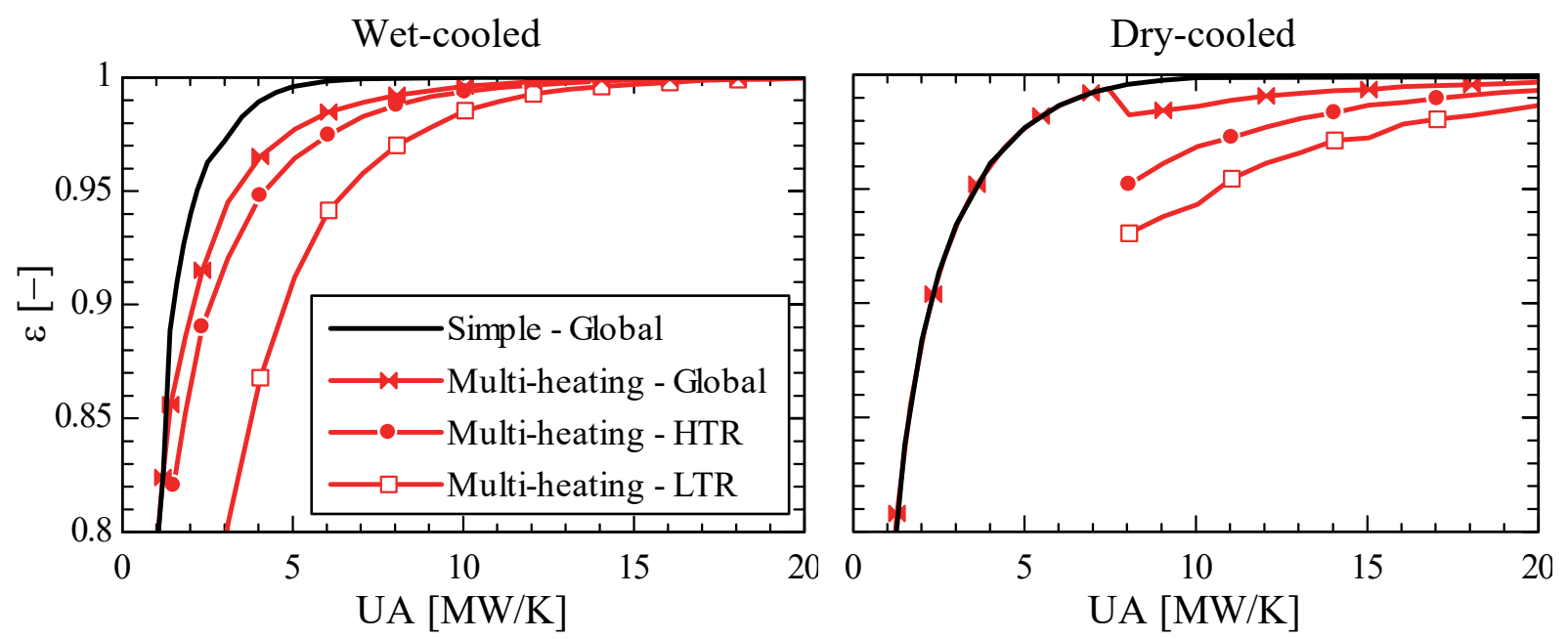

Figure 4.13. Heat exchanger effectiveness, $\varepsilon$, as a function of global conductance, $U A$, for simple and multi-heating cycles (wet-cooled to the left and dry-cooled to the right)

In dry-cooled multi-heating cycles, the differences between the effectiveness and the conductance methods are greater and have more singularities. Figure 4.12 and Figure 4.13 
show two regions in the results obtained with the conductance method separated by the conductance $U A=8 \mathrm{MW} / \mathrm{K}$. The trend of the exergy efficiency changes at this conductance value and the pressure ratio drops. The optimum cycle configuration at conductances above $U A=8 \mathrm{MW} / \mathrm{K}$ is a standard multi-heating configuration with two heat exchangers and two heats at different temperatures. However, below conductance $U A=8 \mathrm{MW} / \mathrm{K}$, the optimum configuration has only one heat exchanger, and the two heats at different temperatures are supplied in the range of enthalpies that the simple regenerative cycle does.

In dry-cooled multi-heating cycles, the exergy efficiency calculated with the effectiveness method is lower due to the different configuration in the regenerator. This difference is especially big at conductance values below $U A=8 \mathrm{MW} / \mathrm{K}$, where the conductance method considers one heat exchanger as the optimum configuration, but the effectiveness method considers the existence of a second HX in the regenerator. Moreover, the difference between both methods is also big at conductance values between $U A=14 \mathrm{MW} / \mathrm{K}$ and $U A=20 \mathrm{MW} / \mathrm{K}$. The reason is the abrupt change of conductance for a small change of effectiveness due to the discontinuity line overpass seen in Figure 4.9. When the effectiveness is $\varepsilon=0.98$, the effectiveness method jumps from $U A=14 \mathrm{MW} / \mathrm{K}$ to $U A=20 \mathrm{MW} / \mathrm{K}$ with a small change of effectiveness. The result is a straight line joining these two conductance values in Figure 4.12. The effectiveness method maximizes the exergy efficiency by reducing the pressure ratio without considering the heat exchanger size. In the case of dry-cooled multi-heating cycles, this involves an abrupt change in the conductance, and therefore big differences between the results calculated with the conductance method. However, it is observed that increasing slightly the pressure ratio in this range of effectiveness barely affects the exergy efficiency, and it decreases considerably the conductance. Thus, the results obtained with effectiveness and conductance methods could be more similar if the pressure ratio from the effectiveness method is slightly increased.

Figure 4.14 compares the results obtained with conductance and effectiveness methods in drycooled multi-heating cycles from Figure 4.12, and the results obtained with a mixed method using the effectiveness method with the pressure ratios from the conductance method, which are slightly larger. The mixed method, in green, shows more similar results to those obtained with conductance method, especially at high conductance values. This means that the effectiveness method adequately represents the cycle potential, but it may mislead the interpretation of the conductance calculation by giving too large conductance values. In this case, a simple test of different pressure ratios can give a better idea of the conductance. 


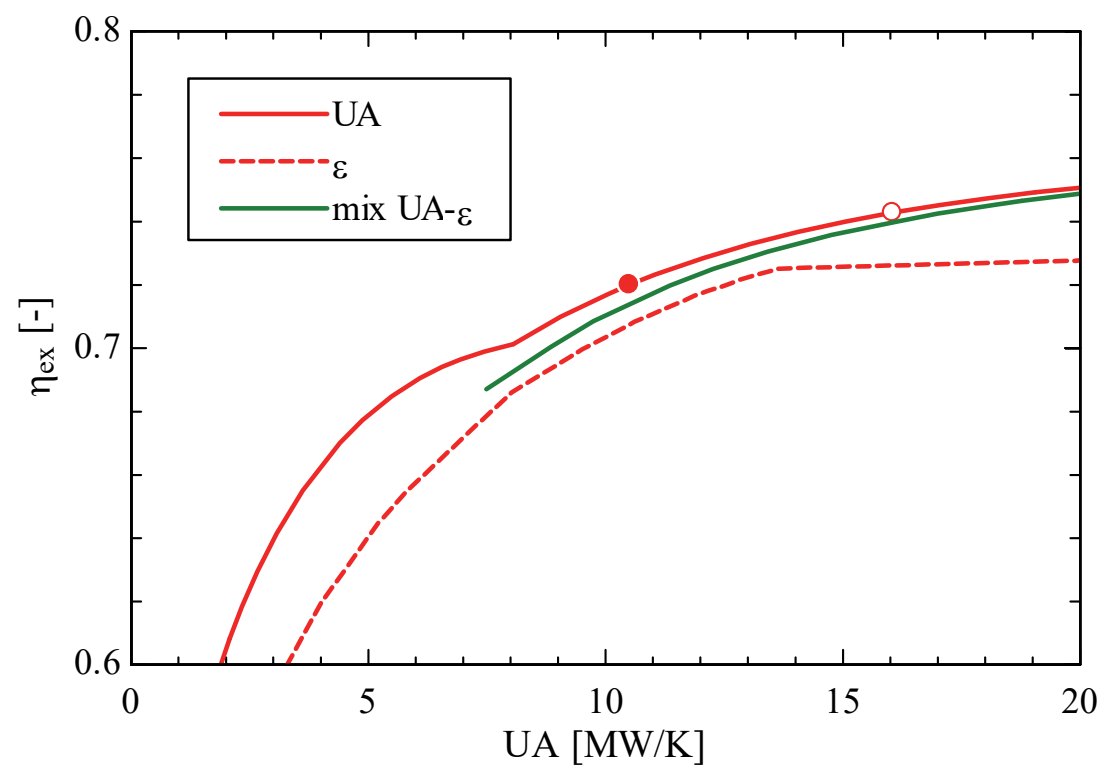

Figure 4.14. Exergy efficiency, $\eta_{e x}$, of multi-heating dry-cooled cycles as a function of the global conductance value, $U A$, obtained with three different methods: conductance, $U A$, effectiveness, $\varepsilon$, and a mix of both.

The comparison of the efficiency as a function of the regenerator conductance is very useful in pericritical $\mathrm{CO}_{2}$ cycles, in which cost is largely driven by the regenerator conductance (Neises and Turchi, 2014). Thus, the conductance method is very useful to estimate the effect of the regenerator size in the efficiency. Nevertheless, the effectiveness method should not be underestimated as it can compare the potential of cycles. All the cycles from Figure 4.12 show the threshold of exergy efficiency around a global effectiveness $\varepsilon=0.99$. Of course, the regenerator of multi-heating cycles with this effectiveness will be bigger than in simple cycles, but the exergy efficiencies will be higher too.

The conductance value corresponding to a global effectiveness $\varepsilon=0.99$ is in between the circles shown in Figure 4.12. The closed circles mark the regenerators with a minimum temperature difference of $5^{\circ} \mathrm{C}$, while the open circles indicate a minimum temperature difference of $2^{\circ} \mathrm{C}$. These temperatures may seem low for typical heat exchangers such as shell and tubes. However, they are common values in PCHEs (see specifications sheet of a PCHE in Appendix C). Despite the increase of the regenerator size to get to the point with $\varepsilon=0.99$, the increase of efficiency is large enough to consider these configurations. In fact, previous investigations about other type of $\mathrm{CO}_{2}$ pericritical layouts propose configurations with effectiveness values around $\varepsilon=0.99$ as potential solutions (Dostal, 2004). 


\subsubsection{Volume}

The conductance method is useful to estimate the impact of the regenerator size in the cycle efficiency. Nevertheless, it is important to analyse the volume associated to the conductance for two main reasons. The first one is that the conductance method do not consider neither heat transfer coefficients nor pressure drops, which could improve or worsen the results of some configurations with respect to others. The second reason is that the volume gives a more understandable idea about the real size of heat exchangers than the conductance, and the conversion from volume (or weight) to cost is easier than the conversion from conductance to cost.

The configurations obtained with the conductance method will be used to calculate the volume of the PCHEs. Each volume will be the minimum possible associated to each conductance. Figure 4.15 shows with continuous lines the regenerator volumes corresponding to wet- and dry-cooled cycles, respectively, as a function of the conductance values. Black continuous lines correspond to regenerators of simple cycles and red continuous lines to regenerators of multi-heating cycles.
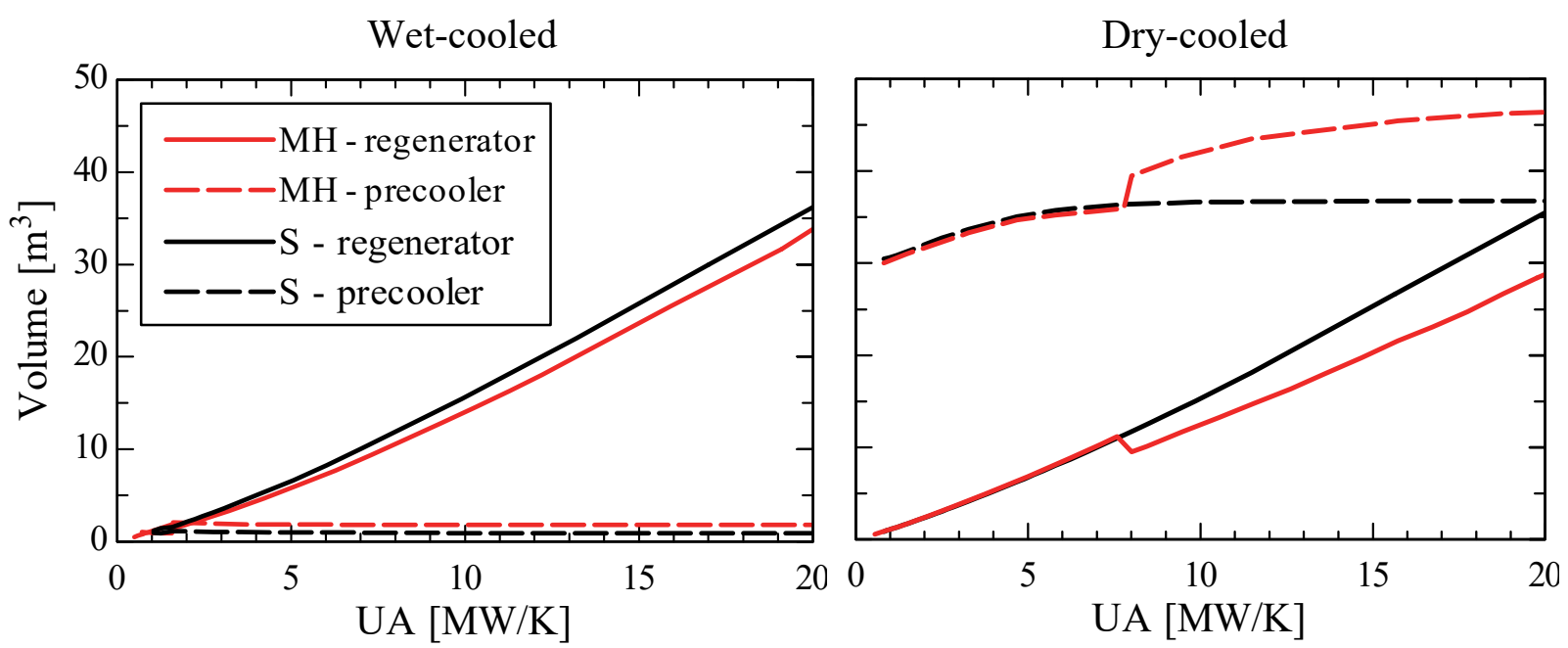

Figure 4.15. Volume of regenerator and precooler as a function of the global regenerator conductance, $U A$, in simple ( $\mathrm{S}$ ) and multi-heating $(\mathrm{MH})$ cycles in the cases of wet-cooling and dry-cooling.

The regenerator in multi-heating cycles with two heat exchangers (all the cases except multiheating dry-cooled cycles with conductance values below $\mathrm{UA}=8 \mathrm{MW} / \mathrm{K}$ ) are smaller than in simple cycles due to the PCHE design. The same pressure drop limit is set for each PCHE. Thus, the pressure drop in multi-heating cycles is double than in simple cycles, and this results in lower volumes of the multi-heating regenerator. In the case of multi-heating dry- 
cooled cycles with conductances below $\mathrm{UA}=8 \mathrm{MW} / \mathrm{K}$, as they have a single heat exchanger, the volumes are the same than in the simple cycle. This involves an abrupt change in the regenerator volume that separates the dry-cooled multi-heating cycles with one and with two heat exchangers at $\mathrm{UA}=8 \mathrm{MW} / \mathrm{K}$.

Except for the mentioned abrupt change, the regenerator volume of all cycles varies approximately linearly with the conductance. Thus, the efficiency shape as a function of the regenerator volume will be similar to the one shown in Figure 4.12 as a function of conductance.

Figure 4.15 also shows an estimation of the precooler size using PCHEs with the pressure limitations from Table 4.3. The precooler represents a small portion of the total heat exchanger size in wet-cooled cycles, while the opposite happens in dry-cooled cycles. In the case of $\mathrm{CO}_{2}$-water PCHEs, the minimum volume is obtained when the pressure drop in both sides of the PCHE is the maximum allowed. However, in the case of $\mathrm{CO}_{2}$-air PCHEs, the minimum volume is obtained when the pressure drop in the air side is the maximum allowed, but the pressure drop in the $\mathrm{CO}_{2}$ side is negligible. This means, that the air side is the limiting factor.

The precooler conductance is much smaller than the regenerator conductance in wet- and drycooled cycles. However, the small global heat transfer coefficient $U$ between air and $\mathrm{CO}_{2}$ involves huge transfer areas in the precooler of dry-cooled cycles. Other configurations of PCHEs could be used to improve the heat transfer in the air-cooler and so reduce its volume. However, previous investigations in this matter show that even with other configurations the air-cooled PCHE volume is about 100 times the size of a water-cooled PCHE (Moisseytsev and Sienicki, 2014). Several investigations propose the use of finned-tube air coolers for this type of applications (Ho et al., 2015; Neises and Turchi, 2014). A preliminary analysis of finned-tube compact heat exchangers taken from the EES library show that the volumes obtained with these heat exchangers are similar to those obtained with PCHEs. However, finned-tube compact heat exchangers are expected to be cheaper than PCHEs. Therefore, these heat exchangers seem to be a better option than PCHEs, although still representing an important part of the system.

The big volumes of the precooler in dry-cooled cycles involve large transfer areas and therefore low heat fluxes. In these conditions, the problem of heat transfer deterioration will not appear. However, the heat flux is much greater in the precooler of wet-cooled cycles, which may involve heat transfer deterioration. In this case, the models used could be 
overestimating the heat transfer coefficient and, therefore, underestimating the precooler volume. The heat transfer deterioration could be avoided with layouts with the $\mathrm{CO}_{2}$ flowing upwards. Anyway, the precooler volume would still represent a small part of the total heat exchanger volume in wet-cooled cycles.

The volume of the regenerator as a function of the conductance is similar in all the cycles. Nonetheless, the impact of the regenerator in the total heat exchanger size will be lower in dry-cooled cycles due to the larger size of the precooler. Figure 4.16 shows the exergy efficiency without considering the pumping power of all the studied cycles as a function of the total PCHE volume. The volumes needed in dry-cooled cycles are much bigger than in wet-cooled cycles. If the main cost of the cycle were represented by the PCHE volume, it might seem logical to choose wet-cooling instead of dry-cooling. However, the cost of wetcooling will also depend on the water availability. For example, a nuclear power plant with water wells close to it will better fit with wet-cooled cycles. However, in a solar thermal power plant located in a desert region, carrying water to the plant could be too expensive, and integrating it with a dry-cooled cycle could be a cheaper solution.

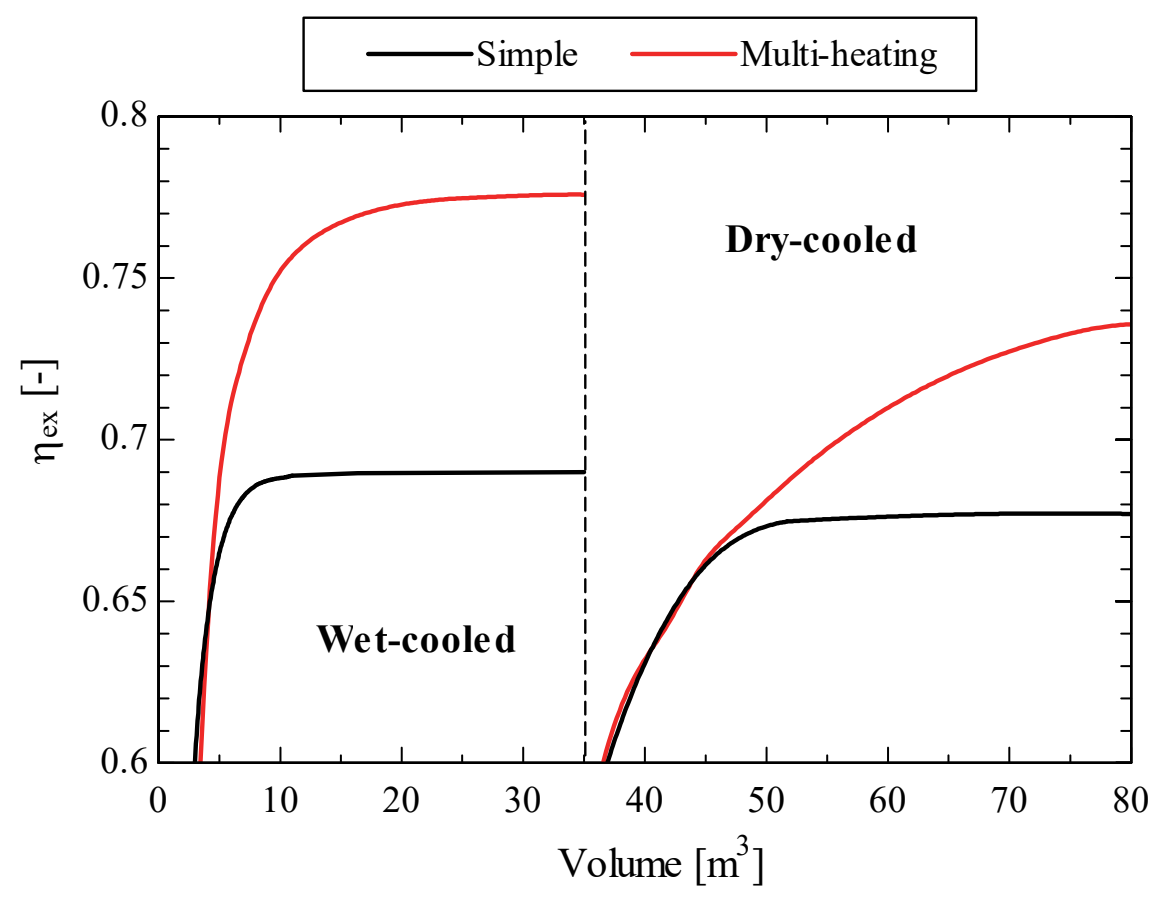

Figure 4.16. Exergy efficiency, $\eta_{e x}$, of simple and multi-heating cycles as a function of the total heat exchangers volume. The dashed line separates wet-cooled (to the left) and drycooled (to right) cycles.

Despite the bigger increments of regenerator size needed to increase the exergy efficiency in dry-cooled cycles, the relative increment with respect to the total heat exchanger volume will 
be smaller. For example, the total heat exchanger volume of wet-cooled simple cycles needs to be multiplied by three (from $3 \mathrm{~m}^{3}$ to $9 \mathrm{~m}^{3}$ ) to raise the efficiency from 0.6 to the threshold, while the volume of the dry-cooled cycle has to be increased a $40 \%$ (from $36 \mathrm{~m}^{3}$ to $50 \mathrm{~m}^{3}$ ). Therefore, if the simple dry-cooled cycle were more profitable than the simple wet-cooled cycle for a specific case, multi-heating probably could also be more profitable in the drycooled case as the relative increase in volume is smaller.

The total heat exchangers volume in wet-cooled cycles may seem very small. According to the main manufacture company of PCHEs, Heatric (www.heatric.com), the volume of PHCEs may be up to $85 \%$ smaller than traditional technologies such as shell and tube heat exchangers. Moreover, previous investigations show similar results. Dostal (2004) analyzed simple and recompression cycles, the latter with very similar characteristics in the regeneration to multi-heating cycles. The total volume obtained to reach the thermal efficiency threshold is around $0.2 \mathrm{~m}^{3} / \mathrm{MW}_{\mathrm{e}}$ and $0.4 \mathrm{~m}^{3} / \mathrm{MW}_{\mathrm{e}}$ in wet-cooled simple and recompression cycles, respectively. In Figure 4.16, these thresholds are reached at $0.15 \mathrm{~m}^{3} / \mathrm{MW}_{\mathrm{e}}$ and $0.3 \mathrm{~m}^{3} / \mathrm{MW}_{\mathrm{e}}$. The small discrepancies with Dostal's results are mainly due to the small differences in some of the cycle parameters, such as the compressor inlet temperature.

Dostal (2004) stated that heat exchangers in pericritical cycles with $\mathrm{CO}_{2}$ are the biggest components. In order to show the difference between the size of heat exchangers and turbomachine, the rotor diameters of the turbomachines are calculated for some of the cycles presented in this chapter. The software developed by Dyreby (2014) is used for this purpose. Table 4.4 shows the results. Despite the small size of heat exchangers, the turbomachines are even smaller. The greatest difference occurs in dry-cooled multi-heating cycles, which means that the design of heat exchangers becomes especially important in these cases.

\subsection{Summary}

The main purpose of this chapter has been to analyze the regeneration phase of pericritical cycles, both simple and multi-heating cycles. The exergy efficiency of these cycles is compared for different methods of defining the intermediate heat exchangers: effectiveness, conductance and volume. The impact of the regeneration in the exergy efficiency is evaluated for two compressor inlet temperatures, which depend on the type of cooling used: dry- and wet-cooling. 
Table 4.4. Size of the components depending on the cycle type

\begin{tabular}{lcccc}
\hline \multirow{2}{*}{ Parameter } & \multicolumn{2}{c}{ Wet-cooling } & \multicolumn{2}{c}{ Dry-cooling } \\
\hline Regenerator volume & Simple & Multi-heating & Simple & Multi-heating \\
Precooler volume & $5.1 \mathrm{~m}^{3}$ & $9.4 \mathrm{~m}^{3}$ & $9.6 \mathrm{~m}^{3}$ & $16.3 \mathrm{~m}^{3}$ \\
Compressor rotor diameter & $1 \mathrm{~m}^{3}$ & $1.8 \mathrm{~m}^{3}$ & $36 \mathrm{~m}^{3}$ & $44.1 \mathrm{~m}^{3}$ \\
Turbine rotor diameter & $0.28 \mathrm{~m}$ & $0.28 \mathrm{~m}$ & $0.45 \mathrm{~m}$ & $0.4 \mathrm{~m}$ \\
Exergy efficiency & $0.55 \mathrm{~m}$ & $0.55 \mathrm{~m}$ & $0.61 \mathrm{~m}$ & $0.65 \mathrm{~m}$ \\
\hline
\end{tabular}

The exergy efficiency raises when the intermediate heat exchanger size increases. However, the method of defining the size affects the optimum configuration of the cycle, and therefore its performance. The effectiveness method is very useful to understand the potential of the cycle. However, the optimum cycle configuration with high effectiveness may involve small optimum pressure ratios, and therefore too big heat exchangers. Thus, it is important to evaluate the thermal conductance and the volume of the intermediate heat exchangers.

The type of heat exchanger used to calculate the heat exchanger volume is the Printed Circuit Heat Exchanger (PCHE). The exergy efficiency grows when the heat exchanger size increases either if this size is defined by its conductance or by its volume. This growth is fast at small sizes and slows down at big sizes, tending to a horizontal asymptote. The transition between these two types of growths is marked by an effectiveness $\varepsilon=0.99$. Simple regenerative cycle asymptotes address its highest efficiency at smaller sizes than the multi-heating cycle. Thus, although multi-heating cycles achieve higher efficiencies, they need bigger intermediate heat exchangers in order to take full advantage of its potential. Wet-cooled cycles may achieve higher efficiencies with smaller intermediate heat exchangers than dry-cooled cycles. The difference of intermediate heat exchangers size between wet- and dry-cooled cycles is especially large when there is multi-heating.

This chapter also estimates the volume of the precooler in order to compare it with the volume of intermediate heat exchangers. The precooler size depends on the cooling method, it represents a small part of the total heat exchangers size in wet-cooled cycles, and a big part of dry-cooled cycles. The estimated total size of heat exchangers needed in optimum multiheating cycles is around $0.4 \mathrm{~m}^{3} / \mathrm{MW}_{\mathrm{e}}$ when there is wet-cooling and $1.6 \mathrm{~m}^{3} / \mathrm{MW}_{\mathrm{e}}$ when there 
is dry-cooling. The smaller size of heat exchangers in wet-cooling cycles comes along with slightly higher pressure ratios, but also higher exergy efficiencies. Wet-cooled cycles seem to have more advantages, but the lack of water could involve a preference for dry-cooled cycles. 


\section{MulTi-HEATING CYCLES IN CONCENTRATING SOLAR POWER PLANTS}

Multi-heating cycles show a substantial increase in efficiency with respect to simple regenerative cycles at the expense of bigger heat exchangers. But multi-heating cycles do not only need bigger heat exchangers, they also need to have access to heat sources at different ranges of temperature. This requirement must be satisfied according to the origins of the available heat in the power plant. This is difficult to do with combustion devices, in which the smoke (combustion products) are generated with the maximum achievable temperature. However, it can be perfectly done in Concentrating Solar Power (CSP) plants, in which the heat transfer fluid can carry the heat within a selected range of temperatures, according to solar conditions, concentrator field and receiver features.

This chapter studies the conditions that the solar field of a CSP plant must fulfil in order to take full advantage of multi-heating cycles. A new type of solar field is created with this purpose: the multi-heating solar tower. The configuration of a multi-heating solar tower is similar to the configuration of a solar tower, but with the receiver and the heliostats divided into two sections with different requirements in concentration ratio, fluid temperature, and absorbed heat flux. The purpose of this division is to adapt the solar field to the requirements of multi-heating cycles. The new system uses the basis of thermal coherence to obtain higher efficiency with lower material requirements. 
The efficiency of the multi-heating solar tower coupled to a multi-heating cycle will be compared under different conditions with the efficiency of a standard solar tower coupled to a simple regenerative cycle, and a techno-economic analysis will help to identify the most critical components of the new system with multi-heating.

\subsection{The receiver in a Concentrating Solar Power plant}

The receiver is the element of a CSP plant in charge of absorbing the solar radiation and transferring its energy to the heat transfer fluid. The resulting thermal energy is supplied to the power block, who transforms it into electricity. This section analysis the features of a general receiver and the requirements that must fulfil to supply heat to the power cycle.

\subsubsection{Model of receiver}

The solar radiation impinges in the receiver after having been reflected by concentrators. The thermal power reaching the receiver is defined in Equation 5.1, where $A_{h e l}$ is the area of the heliostats field, DNI the Direct Normal Irradiance and $\eta_{\text {opt }}$ the optical efficiency of the concentration process.

$$
\dot{Q}_{\text {rec }}=A_{\text {hel }} \cdot D N I \cdot \eta_{\text {opt }}
$$

All this power is concentrated in the receiver area. The concentration is defined by a dimensionless parameter called geometrical concentration ratio, that relates heliostats and receiver area:

$$
C_{\text {geo }}=\frac{A_{\text {hel }}}{A_{\text {rec }}}
$$

If the concentration considers the optical efficiency, then it is called optical concentration:

$$
C_{o p t}=\eta_{o p t} C_{g e o}
$$

And Equation 5.1 can be redefined as:

$$
\dot{Q}_{\text {rec }}=A_{\text {rec }} \cdot D N I \cdot C_{\text {opt }}
$$

Part of the radiation reaching the receiver is absorbed by the heat transfer fluid (HTF) and the rest is lost. Equation 5.5 represents an energy balance to the receiver of a solar thermal power plant. 


$$
\alpha \cdot \dot{Q}_{r e c}=\dot{Q}_{H T F}+\dot{Q}_{c v}+\dot{Q}_{r a d}
$$

The left term represents the thermal power absorbed by the receiver, where $\alpha$ is the receiver absorptivity, and the right term is the sum of the thermal power transmitted to the HTF, $\dot{Q}_{H T F}$, and the convection and radiation losses, $\dot{Q}_{c v}$ and $\dot{Q}_{r a d}$, respectively.

The thermal powers belonging to the right term are defined in Equations 5.6-5.8.

$$
\begin{gathered}
\dot{Q}_{H T F}=A_{r e c} \cdot U \cdot\left(T_{r e c}-T_{H T F}\right) \\
\dot{Q}_{c v}=A_{r e c} \cdot h_{\text {loss }} \cdot\left(T_{r e c}-T_{a i r}\right) \\
\dot{Q}_{r a d}=A_{r e c} \cdot F \cdot \varepsilon_{r} \cdot \sigma \cdot\left(T_{r e c}{ }^{4}-T_{s k y}{ }^{4}\right)
\end{gathered}
$$

The receiver surface is considered to be at the same temperature, $T_{r e c}$, in internal and external surfaces, i.e., in the surface in contact with the HTF and in the surface in contact with the environment. The heat transfer coefficient from receiver surface to HTF, $U$, determines the capacity of the receiver to transfer heat to the HTF with a temperature $T_{H T F}$. Moreover, the receiver also exchanges heat with the environment. This heat exchange is by convection with the air and by radiation with the sky. The heat transfer coefficient from receiver surface to air, $h_{l o s s}$, defines convection losses and view factor, $F$, and receiver emissivity, $\varepsilon_{r}$, define radiation losses. Note that the radiation losses are simplified considering that the heat exchange is only against the sky. This is a conservative assumption as other surfaces such as the ground will be at higher temperatures, which would involve lower radiation heat losses.

The sky temperature, $T_{s k y}$, will be calculated as a function of the air temperature (Swinbank, 1963):

$$
T_{\text {sky }}=0.0552 \cdot T_{\text {air }}^{1.5}(K)
$$

Table 5.1 shows the input parameters used in the analysis of the receiver. DNI and air temperature are representative values of summer in Almería (Spain) (EnergyPlus, 2018). The rest of the values are common values used in CSP simulations (Ho and Iverson, 2014; Muñoz, Martinez-Val and Ramos, 2011; Padilla et al., 2015a). The value of the heat transfer coefficient from receiver surface to $\mathrm{HTF}, U$, will depend on the fluid features and the receiver design. This study will keep it constant in order to leave its influence out of the results. The maximum possible view factor, $F$, is set in order to consider the worst-case scenario. The value of this factor will mainly depend on the receiver design. 
Table 5.1. Input parameters used for the analysis of a solar thermal receiver

Parameter

Direct Normal Irradiance, $D N I$

Air temperature, $T_{\text {air }}$

Emissivity, $\varepsilon r$

Absorptivity, $\alpha$

View factor, $F$

Heat transfer coefficient from receiver surface to air, $h_{\text {loss }}$

Heat transfer coefficient from receiver surface to fluid, $U$
Value

$900 \mathrm{~W} / \mathrm{m}^{2}$

$40{ }^{\circ} \mathrm{C}$

$0.15-0.85$

0.95

1

$10 \mathrm{~W} / \mathrm{m}^{2} \mathrm{~K}$

$2000 \mathrm{~W} / \mathrm{m}^{2} \mathrm{~K}$

Emissivity and absorptivity properties from Table 5.1 are given by the receiver coating. The coating commonly used in solar towers is Pyromark ${ }^{\circledR}$. This coating has a high solar absorptance $(\alpha \sim 0.95)$, but also high emittance $(\varepsilon r \sim 0.87)$. Other coating such as cermet coatings (currently used in linear receivers) have better optical properties (much lower emittance). However, they are sensitive to oxidation and suffer performance degradation at temperatures greater than $500{ }^{\circ} \mathrm{C}$, and therefore they are not well-suited for solar tower applications, where higher temperatures are reached. This is the reason why while the receiver emissivity in solar tower models is commonly set to around 0.85 (Ho and Iverson, 2014; Padilla et al., 2015a), much lower values (0.1-0.2) are used in linear receivers (Forristall, 2003; Montes et al., 2009; Muñoz, Martinez-Val and Ramos, 2011).

The relation of all the parameters from Table 5.1 will determine the amount of thermal power that the HTF absorbs in relation to the total power impinging the receiver, which is the definition of receiver efficiency, $\eta_{\text {rec: }}$ :

$$
\eta_{r e c}=\frac{\dot{Q}_{H T F}}{\dot{Q}_{r e c}}
$$

The receiver efficiency is a function of the optical concentration ratio, $C_{o p t}$, and fluid temperature, $T_{H T F}$. Figure 5.1 shows the efficiency of receivers with fluids at temperature $T_{H T F}$ and solar radiation impinging in the receiver at optical concentration ratio $C_{\text {opt }}$. For the moment it is assumed that the fluid temperature is constant in the whole receiver. The receiver efficiency grows rapidly at low concentration ratios and asymptotes at high concentration 
ratios. Increasing the concentration ratio beyond the limit where the efficiency growth is almost null would lead to more complex and expensive systems with no efficiency gain. This limit depends on the fluid temperature (and therefore on the receiver surface temperature). Fluids at low temperature address this threshold at lower concentration ratios.

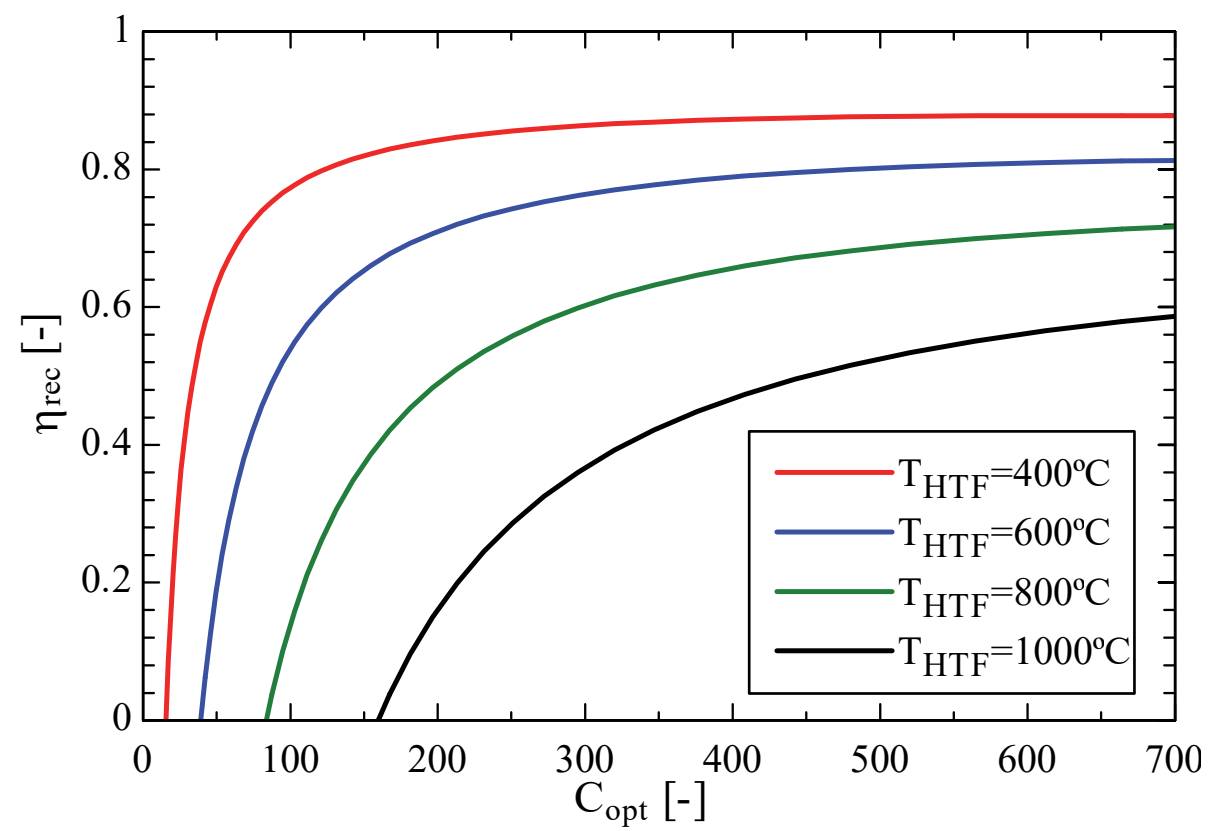

Figure 5.1. Receiver efficiency, $\eta_{r e c}$, as a function of the optical concentration ratio, $C_{o p t}$, for different HTF temperatures, $T_{H T F}$

A receiver with low temperature can achieve higher efficiencies. However, the power cycle could reach higher efficiencies if the receiver supplied heat at higher temperatures. The efficiency of a system composed of a receiver and a power cycle will be a mixture of both efficiencies. The system efficiency of a cycle with Carnot efficiency, $\eta_{C}$, coupled to a receiver with efficiency $\eta_{r e c}$ is defined in Equation 5.11.

$$
\eta_{\text {syst }}=\eta_{C} \eta_{\text {rec }}
$$

Figure 5.2 shows the efficiency of systems with different concentration ratios $\left(C_{\text {opt }}=50\right.$, $C_{o p t}=200$ and $\left.C_{o p t}=600\right)$ as a function of the turbine inlet temperature, $T_{t, i n}$. Carnot and receiver efficiencies of the systems are also represented. The Carnot efficiency, $\eta_{c}$, belongs to a cycle with cooling temperature $T_{\text {cold }}=30^{\circ} \mathrm{C}$ and turbine inlet temperature, $T_{t, i n}$, and receiver efficiency, $\eta_{r e c}$, to a receiver with HTF temperature $T_{t, i n}$. It is assumed that HTF and turbine inlet temperatures are the same. 


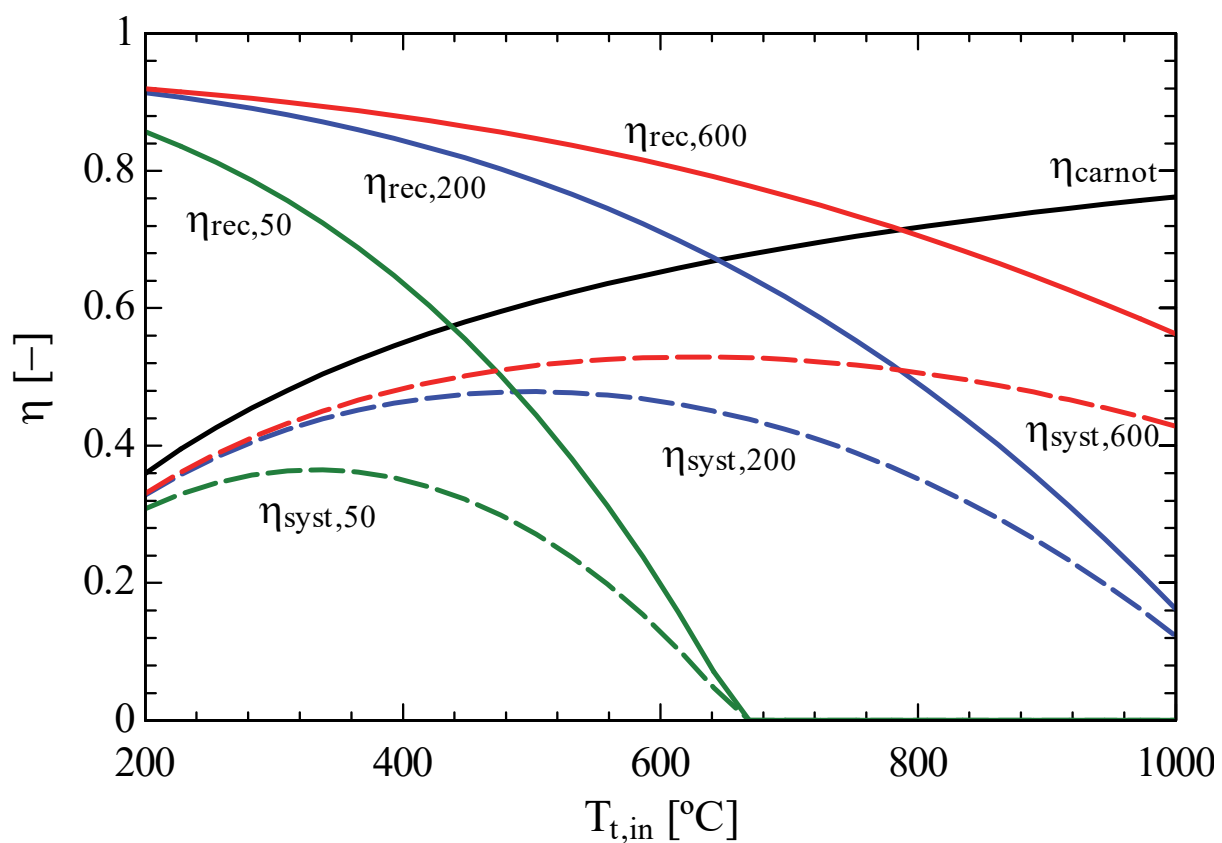

Figure 5.2. Receiver efficiency, $\eta_{r e c}$, Carnot efficiency, $\eta_{C}$, and system efficiency, $\eta_{\text {syst }}$, as a function of the turbine inlet temperature, $T_{t, i n}$, for optical concentration ratios $C_{\text {opt }}=50$,

$$
C_{\text {opt }}=200 \text { and } C_{\text {opt }}=600
$$

The receiver efficiency decreases with the turbine inlet temperature and, on the contrary, the Carnot efficiency increases. The multiplication of both efficiencies involves that there is a temperature with maximum system efficiency. This maximum depends on the concentration ratio. The decrease of receiver efficiency is more pronounced at low concentration ratios. Thus, the maximum system efficiency at low concentration ratios occurs at lower turbine inlet temperatures. On the contrary, high concentrations are needed in order to integrate the receiver with high-temperature power cycles. The optimum configuration of a CSP plant must carefully integrate the variables concentration and temperature.

\subsubsection{Benchmark against System Advisor Model}

The differences in receiver efficiency are directly related to the receiver areas needed to absorb the solar radiation. Figure 5.3 shows the receiver area needed to transmit $\dot{Q}_{H T F}=140 \mathrm{MW}_{\mathrm{t}}$ to the fluid as a function of the optical concentration for different fluid temperatures. This thermal power is the one needed to generate $50 \mathrm{MW}_{\mathrm{e}}$ in a common Rankine cycle with efficiency around 0.35 . Low optical concentrations need large areas to generate the same power, and these areas depend on the fluid temperature. High temperatures (desired to increase the cycle efficiency) involve high radiation losses and therefore, have lower receiver efficiencies and larger receiver areas. In fact, not all the temperatures can be 
obtained with all the optical concentrations. For example, an optical concentration $C_{\text {opt }}=120$ can be used to heat a fluid up to $400{ }^{\circ} \mathrm{C}$ and $600{ }^{\circ} \mathrm{C}$, but the area required to obtain $800{ }^{\circ} \mathrm{C}$ would be huge and it would be impossible to get to $1000{ }^{\circ} \mathrm{C}$.

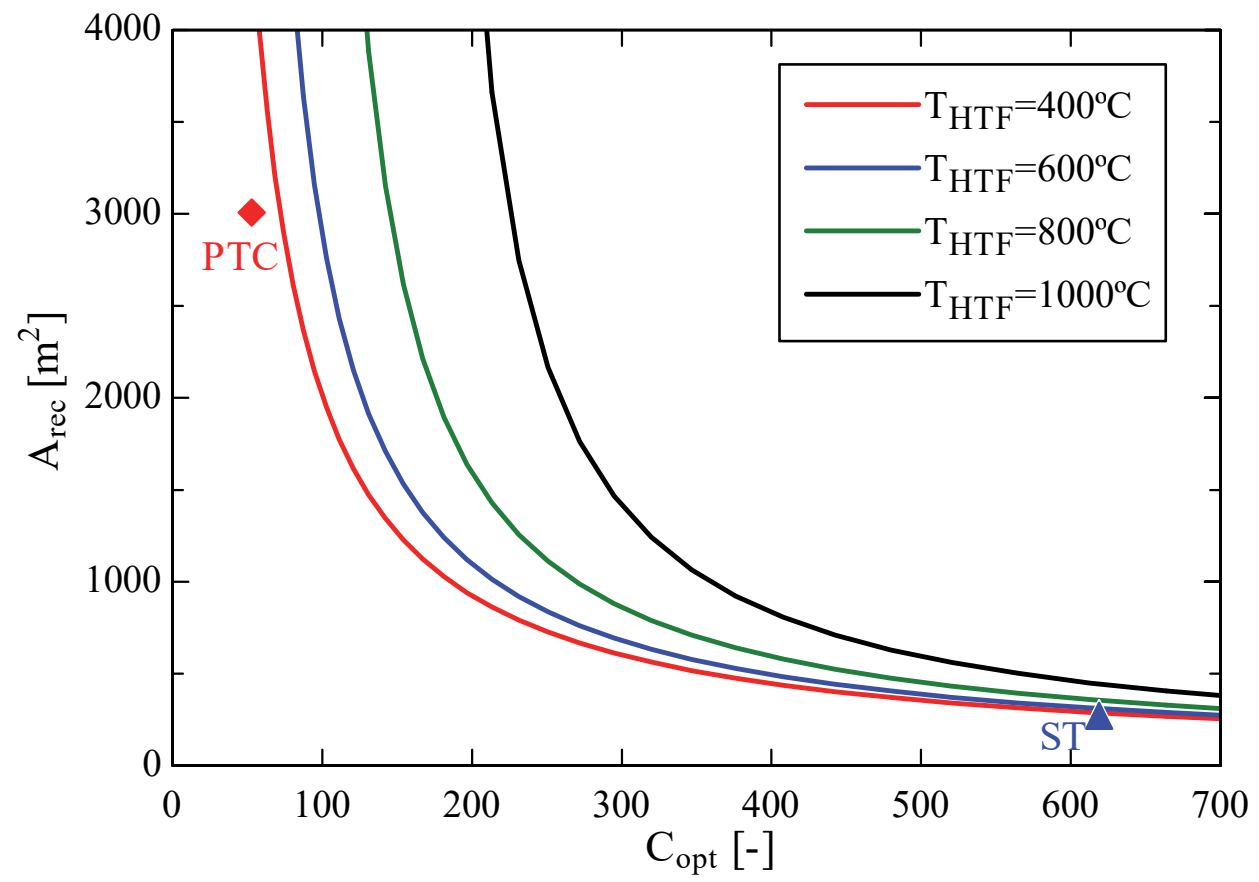

Figure 5.3. Receiver area, $A_{r e c}$, as a function of the optical concentration, $C_{\text {opt }}$, for different temperatures

The efficiency asymptote shown in Figure 5.1 can be identified in Figure 5.3. High concentrations need much smaller areas than those needed at low concentrations. A smaller area will represent, a priori, lower complexity. Moreover, pressure drops will be smaller than in the larger lengths required by small concentrations ratios. In summary, moving photons is more effective than moving heat.

Two different CSP systems, Parabolic Trough Collector (PTC) and Solar Tower (ST), are simulated with the mature software System Advisor Model (NREL, 2017) in order to show the validity of the trends obtained with the receiver model used in this chapter. These two types of plant have been selected as they are the most common ones in commercial CSP systems (Mehos et al., 2017) and they have different concentration ratios. The CSP plant in System Advisor Model (SAM) has been optimized for Almería weather data (EnergyPlus, 2018) to generate $140 \mathrm{MW}_{\mathrm{t}}$.

The receiver areas needed in the systems with PTC and ST are shown in Figure 5.3. The maximum fluid temperatures of PTC and ST are around $400{ }^{\circ} \mathrm{C}$ and $600{ }^{\circ} \mathrm{C}$, respectively. It can be observed that the receiver model proposed in last section and the results from SAM 
agree properly. Note that the lines shown in Figure 5.3 have been calculated with emissivity $\varepsilon_{r}=0.85$, while the emissivity of real PTCs is much lower. Thus, the $400{ }^{\circ} \mathrm{C}$ line and the value obtained with SAM would be closer if the same emissivity were used. Moreover, the temperature decreases along the receiver, which means that the average temperature in a PTC is below $400{ }^{\circ} \mathrm{C}$, and therefore closer to the value obtained with SAM.

Actual commercial CSP plants integrate PTCs and STs with Rankine cycles. The purpose of this chapter is to integrate the cycles previously studied, simple and multi-heating cycles, with the most adequate receiver. The analysis of the receiver must be carried out considering the needs of the power block.

\subsubsection{Power block needs}

The thermal power that the receiver must supply is determined by the cycle conditions. The cycles in this chapter will be defined by means of the effectiveness method with an effectiveness $\varepsilon=0.99$ and the conditions from Table 4.1. The turbine inlet temperature $T_{t, \text { in }}=700{ }^{\circ} \mathrm{C}$ forces the receiver to supply heat at high temperatures. Solar Towers, capable of achieving high concentration ratios, will adapt well to these temperatures. Thus, an optical concentration ratio $C_{\text {opt }}=600$ and an emissivity $\varepsilon_{\mathrm{r}}=0.85$, characteristic of Solar Towers, will be used as starting point in the analysis of the receiver. Once the receiver performance is understood, these two parameters will be varied according to its possibilities to analyze its effect on the receiver performance. The rest of the parameters of the receiver are shown in Table 5.1 and will be maintained constant during the analysis.

Simple and multi-heating cycles will use $\mathrm{CO}_{2}$ as working fluid. Although the HTF in the receiver could also be the same $\mathrm{CO}_{2}$, it is considered that is a different fluid. Thus, a heat exchanger will be needed to transfer the thermal power from the HTF to the $\mathrm{CO}_{2}$. A difference of temperatures $\Delta T=25{ }^{\circ} \mathrm{C}$ between the two fluids will simulate the heat exchanger. Thus, the HTF must be able to withstand, at least, $725^{\circ} \mathrm{C}$. Any fluid with good storage features able to withstand these temperatures could be the HTF. Although it is out of the purpose of this work to evaluate the HTF candidates, it must be mentioned that some molten salts seem to be potential candidates for these conditions (Mehos et al., 2017).

The energy balance in a heat exchanger with constant difference of temperatures $\Delta T$ implies that:

$$
\dot{m}_{\mathrm{HTF}} c_{p, \mathrm{HTF}}=\dot{m}_{\mathrm{CO}_{2}} c_{p, \mathrm{CO}_{2}}
$$


As the mass flow rates $\dot{m}_{\mathrm{HTF}}$ and $\dot{m}_{\mathrm{CO}_{2}}$ will have a constant value along the heat exchanger, it is assumed that the specific heats $c_{p, H T F}$ and $c_{p, C_{2}}$ will also have a constant value. Although this is not strictly true, the specific heat of an HTF such as molten salt hardly changes with the temperature (Pfleger et al., 2015) and the same occurs with $\mathrm{CO}_{2}$ at high pressures in the range of temperatures considered for the heat supply.

The solar radiation heats the HTF and the HTF heats the $\mathrm{CO}_{2}$. The thermal power required by the $\mathrm{CO}_{2}$ is given by the thermodynamic cycle in a specific range of temperatures. The HTF supplies this power at a temperature $25{ }^{\circ} \mathrm{C}$ greater than the $\mathrm{CO}_{2}$ temperature. The solar thermal power will depend on the HTF temperature, i.e., the energy balance in the receiver presented in Equations 5.1-5.9 will be different depending on the HTF temperature. For example, in a simple cycle that needs to heat $\mathrm{CO}_{2}$ from $449{ }^{\circ} \mathrm{C}$ to $700{ }^{\circ} \mathrm{C}$, the $\mathrm{HTF}$ supplies the power by increasing the temperature from $474{ }^{\circ} \mathrm{C}$ to $725^{\circ} \mathrm{C}$. The energy balance will be different at $474{ }^{\circ} \mathrm{C}$ and at $725^{\circ} \mathrm{C}$, and therefore the solar thermal power needed will be different in each region. Thus, heat is divided into steps, and the energy balance is applied to each step. The calculations will divide the heat in 10 steps.

Figure 5.4 shows the temperatures along a receiver integrated with a simple cycle that requires a thermal power $\dot{Q}_{\mathrm{CO}_{2}}=106 \mathrm{MW}$ to raise the $\mathrm{CO}_{2}$ temperature from $449{ }^{\circ} \mathrm{C}$ to $700{ }^{\circ} \mathrm{C}$. The temperatures of $\mathrm{CO}_{2}, \mathrm{HTF}$ and receiver surface are represented as a function of the thermal power $\dot{Q}_{\mathrm{CO}_{2}}$ accumulated along the heating. The $\mathrm{CO}_{2}$ temperature increases along the heating, and the other two temperatures follow its tendency.

Each mark in the lines represents one step. The step temperature is an average of the temperatures in the step. Thus, the minimum temperature shown in the $\mathrm{CO}_{2}$ is above $449{ }^{\circ} \mathrm{C}$ and the maximum below $700{ }^{\circ} \mathrm{C}$. The HTF temperature is $25{ }^{\circ} \mathrm{C}$ greater than the $\mathrm{CO}_{2}$ temperature, and the surface temperature is calculated in each step with the energy balance presented in Equations 5.1-5.9.

Thermal powers $\dot{Q}$ resulting from the energy balance are shown in Figure 5.5. Each step represents a thermal power absorbed by the HTF $\dot{Q}_{H T F}=10.6 \mathrm{MW}$. Convection and especially radiation losses, $\dot{Q}_{c v}$ and $\dot{Q}_{r a d}$, increase along the heating due to the higher HTF temperatures. Thus, a larger solar thermal power impinging on the receiver, $\dot{Q}_{r e c}$, is needed. As the DNI is considered constant $\left(900 \mathrm{~W} / \mathrm{m}^{2}\right)$, a larger receiver surface is needed to increase the solar thermal power reaching the receiver. 


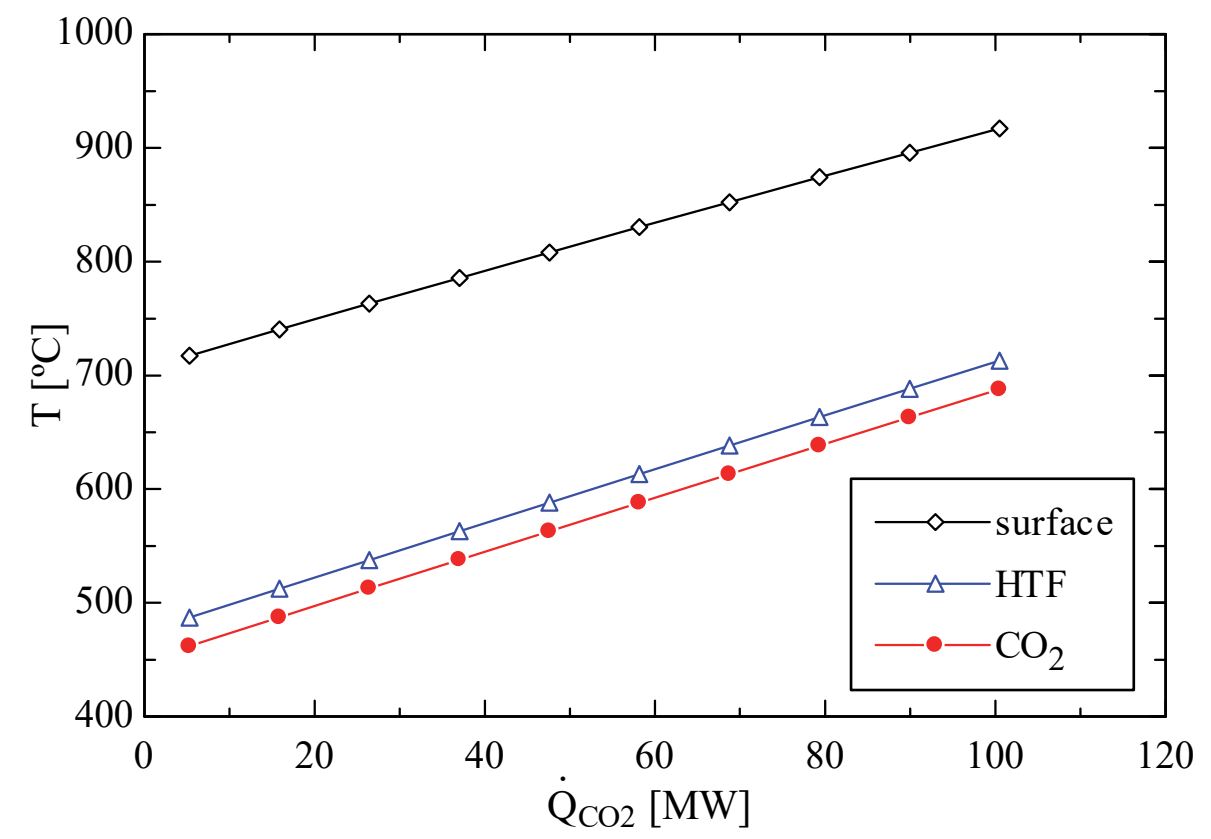

Figure 5.4. Temperatures of $\mathrm{CO}_{2}, \mathrm{HTF}$ and receiver surface as a function of the thermal power $\dot{Q}_{\mathrm{CO}_{2}}$ required by a simple cycle

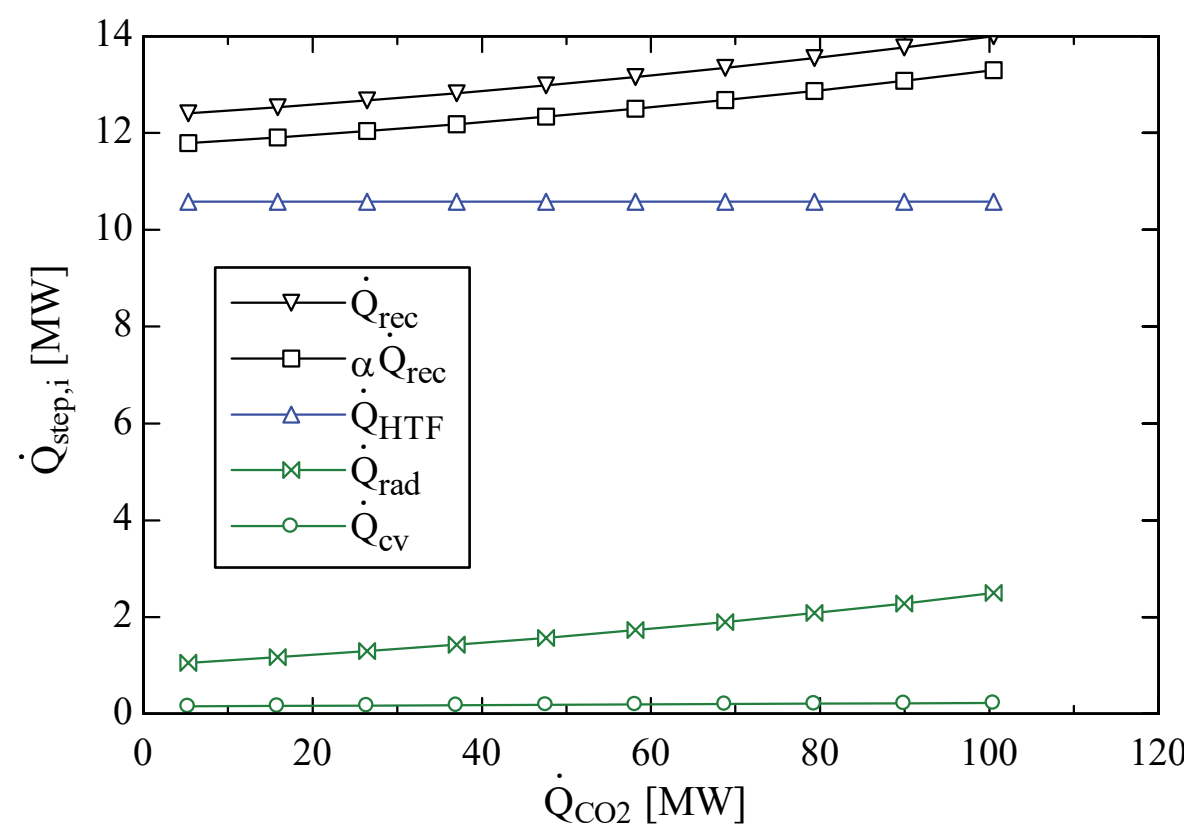

Figure 5.5. Thermal power in the receiver resulting from the energy balance in each step, $\dot{Q}_{\text {Step }_{i}}$, as a function of the thermal power $\dot{Q}_{\mathrm{CO}_{2}}$ required by a simple cycle

The thermal power required by a simple regenerative cycle is within a specific range of temperatures. Any standard receiver such as PTC or Solar Tower, designed to withstand specific conditions of solar radiation and fluid temperature, can supply this thermal power. However, multi-heating cycles need heat supply at different ranges of temperature. Thus, a 
receiver with sections at different ranges of temperature will be needed. The generic name for a receiver with these features will be multi-heating receiver.

\subsection{The Multi-Heating receiver}

A multi-heating receiver is composed of two or more sections with different thermal features. Each of these sections is a receiver itself with the efficiency defined in Equation 5.10. The efficiency of the whole multi-heating receiver integrates the efficiencies of all its sections. Equation 5.13 defines the efficiency of a multi-heating receiver with $n$ sections.

$$
\eta_{\text {rec }}=\frac{\sum_{n} \dot{Q}_{H T F, i}}{\sum_{n} \dot{Q}_{r e c, i}}=\frac{\sum_{n} \dot{Q}_{H T F, i}}{\sum_{n} \frac{\dot{Q}_{H T F, i}}{\eta_{r e c, i}}}
$$

In this chapter, the multi-heating receiver will be coupled to a multi-heating cycle with one additional heat. Thus, the multi-heating receiver will have two sections $(n=2)$. These two sections will be named primary heat and additional heat, corresponding to the high- and lowtemperature heats, respectively. Note that Equation 5.13 with $n=1$ defines the efficiency of a standard receiver with a single range of temperatures.

The high temperatures required by the cycles studied in previous chapters $\left(700{ }^{\circ} \mathrm{C}\right)$ make Solar Tower a good candidate to be integrated with pericritical $\mathrm{CO}_{2}$ cycles. In case of multiheating, the Solar Tower will be divided into two sections: primary-heat section and additional-heat section. The primary-heat section will work like in a Solar Tower, and the additional-heat section will work at lower temperature, adjusting the required solar heat flux according to the temperature. This new type of Solar Tower will be called Multi-Heating Solar Tower.

Figure 5.6 shows a scheme of solar field with Solar Tower and other with Multi-Heating Solar Tower. While in the former case all the heliostats concentrate the solar radiation into the same receiver area, the heliostats in the solar field with Multi-Heating Solar Tower will concentrate the radiation into different receiver areas.

CSP systems integrating standard solar tower and multi-heating solar tower will be compared in this chapter. The standard solar tower will be coupled to simple regenerative cycles and the multi-heating solar tower to multi-heating cycles. 


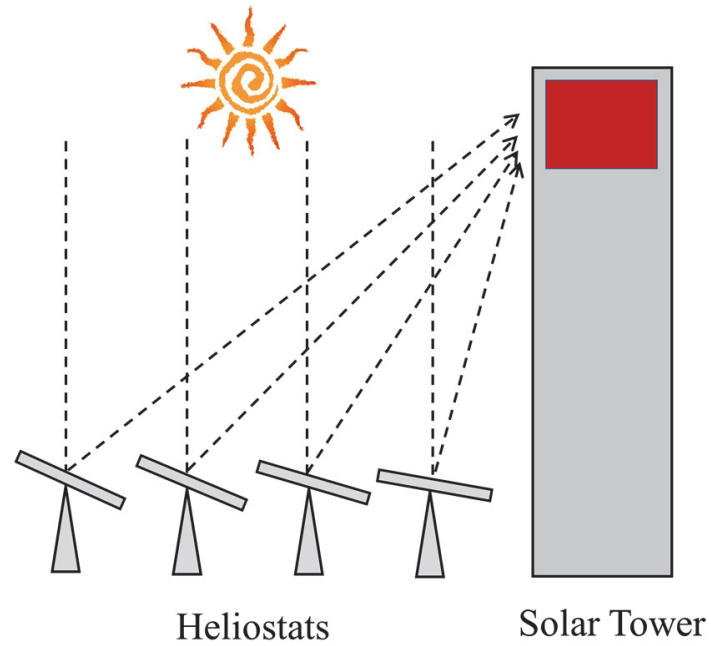

(a)

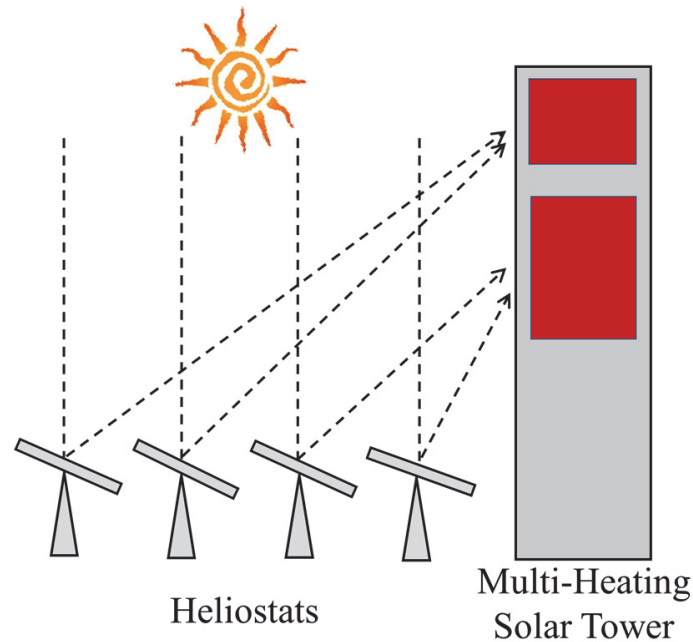

(b)

Figure 5.6. Scheme of solar field with (a) Solar Tower and (b) Multi-Heating Solar Tower

\subsubsection{Sections at different temperature}

The only features that the receiver need to know about the cycle are the thermal powers and the range of temperatures of these powers. Figure 5.7 shows an example of Multi-Heating Solar Tower coupled to a multi-heating cycle. The thermal powers $\dot{Q}$ correspond to a wetcooled multi-heating cycle with the conditions from Table 4.1, heat exchanger effectiveness $\varepsilon=0.99$, low-side pressure $P_{L}=7.2 \mathrm{MPa}$ and turbine inlet temperature $T_{\text {t,in }}=700{ }^{\circ} \mathrm{C}$. The total thermal power $\dot{Q}$ supplied by the Multi-Heating Solar Tower (MHST) is the sum of the thermal power of its two sections: primary heat ( $\mathrm{ph})$ and additional heat (ah). The thermal powers are represented as a function of the maximum additional-heat temperature in the cycle, $T_{a h}$, which means that the corresponding HTF temperature is $T_{a h}+25^{\circ} \mathrm{C}$.

The additional heat substitutes the primary heat when the additional-heat temperature increases. When the additional-heat temperature is high, the smaller thermal power required in the primary heat involves a smaller range of temperatures along this heat supply. The result is a higher average temperature and therefore a lower receiver efficiency of the primary-heat section.

The maximum primary-heat efficiency corresponds to the case with minimum additional-heat temperature, which has no additional heat (additional-heat temperature is the compressor outlet temperature). Note that this case represents a simple regenerative cycle coupled to a standard Solar Tower. 

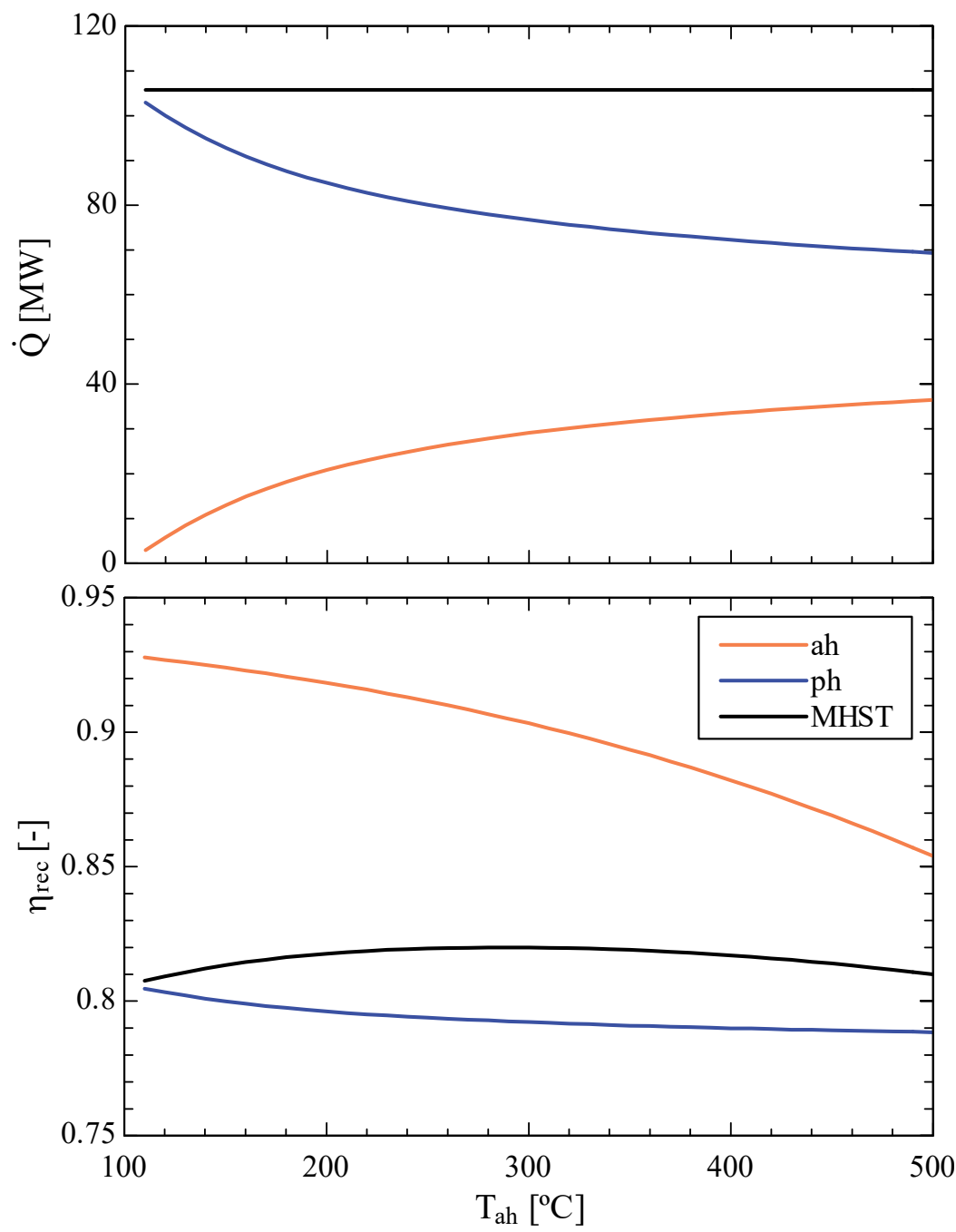

Figure 5.7. Thermal power, $\dot{Q}$, and receiver efficiency, $\eta_{r e c}$, of a Multi-Heating Solar Tower (MHST) and its two sections primary heat (ph) and additional heat (ah) as a function of the additional-heat temperature, $T_{a h}$

The receiver efficiency of the additional heat, $\eta_{\text {rec,ah }}$, also depends on the additional-heat temperature, $T_{a h}$. The additional-heat efficiency decreases with the temperature. Nonetheless, these additional heats with lower efficiency can supply a larger amount of thermal power. The result is that there is an optimum additional-heat temperature for which the maximum efficiency of the Multi-Heating Solar Tower is achieved. In Figure 5.7, this temperature is $T_{a h}=285^{\circ} \mathrm{C}$. The additional-heat power in this optimal configuration represents $26 \%$ of the total power supplied by the Multi-Heating Solar Tower.

The results shown in Figure 5.7 are obtained for a turbine inlet temperature $T_{t, i n}=700{ }^{\circ} \mathrm{C}$, i.e., for a maximum HTF temperature $T_{H T F}=725^{\circ} \mathrm{C}$. The receiver efficiency of the primary heat at this temperature is around $\eta_{r e c, p h}=0.8$. If the turbine inlet temperature varies then this 
efficiency will also vary. Figure 5.8 shows the efficiency of a multi-heating solar tower (MHST) and its two sections, primary heat (ph) and additional heat (ah) as a function of the turbine inlet temperature, $T_{t, i n}$. The optimum additional-heat temperatures have been calculated in each case. All the efficiencies decrease with the turbine inlet temperature. The efficiency of multi-heating solar tower has a value in between the efficiency of its two sections, but it is closer to the lowest efficiency one, i.e., the one at the highest temperature. This is due to the behavior of the efficiencies in Equation 5.13 as resistors in parallel.

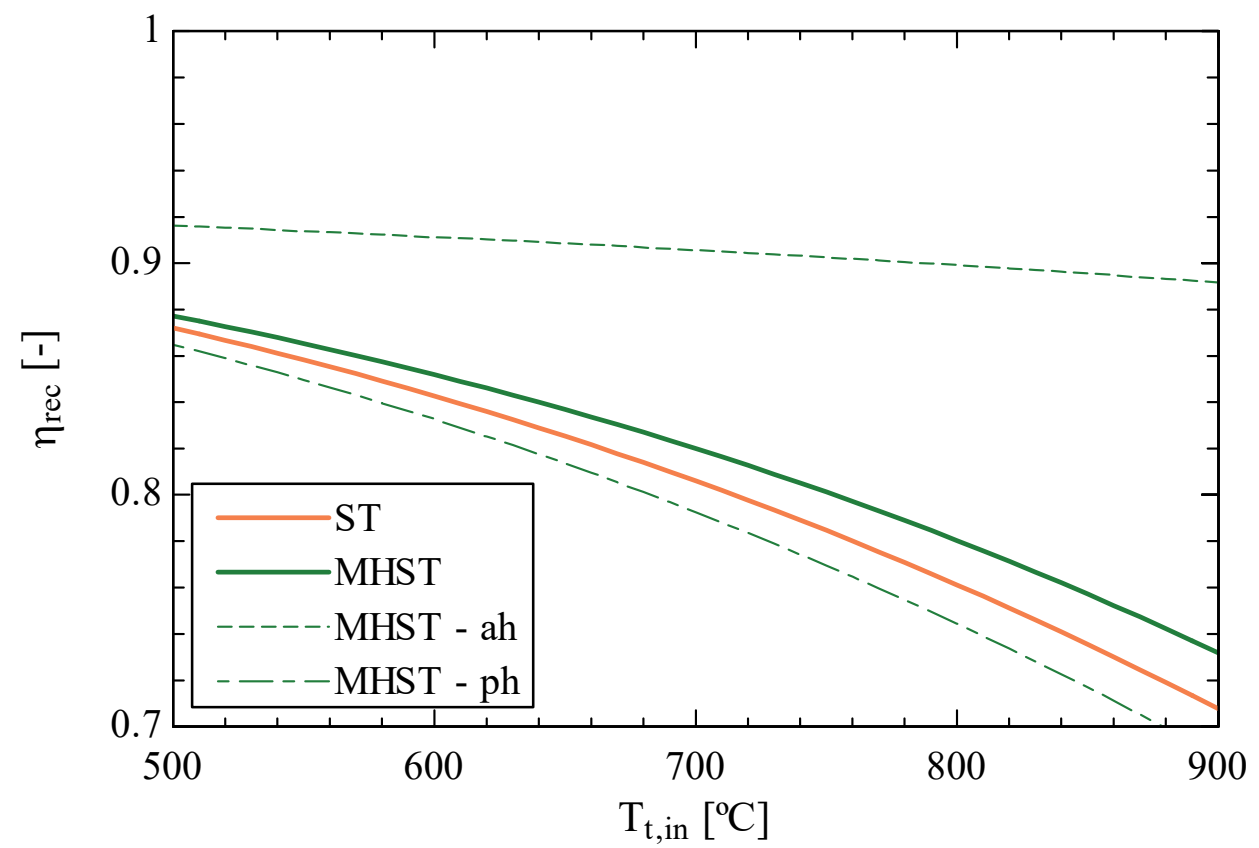

Figure 5.8. Receiver efficiency, $\eta_{r e c}$, of standard Solar Tower (ST) and Multi-Heating Solar Tower (MHST) with two sections, primary heat (ph) and additional heat (ah), as a function of the turbine inlet temperature, $T_{t, i n}$

The efficiency of a Solar Tower (ST) with the same cycle conditions than the cycle coupled to the multi-heating solar tower, but with a single heat, is also shown in Figure 5.8. Both ST and MHST efficiencies decrease with the turbine inlet temperature, but the former is higher in all the cases due to the higher efficiency of the additional heat.

\subsubsection{Sections with different concentration ratio}

The receivers shown in Figure 5.7 and Figure 5.8 are designed to receive a solar radiation with optical concentration ratio $C_{o p t}=600$, which is characteristic of Solar Towers. However, the receiver efficiency will depend on this value. Figure 5.9 shows the multi-heating solar tower efficiency and the efficiency of its two sections (to supply additional and primary heats) as a function of the concentration ratio, $C_{o p t}$. The turbine inlet temperature is $T_{t, i n}=700{ }^{\circ} \mathrm{C}$, 
and the additional-heat temperature is calculated in each case to maximize the receiver efficiency. The multi-heating solar tower efficiency has a value in between the efficiency of its two sections, but it is closer to the primary-heat section.

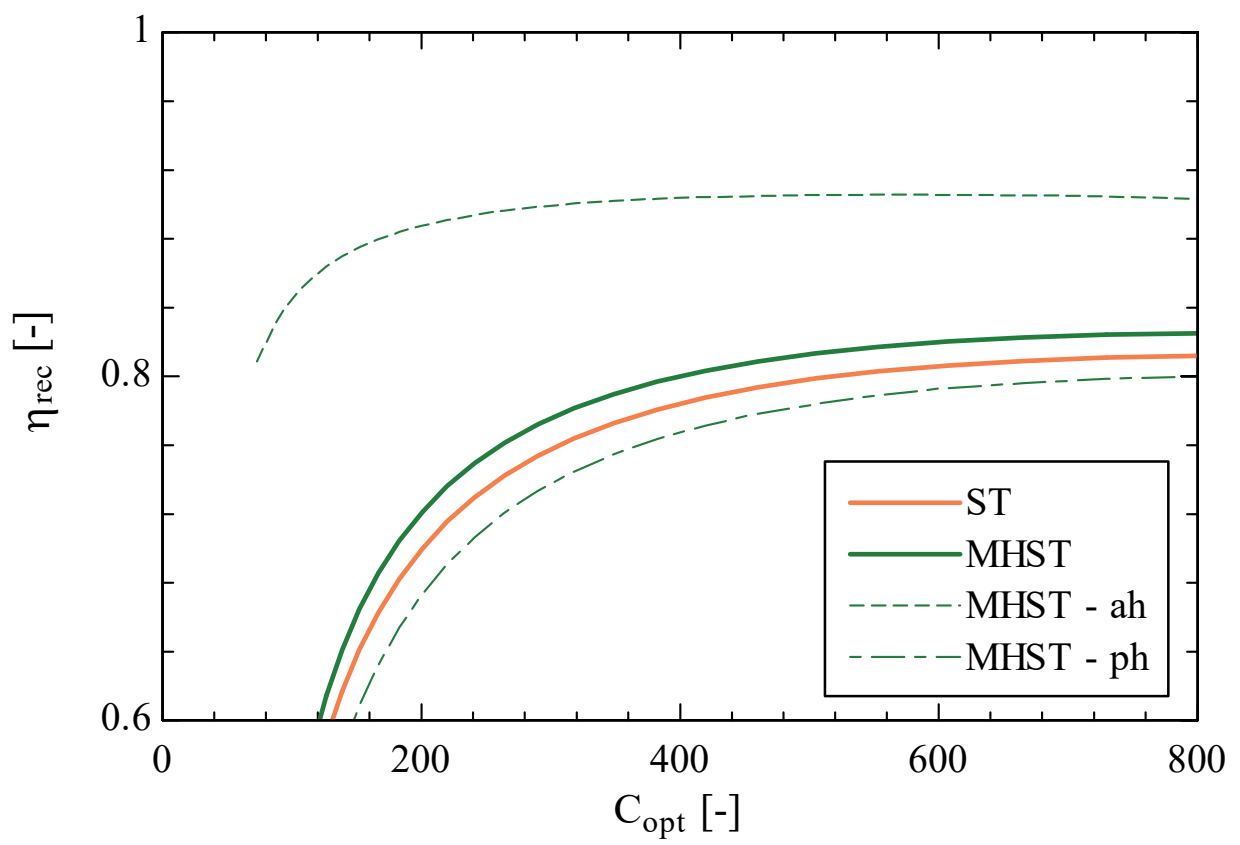

Figure 5.9. Receiver efficiency, $\eta_{r e c}$, of standard Solar Tower (ST) and Multi-Heating Solar Tower (MHST) with two sections, primary heat (ph) and additional heat (ah), as a function of the optical concentration ratio, $C_{\text {opt }}$

The efficiency of a Solar Tower is also represented in Figure 5.9 with the purpose of comparing standard Solar Tower and multi-heating solar tower. The efficiency of the multiheating solar tower is greater in all the cases. Nonetheless, the shape of the curves is similar. The receiver efficiency increases rapidly at low concentration ratios and asymptotes towards its highest efficiency at high concentration ratios.

Increasing the concentration ratio reduces the receiver area and increases the receiver efficiency at the expense, generally, of more complex and expensive concentrators. Once the efficiency asymptote is reached, the receiver area decreases slowly and the receiver efficiency barely increases. Therefore, increasing the concentration in the asymptote will involve a more expensive system without increasing the efficiency. The optimum configurations of CSP systems are expected to have concentration ratios below the asymptote, but close to it.

It will be considered that the concentration ratios belonging to the asymptote will be those fulfilling Equation 5.14. 


$$
\frac{\Delta \eta / \eta}{\Delta C_{\text {opt }} / C_{\text {opt }}}<0.05
$$

The purpose of Equation 5.14 is to analyze the starting point of the asymptote in different receivers. The value 0.05 is chosen to satisfy the condition that the concentration ratio $C_{\text {opt }}=600$ in the case of solar tower belongs to the beginning of the asymptote. Other values would result in different starting points. Nonetheless, the comparison between the different receivers would follow similar trends.

The asymptote in the multi-heating solar tower and in its primary heat begins at concentration ratios similar to those of the solar tower. These values are $C_{\text {opt }}=530$ in the case of the multiheating solar tower and $C_{o p t}=605$ in the primary-heat section. On the contrary, in the additional heat, the asymptote is reached at a much lower concentration ratio $C_{\text {opt }}=180$. Thus, this section of the multi-heating solar tower could be designed to receive a smaller solar heat flux and still maintain a high receiver efficiency.

Reducing the solar heat flux impinging on the receiver does not only involve simpler concentrators, but also softer thermal requirements in the receiver. The temperature difference between inner and outer surface of the receiver will decrease at smaller heat fluxes, which involve smaller thermal stresses (Neises, Wagner and Gray, 2014). In the model proposed in this work, inner and outer surfaces of the receiver are considered equal. However, this temperature difference can be appreciated in the fluid and surface temperatures. Figure 5.10 shows maximum fluid and surface temperatures of the two sections of a multi-heating solar tower as a function of the concentration ratio, $C_{\text {opt }}$. The multi-heating solar tower is the same than in Figure 5.9.

The temperature difference between fluid and receiver surface is greater when the concentration ratio is high, i.e., when the solar heat flux impinging on the receiver is large. For the reference concentration ratio $C_{o p t}=600$, the maximum surface temperature in the primary-heat section is $927{ }^{\circ} \mathrm{C}$ and $555{ }^{\circ} \mathrm{C}$ in the additional-heat section. These high temperatures force the receiver to use Pyromark ${ }^{\circledR}$ as coating, which is able to withstand these temperatures. However, if the surface temperatures were below $500{ }^{\circ} \mathrm{C}$ then other coatings such as cermet coatings, with better optical properties, could be employed. Figure 5.10 shows that the additional-heat section can work at surface temperatures below $500{ }^{\circ} \mathrm{C}$ if the concentration ratio is below $C_{o p t, a h}=455$. The additional-heat section could be covered by cermet coatings and, therefore, improve the receiver efficiency. 


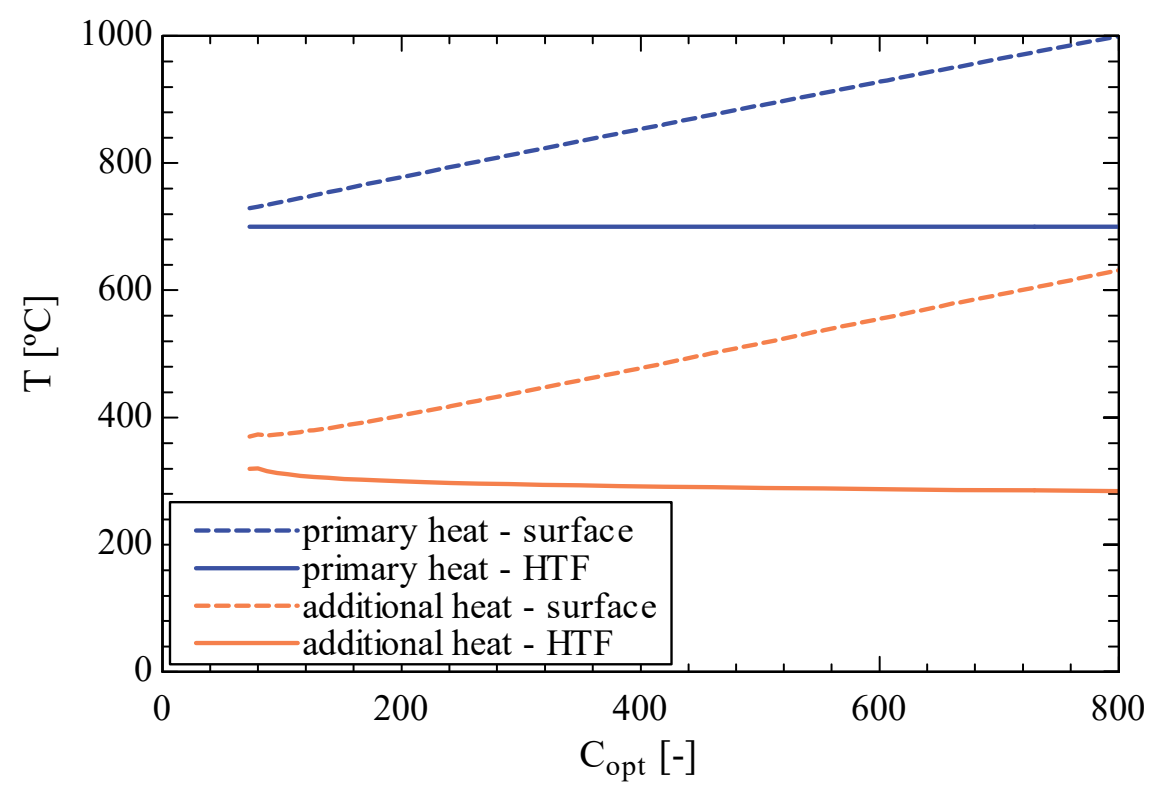

Figure 5.10. Maximum fluid and surface temperatures, $T$, in the sections of a Multi-Heating Solar Tower as a function of the optical concentration ratio, $C_{\text {opt }}$

The model of multi-heating solar tower will set the emissivity to $\varepsilon=0.85$ (Pyromark ${ }^{\circledR}$ ) if the surface temperature is above $500{ }^{\circ} \mathrm{C}$ and to $\varepsilon=0.15$ (cermet coatings) if it is below $500{ }^{\circ} \mathrm{C}$. This new version of the MHST will be called MHSTv2. Figure 5.11 shows the effect of varying the concentration ratio in the additional-heat section of a MHSTv2. The receiver efficiencies, $\eta_{\text {rec }}$, of the MHSTv2 and its two sections (primary heat and additional heat) are represented as a function of the concentration ratio in the additional-heat section, $C_{\text {opt,ah }}$.

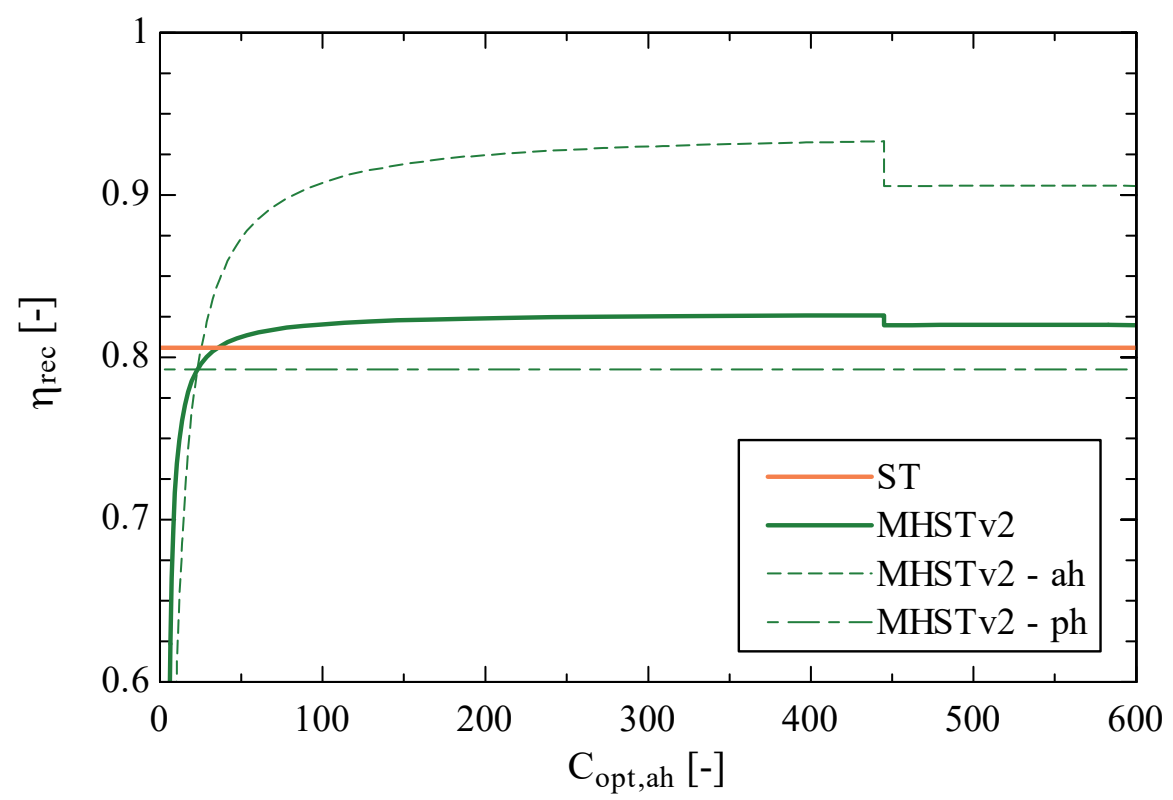

Figure 5.11. Receiver efficiency, $\eta_{r e c}$, of MHSTv2 and its two sections, primary heat (ph) and additional heat (ah), as a function of the concentration ratio for the additional-heat section, $C_{o p t, a h}$, keeping constant the concentration ratio in the primary-heat section at $C_{\text {opt }, p h}=600$ 
The optical concentration ratio is set to $C_{o p t, p h}=600$ in the primary-heat section. Thus, the receiver efficiency of this section remains constant. The MHSTv2 efficiency follows the behavior of the additional-heat efficiency: it rapidly increases at low concentrations and then asymptotes at concentration ratios around $C_{\text {opt,ah }}=100$. The receiver efficiency increases slowly from this value onwards till a concentration ratio $C_{o p t, a h}=445$. At this value, the emissivity raises from $\varepsilon=0.15$ to $\varepsilon=0.85$ as the surface temperature overpass $500^{\circ} \mathrm{C}$. The result is an abrupt decrease in efficiency in the addition-heat section and, therefore, in the MHSTv2. This abrupt decrease allows to see the improvement in efficiency thanks to the change of coating.

The efficiency of a solar tower is also represented in Figure 5.11. The efficiency of the multiheating solar tower is higher than the efficiency of the solar tower when the concentration ratio in the additional-heat is greater than $C_{o p t, a h}=35$. If the concentration ratio were $C_{o p t, a h}=445$, the relative increase in efficiency of the multi-heating solar tower with respect to the solar tower would be $\Delta \eta_{r e c}=2.5 \%$. Moreover, the thermal requirements of the multiheating solar tower are softer in the additional-heat section, which has the potential to reduce the complexity of the receiver and, therefore, the cost.

\subsection{Receiver and cycle system}

The Multi-Heating Solar Tower presents several advantages with respect to the standard Solar Tower. However, the receiver is only a part of the CSP plant. A Concentrating Solar Power plant is a system composed by two main subsystems, solar field and power block. This section analyses the efficiency of the Multi-Heating Solar Tower integrated with a multiheating cycle and compares it to the efficiency of a standard Solar Tower integrated with a simple regenerative cycle. Figure 5.12 and Figure 5.13 shows a scheme of these two types of CSP plants. Note that in the plant with multi-heating each heating has its own heat exchanger, receiver and set of heliostats, while in the plant with only one heating all these elements work exclusively for the primary heat.

This section focuses specifically on the integration of receiver and power block. The field of heliostats (the other part of the solar field that is not the receiver) is set aside. In this way, the concentrators can be designed afterwards according to the concentration needed by the receiver. The following section will focus on this matter. 


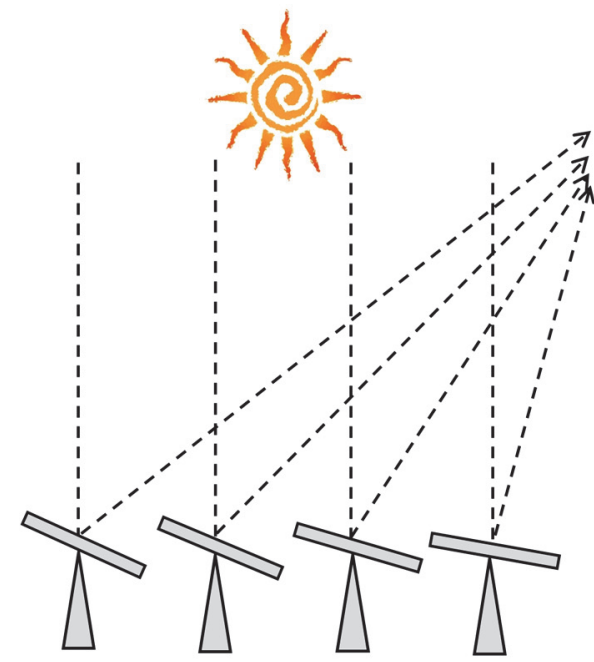

Heliostats

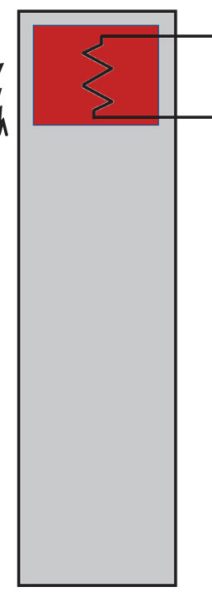

Solar Tower

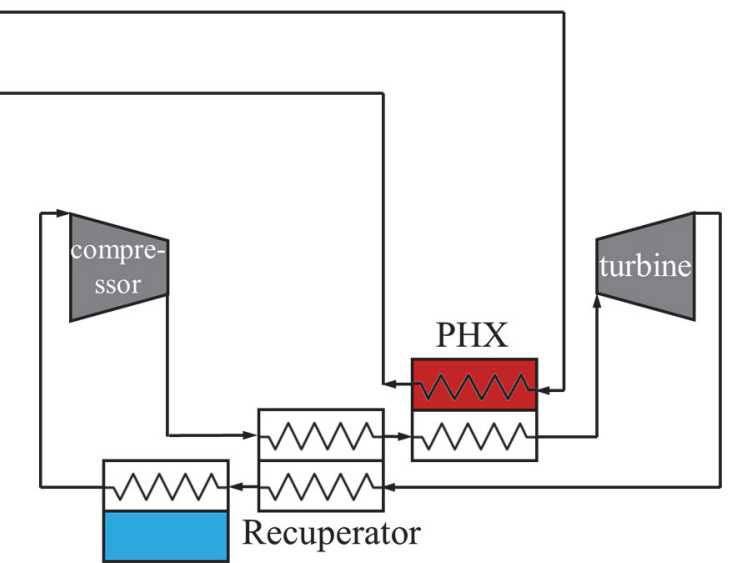

Precooler

Figure 5.12. CSP plant with standard Solar Tower and simple regenerative cycle

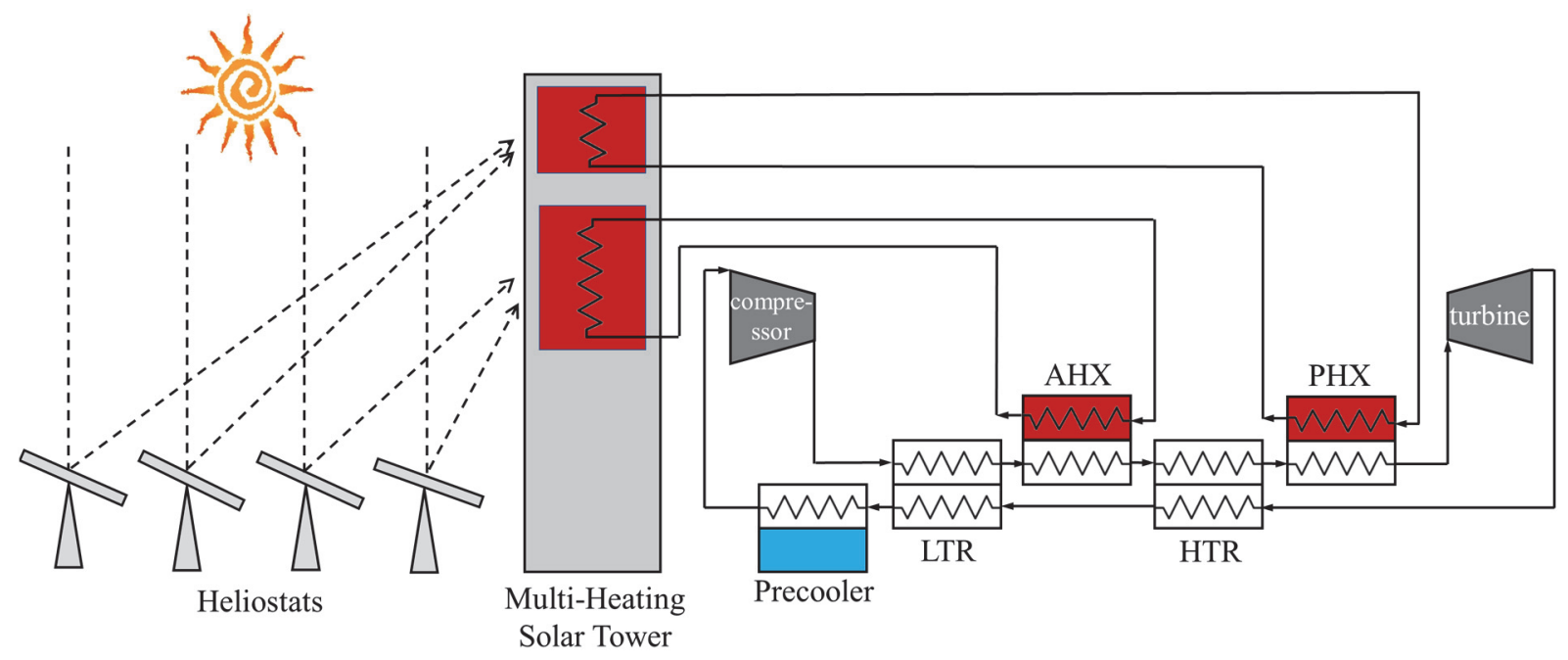

Figure 5.13. CSP plant with Multi-Heating Solar Tower and multi-heating cycle

The efficiency of the system resulting from the integration of receiver and power block is defined in Equation 5.15 as a function of the cycle thermal efficiency, $\eta_{t h}$, and the receiver efficiency, $\eta_{\text {rec. }}$

$$
\eta_{s y s t}=\eta_{t h} \eta_{r e c}
$$

Cycle thermal efficiency, $\eta_{t h}$, has been defined in Equation 2.73, and receiver efficiency, $\eta_{\text {rec }}$, in Equation 5.13. Anyway, they are expressed again in Equations 5.16 and 5.17 in order to unify the terminology.

$$
\eta_{t h}=\frac{\dot{W}_{t}-\dot{W}_{c}}{\sum \dot{Q}_{i}}
$$




$$
\eta_{\text {rec }}=\frac{\sum \dot{Q}_{i}}{\sum \frac{\dot{Q}_{i}}{\eta_{r e c, i}}}
$$

Note that although the thermal powers $\dot{Q}_{i}$ are the same in Equations 5.16 and 5.17., the fluid is different. The HTF supplies this power in the receiver to the $\mathrm{CO}_{2}$ in the cycle.

The parameters defining the cycle and the receiver have been explained in previous sections. The cycles will be defined by means of the effectiveness method with an effectiveness $\varepsilon=0.99$ and the conditions from Table 4.1. The parameters of the receiver are shown in Table 5.1. The parameters not shown in these tables will be varied in the course of the analysis.

\subsubsection{Temperature analysis}

Previous section studied the efficiency of solar tower receivers. Although the parameter analyzed was the receiver efficiency, the cycle was implicitly taken into account because it determined the thermal power needed and its range of temperatures. However, the cycle efficiency was not analyzed in order to focus the study on the receiver efficiency. This section studies the roll of the cycle together with the receiver. Three systems will be analyzed: simple cycles coupled to solar towers and multi-heating cycles coupled to multi-heating solar towers version 1 and version 2. Version 1 will have two sections at different temperatures with the same concentration ratio in both. Version 2 will have two sections at different temperatures but also with different concentration ratios. The same concentration ratio will be applied to the standard solar tower, the multi-heating solar tower version 1 and the primary-heat section of the multi-heating solar tower version 2. The concentration ratio of the additional-heat section of the version 2 will be optimized to maximize the efficiency.

Figure 5.14 shows the efficiencies of thermodynamic cycles and solar receivers that have been previously studied as a function of the turbine inlet temperature, $T_{t, i n}$. The cycles are wetcooled with heat exchanger effectiveness $\varepsilon=0.99$ and a low-side pressure $P_{L}=7.21 \mathrm{MPa}$. Under these conditions, the cycle efficiency is the same in simple cycles (SC) and multiheating cycles (MHC), as it was seen in Chapter 3, and it increases with the turbine inlet temperature. The efficiency is different for the three presented receivers. The efficiency of MHSTv2 will always be the highest and the ST efficiency the lowest. Unlike cycle efficiency, receiver efficiency decreases with the turbine inlet temperature. The opposing effects of cycle and receiver efficiencies will result in a system efficiency curve with a maximum. This maximum will be achieved at a different turbine inlet temperature depending on the system. 


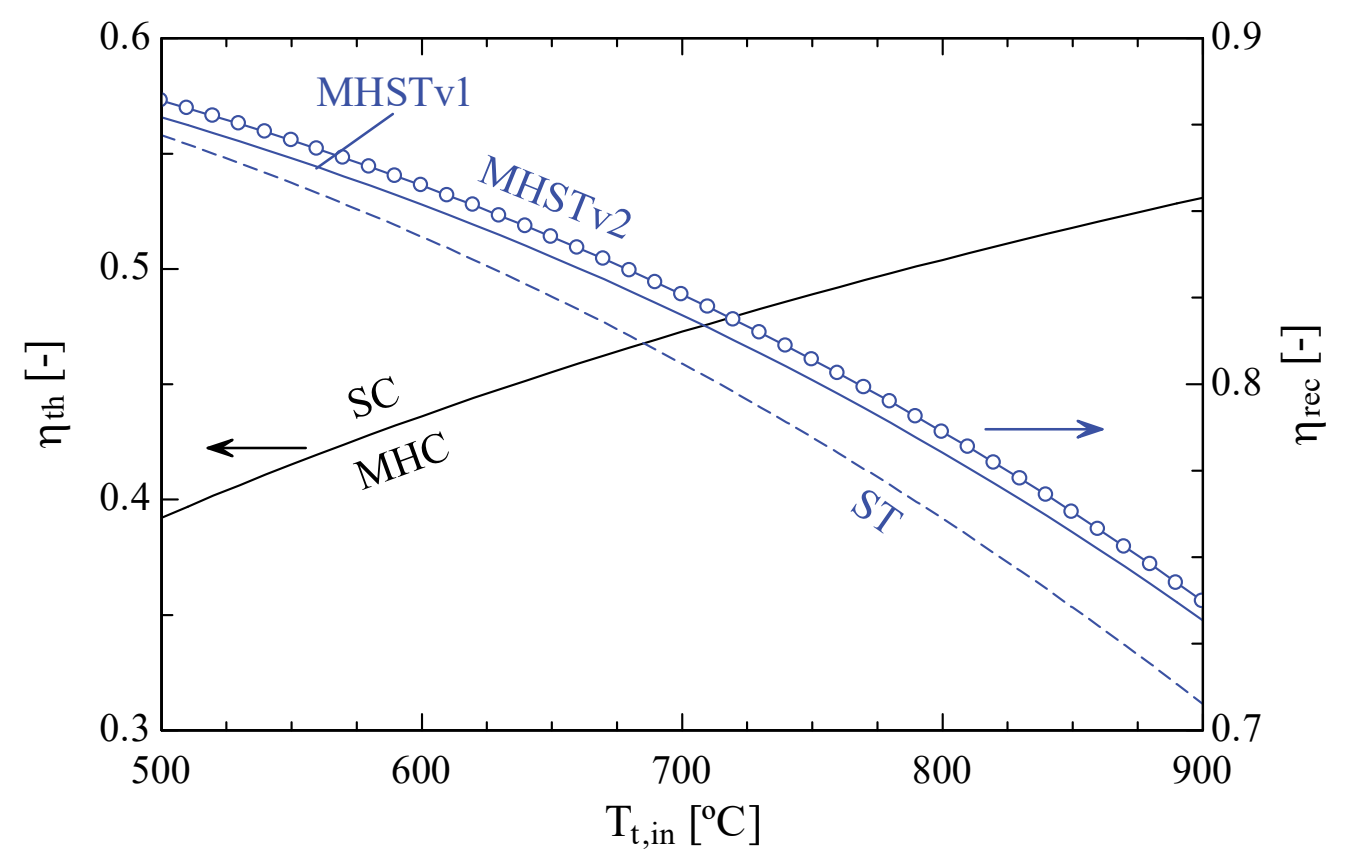

Figure 5.14. Efficiencies of thermodynamic cycles $\eta_{\text {th }}$ and receivers $\eta_{r e c}$ as a function of the turbine inlet temperature, $T_{t, i n}$. Cycles: simple (SC) and multi-heating (MHC). Receivers: Solar Tower (ST), Multi-Heating Solar Towers version 1 and version 2 (MHSTv1 and MHSTv2)

Figure 5.15 shows the efficiency of the three systems resulting from the integration of cycles and receivers from Figure 5.14: SC+ST (simple cycle coupled to Solar Tower), MHC+MHSTv1 (multi-heating cycle coupled to multi-heating solar tower v1) and MHC+MHSTv2 (multi-heating cycle coupled to multi-heating solar tower v2). In the case of the $\mathrm{SC}+\mathrm{ST}$ system, the optimum turbine inlet temperature is $T_{t, i n}=775{ }^{\circ} \mathrm{C}$, while in the case of MHC+MHSTv2 the optimum turbine inlet temperature raises up to $T_{t, i n}=800{ }^{\circ} \mathrm{C}$. The systems with multi-heating cycle, besides having a greater optimum turbine inlet temperature, have greater efficiency. The difference in efficiency between systems with multi-heating and simple cycles increases at higher turbine inlet temperatures.

The results shown in Figure 5.14 and Figure 5.15 have been obtained for a constant low-side pressure $P_{L}=7.21 \mathrm{MPa}$. However, the results will vary if the low-side pressure is optimized to maximize the system efficiency, especially in simple cycles. The system efficiency will be calculated by optimizing the low-side pressure for the cases of wet- and dry-cooled cycles. In the case of dry-cooled cycles, previous chapter showed that high effectiveness could lead to very big heat exchangers. Thus, the low-side pressure will be kept below the discontinuity line to avoid this. 


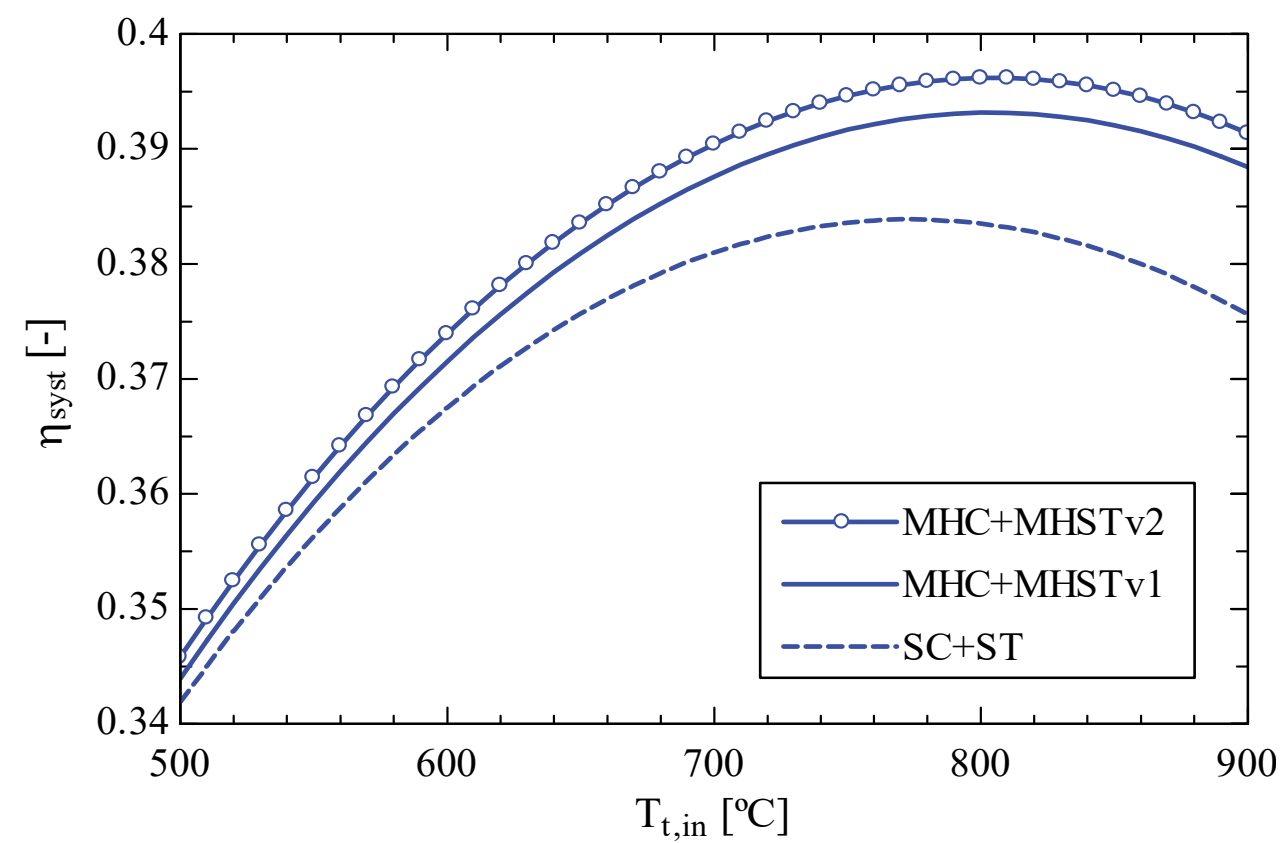

Figure 5.15. Efficiencies of systems SC+ST (simple cycle coupled to Solar Tower) and MHC+MHSTv1 and MHC+MHSTv2 (Multi-Heating cycle coupled to Multi-Heating Solar

Tower versions 1 and 2)

Figure 5.16 shows the system efficiency and the optimum pressure ratio of the systems $\mathrm{SC}+\mathrm{ST}, \mathrm{MHC}+\mathrm{MHSTv1}$ and MHC+MHSTv2 at wet- and dry-cooled conditions. The optimum pressure ratio in MHC+MHSTv1 and MHC+MHSTv2 systems is the same. The pressure ratio is calculated for the MHC+MHSTv1 system and then used in MHC+MHSTv2. The pressure ratio tends to increase with the turbine inlet temperature in dry cooled cycles. This tendency is interrupted in wet-cooled cycles by the discontinuity line. In the case of simple wet-cooled cycles, the pressure ratio increases again at turbine inlet temperatures above $T_{t, i n}=740{ }^{\circ} \mathrm{C}$, and in the case of multi-heating wet-cooled cycles, the pressure ratio remains constant for the whole range of temperatures represented in Figure 5.16. In all the cases, the optimum pressure ratio of multi-heating cycles is equal or less than the optimum pressure ratio of simple cycles. For example, in the reference case with turbine inlet temperature $T_{t, i n}=700{ }^{\circ} \mathrm{C}$, the pressure ratio of multi-heating cycles is $7 \%$ smaller than in simple cycles when there is dry-cooling, and it is the same when there is wet-cooling.

The system efficiency $\eta_{\text {syst }}$ presents the same behavior in all the cases: there is an optimum turbine inlet temperature for which the maximum system efficiency is achieved. This temperature is different depending on the case, but all of them are in the range between $800{ }^{\circ} \mathrm{C}$ and $830^{\circ} \mathrm{C}$. Raising the turbine inlet temperature above these values will imply a decrease in efficiency with a more expensive system (higher temperatures involve higher 
costs). Thus, the turbine inlet temperature in an optimum system configuration should be below $800^{\circ} \mathrm{C}$. A balance between system efficiency and cost would evaluate the best configuration for turbine inlet temperatures in the raising part of the curve, such as the reference case with turbine inlet temperature $T_{t, i n}=700{ }^{\circ} \mathrm{C}$.

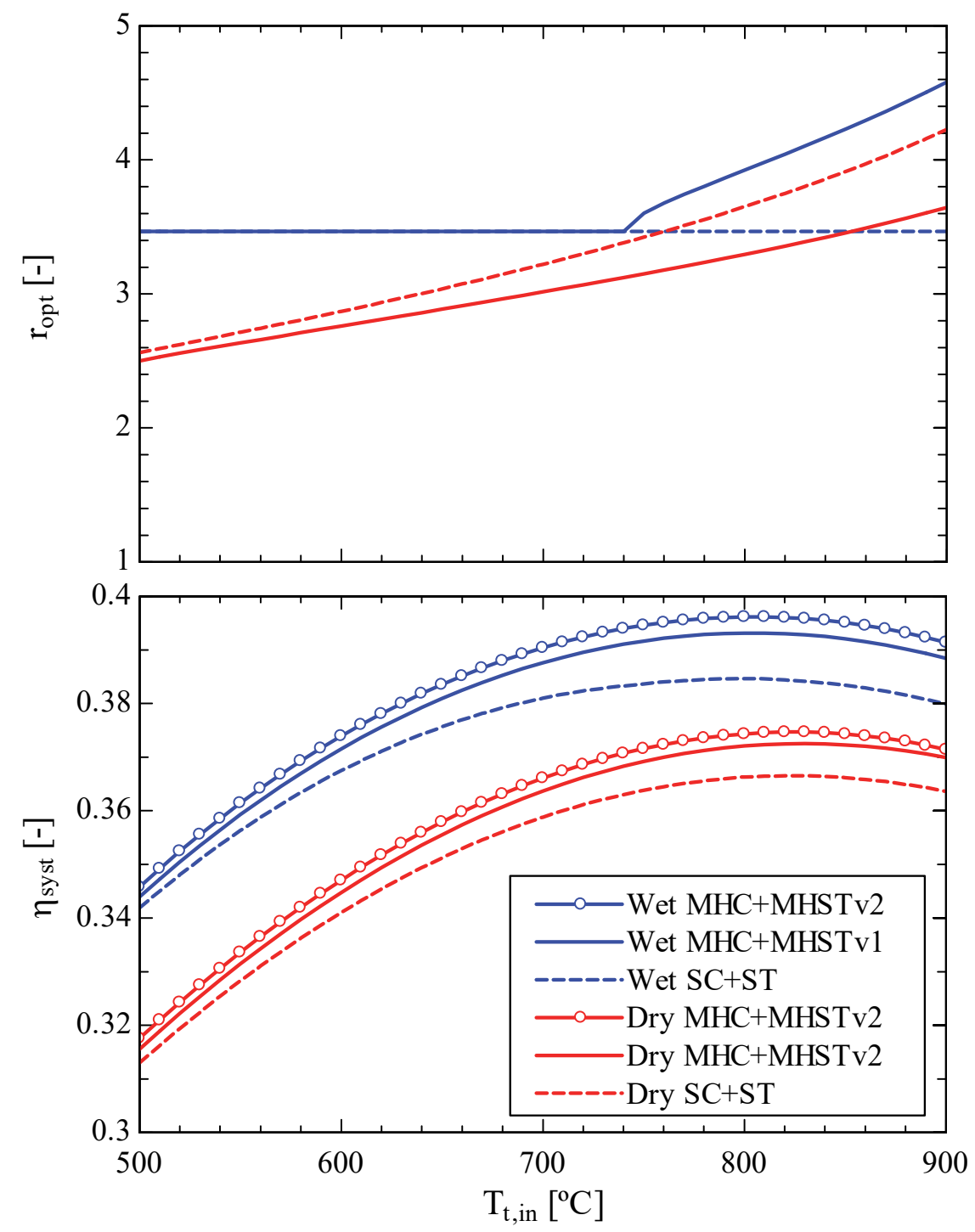

Figure 5.16. Optimum pressure ratio, $r_{\text {opt }}$, and system efficiency, $\eta_{\text {syst }}$, as a function of the turbine inlet temperature, $T_{t, i n}$, for systems SC+ST (Simple cycle coupled to Solar Tower) and MHC+MHST (Multi-Heating cycle coupled to Multi-Heating Solar Tower) under wet- and dry-cooled conditions

The efficiencies in systems with dry-cooled cycles are lower than in systems with wet-cooled cycles, and the benefit of multi-heating is also smaller. Figure 5.17 shows the relative increase in system efficiency, $\Delta \eta_{\text {syst }}$, of MHC+MHSTv2 with respect to $\mathrm{SC}+\mathrm{ST}$ as a function of the turbine inlet temperature, $T_{t, i n}$. The benefit of multi-heating increases with the turbine inlet temperature till temperatures around $800{ }^{\circ} \mathrm{C}$. From these temperatures onwards the increase in 
system efficiency is more or less constant. The relative increase in wet-cooled cycles is more sensitive to the turbine inlet temperature, it varies from $1 \%$ to $3 \%$, while in dry-cooled cycles is in between $1.5 \%$ and $2 \%$. In the reference case with turbine inlet temperature $T_{t, i n}=700{ }^{\circ} \mathrm{C}$ the relative increase of efficiency is $\Delta \eta_{\text {syst }}=2.5 \%$ in wet-cooled conditions and $\Delta \eta_{\text {syst }}=2 \%$ in dry-cooled conditions.

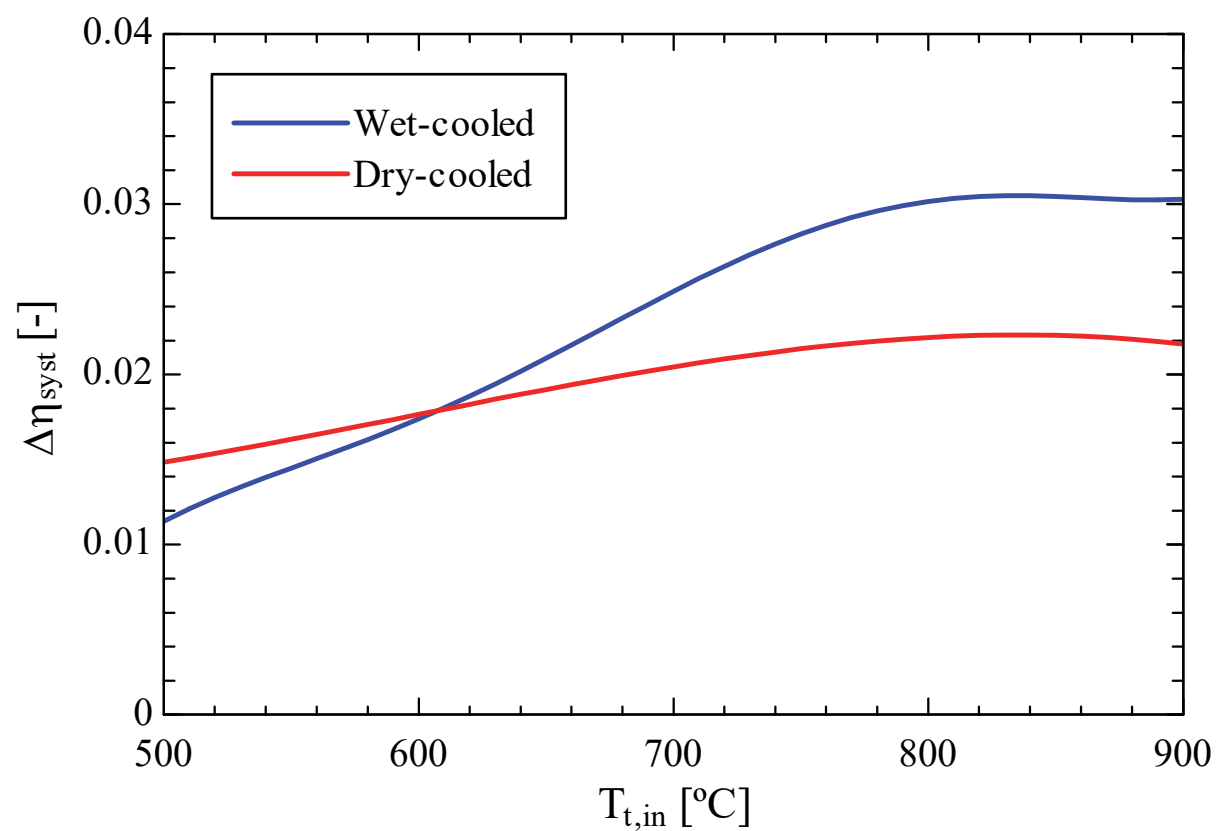

Figure 5.17. Relative increase in system efficiency, $\Delta \eta_{\text {syst }}$, of multi-heating with respect to simple cycles as a function of the turbine inlet temperature, $T_{t, i n}$

The results shown in this section have been obtained for a concentration ratio $C_{\text {opt }}=600$ in all the receivers except in the additional-heat section of multi-heating solar towers v2. Figure 5.16 shows that the optimum turbine inlet temperatures for this concentration ratio are in between $800{ }^{\circ} \mathrm{C}$ and $830^{\circ} \mathrm{C}$, depending on the system. The turbine inlet temperature of an optimal configuration should not overpass these values. If other concentrations were used, other ranges of temperature would be obtained. Adapting fluid temperature and concentration is necessary to obtain the optimum system configuration.

\subsubsection{Concentration analysis}

A coherent design of a CSP system must match concentration and turbine inlet temperature. In actual CSP plants, the operational limit is generally imposed by the maximum temperature of the HTF. In the reference system proposed in this chapter, the maximum heat transfer temperature will be $T_{H T F}=725{ }^{\circ} \mathrm{C}, 25^{\circ} \mathrm{C}$ above the turbine inlet temperature $T_{t, \text { in }}=700{ }^{\circ} \mathrm{C}$. Thus, it is important to analyze the system for this temperature. 


\subsubsection{Analysis of the main concentration ratio}

Figure 5.18 analyses the influence of the concentration ratio, $C_{o p t}$, in the system performance, $\eta_{\text {syst }}$, depending on the type of system. The concentration ratio $C_{\text {opt }}$ in MHC+MHSTv2 systems establishes the concentration ratio of the primary-heat section and the maximum concentration ratio allowed in the additional-heat section. The concentration in the additionalheat section is reduced in order to decrease the additional-heat surface temperature to $500{ }^{\circ} \mathrm{C}$ and maximize the receiver efficiency.

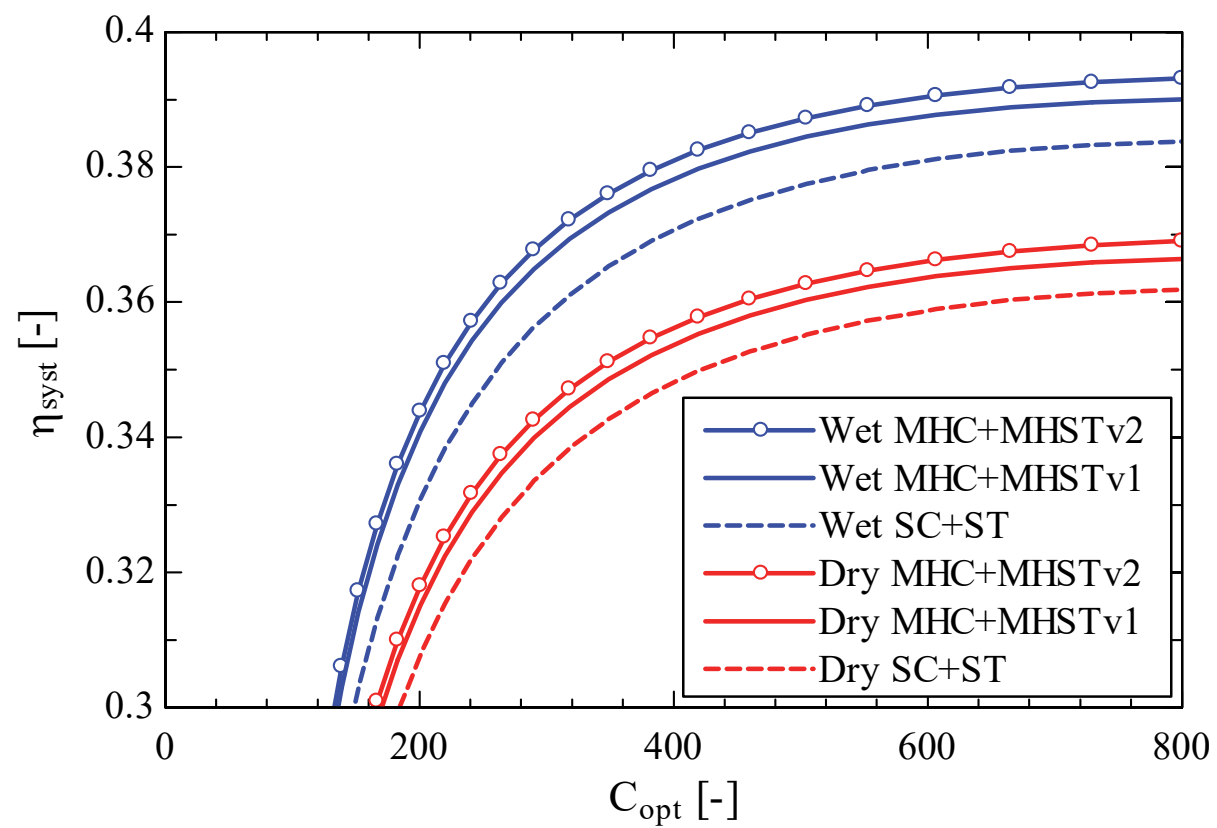

Figure 5.18. System efficiency, $\eta_{\text {syst }}$, as a function of the optical concentration ratio, $C_{o p t}$, for systems SC+ST (Simple cycle coupled to Solar Tower) and MHC+MHST (Multi-Heating cycle coupled to Multi-Heating Solar Tower) under wet- and dry-cooled conditions

The pressure ratio in each cycle is the one shown in Figure 5.16 for the turbine inlet temperature $T_{t, \text { in }}=700^{\circ} \mathrm{C}$. This means that the pressure ratio in each system is constant regardless of the concentration ratio. The pressure ratio is not optimized for each concentration in order to focus the analysis on the rest of parameters.

The shape of the system efficiency $\eta_{s y s t}$ in Figure 5.18 is ruled by the receiver efficiency. It increases rapidly at low concentration ratios and asymptotes towards its highest efficiency at high concentration ratios. The asymptotes are reached at similar concentration ratios in all the systems. According to Equation 5.14, all the asymptotes in Figure 5.18 start at concentration ratios between $C_{o p t}=550$ and $C_{o p t}=600$. These values would be different if the turbine inlet temperature varied. 
The difference in efficiency between systems is more or less constant regardless of the concentration ratio. Figure 5.19 shows the relative increase in system efficiency, $\Delta \eta_{\text {syst }}$, of $\mathrm{MHC}+\mathrm{MHST} 2$ systems with respect to $\mathrm{SC}+\mathrm{ST}$ systems as a function of the optical concentration ratio, $C_{\text {opt }}$. The relative increase is slightly higher under wet-cooled conditions. For concentration ratios above $C_{\text {opt }}=400$, the increase is around $2.6 \%$ in wet-cooled cycles and $2.1 \%$ in dry-cooled cycles. For lower concentration ratios, the same increase in efficiency involves a larger relative increase, but because the system efficiency is lower.

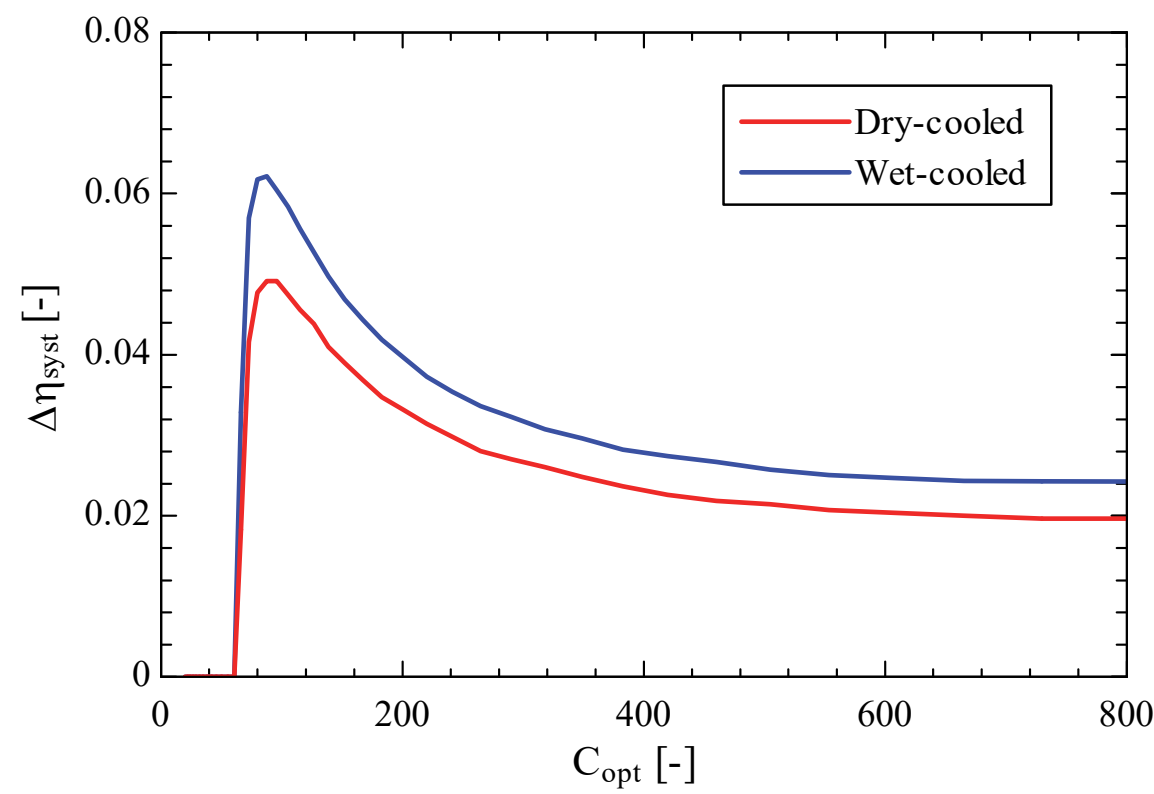

Figure 5.19. Relative increase in system efficiency, $\Delta \eta_{\text {syst }}$, of multi-heating with respect to simple cycles as a function of the optical concentration ratio, $C_{\text {opt }}$

\subsubsection{Analysis of the additional-heat concentration ratio}

In the reference system with turbine inlet temperature $T_{t, i n}=700{ }^{\circ} \mathrm{C}$ and concentration ratio $C_{\text {opt }}=600$, the relative increase in efficiency of the MHC+MHSTv2 system with respect to $\mathrm{SC}+\mathrm{ST}$ is $\Delta \eta_{\text {syst }}=2.5 \%$ in wet-cooled conditions and $\Delta \eta_{\text {syst }}=2 \%$ in dry-cooled conditions. The maximum temperature in the additional-heat of the multi-heating cycle is $287^{\circ} \mathrm{C}$ under wet-cooled conditions and $322{ }^{\circ} \mathrm{C}$ under dry-cooled conditions, and the maximum concentration ratios in this section have been calculated to not overpass $500{ }^{\circ} \mathrm{C}$ in the receiver surface.

The influence of the concentration ratio in the additional-heat section, $C_{\text {opt,ah }}$, is shown in Figure 5.20. The figure compares the system efficiencies for the cases of solar tower and simple cycle (dashed line) and multi-heating solar tower v2 with multi-heating cycle (continuous line). Solar tower values are independent of $C_{o p t, a h}$, but they are shown as 
reference for the comparison. Concentration ratios over $C_{o p t, a h}=445$ in wet-cooled cycles and over $C_{o p t, a h}=365$ in dry-cooled cycles are not represented because the surface temperature is above $500{ }^{\circ} \mathrm{C}$. These maximum concentration ratios maximize the system efficiency. However, lower values will also be a potential solution as the required concentrators will probably be simpler.

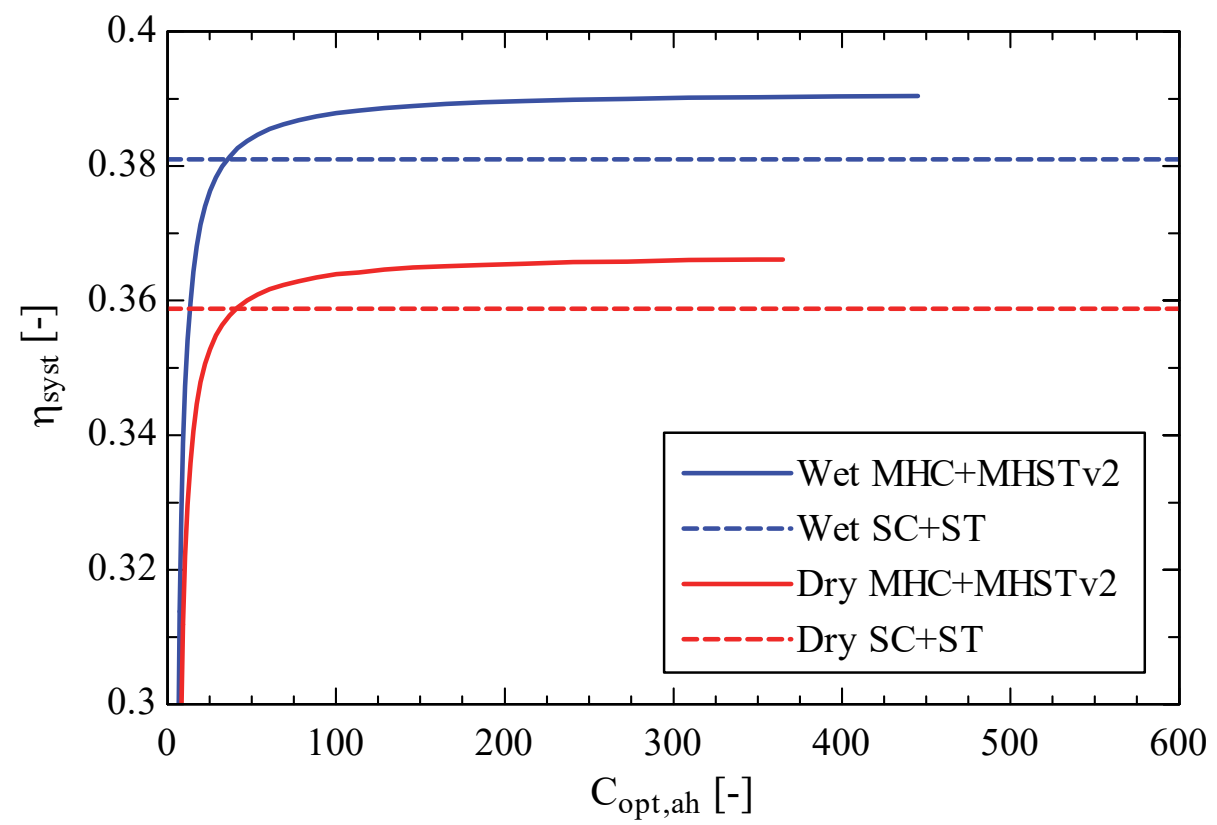

Figure 5.20. System efficiency, $\eta_{\text {syst }}$, as a function of the additional-heat concentration ratio, $C_{\text {opt }, a h}$, for systems SC+ST (Simple cycle coupled to Solar Tower) and MHC+MHSTv2 (Multi-Heating cycle coupled to Multi-Heating Solar Tower v2) under wet- and dry-cooled conditions

The efficiency of the MHC+MHSTv2 system is higher than the efficiency of the SC+ST system when the additional-heat concentration ratio is greater than $C_{o p t, a h}=35$ under wetcooled conditions and $C_{\text {opt,ah }}=41$ under dry-cooled conditions. Increasing the concentration ratio above these values involve greater benefits of the multi-heating. The surface of receiver and heliostats will decrease at the expense of stronger thermal requirements and more complex concentrators.

Figure 5.21 shows the receiver surface as a function of the additional-heat concentration ratio for systems $\mathrm{SC}+\mathrm{ST}$ and MHC+MHSTv2 under wet- and dry-cooled conditions. The areas of the $\mathrm{SC}+\mathrm{ST}$ system do not depend on $C_{o p t, a h}$, but they are shown as reference for the comparison. In the system MHC+MHSTv2, the higher the concentration ratio $C_{o p t, a h}$ the smaller the areas are. 


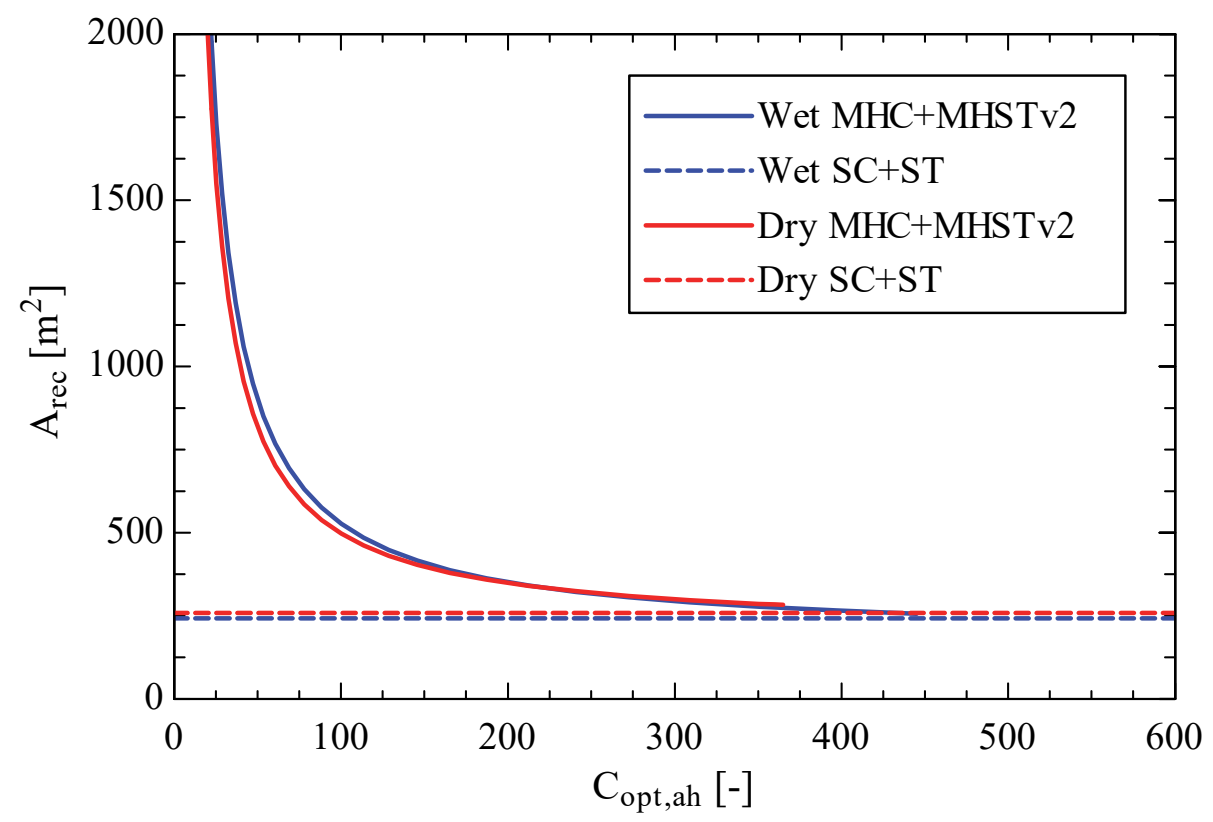

Figure 5.21. Receiver surface, $A_{r e c}$, as a function of the additional-heat concentration ratio,

$$
C_{\text {opt,ah }}
$$

Receiver areas at high concentration ratios are almost the same in systems with and without multi-heating. When the concentration ratio is reduced, the receiver area increases. It increases slowly at high concentration ratios and rapidly when the concentration ratios are small. For example, the receiver areas of simple and multi-heating systems are almost the same at concentration ratios close to $C_{\text {opt,ah }}=400$, the receiver area of the multi-heating solar tower v2 is twice the area of the Solar Tower when the concentration ratio is $C_{\text {opt,ah }}=100$, and 8 times larger when the concentration ratio is $C_{o p t, a h}=25$.

The option to have a tower-like receiver with an area twice the area of a standard solar tower should not involve any problems. However, too large receiver areas resulting from low concentration ratios could imply that the surface does not fit into a solar tower-like receiver. In this case, an option could be to divide the heats into two types of receivers: the primary heat in a Solar Tower and the additional heat in a linear receiver, which has the advantage of being cheaper in spite of the larger required areas.

The system with multi-heating cycle and multi-heating solar tower v2 does not only achieve higher efficiencies, but also have lower thermal requirements and needs smaller optical concentration ratios. The higher efficiencies imply smaller areas of concentrators, which is the main cost of a Concentrating Solar Power plant. The next section studies the field of concentrators, which has not been analyzed yet. 


\subsection{The solar field}

A Concentrating Solar Power Plant is a system composed by two main subsystems, solar field and power block. Previous section has analysed the integration between part of the solar field (the receiver) and power block. Once the conditions of heat flux in the receiver are known, the concentrators field (the other part of the solar field that is not the receiver) can be designed accordingly. The purpose of this section is not to design a specific concentrator, but to show the effect of the concentrator in CSP systems, and present different options for multi-heating receivers.

The efficiency of a CSP plant resulting from the integration of solar field and power block is defined in Equation 5.18 as a function of the solar field efficiency, $\eta_{S F}$ and the cycle thermal efficiency, $\eta_{t h}$.

$$
\eta_{C S P}=\eta_{S F} \eta_{t h}
$$

Cycle thermal efficiency, $\eta_{t h}$, has been defined in Equation 5.16. The solar field efficiency $\eta_{S F}$ is defined in Equation 5.19 as the ratio between the thermal power absorbed by the HTF, $\dot{Q}$, and the solar power reaching the concentrators, $\dot{Q}_{S F}$.

$$
\eta_{S F}=\frac{\sum \dot{Q}_{i}}{\sum \dot{Q}_{S F, i}}=\frac{\sum \dot{Q}_{i}}{\sum \frac{\dot{Q}_{r e c, i}}{\eta_{o p t, i}}}=\frac{\sum \dot{Q}_{i}}{\sum \frac{\dot{Q}_{i}}{\eta_{o p t, i} \eta_{r e c, i}}}
$$

Note that the definition of solar field efficiency is similar to the definition of receiver efficiency (Equation 5.17), but adding the concentration efficiency $\eta_{\text {opt }}$, defined in Equation 5.1 as the ratio between the solar power impinging on the receiver and the solar power reaching the concentrators. The definition of solar field efficiency considers that each section of the multi-heating receiver have different concentration ratio and temperature and, therefore, different receiver efficiency $\eta_{r e c, i}$. It also considers that the mirrors used to concentrate the solar power in each section may have a different optical efficiency $\eta_{o p t, i}$.

\subsubsection{Optical efficiency}

The optical concentration ratio $C_{o p t}$ has been used in the last sections in order to set apart the influence of optical efficiency on system efficiency. However, the optical concentration is the result of a geometrical concentration ratio $C_{g e o}$ and an optical efficiency $\eta_{\text {opt }}$. For example, the optical concentration ratio used in solar towers has been $C_{o p t}=600$, which is the result of a 
geometrical concentration ratio $C_{g e o}=1000$ and an optical efficiency $\eta_{o p t}=0.6$ (Padilla et al., 2015a). This optical efficiency is the average of a field of heliostats in a common CSP plant with solar tower. Figure 5.22 shows an example of the distribution of these optical efficiencies along a field of heliostats.

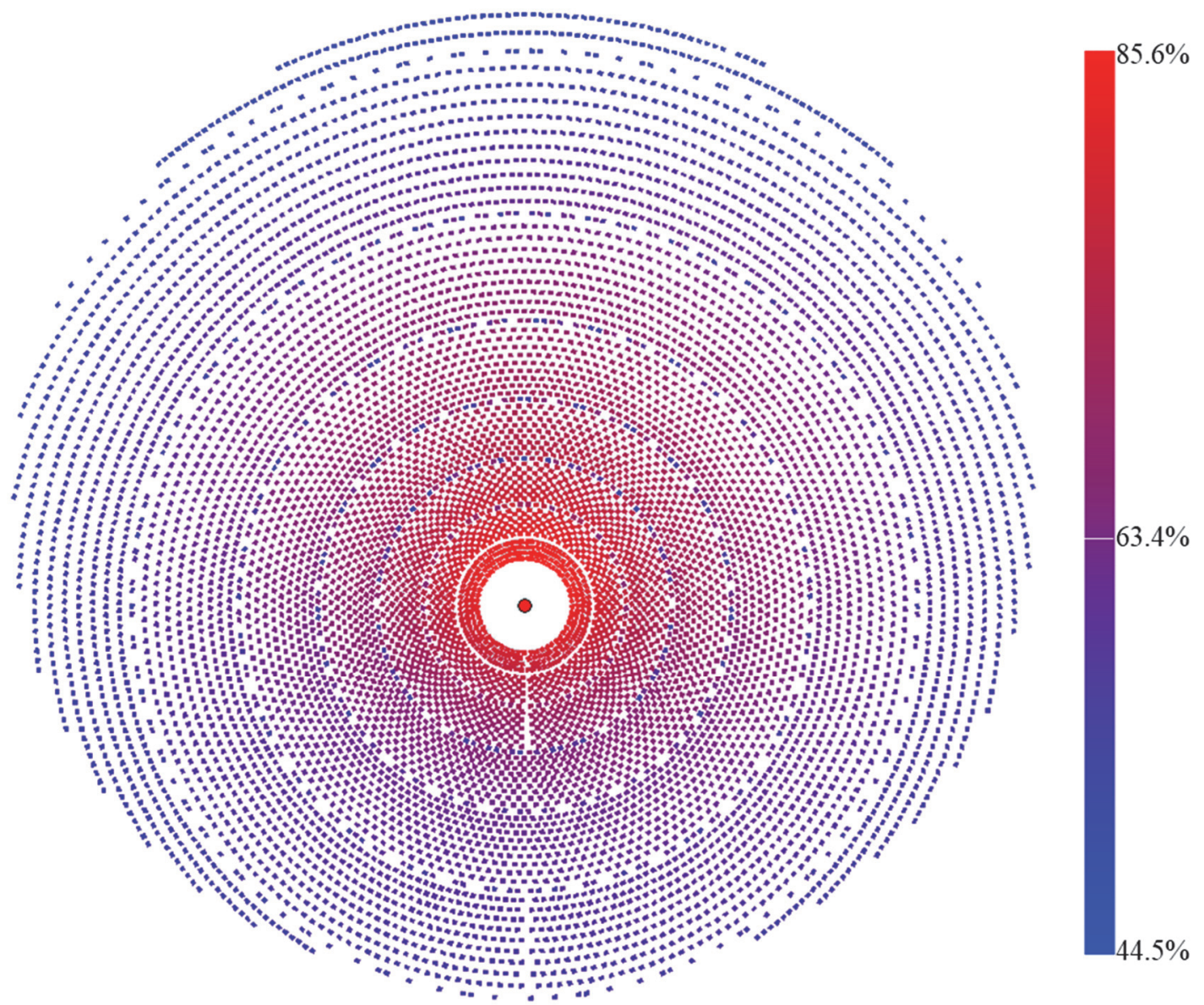

Figure 5.22. Optical efficiency of heliostats in a system with Solar Tower obtained with the software solarPILOT (Wagner and Wendelin, 2018)

An optical efficiency $\eta_{\text {opt }}=0.6$ will be used for the standard solar tower and for the primaryheat section of the multi-heating solar tower due to the similarities with the standard solar tower. However, the optical efficiency of the additional-heat section could be different depending on the heliostats selected to impinge the solar heat flux into the receiver area. Three options are proposed for the composition of the pair concentrator-receiver in the additional-heat section:

- Linear receiver as additional heat. This option would use linear concentrators such as PTC, with higher optical efficiency, for the additional heat. The space surrounding the tower could be used for this purpose. This option would mix different types of 
concentrators. In fact, the option of PTC could be substituted by other type of concentrator designed ad hoc for this specific case of multi-heating receiver.

- Internal heliostats for the additional-heat section. This option would use heliostats close to the tower, with higher optical efficiency, for the additional heat. The space surrounding the tower in Figure 5.22 does not contain heliostats because they cannot reflect the solar radiation into the top part of the tower, where the receiver surface is. However, a larger area extended along the tower (area needed by the additional-heat section) could be heated by heliostats located in the empty space close to the tower.

- External heliostats for the additional-heat section. This option would use the furthest heliostats from the tower, with low optical efficiency, for the additional heat. The idea would be to increase the optical efficiency of these heliostats as the receiver area of the additional-heat section will be larger. Moreover, the heliostats used for the primary-heat section would get rid of the heliostats with the lowest optical efficiencies, and therefore its average efficiency would increase.

An analysis of the first two options will be done considering an optical efficiency $\eta_{\text {opt }}=0.7$ for the additional-heat section. The results will be compared with the case of optical efficiency $\eta_{\text {opt }}=0.6$.

\subsubsection{Analysis of the optical efficiency}

Figure 5.23 represents the efficiency of a CSP plant, $\eta_{C S P}$, as a function of the additional-heat concentration ratio, $C_{o p t, a h}$, for different CSP systems with solar tower (ST) and multi-heating solar tower v2 (MHSTv2). The subscript of the MHSTv2 system stands for the optical efficiency of the mirrors in charge of concentrating the solar power into the additional-heat section. The efficiency of the CSP plants SC+ST and MHC+MHSTv20.6 is the system efficiency shown in Figure 5.20 times the optical efficiency $\eta_{o p t}=0.6$. The relative increase in efficiency between MHC+MHSTv2 0.6 and $\mathrm{SC}+\mathrm{ST}$ are up to $\Delta \eta_{C S P}=2.5 \%$ under wet-cooled conditions and $\Delta \eta_{C S P}=2 \%$ under dry-cooled conditions.

The efficiency of the CSP plant with multi-heating solar tower assuming a higher optical concentration in the additional-heat section, i.e., the MHC+MHSTv20.7 system, increases considerably with respect to the MHC+MHSTv2 0.6 system. Thus, the relative increase with respect to the $\mathrm{SC}+\mathrm{ST}$ system also increases. In this case, it is up to $\Delta \eta_{C S P}=6.1 \%$ under wetcooled conditions and $\Delta \eta_{C S P}=4.7 \%$ under dry-cooled conditions. 


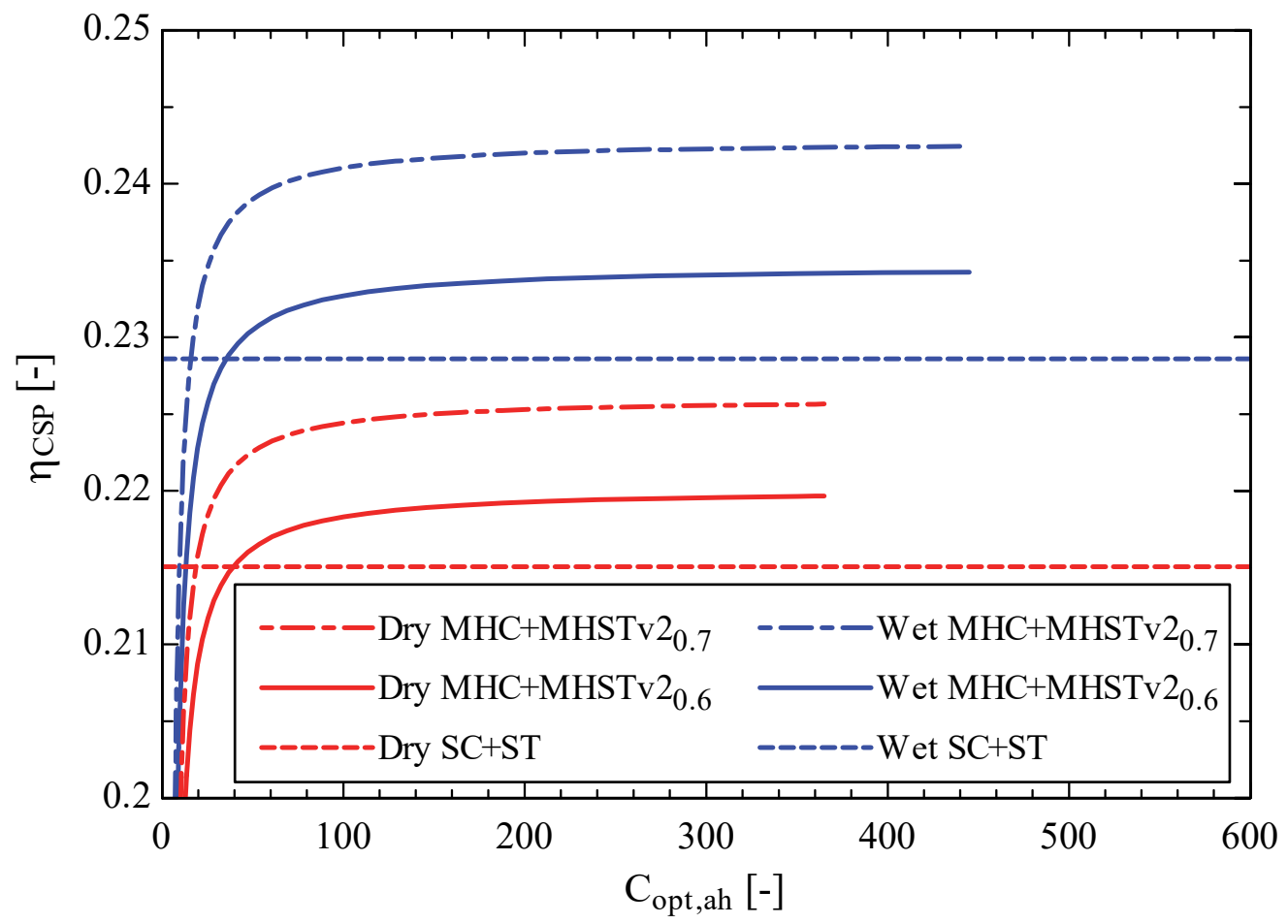

Figure 5.23. Efficiency of a CSP plant, $\eta_{C S P}$, as a function of the additional-heat concentration ratio, $C_{\text {opt }, a h}$. The subscript of the systems MHC+MHSTv2 stands for the optical efficiency of the concentration ratio in the additional-heat section.

The receiver areas of the systems MHC+MHSTv2 0.6 and MHC+MHSTv20.7 are the same if the same optical concentration ratios are employed. However, the area of mirrors will change. The analysis of efficiency of a CSP plant allows to calculate the area of mirrors required in the Solar Field. Figure 5.24 shows the area of heliostats, Ahel, required by the different systems as a function of the additional-heat concentration ratio, $C_{o p t, a h}$. This area is directly linked to the efficiency of the CSP plant. The area of heliostats is smaller in systems with multi-heating, especially if the optical efficiency of the additional-heat section is higher than in the primary-heat section.

If the optical efficiency of the additional-heat section is $\eta_{o p t}=0.7$, then even very low concentration ratios get to reduce the heliostats area in multi-heating systems. For example, an optical concentration ratio $C_{\text {opt }, a h}=42$, which would correspond to a modest geometrical concentration ratio $C_{g e o, a h}=60$, still get to reduce the heliostats surface a $4 \%$ under wetcooled conditions and $2.9 \%$ under dry-cooled conditions. This reduction is greater at higher concentration ratios although at the expense of a more complex concentrator. For example, the heliostats surface in a multi-heating system with concentration ratio in the additional-heat section $C_{o p t, a h}=300$ gets to be reduced $5.6 \%$ under wet-cooled conditions and $4.6 \%$ under dry-cooled conditions. 


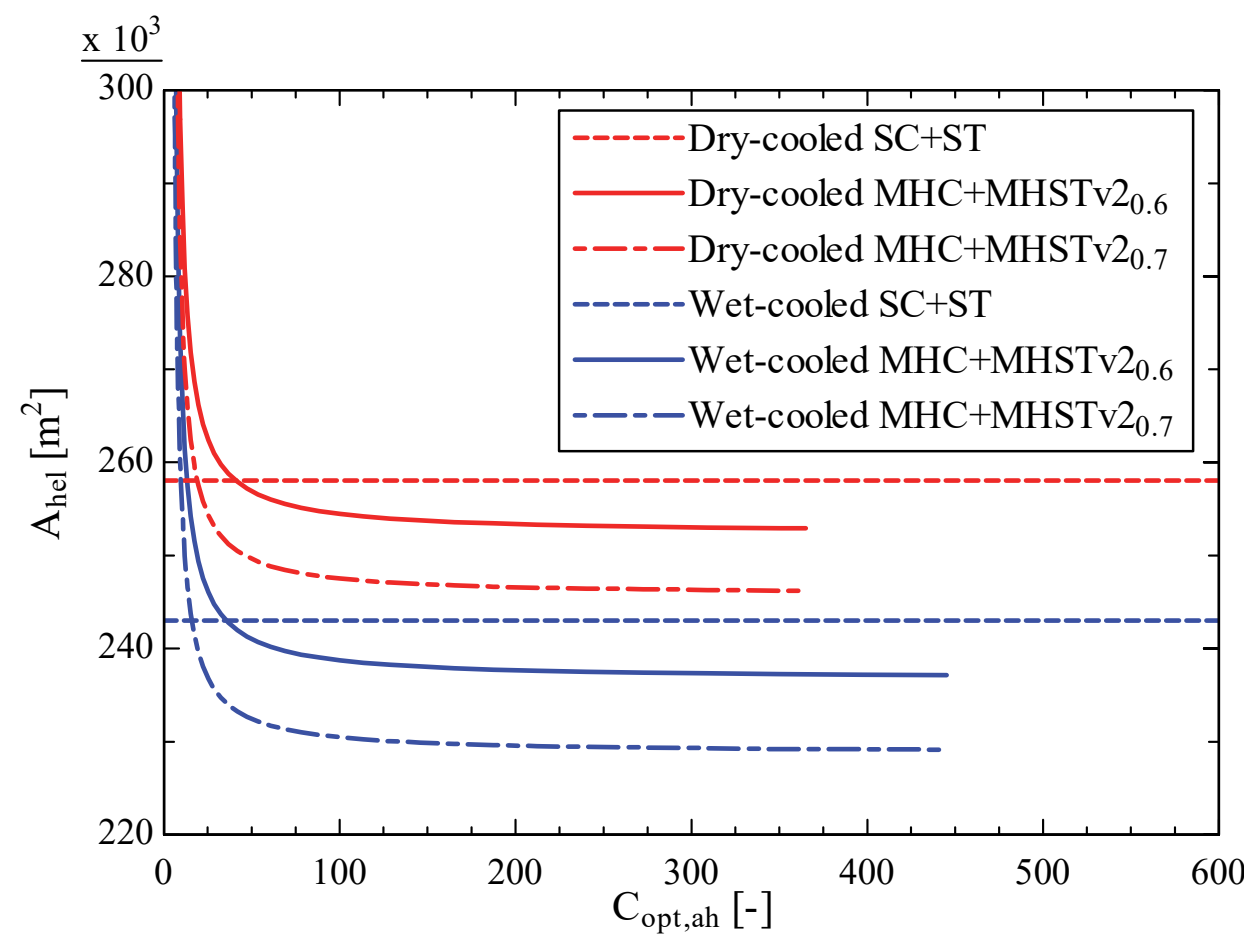

Figure 5.24. Heliostats surface, $A_{h e l}$, as a function of the additional-heat concentration ratio, $C_{\text {opt,ah. }}$ The subscript of the systems MHC+MHSTv2 stands for the optical efficiency of the concentration ratio in the additional-heat section.

The surface of heliostats is the most expensive part of a CSP plant. Thus, systems with multiheating solar tower, which achieve to reduce this surface, seem to be a good option to reduce the total cost of a CSP plant. However, these systems come together with multi-heating cycles with big intermediate heat exchangers. The balance between the savings of reducing the area of heliostats and the higher costs resulting from the larger heat exchangers will determine whether multi-heating systems are an improvement with respect to systems with simple cycles or not.

\subsection{Tecno-economic analysis}

The roadmap marked by the National Renewable Energy Laboratory (NREL) suggests drycooled $\mathrm{CO}_{2}$ power cycles coupled to solar towers as the future of CSP plants (Mehos et al., 2017). Nevertheless, there are still several options for the type of cycle and receiver. The options for the receiver depend mainly on the heat transfer fluid: molten salts, supercritical fluids and falling particles. The options for the cycles are mainly partial-cooling cycle and recompression cycles, although simple cycles have shown a high potential due to its simplicity and lower cost (Ho et al., 2015). 
The CSP plants proposed in this section integrate a multi-heating solar tower receiver with molten salts as HTF and a multi-heating cycle with $\mathrm{CO}_{2}$. The cost of this system will be compared with the cost of a solar tower with molten salts coupled to a simple regenerative cycle with $\mathrm{CO}_{2}$.

\subsubsection{Costs}

\subsubsection{Power block}

There are several studies about the efficiency of different pericritical cycle configurations with $\mathrm{CO}_{2}$ (commonly called supercritical $\mathrm{CO}_{2}$ Brayton cycles) and a few about drivers of cost such as equipment size. However, despite its importance, there is almost no complete technoeconomic analysis due to the lack of validated models for component costs. NREL and SNL are the main contributors to the cost estimation of these systems. The models proposed by these institutions (Carlson, Middleton and Ho, 2017; Ho et al., 2015; Neises and Turchi, 2019) will be used to estimate the cost of the power block.

The models are a mixture between existing cost models from literature for each major component and vendor cost data. The cost of the turbomachinery is expressed as a function of the power, $\dot{W}$, and the cost of the heat exchangers as a function of the conductance, $U A$. Equations 5.20-5.24 present the models of each component for a simple regenerative cycle (Carlson, Middleton and Ho, 2017; Neises and Turchi, 2019).

$$
\begin{gathered}
\text { Compressor cost }[\$]=6898 \cdot\left(\dot{W}_{c}[\mathrm{~kW}]\right)^{0.7865} \\
\text { Turbine cost }[\$]=7790 \cdot\left(\dot{W}_{t}[\mathrm{~kW}]\right)^{0.6842} \\
P H X \operatorname{cost}[\$]=3500 \cdot U A_{p h}[\mathrm{~kW} / \mathrm{K}] \\
\text { Regenerator cost }[\$]=1250 \cdot U A_{r e g}[\mathrm{~kW} / \mathrm{K}] \\
\text { Precooler cost }[\$]=2300 \cdot U A_{p c}[\mathrm{~kW} / \mathrm{K}]
\end{gathered}
$$

The cost of the primary heat exchanger (PHX) will be greater than the cost of the additional heat exchanger (AHX) due to the higher temperatures of the primary heat. The purpose of this work is not to predict the AHX cost, but to analyse how much it should cost in order to prefer multi-heating cycles over simple cycles. Therefore, the cost of the AHX will be expressed as a function of the PHX cost and a coefficient $\varphi$, with a value between 0 and 1 :

$$
A H X \operatorname{cost}[\$]=\varphi \cdot P H X \operatorname{cost}[\$]
$$




\subsubsection{Solar Field}

Previous analyses have focused mainly on the analysis of cycle performance. However, in a techno-economic analysis is fundamental to analyse the cost of the heat source. In the case of a CSP plant, this cost is represented by the Solar Field. The cost of the elements of a Solar Field with Solar Tower are expressed in Equations 5.26 and 5.27 (Ho et al., 2015).

$$
\begin{aligned}
& \text { Heliostats }_{p h} \operatorname{cost}[\$]=200 \cdot A_{p h}\left[\mathrm{~m}^{2}\right] \\
& \text { Receiver }_{p h} \operatorname{cost}[\$]=200 \cdot \dot{Q}_{p h}\left[\mathrm{~kW}_{\mathrm{t}}\right]
\end{aligned}
$$

In a multi-heating solar tower, the costs of these elements are expected to be lower in the additional-heat section. The cost of heliostats and receiver of this part will be expressed as a function of the costs of a Solar Tower by means of the coefficient $\varphi$ :

$$
\begin{gathered}
\text { Heliostats }_{a h} \text { cost }[\$]=\varphi \cdot \text { Heliostats }_{p h} \operatorname{cost}[\$] \\
\text { Receiver }_{a h} \operatorname{cost}[\$]=\varphi \cdot \text { Receiver }_{p h} \operatorname{cost}[\$]
\end{gathered}
$$

\subsubsection{CSP plant}

This chapter has focused on obtaining the optimal configuration of a CSP plant to maximize the system efficiency. The costs of each component will be calculated with the models presented in previous section. A tecno-economic analysis will help to identify the most critical components. However, the configuration with the maximum efficiency is not necessary the cheapest one. Therefore, the optimal configuration to minimize the cost will be also analysed.

All the parameters necessary to define a CSP plant have been already presented. Anyway, they will be summarized identifying the variables optimized in order to, on the one hand, ease a quick global overview, and on the other hand, make some comments about the values selected for the tecno-economic analysis. The parameters will be divided into two groups, power block and solar field.

\subsubsection{Power block}

The cycle parameters are shown in Table 5.2 divided into two columns: simple and multiheating. The variables to calculate the minimum system cost will be the low-side pressure and the additional-heat temperature. The effectiveness could also be optimized in order to include the regenerator size as variable. However, it has been seen that multi-heating cycles need high 
effectiveness to present advantages with respect to simple cycles. Therefore, the effectiveness is set to $\varepsilon=0.99$ as in previous calculations. In this way, the low-side pressure will define not only the size of the turbomachines, but also the size of the regenerator (lower pressure ratios involve bigger intermediate heat exchangers).

Table 5.2. Parameters of $\mathrm{CO}_{2}$ cycle in a CSP plant

\begin{tabular}{lll}
\hline Parameter & Simple & Multi-heating \\
\hline Maximum high-side pressure, $P_{H}$ & $25 \mathrm{MPa}$ & $25 \mathrm{MPa}$ \\
Minimum low-side pressure, $P_{L}$ & Optimized & Optimized \\
Compressor inlet temperature, $T_{c, \text { in }}$ & $50{ }^{\circ} \mathrm{C}$ & $50^{\circ} \mathrm{C}$ \\
Maximum additional-heat temperature, $T_{a h}$ & - & Optimized \\
Turbine inlet temperature, $T_{t, \text { in }}$ & $700{ }^{\circ} \mathrm{C}$ & $700{ }^{\circ} \mathrm{C}$ \\
Compressor efficiency, $\eta_{c}$ & 0.89 & 0.89 \\
Turbine efficiency, $\eta_{t}$ & 0.93 & 0.93 \\
Global effectiveness, $\varepsilon_{g l o b a l}$ & 0.99 & 0.99 \\
HTR effectiveness, $\varepsilon_{H T R}$ & - & 0.99 \\
Pressure drop in the primary heat exchanger, $\chi$ & 0.05 & 0.05 \\
Net power & $50 \mathrm{MWe}$ & $50 \mathrm{MWe}$ \\
\hline
\end{tabular}

The roadmap for CSP technology proposed by NREL (Mehos et al., 2017) suggests that the cycles must work under dry-cooled conditions in order to be able to operate in desert climates. Thus, the cycle in the system to analyse will work under with dry-cooled conditions, i.e., the compressor inlet temperature is set to $T_{c, i n}=50^{\circ} \mathrm{C}$. This temperature has been assumed for dry-cooled cycles in this work. However, this temperature will be established depending on the ambient temperature and the precooler features. In the literature, this temperature oscillates between $45^{\circ} \mathrm{C}$ (Neises and Turchi, 2019) and $55^{\circ} \mathrm{C}$ (Ho et al., 2015).

Chapter 4 analysed the features of the precooler and minimized the volume according to them. The resulting outlet temperatures of air in these heat exchangers were around $50{ }^{\circ} \mathrm{C}$. This temperature together with the inlet temperature $40{ }^{\circ} \mathrm{C}$, the $\mathrm{CO}_{2}$ temperatures and the type of heat exchanger (counter-flow) will determine the precooler conductance. 
Besides the precooler, the PHX and AHX are the other components needed in the power block with parameters external to the cycle. Table 5.3 shows all these parameters. The temperature difference between $\mathrm{CO}_{2}$ and $\mathrm{HTF}$ is needed to calculate the conductance of $\mathrm{PHX}$ and AHX.

Table 5.3. Parameters of precooler, primary heat exchanger and additional haet exchanger in a CSP system

\begin{tabular}{lc}
\hline Parameter & Value \\
\hline Air inlet temperature in precooler & $40{ }^{\circ} \mathrm{C}$ \\
Air outlet temperature in precooler & $50{ }^{\circ} \mathrm{C}$ \\
Temperature difference $\mathrm{CO}_{2}-\mathrm{HTF}$ in PHX & $25{ }^{\circ} \mathrm{C}$ \\
Temperature difference $\mathrm{CO}_{2}-\mathrm{HTF}$ in AHX & $25^{\circ} \mathrm{C}$ \\
\hline
\end{tabular}

\subsubsection{Solar field}

The solar field in a CSP plant is generally oversized in order to not only feed the cycle, but also store energy. The parameter in charge of defining this oversizing is the solar multiple. The solar multiple is the ratio between the maximum thermal power collected by the heat carrier fluid in the solar field and the thermal power required by the power block at nominal conditions. The sought amount of energy stored by the system will determine the required solar multiple (González-Portillo, Muñoz-Antón and Martínez-Val, 2017b). As the analysis of the energy stored is out of the purpose of this work, the tecno-economic analysis will consider a typical solar multiple $S M=2$.

The solar multiple and the rest of parameters of the solar field are shown in Table 5.4. The temperature of HTF in the additional-heat section will be variable, and it will be determined by the $\mathrm{CO}_{2}$ temperature of this heating with the temperature difference shown in Table 5.3. The concentration ratio of the additional-heat section will be set to the maximum possible value to get $500{ }^{\circ} \mathrm{C}$ in the surface of the receiver. In this way, the receiver achieves the maximum efficiency. Nonetheless, it has been seen that this concentration ratio can be highly reduced hardly affecting the efficiency. The rest of parameters have been already explained in previous sections. 
Table 5.4. Parameters of solar field in a CSP system

Parameter

Optical efficiency primary heat section

Geometrical concentration ratio primary heat section

Optical efficiency primary heat section

Geometrical concentration ratio primary heat section

Optical efficiency additional heat

Direct Normal Irradiance

Air temperature

Emissivity primary heat section

Emissivity additional heat section

Absorptivity

View factor

Heat transfer coefficient from receiver surface to air

Heat transfer coefficient from receiver surface to fluid

\section{Solar Tower}

\section{2}

0.6

0.6

1000

1000

0.6

0.6

Multi-Heating

Solar Tower

\section{2}

Optimized

0.7

$900 \mathrm{~W} / \mathrm{m}^{2}$

$900 \mathrm{~W} / \mathrm{m}^{2}$

$40{ }^{\circ} \mathrm{C}$

$40{ }^{\circ} \mathrm{C}$

0.85

0.85

0.15

0.95

0.95

1

1
$10 \mathrm{~W} / \mathrm{m}^{2} \mathrm{~K}$
$10 \mathrm{~W} / \mathrm{m}^{2} \mathrm{~K}$

$2000 \mathrm{~W} / \mathrm{m}^{2} \mathrm{~K}$

The cost estimation of the additional-heat components does not directly depend on the concentration ratio. However, the coefficient $\varphi$ will indirectly represent it: higher concentration ratios involve more complex systems, and therefore more expensive. In this analysis, concentration ratio of the additional-heat section is set to the maximum possible value to get $500{ }^{\circ} \mathrm{C}$ in the surface of the receiver. However, the selection of the optimum concentration ratio for the additional-heat section will depend on the design of this part of the solar field.

\subsubsection{Tecno-economic analysis}

The first calculation of costs will be made for the CSP configuration obtained in previous section to maximize the system efficiency. The pressure ratio in the system with simple cycle 
was $r=3.22$ and the the pressure ratio in the system with multi-heating cycle was $r=3.01$. The costs of the components of power block in these two systems are shwon in Figure 5.25. The cost of the power blowck with multi-haeting is calculated with $\varphi=1$ (the costs of the additional-heat section is the same than the costs of the primary-heat section) and $\varphi=0.5$ (the costs of the additional-heat section are half the costs of the primary-heat section).

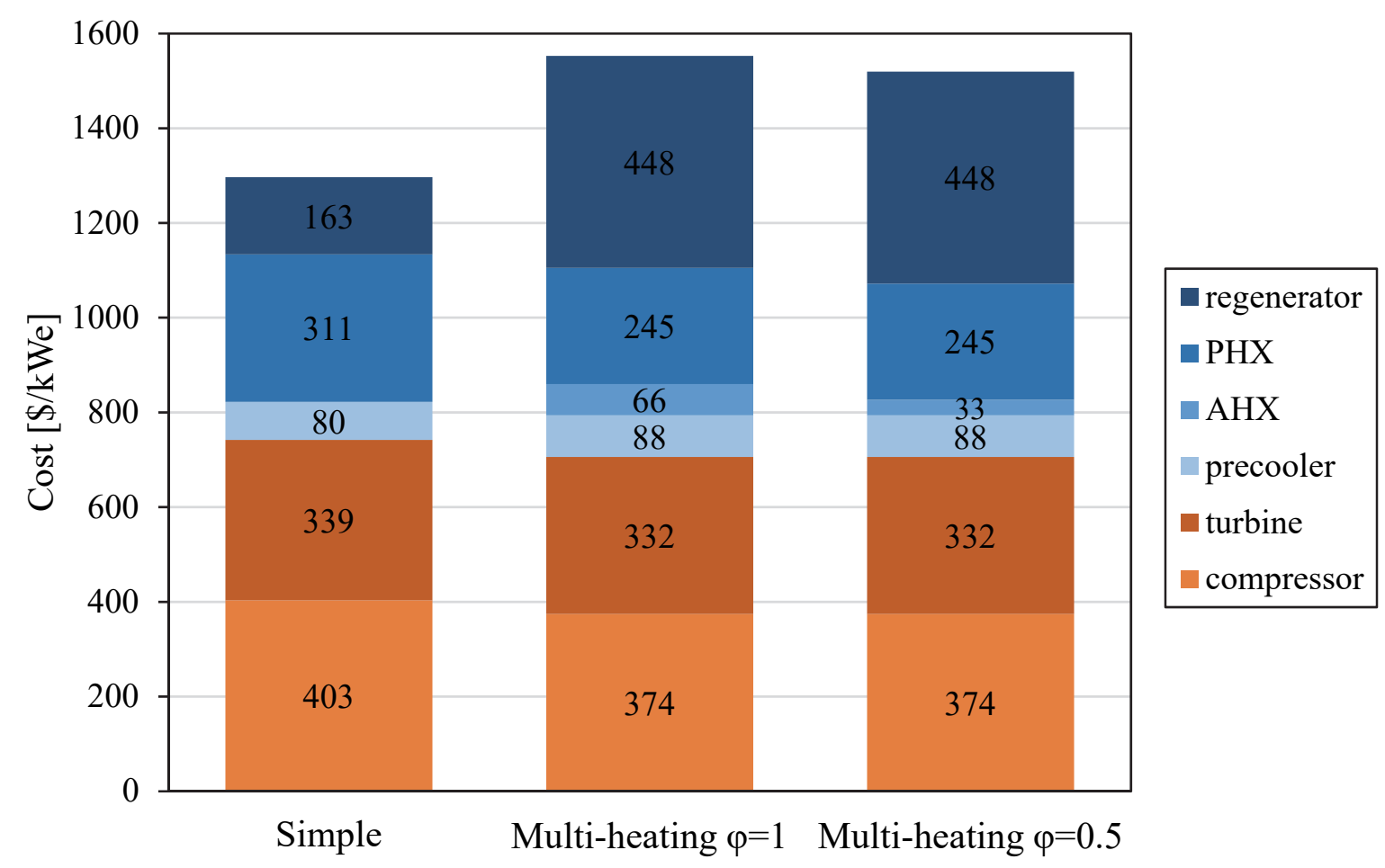

Figure 5.25. Cost of the power block in $\$ / \mathrm{kW}_{\mathrm{e}}$ depending on the type of cycle (simple or multi-heating) and the costs of the additional-heat section (determined by $\varphi$ )

The main difference between the costs in simple and multi-heating cycles is the cost of the regenerator. This cost is almost three times bigger in multi-heating cycles, which results in a greater cost of the power block for these cycles. The cost of the turbomachines is slightly greater in the simple cycle due to the greater pressure ratio. However, the difference barely affects the total cost of the power block.

The cost of the heat exchangers $\mathrm{CO}_{2}-\mathrm{HTF}$, PHX and AHX, is $311 \$ / \mathrm{kW}$ in the simple cycle and the same in the multi-heating cycle with $\varphi=1$. It is reduced to $278 \$ / \mathrm{kW}_{\mathrm{e}}$ when the cost of the additional heat section is reduced by $\varphi=0.5$. Nonetheless, this reduction barely affects the total cost of the power block. The result is that, with these configurations, the simple cycle is $223 \$ / \mathrm{kW}_{\mathrm{e}}$ cheaper than the cheapest version of multi-heating.

Figure 5.26 shows the cost of the solar field components heliostats and receiver. The cost of the Solar Tower receiver is $890 \$ / \mathrm{kW}$, the same than the cost of the multi-heating solar tower 
receiver with $\varphi=1$. If the cost of the additional-heat section is reduced by $\varphi=0.5$ then the total cost of the receiver would be $95 \$ / \mathrm{kW}_{\mathrm{e}}$ cheaper. The higher efficiency of the multiheating solar tower implies a smaller area of heliostats than in the case of the standard solar tower. Therefore, the cost of the heliostats when there is multi-heating is lower: $94 \$ / \mathrm{kW}$ e lower if $\varphi=1$ and $256 \$ / \mathrm{kW}$ lower if $\varphi=0.5$.

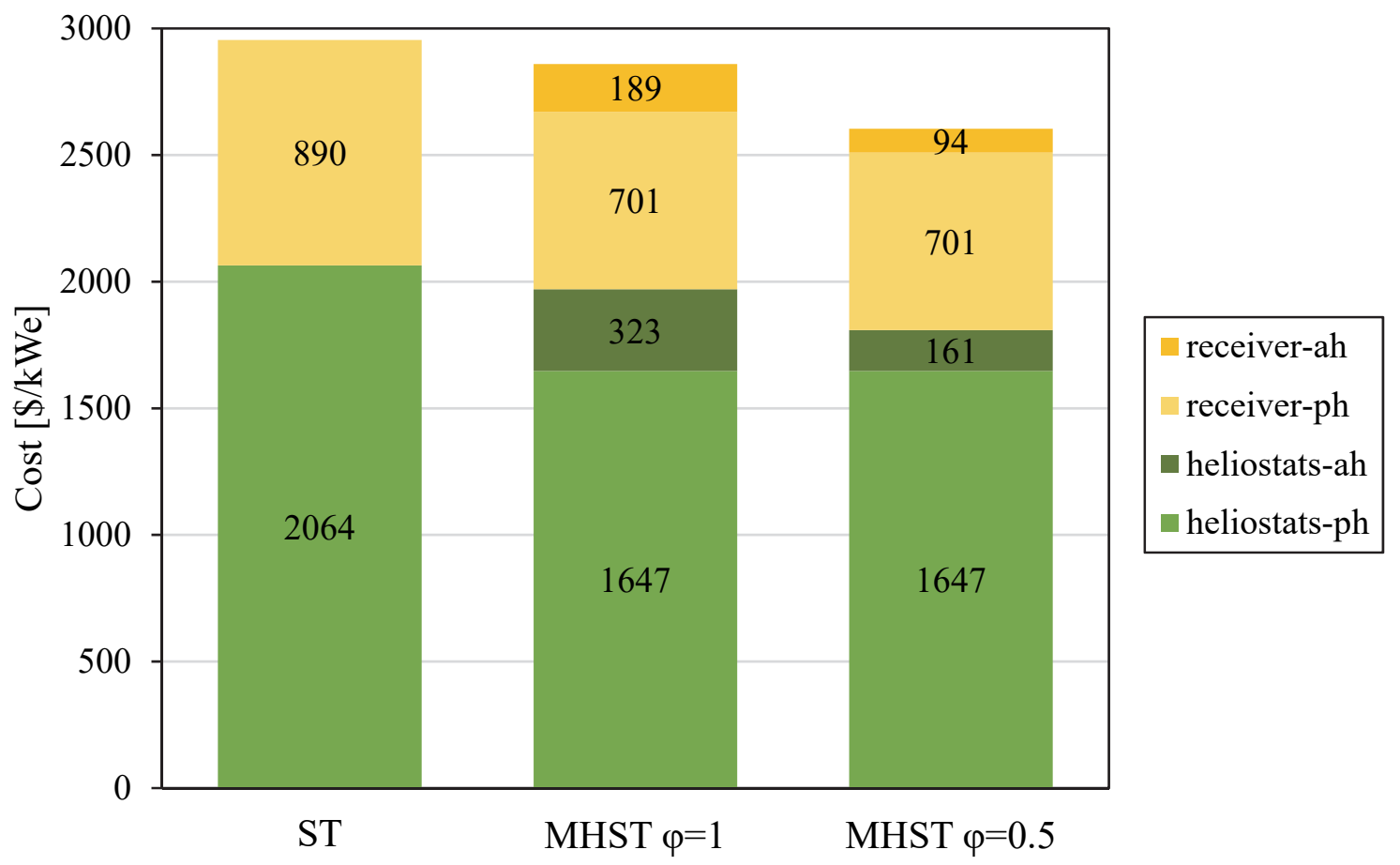

Figure 5.26. Cost of the solar field in $\$ / \mathrm{kW}_{\mathrm{e}}$ depending on the type of receiver (solar tower,

ST, and multi-heating solar tower, MHST) and the costs of the additional-heat section (determined by $\varphi$ )

The sum of the costs of Figure 5.25 and Figure 5.26 is represented in Figure 5.27. The cost of the power block is lower in the $\mathrm{SC}+\mathrm{ST}$ system due to the smaller regenerator. However, the cost of the solar field is lower in the systems with multi-heating $(\mathrm{MH})$, especially in the case of additional-heat section with $\varphi=0.5$. The result is that the total cost of the SC+ST system is $4251 \$ / \mathrm{kW}_{\mathrm{e}}$, and the cost of the MHC+MHST system is $3.8 \%$ higher if $\varphi=1$ and $4 \%$ lower if $\varphi=0.5$. The costs of SC+ST and MHC+MHST systems are very similar, and the additionalheat section will decide which is the cheapest system.

Figure 5.27 shows the costs of systems optimized to maximize the efficiency. However, the configuration (and therefore the cost) of the system optimized to minimize its cost will be different. It is important to consider both options to understand the reasons of the optimum configurations and identify the most critical parts of each system. 


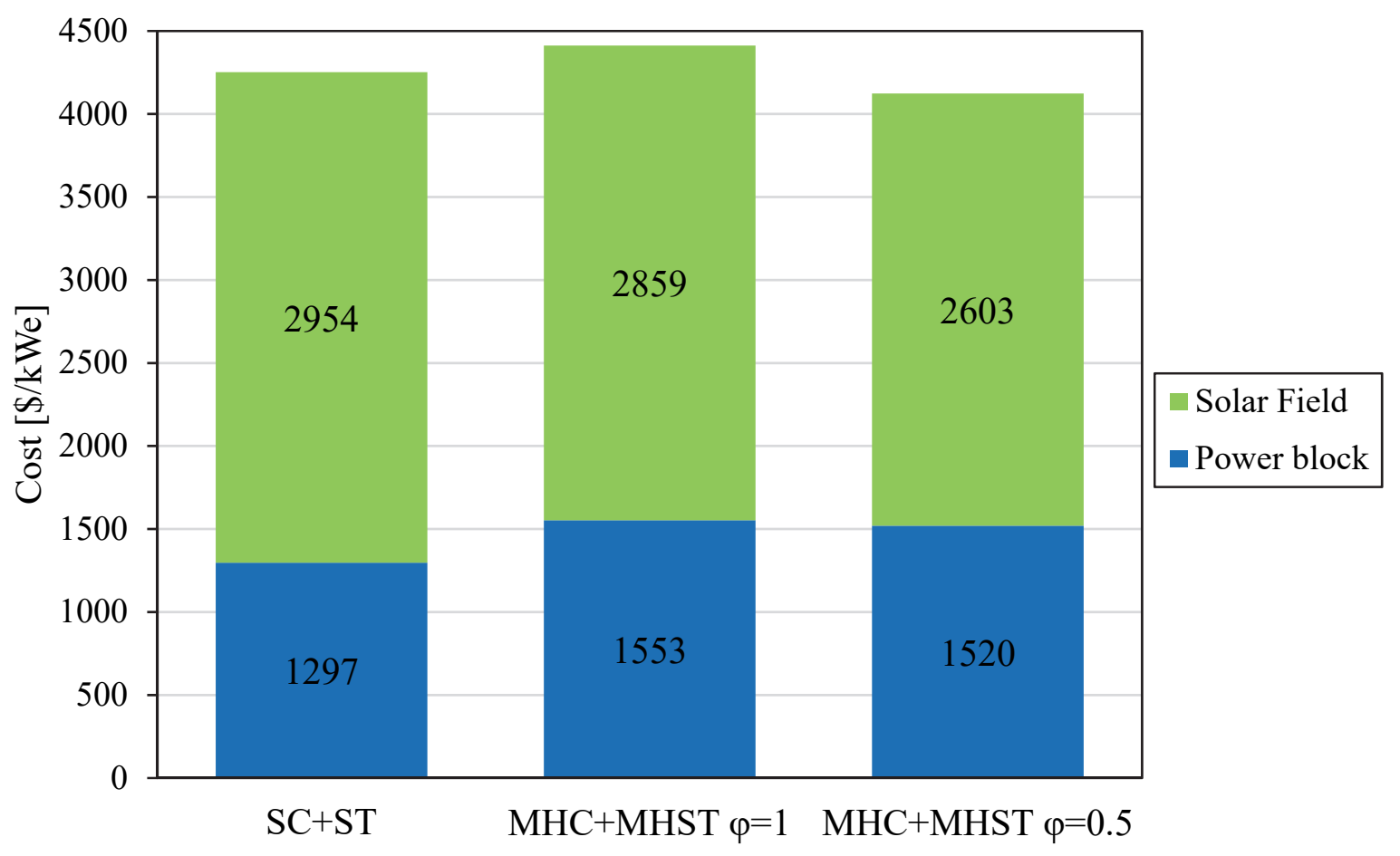

Figure 5.27. Cost of the CSP plant in $\$ / \mathrm{kW}_{\mathrm{e}}$ when the system efficiency is maximized.

Table 5.5 shows the optimized parameters when the configuration maximizes the system efficiency (eff) and when the configuration minimizes the system cost (cost), and Figure 5.28 compares the resulting costs. Although all the configurations obtained to minimize the cost get to reduce the cost, the changes in the configuration are different for each type of system.

Table 5.5. System configuration of the CSP systems depending on its optimization

\begin{tabular}{|c|c|c|c|c|c|c|}
\hline \multirow[b]{2}{*}{ Parameter } & \multicolumn{2}{|c|}{$\mathbf{S C}+\mathbf{S T}$} & \multicolumn{2}{|c|}{ MHC + MHST $\varphi=1$} & \multicolumn{2}{|c|}{ MHC + MHST $\varphi=0.5$} \\
\hline & eff & cost & eff & $\operatorname{cost}$ & eff & cost \\
\hline Pressure ratio, $r$ & 3.22 & 2.74 & 3.01 & 2.74 & 3.01 & 2.5 \\
\hline Additional-heat temperature, $T_{a h}$ & - & - & $322{ }^{\circ} \mathrm{C}$ & - & $322{ }^{\circ} \mathrm{C}$ & $269^{\circ} \mathrm{C}$ \\
\hline
\end{tabular}

The cost of the SC+ST system decreases from $4251 \$ / \mathrm{kW}_{\mathrm{e}}$ to $4211 \$ / \mathrm{kW}$ e when the cost is minimized. The system had a pressure ratio $r=3.22$ and the new pressure ratio is $r=2.74$, which involves a lower cost in the turbomachines. The rest of the components increase its size, and therefore, its cost. Nonetheless, the cost reduction in the turbomachines compensates the cost increase in the rest of components. The result is that the configuration optimized to minimize the cost is $40 \$ / \mathrm{kW}$ e cheaper. 


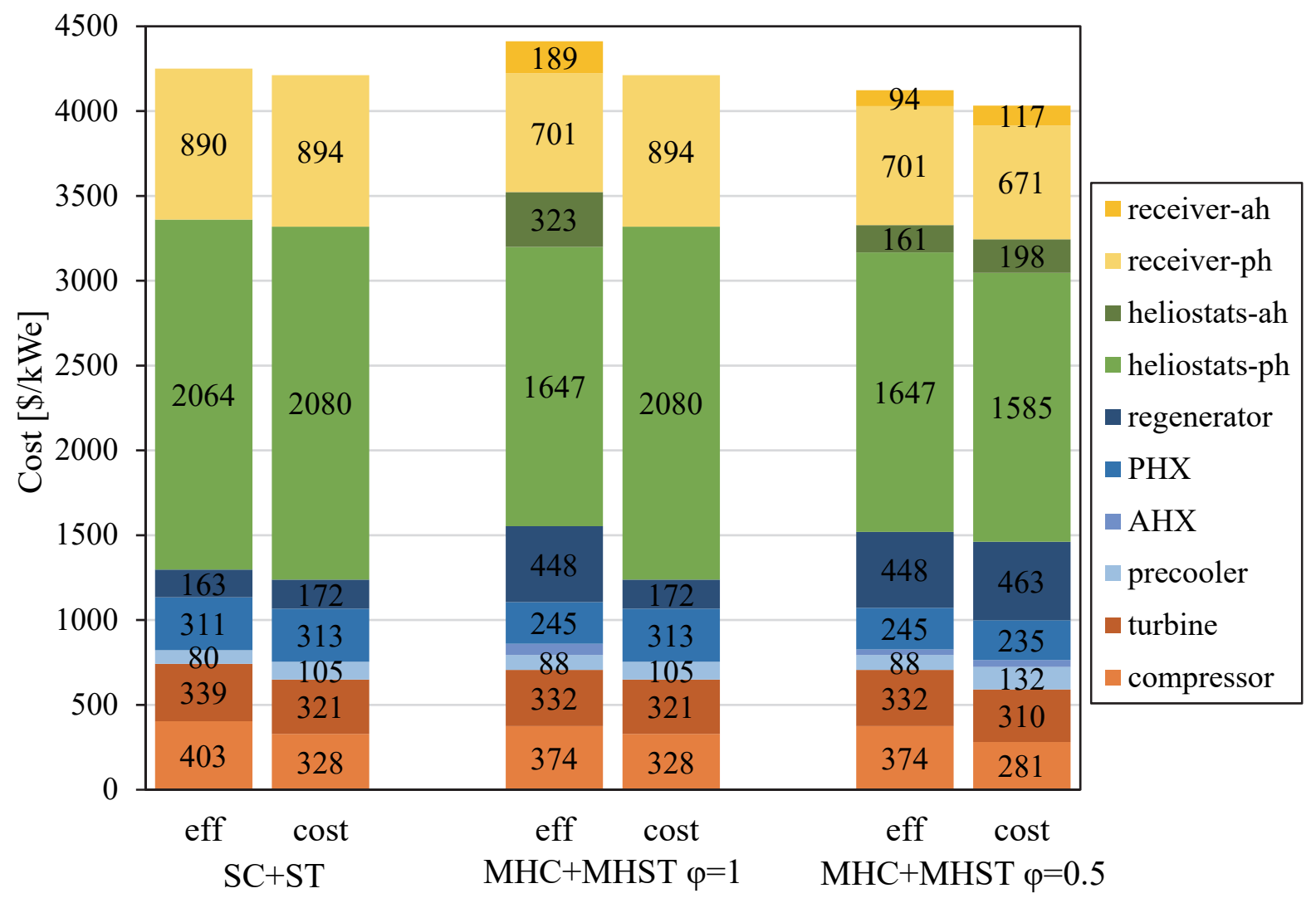

Figure 5.28. Cost of the CSP plant in $\$ / \mathrm{kW}_{\mathrm{e}}$ when the system efficiency is maximized (eff) and when the system cost is minimized (cost)

The optimized configuration to minimize the cost of the MHC+MHST systems is different depending on the cost of the additional-heat section (represented by $\varphi$ ). In the case of the cost represented by $\varphi=1$, the main difference is that the optimum configuration when the cost is minimized has no additional heat, i.e., it is a $\mathrm{SC}+\mathrm{ST}$ system. The reason is the high cost of the regenerator when an additional heat is supplied. In this way, the regenerator in the case of maximizing the efficiency (configuration with additional heat) costs $448 \$ / \mathrm{kW}$ and it is reduced to $172 \$ / \mathrm{kW}_{\mathrm{e}}$ when the system cost is minimized (configuration without additional heat).

In the case of the additional-heat cost represented by $\varphi=0.5$, the optimum configuration integrates multi-heating in both cases, when the efficiency is maximized and when the cost is minimized. The optimum configuration of the latter case has a pressure ratio $r=2.5$, considerably smaller than the pressure ratio in the case of maximizing the efficiency, $r=3.01$. The lower pressure ratio involves that the irreversibilities in the regenerator are larger, and therefore, the additional heat can represent a bigger part of the total thermal power supplied to the cycle. The result is that the configuration obtained to minimize the cost achieves a lower cost by reducing the turbomachine size (and so its cost) and increasing the additional heat (but 
not necessarily its cost because the additional-heat section is cheaper). The additional-heat power represents a $21 \%$ of the total in the case of maximizing the efficiency and a $26 \%$ in the case of minimizing the cost.

In summary, if the costs of the additional-heat section are the same than the costs of the primary-heat section then the best configuration of plant is the Solar Tower coupled to a simple regenerative cycle. However, if the cost of the additional-heat section is half, then the optimum configuration is the multi-heating solar tower coupled to multi-heating cycle. Anyway, the costs are not very different: $4211 \$ / \mathrm{kW}_{\mathrm{e}}$ in the system with Solar Tower and $4031 \$ / \mathrm{kW}_{\mathrm{e}}$ in the system with multi-heating solar tower and $\varphi=0.5$.

A reduction of $50 \%$ in the cost may seem excessive. However, the cost of concentrator and receiver highly depends on concentration and temperature, and reducing these two variables may highly reduce the receiver cost. For example, in the software SAM (NREL, 2017), the estimated cost for a solar field with solar tower is $460 \$ / \mathrm{kW}_{\mathrm{t}}$, but if the solar field is PTC (with lower concentration ratios and lower temperatures) the cost is reduced to $350 \$ / \mathrm{kW}_{\mathrm{t}}$. Further cost reductions can be found in simpler concentrators such as Linear Fresnel Collectors. Sait et al (2015) estimate that the cost of these collectors can be $2 / 3$ of the cost of PTC, which means a $50 \%$ of the cost of a solar field with solar tower. A good design of the pair concentrator-receiver has the potential to greatly reduce the costs of the additional-heat section.

Even if $\varphi=0.5$ still seems too optimistic, the main purpose of this value has been to show the potential of multi-heating, and not setting it as the real cost of the additional-heat section. Other values of $\varphi$ will lead to different results. Figure 5.29 shows the parameters of the optimum configurations to minimize the system cost as a function of $\varphi$, and Figure 5.30 shows the resulting system costs. The value $\varphi=1$ means that the costs of primary-heat and additional-heat sections are the same, and $\varphi=0$ that the cost of the additional-heat section is null.

When the parameter $\varphi$ is greater than 0.9 , the optimum configuration is a simple cycle coupled to a solar tower. The pressure ratio is $r=2.74$ and the additional-heat tempearture, $T_{a h}$, is the compressor outlet temperature $T_{c, \text { out }}=124{ }^{\circ} \mathrm{C}$, i.e., there is no additional heat. Decreasing the parameter $\varphi$ below 0.9 involves an increase in the additional-heat temperature, i.e., the multi-heating starts to be the optimum configuration. The additional-heat temperature increases and the pressure ratio decreases. Therefore, the additional heat represents a larger portion of the total thermal power supplied when the parameter $\varphi$ decreases. 


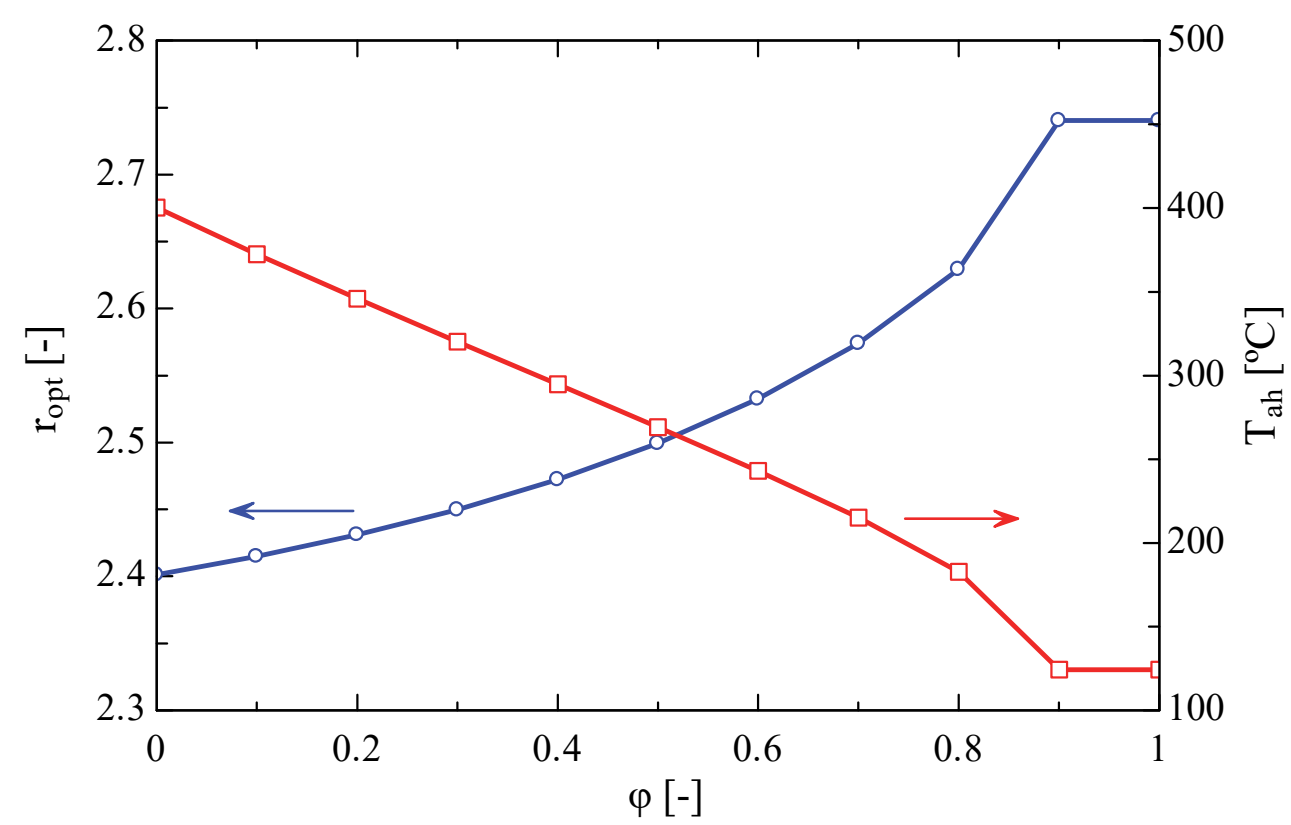

Figure 5.29. Optimum pressure ratio, $r_{o p t}$, and additional-heat tempearture, $T_{a h}$, as a function of the parameter $\varphi$ accounting for the cost of the additional-heat section

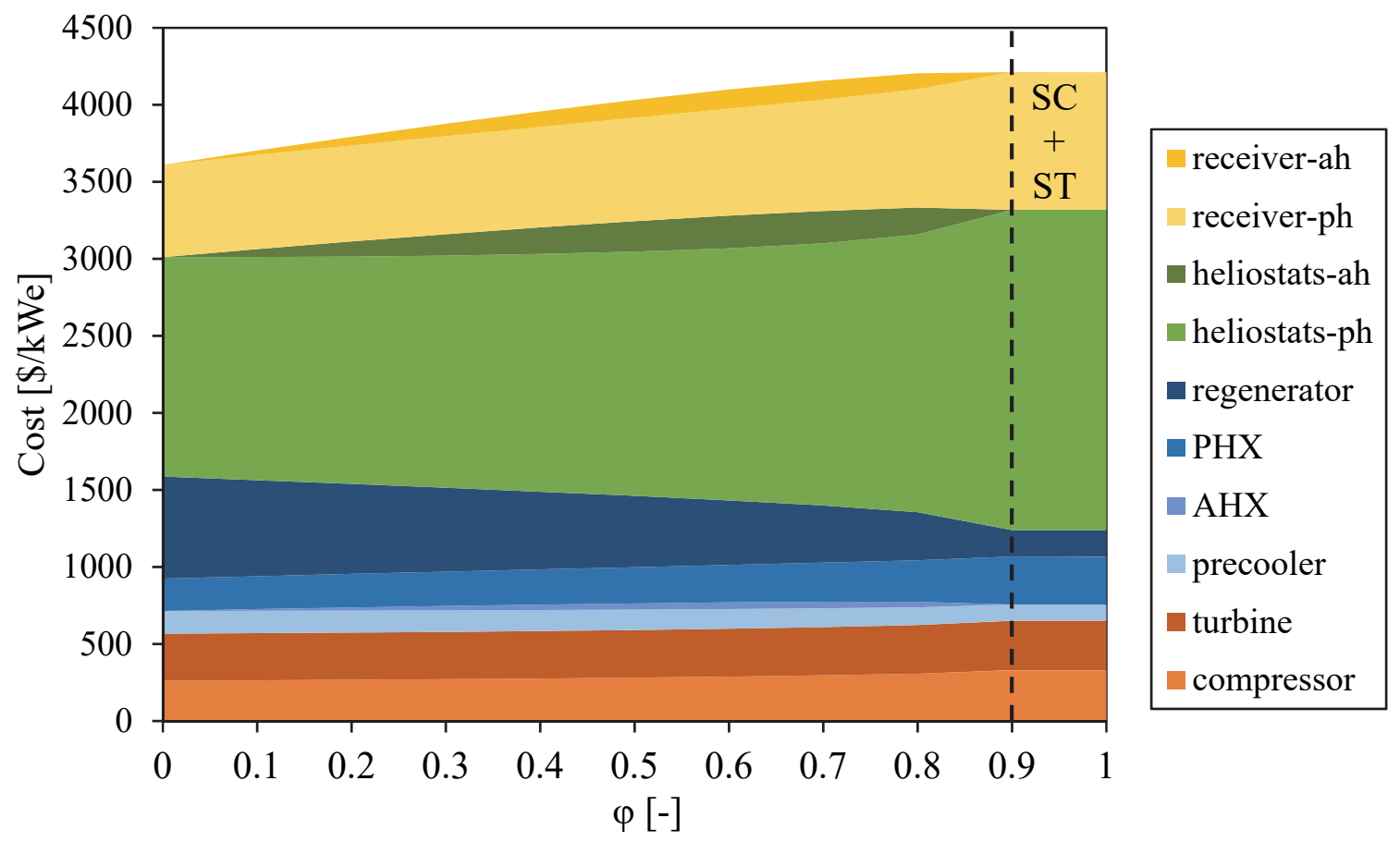

Figure 5.30. System cost in $\$ / \mathrm{kW}_{\mathrm{e}}$ as a function of the parameter $\varphi$ accounting for the costs of the additional-heat section

The resulting costs of each component are shown in Figure 5.30. The larger proportion of additional heat at lower $\varphi$ can be noticed in the higher cost of the regenerator as larger intermediate heat exchangers are needed to integrate larger additional heats. While the cost of the regenerator increases at low $\varphi$, the total cost of solar field (receiver and heliostats) 
decreases due to the lower cost of the additional-heat section. The rest of the components vary its cost, but its impact in the total system cost is neglectable. The result is that the cost of the system when $\varphi \geq 0.9$ is the cost of the SC+ST system: $4211 \$ / \mathrm{kW}_{\mathrm{e}}$. This cost decreases when the additional-heat section gets cheaper. The cheapest system would be obtained if the additional-heat section were free $(\varphi=0): 3610 \$ / \mathrm{kW}$.

The system composed of multi-heating solar tower and multi-heating cycle will be cheaper when the costs of the additional-heat components are, at least, $10 \%$ lower than the costs of the primary-heat components. However, in order to choose the system with multi-heating solar tower, the cost reduction with respect to the system with solar tower should be substantial for two reasons. The first one is that the system with multi-heating is more complex and may involve extra costs associated to operation and maintenance, failures... And the second one is that due to the uncertainty of the component costs, if the difference between the system costs is small, it would be a conservative solution to choose the simplest alternative.

\subsection{Summary}

This Chapter studies the integration of simple and multi-heating cycles in CSP systems. The solar field in charge of supplying the thermal power to these systems is different depending on the cycle features. A Solar Tower works at high concentrations and high temperatures achieving high efficiencies and fitting perfectly to simple cycles. These high temperatures and concentrations also match the primary-heat requirements of multi-heating cycles, but not those of the additional heat. Thus, another section is added to the receiver with different conditions of temperature and concentration. The result is a solar tower-like receiver called multi-heating solar tower.

The multi-heating solar tower is divided into two sections. The primary-heat section has similar conditions than the receiver in a Solar Tower, and the additional-heat section accommodates the concentration ratio to the low temperatures of the additional-heat fluid. The result is a receiver with higher efficiency than a Solar Tower and lower thermal requirements. The higher efficiency is due to the combination of three features in the additional-heat section: low temperature, low emissivity (thanks to its low concentration ratio) and high optical efficiency.

Two systems are compared: simple cycles coupled to solar towers and multi-heating cycles coupled to multi-heating solar towers. The latter has higher efficiency in all the cases, 
especially at high turbine inlet temperatures and low concentration ratios. For the reference case, with a turbine inlet temperature $T_{t, i n}=700{ }^{\circ} \mathrm{C}$ and concentration ratio in the primary-heat section $C_{\text {opt }}=600$, the efficiency of a CSP plant with multi-heating is up to $6.1 \%$ higher under wet-cooled conditions and up to $4.7 \%$ under dry-cooled conditions. This higher efficiency involves a smaller area of concentrators needed, which represents a large part of the total system cost.

An estimation of costs shows that the optimum configuration highly depends on the cost of the intermediate heat exchangers and the cost of the additional-heat components. If the cost of the additional-heat components is similar to the cost of the primary-heat components, then the best plant configuration is a solar tower coupled to a simple regenerative cycle, and the cost is $4211 \$ / \mathrm{kW}_{\mathrm{e}}$. However, if the cost of the additional-heat components is below $90 \%$ of the cost of the primary-heat components, the best configuration is a multi-heating solar tower coupled to a multi-heating cycle. For example, if the cost of the additional-heat components were half the cost of the primary-heat components, the cost of the system with multi-heating would be $4031 \$ / \mathrm{kW}_{\mathrm{e}}$, i.e., $4.5 \%$ cheaper than the system with solar tower and simple cycle. These costs are obtained for dry-cooled conditions, which will represent the majority of the CSP plants (Mehos et al., 2017). However, if the plant were in a place with easy access to water, the cost would be lower and the benefit of multi-heating greater.

This Chapter is the end of a pathway that has merged Thermodynamics and Heat Management. As a result, a new type of CSP plant has been proposed by using the basis of thermal coherence to obtain higher efficiencies with lower material requirements. The new system shows a great potential to reduce the cost of CSP plants by adding multi-heating to the receiver and to the cycle. 


\section{CONCLUSIONS}

This work shows the potential of joining thermodynamics and heat management under the same analysis. A novel CSP plant layout has been designed as a result of this coherent integration. In this layout, the specific features of pericritical multi-heating cycles perfectly match the solar thermal power characteristics of the multi-heating solar tower. The new design has higher performance and potential lower costs than standard CSP plant configurations.

The analysis towards the final layout has traveled a pathway that has produced several interesting results. This chapter summarizes these outputs classifying them in three groups: pericritical cycles, multi-heating cycles and CSP.

\subsection{Pericritical cycles}

Regenerative pericritical cycles have been studied by means of a comprehensive and systematic analysis. The cycles are defined by means of reduced thermophysical properties with the purpose of generalizing the characterization of these cycles setting aside the fluid. All the cycles have the same Carnot efficiency to be more equitable in the comparison. The analysis characterizes the cycle performance depending on the compressor position in the thermodynamic region around the critical point.

The analysis of the pericritical region shows the special relevance of the discontinuity line, defined as the maximum logarithmic factor of isobaric expansion in each isobar. This line separates the pericritical region into two: one with liquid features and the other one with gas 
features. These two regions exist even at pressures above the critical pressure. The transition from one region to the other is more abrupt at low pressures and softer at high pressures.

For the same pressure ratio, pericritical cycles with the compressor working in the liquid region require lower compression power than cycles with the compressor in the gas region. However, the lower compression power involves larger irreversibilities during the heat regeneration, which results in greater thermal power supply. The balance between compression work and the limitations in the heat regeneration phase characterizes the thermal efficiency. The convenience of reducing one or the other to maximize the efficiency depends on the parameters defining the cycle.

The characterization of the pericritical region allows to study pericritical cycles without any constraint, which leads the analysis to places where previous investigations have not reached due to own imposed constraints. The best example are the previous investigations focused in specific regions of the pericritical region, such as supercritical cycles. Most of these investigations consider that the compressor inlet temperature must be above the critical temperature in order to avoid phase change (Dostal, 2004). However, the analysis of the pericritical region shows that if the low-side pressure of the cycle is above the discontinuity line the fluid goes through the liquid region to the gas region (or vice versa) regardless of the compressor inlet temperature. Therefore, the constraint of the compressor inlet temperature seems to make no sense. The limitation of this temperature will be imposed by the heat sink and technoeconomic features.

The characterization of the pericritical region allows to develop an optimization scheme for pericritical cycles. The low-side pressure is optimized to maximize the thermal efficiency for different compressor inlet temperatures and high-side pressures. The best suited operating conditions of pericritical cycles with common inefficiencies in its components (turbomachines efficiency $\eta_{t} \approx 0.85$ and heat exchanger effectiveness $\varepsilon \approx 0.95$ ) are analysed. When the highside pressure is close to or below the pressure corresponding to the compressor inlet temperature in the discontinuity line, the optimum compressor position is the ideal gas region, where the thermal efficiency is the same regardless of the compressor inlet temperature and the high-side pressure. However, if the high-side pressure is well above the discontinuity line, the thermal efficiency increases when the high-side pressure does it. This occurs because the optimum compressor position is closer to the liquid region or inside it, and the smaller compression work compensates the regeneration irreversibilities. For a specific high-side 
pressure well above the discontinuity line, cycles with lower compressor inlet temperature can achieve higher efficiencies.

These conditions are found for the common inefficiencies $\eta_{t} \approx 0.85$ and $\varepsilon \approx 0.95$. However, these inefficiencies may have a great impact on the thermal efficiency and on the optimum pressure ratio needed to maximize it. Cycles with low regenerator effectiveness and high turbomachines efficiency achieve its maximum performance at large pressure ratios with the compressor working in the gas-like region, and cycles with high regenerator effectiveness and low turbomachines efficiency achieve its maximum performance at smaller pressure ratios with the low-side pressure in the discontinuity line or close to it.

\subsection{Multi-heating cycles}

The thermodynamic analysis of pericritical cycles shows a great mismatch between the isobaric specific heats of the high- and low-pressure sides along the regeneration phase. This mismatch limits the cycle performance. The proposed method to reduce this problem is to supply additional heats at low temperature along the regeneration, which substitute part of the heat supplied by the main source. The result is that the exergetic cost of the cycles decreases, and the exergy efficiency increases with respect to simple regenerative cycles. The novel cycle layout is called multi-heating cycle.

Multi-heating cycles deal with the heat exchange during the regeneration and with the heat supplied by additional heat sources. The cost of the heat sources is considered by means of an exergetic analysis. In this way, the relation between cost and temperature characteristic from CSP is introduced in the analysis. Anyway, the multi-heating cycle could integrate other heat sources, and they would improve the system efficiency if the cost of the heat supply is lower as the temperature decreases. This opens the possibility of hybridizing heat sources from different nature.

Multi-heating cycles can contain several additional heat sources. However, the main analysis focuses on the simplest case with one additional heat as it presents the bigger potential. The analysis of these multi-heating cycles is guided with the help of the discontinuity line. In order to increase the exergy efficiency more than $10 \%$ with respect to simple cycles, the high-pressure side must be above two times the pressure corresponding the compressor inlet temperature in the discontinuity line. The compressor position in the thermodynamic diagram determines the increase of exergy efficiency with respect to simple regenerative cycles. This 
increase may be up to $24 \%$ if the reduced compressor inlet temperature is $T_{c, i n, r}=0.9$ and the reduced high-side pressure is around $P_{H, r}=3$. These values are calculated for the common inefficiencies $\eta_{t} \approx 0.85$ and $\varepsilon \approx 0.95$. Multi-heating cycles with lower turbomachines efficiencies and higher heat exchanger effectiveness achieve larger increases of exergy efficiency.

The benefit of multi-heating with respect to simple regenerative cycles increases by lowering the reduced compressor inlet temperature. The minimum achievable reduced compressor inlet temperature depends on the critical temperature and the heat sink temperature. After a general thermodynamic analysis of simple and multi-heating cycles using reduced temperatures and pressures, the analysis focuses on cycles with $\mathrm{CO}_{2}$. In this case, the reduced compressor inlet temperatures analysed are 0.997 and 1.063 , corresponding to $30{ }^{\circ} \mathrm{C}$ and $50{ }^{\circ} \mathrm{C}$, for wet- and dry-cooled cycles, respectively. While in the generic analysis all the cycles had the same Carnot factor, in the analysis of $\mathrm{CO}_{2}$ cycles the turbine inlet temperature is set to a constant value $\left(700^{\circ} \mathrm{C}\right.$ as reference value). This means that the Carnot efficiency of wet-cooled cycles is higher. Thus, dry-cooled cycles will achieve lower efficiencies, but they may be necessary in locations where the access to water is limited.

The high compressor and turbine efficiencies in pericritical $\mathrm{CO}_{2}$ cycles involve that the effectiveness must be high to fully exploit multi-heating cycles. The problem is that increasing the effectiveness involves bigger intermediate heat exchangers. The effect of the intermediate heat exchanger size in these cycles is analysed. Three different ways to measure the influence of heat exchangers are considered: effectiveness, conductance and volume.

The effectiveness method is very useful to understand the potential of the cycle. This is the reason why it has been used during the generic thermodynamic analysis. However, the optimum cycle configuration with high effectiveness values may involve very small pressure ratios, and therefore too big heat exchangers. Thus, it is important to evaluate the thermal conductance or the volume of the intermediate heat exchangers. The type of heat exchanger used to estimate the volume is the Printed Circuit Heat Exchanger (PCHE).

The exergy efficiency grows in simple and multi-heating cycles when the intermediate heat exchangers get bigger. This growth tends to a horizontal asymptote when the effectiveness is around $\varepsilon=0.99$. The maximum benefit of multi-heating in terms of efficiency occurs at this effectiveness. However, the higher exergy efficiency of multi-heating cycles comes at the expense of bigger intermediate heat exchangers. Table 6.1 compares heat exchanger volume and exergy efficiency of the configurations analysed when the global effectiveness is $\varepsilon=0.99$. 
The rotor diameters of the turbomachines are also shown with the only purpose of highlight its small size in comparison to the heat exchangers.

Table 6.1. Cycle configuration with global effectiveness $\varepsilon=0.99$

\begin{tabular}{lcccc}
\hline \multirow{2}{*}{ Parameter } & \multicolumn{2}{c}{ Wet-cooling } & \multicolumn{2}{c}{ Dry-cooling } \\
\hline Regenerator volume & Simple & Multi-heating & Simple & Multi-heating \\
Precooler volume & $5.1 \mathrm{~m}^{3}$ & $9.4 \mathrm{~m}^{3}$ & $9.6 \mathrm{~m}^{3}$ & $16.3 \mathrm{~m}^{3}$ \\
Compressor rotor diameter & $1 \mathrm{~m}^{3}$ & $1.8 \mathrm{~m}^{3}$ & $36 \mathrm{~m}^{3}$ & $44.1 \mathrm{~m}^{3}$ \\
Turbine rotor diameter & $0.28 \mathrm{~m}$ & $0.28 \mathrm{~m}$ & $0.45 \mathrm{~m}$ & $0.4 \mathrm{~m}$ \\
Exergy efficiency & $0.55 \mathrm{~m}$ & $0.55 \mathrm{~m}$ & $0.61 \mathrm{~m}$ & $0.65 \mathrm{~m}$ \\
\hline
\end{tabular}

Wet-cooled cycles achieve higher efficiencies with smaller regenerators than dry-cooled cycles. However, the biggest difference between wet- and dry-cooled cycles is the precooler size. It represents a small part of the total heat exchangers volume in wet-cooled cycles, and a big part of dry-cooled cycles. The volume of heat exchangers is much smaller in the case of wet-cooled cycles. Therefore, this option will certainly be the best option in case of water availability in the plant. However, the lack of water could involve a preference for dry-cooled cycles in some type of plants, such as CSP plants.

\subsection{CSP}

The last part of this work is the integration of multi-heating cycles in CSP plants. The characteristics of CSP plants are analysed with the purpose of matching them to the multiheating requirements. A new type of solar field is created with this purpose: the multi-heating solar tower.

The configuration of the multi-heating solar tower is similar to the configuration of a solar tower, but with receiver and heliostats divided into two sections to accommodate the impinging solar flux to the multi-heating requirements. Each section meets different requirements in concentration ratio, fluid temperature, and absorbed heat flux. The primaryheat section has similar requirements than the receiver in a solar tower: high concentration ratio and high temperature. The additional-heat section accommodates the concentration ratio 
to the low temperatures required by the additional heat in multi-heating cycles. The efficiency of multi-heating solar towers is higher than the efficiency of standard solar towers due to the additional-heat section, which has low temperature, low emissivity and high optical efficiency.

Two systems have been compared at different conditions: simple cycles coupled to solar towers and multi-heating cycles coupled to multi-heating solar towers. In the first place, these systems have been optimized to obtain the maximum efficiency. For the case with a turbine inlet temperature $T_{t, i n}=700{ }^{\circ} \mathrm{C}$ and geometrical concentration ratio in the primary-heat section $C_{\text {geo }}=1000$, the efficiency of the optimum CSP plant with multi-heating dry-cooled cycle and multi-heating solar tower is $4.7 \%$ higher than the efficiency of the optimum CSP plant with simple cycle.

A techno-economic analysis shows that the optimum configuration to minimize the cost of a CSP plant with multi-heating highly depends on the cost of the additional-heat components (including concentrators). If the cost of the additional-heat section components is similar to the cost of the primary-heat section components, then the plant with multi-heating is more expensive than the plant with simple cycle. However, if the cost of the additional-heat section components is below $90 \%$ of the cost of the primary-heat components, the best configuration is the plant with multi-heating.

The section of the additional heat has a great potential to reduce costs due to its softer requirements in temperature and concentration ratio. The electricity cost of CSP plants with multi-heating has been analyzed as a function of the cost of the additional-heat components. Figure 6.1 shows the cost of a plant with simple cycle and the other with multi-heating assuming that the cost of the additional-heat section components were half the cost of the primary-heat section components. The cost of the plant with multi-heating is $4.3 \%$ lower. The optimum configuration of the plant with multi-heating contains a large intermediate heat exchanger to increase the additional heat. The result is a more expensive intermediate heat exchanger, but a cheaper solar field. The benefit of multi-heating will highly depend on the cost of the intermediate heat exchangers and the additional-heat components.

These costs have obtained for dry-cooled conditions, which will represent the majority of the CSP plants (Mehos et al., 2017). However, if the plant were in a place with easy access to water, the benefit of the plant with multi-heating would be higher, and the cost of the power block (and so the cost of the CSP system) would be lower. 


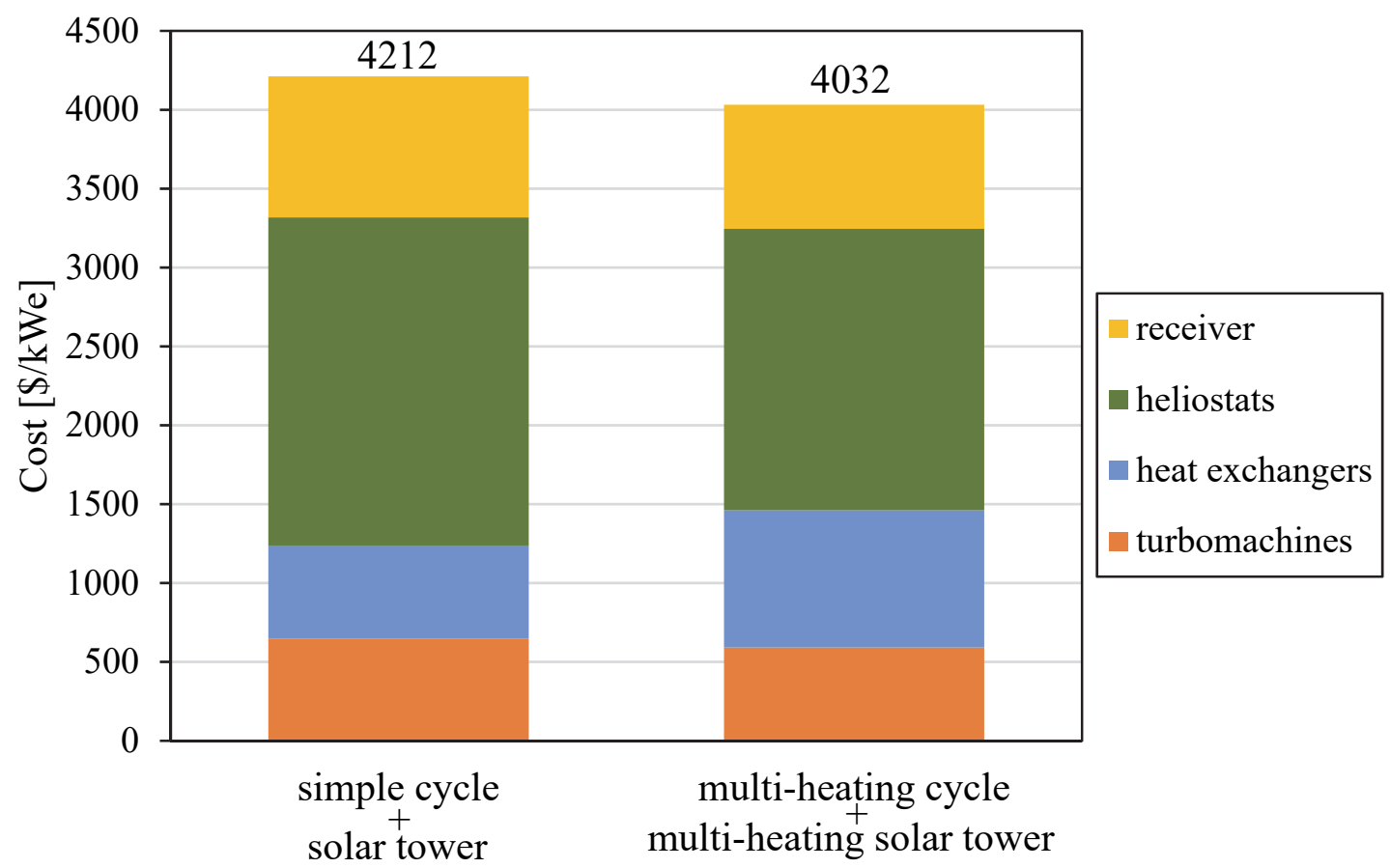

Figure 6.1. Cost comparison between a CSP plant with simple cycle and solar tower and a CSP plant with multi-heating cycle and multi-heating solar tower.

The new system uses the basis of thermal coherence to obtain higher efficiencies with lower material requirements. The new CSP plant layout shows a great potential to reduce the cost of CSP plants by adding multi-heating to the cycle and to the solar tower.

\subsection{Future work}

This work has analysed $\mathrm{CO}_{2}$ as working fluid due its several advantages: stability and relative inertness for the temperature range of interest, non-toxicity, abundance and low cost. However, other fluids able to work in the pericritical region should not be discarded. There are several investigations about alternative fluids able to work in the pericritical region (CocoEnríquez et al., 2017; Guo et al., 2019; Hu et al., 2015). Some of these investigations propose the use of mixtures of fluids with $\mathrm{CO}_{2}$. An interesting option would be to use mixtures of fluids with $\mathrm{CO}_{2}$ to increase its critical temperature, and so bring the compressor closer to the critical temperature in dry-cooled cycles.

The multi-heating cycle is the result of a thermodynamic analysis focused on reducing the temperature of the heat source. Although the first aim was to integrate these cycles in CSP plants, other type of plants or even hybridization between different types of plant could take 
advantage of these cycles too. For example, nuclear power could supply the heat at high temperature and very simple and cheap solar field the heat at low temperature.

The part of the multi-heating solar tower at low temperature and low concentration ratio must be further analysed. This work presented different options for this part of the solar field. A more detailed technoeconomic analysis should be performed to better judge the potential of these options and, therefore, the potential of multi-heating solar towers in CSP plants. Moreover, the thermal energy storage is not included in the CSP plant configuration as it is out of the purpose of this work. However, a complete technoeconomic analysis should include it. This analysis must differentiate between the cost of the thermal energy storage at low temperature and the storage at high temperature.

The presented techno-economic analysis compares simple and multi-heating cycles because the simple system seems to be one of the best options for CSP plants, according to previous investigations (Ho et al., 2015). However, other investigations achieve lower costs with other types of cycle by adding partial cooling (Neises and Turchi, 2019). Multi-heating cycles could integrate partial cooling. A further comparison between all the existing cycles and multiheating with and without partial cooling could reveal the best option. 


\section{REFERENCES}

Ahn, Y., Bae, S.J., Kim, M., Cho, S.K., Baik, S., Lee, J.I. and Cha, J.E. (2015) 'Review of supercritical $\mathrm{CO} 2$ power cycle technology and current status of research and development', Nuclear Engineering and Technology, 47(6), pp. 647-661.

Akbari, A.D. and Mahmoudi, S.M.S. (2014) 'Thermoeconomic analysis \& optimization of the combined supercritical CO2 (carbon dioxide) recompression Brayton/organic Rankine cycle', Energy, 78, pp. 501-512.

Angelino, G. (1968) 'Carbon Dioxide Condensation Cycles For Power Production', Journal of Engineering for Gas Turbines and Power, 90(3), pp. 287-295.

Angelino, G. (1969) 'Real gas effects in carbon dioxide', ASME publication, , pp. 1-12.

Bae, Y.-Y., Kim, H.-Y. and Kang, D.-J. (2010) 'Forced and mixed convection heat transfer to supercritical $\mathrm{CO} 2$ vertically flowing in a uniformly-heated circular tube', Experimental Thermal and Fluid Science, 34(8) Elsevier, pp. 1295-1308. Available at: 10.1016/j.expthermflusci.2010.06.001 (Accessed: 11 July 2018).

Banuti, D., Raju, M. and Ihme, M. (2018) 'On the characterization of transcritical fluid states', Technical report (Stanford University - Center for Turbulence Research)

Banuti, D., Raju, M., Ma, P.C., Ihme, M. and Hickey, J.-P. (2017) 'Seven questions about supercritical fluids - towards a new fluid state diagram', 55th AIAA Aerospace Sciences Meeting

Bejan, A. (2006) Advanced Engineering Thermodynamics. 3rd edn. Danvers, USA: John Wiley \& Sons. 
Bellan, J. (2006) 'Theory, modeling and analysis of turbulent supercritical mixing', Combustion Science and Technology, 178(1-3) Taylor \& Francis, pp. 253-281.

Binotti, M., Astolfi, M., Campanari, S., Manzolini, G. and Silva, P. (2017) 'Preliminary assessment of sCO2 cycles for power generation in CSP solar tower plants', Applied Energy, 204 Elsevier, pp. 1007-1017.

Bryant, J.C., Saari, H. and Zanganeh, K. (2011) 'An Analysis and Comparison of the Simple and Recompression Supercritical CO2 Cycles', Supercritical CO2 Power Cycle Symposium

Cabeza, L.F., de Gracia, A., Fernández, A.I. and Farid, M.M. (2017) 'Supercritical CO2 as heat transfer fluid: A review', Applied Thermal Engineering, 125, pp. 799-810.

Candel, S., Juniper, M., Singla, G., Scouflaire, P. and Rolon, C. (2006) 'Structure and dynamics of cryogenic flames at supercritical pressure', Combustion Science and Technology, 178(1-3) Taylor \& Francis, pp. 161-192.

Carlson, M.D., Middleton, B.M. and Ho, C.K. (2017) 'Cycles Using Component Cost Models Baselined With Vendor Data', Proceedings of the ASME 2017 Power and Energy Conference, , pp. 1-7.

Chen, M., Kim, I.H., Sun, X., Christensen, R., Bartel, N., Utgikar, V. and Sabharwall, P. (2013) 'Design of printed circuit heat exchangers for very high temperature reactors', Transactions of the American Nuclear Society., Vol.108, pp. 1090-1093.

Cheng, X. and Liu, X.J. (2017) 'Research Challenges of Heat Transfer to Supercritical Fluids', Journal of Nuclear Engineering and Radiation Science, 4(1), p. 011003.

Coco-Enríquez, L., Muñoz-Antón, J., Martínez-Val, J.M. and Enriquez, L. (2017) 'New text comparison between $\mathrm{CO} 2$ and other supercritical working fluids (ethane, $\mathrm{Xe}, \mathrm{CH} 4$ and N2) in line- focusing solar power plants coupled to supercritical Brayton power cycles', International Journal of Hydrogen Energy, 42(28) Pergamon, pp. 17611-17631.

Crespi, F., Gavagnin, G., Sánchez, D. and Martínez, G.S. (2017) 'Supercritical carbon dioxide cycles for power generation: A review', Applied Energy, 195 Elsevier, pp. 152-183.

Dostal, V. (2004) A Supercritical Carbon Dioxide Cycle for Next Generation Nuclear Reactors. PhD Thesis. Massachusetts Institute of Technology.

Dunham, M.T. and Iverson, B.D. (2014) 'High-efficiency thermodynamic power cycles for concentrated solar power systems', Renewable and Sustainable Energy Reviews, 30, pp. 758770. 
Dyreby, J. (2014) Modeling the Supercritical Carbon Dioxide Brayton Cycle with Recompression. PhD Thesis. The University of Wisconsin, Madison.

Dyreby, J.J., Klein, S.A., Nellis, G. and Reindl, D.T. (2014) 'Development of a Flexible Modeling Tool for Predicting Optimal Off-Design Performance of Simple and Recompression Brayton Cycles', 4th International Symposium - Supercritical CO2 Power Cycles

EnergyPlus (2018) Weather Data Base. Available at: https://energyplus.net/weather (Accessed: 8 May 2018).

Feher, E.G. (1968) 'The Supercritical Thermodynamic Power Cycle', Energy Conversion, 8, pp. 85-90.

Forristall, R. (2003) 'Heat transfer analysis and modeling of a parabolic trough solar receiver implemented in engineering equation solver', NREL/TP-550-34169

Gezelius, K. (2004) Design of Compact Intermediate Heat Exchangers for Gas Cooled Fast Reactors. Master Thesis. Massachusetts Institute of Technology.

Gnielinski, V. (1976) 'New equations for heat and mass transfer in turbulent pipe and channel flow', Int. Chem. Eng, 16(2), pp. 359-368.

González-Portillo, L.F., Muñoz-Antón, J. and Martínez-Val, J.M. (2017a) 'Comparing the cost of storage in photovoltaics and concentrating solar power: batteries vs thermal energy storages', $10^{\circ}$ Congreso Nacional Ingeniería Termodinámica.

González-Portillo, L.F., Muñoz-Antón, J. and Martínez-Val, J.M. (2017b) 'An analytical optimization of thermal energy storage for electricity cost reduction in solar thermal electric plants', Applied Energy, 185, pp. 531-546.

González-Portillo, L.F., Muñoz-Antón, J. and Martínez-Val, J.M. (2019) 'Thermodynamic mapping of power cycles working around the critical point', Energy Conversion and Management, 192, pp. 359-373.

Guo, J., Li, M., Xu, J., Yan, J. and Wang, K. (2019) 'Thermodynamic performance analysis of different supercritical Brayton cycles using CO2-based binary mixtures in the molten salt solar power tower systems', Energy, 173, pp. 785-798.

Ho, C.K., Carlson, M., Garg, P. and Kumar, P. (2015) 'Cost and Performance Tradeoffs of Alternative Solar-driven S-CO2 Brayton Cycle Configurations', Proceedings of the ASME 2015 Power and Energy Conversion Conference, , pp. 1-10.

Ho, C.K. and Iverson, B.D. (2014) 'Review of high-temperature central receiver designs for 
concentrating solar power', Renewable and Sustainable Energy Reviews, 29, pp. 835-846.

Hu, L., Chen, D., Huang, Y., Li, L., Cao, Y., Yuan, D., Wang, J. and Pan, L. (2015) 'Investigation on the performance of the supercritical Brayton cycle with CO2-based binary mixture as working fl uid for an energy transportation system of a nuclear reactor', Energy, 89, pp. 874-886.

Incropera, F.P., DeWitt, D.P., Bergman, T.L. and Lavine, A.S. (2007) Fundamentals of Heat and Mass Transfer. 6th edn. John Wiley \& Sons.

Invernizzi, C.M. (2017) 'Prospects of Mixtures as Working Fluids in Real-Gas Brayton Cycles', Energies, 10, pp. 1-15.

IRENA (2012) Renewable energy technologies: cost analysis series - Concentrating Solar Power (Power Sector) - Volume 1 Issue 2/5.

Jackson, J.D. (1997) 'Supercritical heat transfer', in A-to-Z Guide to Thermodynamics, Heat and Mass Transfer, and Fluids Engineering. Begellhouse.

Khivsara, S.D., Das, R.N., Thyagaraj, T.L., Dhar, S., Srinivasan, V. and Dutta, P. (2014) 'Development of a Ceramic Pressurized Volumetric Solar Receiver for Supercritical CO2 Brayton Cycle', 12th International Conference on Fuel Cell Science, Engineering and Technology. ASME.

Kim, Y.M., Kim, C.G. and Favrat, D. (2012) 'Transcritical or supercritical CO2 cycles using both low- and high-temperature heat sources', Energy, 43(1), pp. 402-415.

Klein, S. (1992) Engineering Equation Solver (EES) 10.494. F-chart software,

Lei, X., Li, H., Zhang, W., Dinh, N.T., Guo, Y. and Yu, S. (2017) 'Experimental study on the difference of heat transfer characteristics between vertical and horizontal flows of supercritical pressure water', Applied Thermal Engineering, 113, pp. 609-620.

Ma, Y., Liu, Z. and Tian, H. (2013) 'A review of transcritical carbon dioxide heat pump and refrigeration cycles', Energy, 55, pp. 156-172.

Mehos, M., Turchi, C., Vidal, J., Wagner, M., Ma, Z., Ho, C., Kolb, W., Andraka, C. and Kruizenga, A. (2017) 'Concentrating Solar Power Gen3 Demonstration Roadmap', NREL/TP5500-67464

Moisseytsev, A. and Sienicki, J.J. (2014) 'Investigation of a dry air cooling option for an sCO2 cycle', The 4th International Symposium - Supercritical CO2 Power Cycles, 1, pp. 1-21. 
Montes, M.J., Abánades, A., Martínez-Val, J.M. and Valdés, M. (2009) 'Solar multiple optimization for a solar-only thermal power plant, using oil as heat transfer fluid in the parabolic trough collectors', Solar Energy, 83(12), pp. 2165-2176.

Müller, A., Kranzl, L., Tuominen, P., Boelman, E., Molinari, M. and Entrop, A.G. (2011) 'Estimating exergy prices for energy carriers in heating systems: Country analyses of exergy substitution with capital expenditures', Energy and Buildings, 43(12), pp. 3609-3617.

Muñoz, J., Abánades, A. and Martínez-Val, J.M. (2009) 'A conceptual design of solar boiler', Solar Energy, 83(9), pp. 1713-1722.

Muñoz, J., Martinez-Val, J.M. and Ramos, A. (2011) 'Thermal regimes in solar-thermal linear collectors', Solar Energy, 85(5), pp. 857-870.

Neises, T. and Turchi, C. (2014) 'A Comparison of Supercritical Carbon Dioxide Power Cycle Configurations with an Emphasis on CSP Applications', Energy Procedia, 49, pp. 1187-1196.

Neises, T. and Turchi, C. (2019) 'Supercritical carbon dioxide power cycle design and configuration optimization to minimize levelized cost of energy of molten salt power towers operating at $650^{\circ} \mathrm{C}^{\prime}$, Solar Energy, 181, pp. 27-36.

Neises, T.W., Wagner, M.J. and Gray, A.K. (2014) 'Structural Design Considerations for Tubular Power Tower Receivers Operating at $650^{\circ} \mathrm{C}^{\prime}, N R E L / C P-5500-61848$, , pp. 1-7.

NREL (2017) SAM (System Advisor Model) version 2017.9.5 2017.9.5.

Padilla, R.V., Soo Too, Y.C., Benito, R. and Stein, W. (2015a) 'Exergetic analysis of supercritical CO2 Brayton cycles integrated with solar central receivers', Applied Energy, 148, pp. 348-365.

Padilla, R.V., Too, Y.C.S., Beath, A., McNaughton, R. and Stein, W. (2015b) 'Effect of Pressure Drop and Reheating on Thermal and Exergetic Performance of Supercritical Carbon Dioxide Brayton Cycles Integrated With a Solar Central Receiver', Journal of Solar Energy Engineering, 137(5), p. 051012.

Pérez-Pichel, G.D., Linares, J.I., Herranz, L.E. and Moratilla, B.Y. (2012) 'Thermal analysis of supercritical CO2 power cycles: Assessment of their suitability to the forthcoming sodium fast reactors', Nuclear Engineering and Design, 250 North-Holland, pp. 23-34.

Pfleger, N., Bauer, T., Martin, C., Eck, M. and Wörner, A. (2015) 'Thermal energy storage overview and specific insight into nitrate salts for sensible and latent heat storage', Beilstein 
journal of nanotechnology, 6 Beilstein-Institut, pp. 1487-1497.

Pioro, I., Mahdi, M. and Popov, R. (2017) 'Application of supercritical pressures in power engineering', in Advanced Applications of Supercritical Fluids in Energy Systems. , pp. 404457.

Pioro, I. and Mokry, S. (2011) 'Heat Transfer To Fluids At Supercritical Pressure', in Belmiloudi, A. (ed.) Theoretical Analysis, Experimental Investigations and Industrial Systems. IntechOpen, pp. 481-504.

Pioro, I.L. (2016) 'Heat transfer and pressure drop in forced convection to fluids at supercritical pressures', in Handbook of Generation IV Nuclear Reactors. Elsevier Ltd, pp. $795-823$.

Pioro, I.L. and Zvorykin, C.O. (2016) 'Thermophysical properties of fluids at subcritical and critical/supercritical conditions', in Handbook of Generation IV Nuclear Reactors. Elsevier, pp. 771-794.

Pye, J., Zheng, M., Asselineau, C.-A. and Coventry, J. (2014) 'An exergy analysis of tubular solar-thermal receivers with different working fluids', International Conference on Concentrating Solar Power and Chemical Energy Systems, SolarPACES 2014.

Rovira, A., Muñoz-Antón, J., Montes, M.J. and Martínez-Val, J.M. (2013) 'Optimization of Brayton cycles for low-to-moderate grade thermal energy sources', Energy, 55, pp. 403-416.

Sait, H.H., Martinez-Val, J.M., Abbas, R. and Munoz-Anton, J. (2015) 'Fresnel-based modular solar fields for performance/cost optimization in solar thermal power plants: A comparison with parabolic trough collectors', Applied Energy, 141, pp. 175-189.

Sánchez Villafana, E.D. and Vargas Machuca Bueno, J.P. (2019) 'Thermoeconomic and environmental analysis and optimization of the supercritical $\mathrm{CO} 2$ cycle integration in a simple cycle power plant', Applied Thermal Engineering, 152, pp. 1-12.

Sarkar, J. (2015) 'Review and future trends of supercritical CO2 Rankine cycle for low-grade heat conversion', Renewable and Sustainable Energy Reviews, 48, pp. 434-451.

Sarkar, J. (2009) 'Second law analysis of supercritical CO2 recompression Brayton cycle', Energy, 34(9), pp. 1172-1178.

Schleicher, R., Raffray, A.R. and Wong, C.P. (2001) 'An Assessment of the Brayton Cycle for High Performance Power Plants', Fusion Technology, 39(2)

Sciortino, F., Poole, P., Essmann, U. and Stanley, H. (1997) 'Line of compressibility maxima 
in the phase diagram of supercooled water', Physical Review E, 55

Swinbank, W.C. (1963) 'Long-wave radiation from clear skies', Quarterly Journal of the Royal Meteorological Society, 89(381) John Wiley \& Sons, Ltd, pp. 339-348.

Syblik, J., Vesely, L., Entler, S., Stepanek, J. and Dostal, V. (2019) 'Analysis of supercritical $\mathrm{CO} 2$ Brayton power cycles in nuclear and fusion energy', Fusion Engineering and Design, In Press

Teng, L. and Xuan, Y. (2019) 'A Novel Solar Receiver for Supercritical CO2 Brayton Cycle', Energy Procedia, 158, pp. 339-344.

Tucker, S.C. (1999) 'Solvent Density Inhomogeneities in Supercritical Fluids', Chemical Reviews, 99(2) American Chemical Society, pp. 391-418.

Turchi, C.S., Ma, Z., Neises, T. and Wagner, M. (2012) 'Thermodynamic Study of Advanced Supercritical Carbon Dioxide Power Cycles for High Performance Concentrating Solar Power Systems', ASME 2012 6th International Conference on Energy Sustainability.

Wagner, M.J. and Wendelin, T. (2018) 'SolarPILOT: A power tower solar field layout and characterization tool', Solar Energy, 171, pp. 185-196.

Wang, K., He, Y.-L. and Zhu, H.-H. (2017) 'Integration between supercritical CO2 Brayton cycles and molten salt solar power towers: A review and a comprehensive comparison of different cycle layouts', Applied Energy, 195, pp. 819-836.

Younglove, B.A. (1982) 'Thermophysical Properties of Fluids. I. Argon, Ethylene, Parahydrogen, Nitrogen, Nitrogen Trifluoride, and Oxygen', Journal of Physical and Chemical Reference Data, 14(2), pp. 619-630. 


\section{APPENDICES}




\section{APPENDIX A - FLUID CANDIDATES FOR PERICRITICAL CYCLES}

\begin{tabular}{|c|c|c|}
\hline Fluid & $\mathbf{P}_{\mathrm{cr}}[\mathrm{MPa}]$ & $\mathbf{T}_{\mathrm{cr}}[\mathbf{K}]$ \\
\hline Ethylene & 5.04 & 284.35 \\
\hline R508B & 3.93 & 289.14 \\
\hline Xenon & 5.84 & 291.74 \\
\hline R116 & 3.04 & 295.03 \\
\hline $\mathrm{R} 23$ & 4.83 & 301.28 \\
\hline $\mathrm{R} 13$ & 3.87 & 304.01 \\
\hline $\mathrm{CO} 2$ & 7.38 & 306.13 \\
\hline Ethane & 4.87 & 307.32 \\
\hline $\mathrm{R} 41$ & 5.90 & 319.28 \\
\hline SF6 & 3.76 & 320.72 \\
\hline R125 & 3.62 & 341.17 \\
\hline R507A & 3.71 & 345.76 \\
\hline R410A & 4.90 & 346.49 \\
\hline R218 & 2.64 & 347.02 \\
\hline R404A & 3.74 & 347.27 \\
\hline R143A & 3.76 & 347.85 \\
\hline
\end{tabular}




\section{APPENDIX B - CORRELATIONS USED IN THE HEAT EXCHANGER DESIGN}

\section{Heat transfer model}

The correlation for the turbulent flow regime $(\operatorname{Re}>2300)$ used is the Gnielinski correlation:

$$
N u=\frac{\frac{f_{c}}{8}(\operatorname{Re}-1000) \operatorname{Pr}}{1+12.7\left(\operatorname{Pr}^{2 / 3}-1\right) \sqrt{\frac{f_{c}}{8}}}
$$

where $N u$ is the Nusselt number, $R e$ is the Reynolds number, $P r$ is the Prandtl number and $f_{c}$ the Moody friction factor defined as:

$$
f_{c}=\left(\frac{1}{1.8 \log _{10} R e-1.5}\right)^{2}
$$

The Reynolds number is defined as:

$$
R e=\frac{v d_{h}}{v}
$$

where $v$ is the fluid velocity, $d_{h}$ the hydraulic diameter and $v$ the fluid kinematic viscosity. The hydraulic diameter for the semi-circular channel is defined as:

$$
d_{h}=\frac{4 \pi d_{c}^{2}}{8\left(\pi \frac{d_{c}}{2}+d_{c}\right)}
$$

where $d_{c}$ is the semi-circular channel diameter.

For laminar flow $(R e<2300)$ the Nusselt number is set to $\mathrm{Nu}=4.089$.

Once the Nusselt number is known the heat transfer coefficient htc can be calculated as:

$$
h t c=\frac{N u \cdot k}{d_{h}}
$$

where $k$ is the fluid conductivity. 


\section{Pressure drop model}

Different correlations are used to calculate the friction factor $f$. The relative roughness $\Delta$ (ratio of surface roughness and tube diameter) and the Reynolds number Re determine which correlation must be used in each case. The value of the Reynolds number divides the behavior of the friction factor into five regions, separated by the Reynolds numbers $R e_{0}, R e_{1}, R e_{2}$ and $\operatorname{Re}_{3}$.

If the relative roughness is $\Delta>0.007$ then the values of $R e_{0}$ and $R e_{1}$ are:

$$
\begin{gathered}
R e_{0}=754 e^{\frac{0.0065}{\Delta}} \\
R e_{1}=\frac{1160}{\Delta^{0.11}}
\end{gathered}
$$

If the relative roughness is $\Delta<0.007$ then the values of $R e_{0}$ and $R e_{1}$ are $R e=2000$.

Regardeless of the relative roughness, the values of $R e_{2}$ and $R e_{3}$ are:

$$
\begin{aligned}
R e_{2} & =\frac{2090}{\Delta^{0.0635}} \\
R e_{3} & =\frac{441.19}{\Delta^{1.1772}}
\end{aligned}
$$

For laminar flow, i.e., for Reynolds number below $R e_{0}$, the friction factor is:

$$
f=\frac{64}{R e}
$$

For Reynolds number above $R e_{0}$ but below $R e_{1}$, the friction factor is:

$$
f=4.4 R e^{-0.595} e^{\frac{-0.00275}{\Delta}}
$$

For Reynolds number above $R e_{1}$ but below $R e_{2}$, the friction factor is:

$$
f=\left(f_{2}-f^{*}\right) e^{-\left(0.0017\left(R e_{2}-R e\right)\right)^{2}}+f^{*}
$$

where $f^{*}$ is .032 when $\Delta<0.007$ and

$$
f^{*}=0.0733-\frac{0.0109}{\Delta^{0.286}}
$$

when $\Delta>0.007$.

For Reynolds number above $\operatorname{Re}_{2}$ but below $\operatorname{Re}_{3}$ the friction factor is:

$$
f=\left(2 \log _{10}\left(\frac{2.51}{R e \sqrt{f}}+\frac{\Delta}{3.7}\right)\right)^{-2}
$$


And for Reynolds number above $R e_{3}$ the same expression can be used by substituting $R e$ by $\mathrm{Re}_{3}$. 


\section{APPENDiX C - SPECIFICATIONS SHEET OF PRINTEd CiRCUIT HEAT EXCHANGER}

Specification sheet of a Printed Circuit Heat Exchanger from Heatric (Dostal, 2004):

\begin{tabular}{|c|c|}
\hline Unit weight range & $\begin{array}{l}1 \mathrm{~kg} \text { to } 60 \text { tonnes as a single unit } \\
\text { However larger modular assemblies are possible }\end{array}$ \\
\hline Maximum design pressure & Current maximum design pressure 650 bar (9500 psi) \\
\hline Design temperature range & Currently from $2 \mathrm{~K}$ to $900^{\circ} \mathrm{C}\left(4 \mathrm{R}\right.$ to $\left.1650^{\circ} \mathrm{F}\right)$ \\
\hline Maximum nozzle size & $900 \mathrm{~mm}$ \\
\hline Maximum surface area & $10,000 \mathrm{~m}^{2}\left(108,000 \mathrm{ft}^{2}\right)$ per PCHE \\
\hline Typical area/unit volume & $\begin{array}{l}1300 \mathrm{~m}^{2} / \mathrm{m}^{3} \text { at } 100 \text { bar }\left(400 \mathrm{ft}^{2} / \mathrm{ft}^{3} \text { at } 1450 \mathrm{psi}\right) \\
650 \mathrm{~m}^{2} / \mathrm{m}^{3} \text { at } 500 \text { bar }\left(200 \mathrm{ft}^{2} / \mathrm{ft}^{3} \text { at } 7250 \mathrm{psi}\right)\end{array}$ \\
\hline $\begin{array}{l}\text { Minimum temperature } \\
\text { approach }\end{array}$ & $1^{\circ} \mathrm{C}$ (typically $3-5^{\circ} \mathrm{C}$ ) $2^{\circ} \mathrm{F}$ (typically $5-10^{\circ} \mathrm{F}$ ) \\
\hline Heat exchanger effectiveness & up to $98 \%$ \\
\hline $\begin{array}{l}\text { Typical overall heat transfer } \\
\text { coefficients }\end{array}$ & $\begin{array}{l}\text { LP gas cooler } 500-1,000 \mathrm{~W} / \mathrm{m}^{2} \mathrm{~K}\left(90-180 \mathrm{Btu} / \mathrm{hrft}^{2}{ }^{\circ} \mathrm{F}\right) \\
\mathrm{HP} \text { gas cooler } 1,000-4,000 \mathrm{~W} / \mathrm{m}^{2} \mathrm{~K}\left(180-700 \mathrm{Btu} / \mathrm{hrft}^{2 \circ} \mathrm{F}\right) \\
\text { Water/water } 7,000-10,000 \mathrm{~W} / \mathrm{m}^{2} \mathrm{~K}\left(1230-1750 \mathrm{Btu} / \mathrm{hrft}^{2} \mathrm{~F}\right)\end{array}$ \\
\hline Plate thickness & $0.5 \mathrm{~mm}^{(1)}$ to $5.0 \mathrm{~mm}$ \\
\hline Passage width & $0.5 \mathrm{~mm}$ to $5.0 \mathrm{~mm}^{(2)}$ \\
\hline Typical Reynolds number range & $\begin{array}{l}\text { Gases: } 1,000-100,000 \\
\text { Liquids: } 10-5,000\end{array}$ \\
\hline
\end{tabular}

